\title{
Generación y procesamiento de señales ópticas coherentes mediante sistemas de fibra óptica.
}

Tesis doctoral presentada por

\author{
Emanuel Paulucci \\ ante la \\ Facultad de Ingeniería de la \\ Universidad Nacional de La Plata \\ para acceder al grado académico de \\ DOCTOR EN INGENIERÍA
}

Dirección de la tesis:

Director: Dr. Ricardo Duchowicz.

Co-Director: Dr. Enrique E. Sicre.

Lugar de realización:

Centro de Investigaciones Ópticas CIOp (CONICET - CIC)

La Plata, Lunes 20 de Mayo de 2013. 
Dedicado a mis padres Alejandro y Lucía, mi hermano Ezequiel y mis amigos! 


\section{Agradecimientos}

Quiero expresar mi agradecimiento a mis directores, al Dr. Ricardo Duchowicz y Dr. Enrique Sicre por su permanente colaboración, a mis compañeros de trabajo el Dr. Pablo Costanzo Caso y Dr. Laureano Bulus Rossini por su ayuda incondicional en todo momento, y en particular quiero dar las gracias a la Dra. Nélida Russo por su colaboración en las tareas de laboratorio. Agradezco a los directivos y demás integrantes del Centro de Investigaciones Ópticas (CIOp) que me brindaron las condiciones necesarias para que este trabajo sea posible, al Dr. Agustín Roncagliolo y el Ing. Adrián Carlotto del Laboratorio de Electrónica Industrial Control e Instrumentación (LEICI) de la Facultad de Ingeniería de la Universidad Nacional de La Plata (UNLP), por su apoyo en las tareas de docencia realizadas en la cátedra de Comunicaciones, y al Dr. Carlos Muravchik en la cátedra de Señales y Sistemas. Quiero agradecer a las instituciones que financiaron mi trabajo como becario de posgrado, al Consejo Nacional de Investigaciones Científicas de la Provincia de Buenos Aires (CIC), la Agencia Nacional de Promoción Científica y Tecnológica (ANPCyT) a través de la Fundación Ciencias Exactas, la Comisión de Investigaciones Científicas y Técnicas (CONICET), y en especial al Director Ejecutivo del Departamento de Electrotecnia de la Facultad de Ingeniería de la UNLP Ing. José Roberto Vignoni. Finalmente, agradezco enormemente la colaboración como revisores y miembros del jurado de este trabajo de tesis doctoral al Dr. Diego Grosz (ITBA) y Dr. Héctor Ranea Sandoval (IFAS). 


\section{Acrónimos}

APM suma de pulsos por enganche de modos

ASE emisión espontánea amplificada

CD dispersión cromática

CDM multiplexado por división de código

CW onda continua

BER tasa de error de bit

DFB realimentación distribuida

EDFL láser de FO dopada con $\mathrm{Er}^{3+}$

EMI interferencia electromagnética

EO electro-óptica

FBG red de Bragg grabada en fibra

FFT transformada rápida de Fourier

FO fibra óptica

FOV campo de visión

FPI interferómetro Fabry-Perot 
FSR rango espectral libre

FIR infrarrojo lejano

FWHM ancho medido a mitad del máximo

FWM mezcla de cuatro ondas

ISI interferencia intersímbolo

LASER amplificación de la luz mediante la emisión estimulada de radiación

MASER amplificación de microondas mediante la emisión estimulada de radiación

MI interferómetro Michelson

MZI interferómetro Mach-Zehnder

NLSE ecuación no-lineal de Schrödinger

NIR infrarroja cercana

OE optoeléctrica

PAA conjunto de antenas en fase

PMD dispersión por modo de polarización

PSD densidad espectral de potencia

SBS esparcimiento Brillouin estimulado

SIR infrarrojo de onda corta

SNR relación señal ruido

SPM automodulación de fase

SRS esparcimiento Raman estimulado 
SSM método de pasos divididos

SVEA aproximación de variación suave de la envolvente

TDM multiplexación por división de tiempo

TMM método de la matriz de transferencia

TOD dispersión de tercer orden

UV ultravioleta

WDF función de distribución de Wigner

WDM multiplexación por división de longitud de onda

XPM modulación cruzada de la fase 


\section{Glosario}

chirp en el ámbito de las comunicaciones, se entiende como una pequeña variación lineal o no-lineal de la fase de una entidad física o matemática.

fotón en física moderna, es la partícula elemental responsable de las manifestaciones cuánticas del fenómeno electromagnético, en particular de la luz.

fotónico proceso vinculado a la interacción luz-materia.

intensidad módulo al cuadrado de un campo eléctrico.

jitter desviación no deseada del período de una señal asumida en la electrónica y las telecomunicaciones, a menudo en relación con una fuente de reloj de referencia.

núcleo parte interna de una fibra óptica de geometría cilíndrica, con un índice de refracción $n_{\text {core }}$, por donde se propaga casi toda la luz.

reflectividad módulo al cuadrado del coeficiente de reflexión.

revestimiento parte externa de una fibra óptica que cubre al núcleo, cuyo índice de refracción es $n_{\text {cladding }}<n_{\text {core }}$, lo que permite confinar la luz en el núcleo mediante la ley de Snell.

solitón onda que mantiene sus propiedades tiempo-frecuencia inalteradas, a medida que se propaga por un medio de transmisión no-lineal y dispersivo como la FO.

transmitancia módulo al cuadrado del coeficiente de transmisión. 


\section{Índice de figuras}

2.1. Atenuación de una FO estándar (SMF28 Corning) en función de la longitud de onda. . . . . . . . . . . . . . . . . .

2.2. Dispersión de una FO estándar (SMF28 Corning) en función de la longitud de onda, expresada en nanometros. . . . . . . . . . . . . . . .

2.3. Propagación de un pulso gaussiano real de ancho $T_{0}$ sobre una FO de longitud $2 L_{D}$. El efecto de la dispersión bajo el régimen anómalo produce un ensanchamiento temporal del pulso a medida que se propaga por la FO, mientras que el espectro de dicho pulso resulta inalterado. (a) Evolución temporal en función de la longitud de propagación. (b) Densidad espectral de energía del pulso propagado. . . . . . . . . . . . . . . . .

2.4. Evolución de la intensidad y el ancho temporal del pulso gaussiano, para una longitud de propagación $z=2 L_{D}$. La intensidad es representada en línea de trazos. . . . . . . . . . . . . . . . . . . . . .

2.5. (a) Evolución del pulso propagado con SPM en el dominio temporal. (b) Densidad espectral de energía del pulso propagado. . . . . . . . . . . . .

2.6. Representación del chirp inducido en el espectro $\left(\delta \omega T_{0}\right)$ por medio del proceso de la SPM. El eje de ordenadas describe la ubicación temporal de la distorsión en función de la longitud de propagación. . . . . . . . . . . . 
2.7. Propagación de un pulso gaussiano para $N \approx 1$. (a) Evolución del pulso en el dominio temporal. (b) Espectro del pulso propagado. (c) Intensidad (línea de trazos) y ancho temporal del pulso en función de la longitud de propagación. (d) Densidad espectral (línea de trazos) y ancho de banda. . .

2.8. Propagación de un pulso secante hiperbólica para $N \approx 1$. (a) Evolución del pulso en el dominio temporal. (b) Espectro del pulso propagado. (c) Intensidad (línea de trazos) y ancho temporal de pulso en función de la longitud de propagación. (d) Densidad espectral (línea de trazos) y ancho de banda.

3.1. (a) Reflectividad de una FBG uniforme para $\kappa L=2$ y 8. (b) Fase del coeficiente de reflexión en unidades arbitrarias. . . . . . . . . . . . .

3.2. (a) Error porcentual del valor de la reflectividad empleando el TMM. (b) Error en la fase del coeficiente de reflexión. . . . . . . . . . . . . . . . . .

3.3. Esquema de una FBG de período no-uniforme o con chirp lineal, también denominadas LCFBG.

3.4. Reflectividad y retardo de grupo de una LCFBG de largo $L \approx 12 \mathrm{~cm}$, ancho de banda $\Delta \lambda_{L C F B G}=0,8 \mathrm{~nm}$, reflectividad $|\rho|^{2}=0,99$ y pendiente del retardo de grupo $\Phi_{20}^{r}=312,5 \mathrm{ps} / \mathrm{nm}$. . . . . . . . . . . . .

3.5. Esquema de un filtro Fabry-Perot con dos espejos de reflectividad $\left|\rho_{\text {mirror }}\right|^{2}$ separados una distancia $d \ldots \ldots \ldots \ldots \ldots$

3.6. (a) Transmitancia del FPI. (b) Retardo de grupo del coeficiente de transmisión. 44

3.7. Esquema del filtro DFB basado en una FBG uniforme y una discontinuidad del período de grabado ubicada en el centro de la misma. . . . . . . . . . .

3.8. (a) Transmitancia de dos DFBs basados en FBGs. (b) Fase del coeficiente de transmisión de los filtros. . . . . . . . . . . . . . . . 
3.9. Características de transmisión de tres DFBs. Un cambio en la longitud de la discontinuidad permite modificar la posición espectral del pico de transmitancia. (a) Módulo al cuadrado del coeficiente de transmisión. (b) Retardo de grupo. . . . . . . . . . . . . . . . . . .

3.10. Característica de la transmisión de dos FPIs implementados con una estructura del tipo DFB basada en una FBG. (a) Transmitancia de dos FPIs diseñados con una discontinuidad de longitud $d \approx 2,5 \mathrm{~mm}$. (b) Retardo de grupo correspondiente a los coeficientes de transmisión de dichos FPIs. . .

3.11. Comparación entre la respuesta de un FPI ideal y uno formado con FBGs. Las respuestas presentan valores de frecuencia similares para una longitud de cavidad $L_{e f f} \ldots \ldots \ldots \ldots \ldots \ldots \ldots$

3.12. Esquema de un láser de FO que opera en régimen $Q$-switch basado en una cavidad del tipo FPI con FBGs y un medio de ganancia provisto por una FO dopada con $\mathrm{Er}^{3+}$. Una de las FBGs esta sujeta mecánicamente a un actuador piezoeléctrico PZT-5H de forma radial para modular las pérdidas de la cavidad. . . . . . . . . . . . . . . . . . . . . . .

3.13. Respuesta espectral de la cavidad tipo FPI formada por dos FBGs y una FO dopada con $\mathrm{Er}^{3+}$. (a) La reflectividad de la cavidad tipo FPI es pequeña cuando la excitación del PZT es nula (no hay modulación externa), lo que induce una pérdida mayor, anulando la salida del láser. (b) Cambio de la respuesta espectral de la cavidad para diferentes valores de modulación. Se encuentran valores de reflectividad mayores para excitaciones negativas (tensión negativa sobre el PZT) . . . . . . . . . . . . .

3.14. Esquema de funcionamiento del régimen Q-switch del láser de FO basado en una cavidad tipo FPI basada en FBGs. Suponiendo una condición de estado inicial excitado, al producirse una conmutación de las pérdidas se produce una inversión de la población del medio activo. La emisión láser ocurre dentro de un intervalo de tiempo en el cual la ganancia es mayor a las pérdidas. . . . . . . . . . . . . . . . . . . 
3.15. Intensidad de salida y excitación aplicada al PZT del láser esquematizado en la Fig. 3.12. (a) Excitación sinusoidal del PZT a la frecuencia de resonancia radial de $18 \mathrm{KHz}$. (b) ídem para una onda cuadrada. . . . . . . . . . . . . .

3.16. (a) Esquema de un acoplador de FO de cuatro puertos, donde $L_{c}$ es la longitud de interacción que define el coeficiente de acoplamiento del dispositivo. (b) Intensidad de salida de los puertos 3 y 4 del acoplador de FO en función del parámetro $\kappa L_{c} \ldots \ldots \ldots \ldots$. . . . . . . . . . . . . . . 5

3.17. Esquema de un MI implementado con un acoplador de FO y dos FBGs. . .

3.18. (a) Intensidad de los campos eléctricos contrapropagantes que viajan hacia los puertos 1 y 2 (salida del interferómetro). (b) Intensidad de los campos eléctricos propagados por las FBGs. Ambos resultados son mostrados en

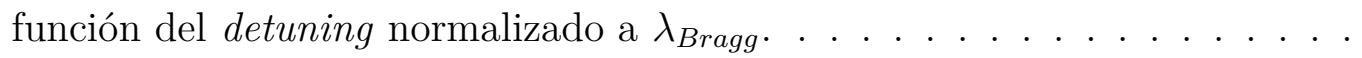

3.19. Modulación de la intensidad transmitida por el puerto 1 (a) y 2 (b) en función de la diferencia de camino normalizada a la longitud de onda de Bragg. . . . . . . . . . . . . . . . . . .

3.20. Esquema de medición del filtro MI implementado con dos FBGs. . . . . . .

3.21. (a) Medida de la intensidad sobre el puerto 2 del MI, obtenida por reflexión de las FBGs. (b) Intensidad de los puertos 3 y 4, medidas por transmisión.

3.22. (a) Esquema de un interferómetro de Mach-Zehnder formado por dos acopladores de FO del $50 \%$. (b) Intensidades transmitidas en función del desplazamiento de la fase $\Delta \phi$ (debido a una diferencia de camino óptico entre las ramas del MZI) . . . . . . . . . . . . . . . .

3.23. Esquema de un interferómetro de Mach-Zehnder formado por dos acopladores de FO y dos FBGs.

3.24. (a) Intensidad de los campos eléctricos contrapropagantes que viajan hacia los puertos 11 y 21 (salida del interferómetro por reflexión). (b) Transmitancias de salida del MZI. . . . . . . . . . . . . . . . . 
3.25. Intensidad de los campos eléctricos propagados en función del desajuste de fase $\Delta \theta$. (a) Respuesta del puerto de "subida" del interferómetro en función del desajuste. (b) Transmitancia de salida para diferentes condiciones de

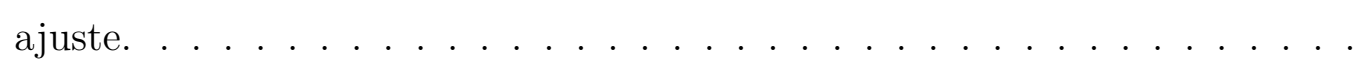

3.26. Esquema de medición del MZI implementado con dos acopladores de FO y dos FBGs. . . . . . . . . . . . . . . . .

3.27. (a) Intensidad reflejada por cada una de las FBGs empleadas en la implementación del MI y MZI (medidas de forma separada). (b) Medida de la intensidad sobre el puerto 2 del MZ, obtenida por reflexión de las FBGs. (c) Señal fotodetectada en los puertos 3 y 4 del MZI para una condición de ajuste adecuada. (d) Intensidad de los puertos 3 y 4 para un desajuste del MZI . . . . . . . . . . . . . . . . .

3.28. Esquema de un phased-array antenna (PAA) para la formación de haces de RF o beamforming. El procesamiento puede ser implementado mediante desplazadores de fase en el dominio analógico, de forma digital, o bien en el domino óptico mediante el diseño de líneas de retardo.

3.29. Línea de retardo ópticas basadas FO estándar y FBG. Se expresa una única línea o rama del sistema de OBF donde se muestran los principales componentes del mismo. . . . . . . . . . . . . . . . . . . . .

3.30. Señales fotodetectadas en la etapa de conversión OE, las cuales son eventualmente amplificadas para excitar el PAA. Se muestra la salida para (a) $\theta_{3}=0^{\circ}$ y (b) $\theta_{2}=45^{\circ} \ldots \ldots \ldots \ldots \ldots \ldots$

3.31. Generación de los haces electromagnéticos mediante un sistema OBF y un PAA formado por 4 antenas isotrópicas. Los diagramas muestran la potencia del campo resultante medida a $300 \mathrm{~m}$ del PAA, para los ángulos de diseño especificados. (a) $\theta_{3}=0^{\circ}$. (b) $\theta_{2}=45^{\circ}$. (b) $\theta_{1}=-45^{\circ}$...........

3.32. (a) Estimación de la relación portadora ruido del sistema OBF. (b) Medida de distorsión de segundo orden y de tercer orden relacionada a los productos de intermodulación mencionados. . . . . . . . . . . . . . . . . . . . 
3.33. Diagrama esquemático de la implementación del dispositivo que permite recuperar la fase y la intensidad de un pulso a partir de dos detecciones de la intensidad del pulso dispersado. La LCFBG presenta un parámetro de dispersión de segundo orden $\Phi_{20}^{r}$. $\mathrm{OC}_{1}$ y $\mathrm{OC}_{2}$ : circuladores ópticos. . . . . .

3.34. Resultados de la recuperación de un pulso de tipo secante hiperbólica de duración $T_{0}=100$ ps y chirp lineal con parámetro $C=6$. (a) y (b) muestran la reflectividad y el retardo de grupo con pendiente $\Phi_{20}^{r}=0,166 \mathrm{ps} / \mathrm{GHz}$ de una LCFBG de $5 \mathrm{~cm}$ de longitud con apodización de tipo coseno elevado y sin apodización, respectivamente. (c) y (d) muestran la intensidad y la fase originales (en línea llena) y recuperadas (en línea a trazos), para los casos especificados en (a) y (b), respectivamente. . . . . . . . . . . .

4.1. Esquema general de una cavidad LASER. . . . . . . . . . . . . . . .

4.2. Diagrama simplificado de los niveles de energía del ion $\operatorname{Er}^{3+}$. La longitud de onda de excitación más importante corresponde a $980 \mathrm{~nm}$, mientras que la ASE se produce para longitudes de onda cercanas a los 1,53 $\mu \mathrm{m}$. (a) La transición ${ }^{4} I_{11 / 2} \rightarrow{ }^{4} I_{13 / 2}$ es no-radiativa. (b) Un sistema de cuatro niveles es también probable, en general para longitudes de onda mayor a 1,55 $\mathrm{mm}$, donde la emisión supera a las pérdidas por absorción. . . . . . . . . . . . .

4.3. Medida de los espectros de absorción y la emisión normalizados del ion $\mathrm{Er}^{+3}$ de una FO dopada (LIEKKI Er30-4). Para $\lambda \approx 1,53 \mu \mathrm{m}$ la pérdida por absorción iguala a la emisión y en estado excitado se produce una ganancia. 99 
4.4. Esquema del láser de FO de dos cavidades acopladas mediante una FBG. La cavidad activa está definida por dos FBGs y una FO dopada con $\operatorname{Er}^{3+}$, mientras que la cavidad auxiliar, acoplada a la anterior por medio de una FBG común a ambas está construida con un tramo de FO estándar cuyo extremo restante es cortado de forma adecuada para obtener un dispositivo de baja reflectividad (reflexión de Fresnel). El sistema opera en régimen APM cuando se verifica la condición de ajuste de la fase, definida por la Ec. 4.8. . . . . . . . . . . . . . . . . . . 106

4.5. Potencia y fase del campo eléctrico resultante de la diagonal secundaria del sistema. El primer término de la Ec. 4.13 aplicado al campo $E_{i 1}$ tiende a ser nulo.

4.6. (a) Potencia del campo eléctrico $E_{i 1}$, solución de láser APM. (b) Fase de $E_{i 1}$ en función del tiempo. . . . . . . . . . . . . . . . . . . . . 116

4.7. Características de las FBGs utilizadas en nuestro modelo numérico. (a) Reflectividad y (b) dispersión en función del detuning normalizado a la longitud de onda de Bragg $\left(\left(\lambda-\lambda_{\text {Bragg }}\right) / \lambda_{\text {Bragg }}\right)$. . . . . . . . . . . . . . . . 118

4.8. Diagrama de flujos del modelo numérico implementado para un láser APM pasivo de dos cavidades acopladas donde $L_{M}=L_{A}(K=1)$. Los campos provenientes de ambas ramas del diagrama deben ser sincronizados adecuadamente en tiempo y espacio para evitar errores sistemáticos en el cálculo de la salida del láser.

4.9. (a) Evolución temporal de la potencia del pulso de entrada para $K=1$. Las características del pulso propagado se mantienen durante más de 1000 roundtrips (equivalente a 20 us aproximadamente), lo que permite establecer una solución estable. (b) Espectro del pulso de entrada y salida. El ensanchamiento espectral se debe al efecto del proceso no-lineal de SPM. . . . . 120

4.10. (a) Fase del pulso en el dominio del tiempo. (b) Fase de la transformada de Fourier del pulso de salida. . . . . . . . . . . . . . . . . . . . . . . 12 
4.11. (a) Evolución temporal de la señal ruidosa de entrada en función del número de roundtrips. (b) Estimación del ancho promedio del pulso de salida. . . . 124

4.12. (a) Autocorrelación del proceso de salida en función de la diferencia de tiempos $\tau$. (b) Estimación de la PSD del proceso calculada mediante los últimos 5 ps de simulación. . . . . . . . . . . . . . . . . . . . . 125

4.13. Simulación de la potencia del campo eléctrico de salida para diferentes valores de $K$. (a) $K=1$ ( $\left.T_{\text {out }}=3,46 \mathrm{~ns}\right)$. (b) $K=2$ ( $\left.T_{\text {out }}=2,26 \mathrm{~ns}\right)$. (c) $K=$ $3\left(T_{\text {out }}=1,74 \mathrm{~ns}\right) .(\mathrm{d}) K=4\left(T_{\text {out }}=1,45 \mathrm{~ns}\right) \ldots \ldots \ldots \ldots$

4.14. Evolución de la energía del pulso simulada en función de la potencia máxima y de la relación de longitudes $K=1$ y $K=2 \ldots \ldots \ldots$. . . . . .

4.15. Estimación de la PSD de la señal de salida para distintos valores de $K$. El incremento en el ancho de banda de la señal de salida se debe principalmente a la disminución del ancho de pulso, cuando la longitud de la cavidad auxiliar $L_{A} \leq L_{M}$. Con "о" se muestran los primeros 50 armónicos correspondientes a cada frecuencia láser de salida. (a) $K=1$ ( $T_{\text {out }}=3,46 \mathrm{~ns}$ ). (b) $K=2$ $\left(T_{\text {out }}=2,26 \mathrm{~ns}\right) .(\mathrm{c}) K=3\left(T_{\text {out }}=1,74 \mathrm{~ns}\right) .(\mathrm{d}) K=4\left(T_{\text {out }}=1,45 \mathrm{~ns}\right)$.

4.16. Esquema de la cavidad principal basada en la reflectividad de las FBGs. 131

4.17. Características del filtro lineal formado por las FBGs que conforman la cavidad principal del láser APM para distintos valores de separación espectral entre las mismas, $\left(f_{\text {Bragg }_{2}}-f_{\text {Bragg }_{1}}\right) / f_{\text {Bragg }_{1}}$. (a) Dispersión de la cavidad, resultante del producto de los coeficientes de reflexión de las FBGs que la componen. (b) Producto de las reflectividades de ambas FBGs en función de la separación espectral relativa entre las mismas. . . . . . . . . . . . .

4.18. Característica temporal del coeficiente de reflexión $\Gamma_{a}(t)$, correspondiente al subsistema formado por la cavidad auxiliar. (a) Reflectividad del mismo en función del número de roundtrips. (b) Respuesta de fase del mismo. . . . . .

4.19. Característica temporal del coeficiente de reflexión $\Gamma_{a}(t)$. La reflectividad de las FBGs es del 75\%. (a) Módulo al cuadrado del coeficiente de reflexión del subsistema. (b) Respuesta de fase del mismo. . . . . . . . . . . . . . . . 
4.20. (a) y (c) Reflectividad de la cavidad auxiliar para $\left|\rho_{\text {mirror }}\right|^{2}=0,16$ y 0,6 , respectivamente. (b) y (d) Fase del coeficiente de reflexión de la cavidad auxiliar para $\left|\rho_{\text {mirror }}\right|^{2}=0,16$ y 0,60 , respectivamente. . . . . . . . . . 136

4.21. Modulación del coeficiente de reflexión definido para la cavidad auxiliar del sistema en función del tiempo de propagación para $\left|\rho_{\text {mirror }}\right|^{2}=0,75$. (a) Reflectividad. (b) Fase del coeficiente de reflexión de la cavidad auxiliar.

4.22. Inestabilidad producida por el aumento en la reflexión del semiespejo formado en el extremo de la SMF28 de la cavidad auxiliar. (a) Ancho temporal del pulso y potencia máxima del pulso en función del tiempo de propagación para $\left|\rho_{\text {mirror }}\right|^{2}=0,75$. (b) Evolución de la energía del pulso en función del tiempo de propagación, la cual permanece constante luego de un breve

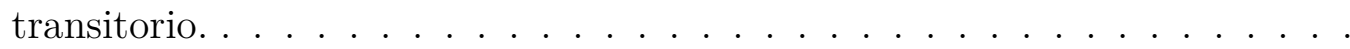

4.23. Evolución de la solución del sistema para $\left|\rho_{\text {mirror }}\right|^{2}=0,75$. La inestabilidad manifiesta en los cambios del ancho de pulso y la potencia de los mismos queda expresada en el plano potencia en función de la energía. (a) Evolución de la solución desde el inicio de la propagación. (b) Oscilación generada luego del transitorio inicial (aumento de la zona más densa de (a)) . . . . . . . . . 140

4.24. Frecuencia de la inestabilidad en función de la reflectividad del semiespejo. Para valores de $\left|\rho_{\text {mirror }}\right|^{2}$ levemente mayores al $60 \%$ las inestabilidades son oscilatorias, aunque generalmente se desarrollan de forma monótona, algunas hasta alcanzar otra condición estable. Para valores mayores, las inestabilidades de mantienen en el tiempo como se puede observar de la Fig. 4.23. Valores de $\left|\rho_{\text {mirror }}\right|^{2}>0,9$ complican el cálculo de la propagación mediante el SSM, debido al incremento del proceso no-lineal de la SPM. . . . . . . . 141

5.1. Caracterización espectral de las FBGs empleadas en la implementación del láser APM. La medida de la reflectividad de cada FBG se estima restando la potencia de la fuente al máximo valor de potencia reflejada. . . . . . . . 146 
5.2. Salida del láser APM en función de la potencia de bombeo: • es la componente CW observada a la salida del láser, $\boldsymbol{\Delta}$ está relacionado al modo de operación pulsado y $\square$ representa la potencia de salida del láser (CW + pulsado). La potencia del modo CW se obtiene calculando la diferencia entre el nivel de background y el nivel de fondo, obtenido cuando el fotodetector se encuentra apagado.

5.3. Emisión pulsada y estable del láser con potencia de salida uniforme, obtenida para diferentes valores de la longitud de la cavidad auxiliar $L_{A}$. (a) 1,976 m, (b) $0,988 \mathrm{~m}$, (c) $0,659 \mathrm{~m} \mathrm{y} \mathrm{(d)} 0,494 \mathrm{~m} \ldots \ldots \ldots \ldots$. . . . . . . . . 149

5.4. PSD para valores de $K$ enteros. Con "॰" se marcan las frecuencias múltiplos de la frecuencia de salida que deberían aparecer en cada caso bajo un régimen de operación APM (presenta los primeros 50 armónicos). (a) $K=1$, (b) $K=$ $2,(\mathrm{c}) K=3$, (d) $K=4$.

5.5. Emisión pulsada de salida para dos láseres APM con frecuencias de salida igual a $52 \mathrm{MHz}$. (a) $L_{M}=5,928 \mathrm{~m}, L_{A}=1,976 \mathrm{~m}(K=3)$. (b) $L_{M}=3,952$ $\mathrm{m}, L_{A}=1,976 \mathrm{~m}(K=2) \ldots \ldots \ldots \ldots \ldots$

5.6. (a) Medición del ancho de los pulsos de salida en función de la frecuencia de repetición del láser APM. (b) Comparación entre los ajustes realizados en [1] (con $\times$, empleando FBGs provistas por la Universidad de Valencia, España) y los evaluados en nuestro trabajo [2] (con o, utilizando FBGs comerciales marca O-ELAND). . . . . . . . . . . . . . . .

5.7. Salidas del láser para $1<K<2$ (relación de números coprimos). Sobre la izquierda se observan las mediciones temporales y del lado derecho la estimación de la PSD. (a) $K=4 / 3\left(f_{\text {laser }}=208 \mathrm{MHz}\right)$. (b) $K=7 / 4$

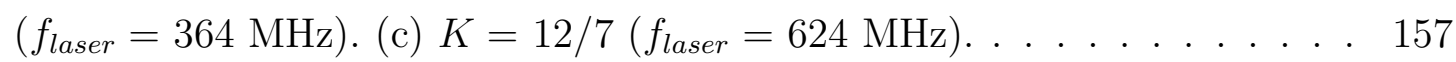


5.8. Potencias de salida del láser para $0<K<1$ (relación de números coprimos). Sobre la izquierda se observan las mediciones temporales. Del lado derecho, se muestra la PSD con las diversas componentes espectrales halladas en cada caso. (a) $K=11 / 13\left(\bar{T}_{\text {pulse }}=477 \mathrm{ps}\right)$. (b) $K=9 / 10\left(\bar{T}_{\text {pulse }}=588 \mathrm{ps}\right)$. (c) $K=21 / 22\left(\bar{T}_{\text {pulse }}=500 \mathrm{ps}\right) \ldots \ldots \ldots \ldots \ldots \ldots$. . . . . . . . . . . . . . . .

5.9. Frecuencias posibles y frecuencias medidas en función de $K$. (a) $L_{M}=1,976$ m ( $\square$ salidas estable, $*$ salidas estables observadas en [1], o salidas moduladas en potencia). (b) $2 L_{M}=3,952 \mathrm{~m}$. (c) $3 L_{M}=5,928 \mathrm{~m}$. En general, el número de frecuencias posibles de estos diagramas se incrementa con la longitud $L_{M}$. Para valores enteros pares de dicha longitud $\left(2 L_{M}, 4 L_{M}\right.$, etc. $)$ las frecuencias posibles para $2 L_{M}$ están incluidas en las frecuencias posibles halladas para $4 L_{M}, 6 L_{M}$, etc. Ídem para valores impares $\left(L_{M}, 3 L_{M}\right.$, etc. $) . \ldots . . . .160$

5.10. Simulación de la emisión pulsada del láser a una frecuencia igual a $52 \mathrm{MHz}$. (a) $2 L_{M}$ y $K=2$ (con ancho de pulso igual a $3,46 \mathrm{~ns}$ ); (b) $3 L_{M}$ y $K=3$ (con ancho de pulso igual a $3,48 \mathrm{~ns}$ ). . . . . . . . . . . . . . . . 162

5.11. Eficiencia de conversión del láser en función de la frecuencia de salida, para los casos numéricos y experimentales analizados. . . . . . . . . .

5.12. (a) Densidad espectral de potencia medida a la salida del láser APM. El ancho de banda de la emisión generada por el sistema láser implementado es FWHM $\approx 20$ pm. (b) Estimación de la PSD mediante el uso de las características de reflexión de las FBGs mostradas en la Fig. 5.1. Se consideran los mecanismos de pérdida y ganancia de una cavidad Fabry-Perot para un nivel de excitación pequeño (resultando en la curva de ASE del medio activo filtrado por las FBGs). . . . . . . . . . . . . . . . . . .

6.1. Esquema de calculo por SSM. La longitud de FO es dividida en un numero grande de segmentos de ancho $h$. Dentro de estos segmentos, el efecto de la no-linealidad es incluida en la mitad del plano, como se muestra con la linea de trazos. 


\section{Índice de tablas}

3.1. Diagrama de retardos especificado por las FBGs para un sistema OBF de 3 ángulos y un PAA formado por 4 antenas isotrópicas. La primera fila indica los 4 retardos $\left(t_{n}\right.$ con $n=1,2,3$ y 4$)$ debidos a las FBGs ubicadas en una posición de referencia $(\Delta z=0) \ldots \ldots \ldots \ldots$

3.2. Ubicación espacial de las FBGs para un sistema OBF de 3 ángulos y un PAA formado por 4 antenas isotrópicas. La primera columna, ángulo $\theta_{3}=0^{\circ}$, expresa el punto de referencia desde donde se miden las distintas longitudes de FO entre las FBGs del OBF. . . . . . . . . . . . . . . .

4.1. Parámetros principales del sistema láser de cavidades acopladas que opera en régimen APM. Entre estos se destacan, por su importancia, los parámetros físicos de las FBGs y la dispersión y no-linealidad de la FO dopada con $\mathrm{Er}^{3+}$. La determinación de dichos valores es importante para lograr una correcta representación de la salida del láser mediante los modelos numéricos implementados. . . . . . . . . . . . . . . . . . . . 115 


\section{Resumen}

En el presente trabajo de tesis doctoral se estudian y analizan métodos analíticos aplicados a la generación y el procesamiento de señales ópticas mediante distintos dispositivos y sistemas de fibra óptica. Este trabajo se puede dividir en dos partes. En la primera parte, se estudian diversos dispositivos fotónicos basados en fiber Bragg grating (FBG) para su aplicación en la generación de señales ópticas en forma de pulsos de luz coherente mediante un diseño adecuado de una cavidad Fabry-Perot, el procesamiento analógico mediante sistemas interferométricos, la generación de líneas de retardo y la caracterización de señales ópticas en intensidad y fase mediante la implementación en el dominio óptico de una de las propiedades de la función de distribución de Wigner. La segunda parte de esta tesis se concentra en el modelado y caracterización de un láser de fibra óptica formado por dos cavidades acopladas, el cual opera bajo el régimen additive-pulse mode locking (APM). Se propone un modelo analítico que resuelve la propagación de los campos eléctricos en ambas cavidades del láser. Este modelo, definido mediante un sistema de ecuaciones nolineales y acopladas, se resuelve a través un algoritmo que permite estimar la salida con un error pequeño. Mediante este nuevo algoritmo, basado en el split-step method (SSM) de paso variable, se realizan análisis diversos, tales como: estabilización del régimen pulsado y continuous wave $(\mathrm{CW})$ del láser por medio de un proceso de ruido gaussiano aditivo, acople de los campos eléctricos generados para distintas condiciones y un análisis de las posibles inestabilidades del sistema. Finalmente, se muestran mediciones experimentales y se realiza una comparación de los resultados obtenidos de forma teórica y experimental, encontrando una muy buena concordancia entre ellos. 


\section{Índice general}

$\begin{array}{ll}\text { Agradecimientos } & 5\end{array}$

Acrónimos

Glosario $\quad$ V

Índice de figuras

Índice de tablas $\quad$ XIX

Resumen

1. Introducción 1

2. Propagación de señales por una FO 13

2.1. Procesos lineales involucrados en la propagación . . . . . . . . . . . . . 17

2.1.1. Mecanismos de absorción . . . . . . . . . . . . . . . 17

2.1.2. Características de la dispersión . . . . . . . . . . . . 20

2.2. Introducción a los procesos no-lineales . . . . . . . . . . . . 25

2.2.1. Automodulación de la fase . . . . . . . . . . . . . . . . 25

2.2.2. Principios del régimen solitónico . . . . . . . . . . . . . 28

3. Modelado de filtros con FBGs y sus aplicaciones $\quad 31$

3.1. Modelado de FBGs . . . . . . . . . . . . . . . . . . . . . . . . . . . . 32 
3.1.1. FBGs de período uniforme . . . . . . . . . . . . 36

3.1.2. FBGs de período no-uniforme (LCFBG) $\ldots \ldots \ldots$

3.2. Aplicaciones . . . . . . . . . . . . . . . . . . . . 43

3.2.1. Cavidad Fabry-Perot de un láser que opera en régimen Q-switch . . 43

3.2.2. Sistemas de modulación de la intensidad mediante filtros interferométricos MI y MZI . . . . . . . . . . . . . . 56

3.2.3. Implementación de líneas de retardo ópticas para un sistema de OBF 74

3.2.4. Método de caracterización de pulsos basado en un dispositivo fotónico y dispersivo . . . . . . . . . . . . . . . . . . . . . 84

4. Modelado de un láser APM pasivo de cavidades acopladas 93

4.1. Introducción . . . . . . . . . . . . . . . . . . . . . . . . . . . . . 94

4.1.1. Diseño de las cavidades . . . . . . . . . . . . . . . . . . . 96

4.1.2. Propiedades espectroscópicas del $\mathrm{Er}^{3+} \ldots \ldots \ldots \ldots$. . . . 98

4.1.3. Régimen de operación mode locking . . . . . . . . . . . . . . . . . 102

4.2. Descripción del modelo teórico . . . . . . . . . . . . . . . . . . . . 105

4.2.1. Análisis de la ecuación del sistema . . . . . . . . . . . . . . 110

4.3. Modelo numérico implementado mediante SSM _ . . . . . . . . . . . . 117

4.3.1. Establecimiento del régimen pulsado y $\mathrm{CW}$ mediante un proceso de ruido aleatorio . . . . . . . . . . . . . . . . . . . . . . . . . . 122

4.3.2. Acoplamiento de los campos provenientes de ambas cavidades del láser APM . . . . . . . . . . . . . . . . . . . . . 126

4.3.3. Efecto de la dispersión en la cavidad activa . . . . . . . . . . . . 130

4.3.4. Coeficiente de reflexión no-lineal de la cavidad auxiliar . . . . . . . 133

4.3.5. Inestabilidades del sistema . . . . . . . . . . . . . . . . . . . 137

4.4. Discusión de los resultados numéricos . . . . . . . . . . . . . . . . . . . . . 141

5. Implementación del láser APM de cavidades acopladas 143

5.1. Descripción de la configuración experimental . . . . . . . . . . . . . . . . . 144 
5.2. Mediciones experimentales . . . . . . . . . . . . . . . . . 145

5.2.1. Relaciones enteras de longitud . . . . . . . . . . . . . . . . . . . 148

5.2.2. Generalización para distintas relaciones de longitud . . . . . . . . 156

5.3. Comparación teórico-experimental de los resultados obtenidos . . . . . . . 161

$\begin{array}{lr}\text { 6. Conclusiones } & 167\end{array}$

$\begin{array}{ll}\text { Apéndices } & 175\end{array}$

$\begin{array}{ll}\text { Referencias } & 196\end{array}$ 


\section{Capítulo 1}

\section{Introducción}

Luego de varios años de investigación en la búsqueda de nuevos medios físicos para los sistemas de comunicación, a mediados de la década del '70 comenzaron a consolidarse los sistemas de fibra óptica (FO) como medio de transmisión de señales de luz. La ventaja principal del empleo de la FO radica en su baja atenuación por kilómetro, usando una longitud de onda adecuada en las regiones espectrales del infrarroja cercana (NIR) y la infrarrojo de onda corta (SIR), lo que permite realizar enlaces largos sin necesidad de amplificación y/o regeneración de las señales ópticas transmitidas. Otras ventajas importantes respecto a otros medios físicos de transmisión, como por ejemplo el cable coaxial, son: ancho de banda muy grande (al menos 1000 veces superior), precio muy bajo considerando el ancho de banda a transportar, inmunidad frente a fuentes de interferencia electromagnética (EMI) externas, seguridad en el transporte de información, peso y dimensiones notablemente inferiores a las de un cable metálico y la posibilidad de reemplazar a cientos de estos con igual capacidad de transporte, entre otras. Las FOs son actualmente una alternativa concreta para suplir todas las deficiencias que presentan los medios físicos tradicionales [3]. En el campo de las comunicaciones, diversas líneas de investigación han sido dirigidas al desarrollo de dispositivos y componentes destinados a potenciar la capacidad de transmisión, creando sistemas transparentes donde la información es transmitida, compensada, e inclusive procesada por elementos totalmente ópticos $[4,5]$. La utilización de amplificadores 
ópticos y compensadores de dispersión desarrollados con tecnología de FOs ha permitido reducir las distorsiones introducidas por el canal y la bit error rate (BER) en sistemas de comunicación digitales de alta tasa de transmisión (mayores a 10 Gbps), ampliando la extensión de los enlaces y evitando los inconvenientes producidos por la conversiones optoeléctrica (OE) y electro-óptica (EO), empleadas para eliminar dichas distorsiones en el dominio eléctrico, mediante un proceso de regeneración de los pulsos. Las distorsiones principales que introduce un canal de FO son: la dispersión cromática (CD), la dispersión por modo de polarización (PMD) y las no-linealidades, como la automodulación de fase (SPM), el mezcla de cuatro ondas (FWM) y la modulación cruzada de la fase (XPM), entre otras. En general, los procesos de dispersión introducen una distorsión en el dominio del tiempo de los pulsos transmitidos por una FO, ensanchándo o comprimiéndo dichos pulsos segun el caso y, modificando la interferencia intersímbolo (ISI) medida en el receptor. El incremento en la potencia de las señales ópticas transmitidas se traduce en un aumento de las no-linealidades, las cuales introducen distorsiones en el dominio de la frecuencia, generando muchas veces productos de intermodulación y efectos de interferencia entre canales, lo que produce un aumento notable de la BER en el receptor. Otros factores que contribuyen al empobrecimiento de la transmisión son las fuentes de ruido aleatorio como el ruido producido por las fuentes láser en los transmisores, el ruido fotónico en las diversas fibras que componen un enlace, la emisión espontánea amplificada (ASE) generada en los amplificadores ópticos, el ruido de los detectores y de las diversas etapas de electrónica que conforman los receptores [6]. De esta forma, los distintos componentes intercalados en un enlace de FO (láseres, amplificadores y compensadores) en conjunción con las características del receptor (ancho de banda y figura de ruido, entre otros), definen las características de la señal recibida. A pesar de todos estos inconvenientes, existen diversas técnicas que se emplean con el fin de reducir los efectos del deterioro de las señales propagadas por una FO (predistorsión de las señales en el transmisor, ecualizaciones de la respuesta del canal y procesamiento en el dominio óptico). Una técnica utiliza un proceso de codificación de los pulsos ópticos transmitidos mediante un sistema de procesamiento digital (en el dominio eléctrico) para corregir errores, implementar ecualizadores y realizar predistorsiones, entre 
otras [7-9]. Otra se basa en el procesamiento de las señales mediante dispositivos ópticos, como los ecualizadores y sistemas compensadores de la dispersión, FOs con dispersión desplazada y con dispersión opuesta, filtros cuyas características ópticas son programables, etc. [10-13]. Cada una de estas técnicas presenta ventajas y desventajas que dependen de cada aplicación en particular, aunque la tendencia general es desarrollar sistemas completamente ópticos.

En el marco de los dispositivos fotónicos orientados al procesamiento de señales ópticas, una red de Bragg grabada en fibra (FBG) fotosensible fabricada mediante la incidencia de un diagrama de interferencia y empleando una fuente láser cuya longitud de onda se encuentra en la zona espectral del ultravioleta (UV) es un tópico de gran interés tecnológico. Estos son aplicados tanto en la industria para el sensado de variables del mundo físico (debido a su gran sensibilidad, flexibilidad de rango dinámico e inmunidad a fuentes de EMI externas), como en los sistemas de comunicación ópticos para filtrar, ecualizar y/o compensar la CD en canales de multiplexación por división de longitud de onda (WDM) [10]. La fabricación de las FBGs se basa en una modulación del índice de refracción en el núcleo de una FO de forma localizada (generalmente del orden del centímetro) por medio de un patrón de interferencia generado con luz UV, logrando estructuras de grabado de distinto tipo, lo cual permite obtener características y funcionalidades diferentes. En general, las FBGs son usadas como filtros ópticos, ecualizadores de canales de WDM, compensadores de CD y TOD, en sistemas add-drop para encaminar señales en enlaces WDM y en diversos sistemas fotónicos. Debido a su inherente inmunidad a la EMI, se aplican ampliamente en el desarrollo de sensores de distintas magnitudes físicas como: temperatura, presión, deformación y determinación de las propiedades ópticas de los materiales, entre otras. Muchos de estos sensores emplean las propiedades espectrales de reflexión y transmisión de las FBGs, ya que presentan muy bajas pérdidas de inserción y pueden trabajar con anchos de banda angostos (10 GHz o menores).

A partir del desarrollo de FOs dopadas con tierras raras en la década del '80, surgieron los amplificadores ópticos y de forma conjunta una amplia gama de fuentes de luz láser y emisores súperradiantes (fuentes de baja coherencia) que son empleados en los sistemas 
actuales. Los amplificadores de FOs dopadas han revolucionado el campo de las comunicaciones ópticas eliminando el uso de repetidores electrónicos en enlaces WDM de alta capacidad como: 10 Gbps, 40 Gbps y los más actuales de 100 Gbps. El desarrollo de nuevos medios ópticos amplificadores que utilizan distintos dopantes $\left(\mathrm{Er}^{3+}\right.$ e $\mathrm{Yb}^{3+}$ entre otros), aún en estudio, junto a técnicas de amplificación paramétrica ampliará el rango espectral de transmisión y la capacidad de los sistemas [14,15]. Estos esquemas de amplificación son desarrollados a partir de las propiedades no-lineales de las FOs, como los producidos por los procesos paramétricos y del esparcimiento Raman estimulado (SRS) [16]. El desarrollo de fuentes de luz coherente basadas en amplificadores ópticos y medios de realimentación, como espejos y filtros como las FBGs, hizo posible la aparición de los sistemas láser de FO y de onda continua (CW) como láseres con salidas pulsadas de diversas características (Q-switch y mode locking, entre otros). Estas fuentes de luz coherentes son de particular interés en el maquinado de materiales y el estudio de procesos lineales y no-lineales producidos durante la propagación de las señales por una FO. Los sistemas láser de FO y que funcionan bajo un régimen de suma de pulsos por enganche de modos (APM) ha sido un tópico de intensa investigación y desarrollo en los últimos años. La mayor parte de estos sistemas están basados en la idea de la generación de pulsos ultracortos y peines de frecuencia ópticos para diferentes aplicaciones relacionadas a las comunicaciones y en nuevas técnicas empleadas en metrología óptica [17-20]. Los láseres pasivos APM aparecieron primero en configuraciones de óptica tradicional, o bien del tipo bulk-optics, aunque presentaban muchos problemas de alineación. Luego fueron mejorados notoriamente al ser realizados con diferentes tipos de FOs dopadas y filtros, por ejemplo, empleando FO dopada con $\operatorname{Er}^{3+} \mathrm{y}$ FBG [21-23]. Estos sistemas permiten alcanzar potencias de pulso moderadas, del orden de las decenas o centenas de miliwatts, con anchos temporales que alcanzan los picosegundos, y más cortos aún empleando diversas técnicas de compresión [24-27]. Potencias mayores son obtenidas mediante el uso de diversos sistemas de modulación, los cuales modifican las pérdidas o la ganancia dentro o fuera de la cavidad activa de los láseres. En la actualidad, se investigan y desarrollan láseres APM híbridos (que emplean ambos principios) y que son implementados con FOs de $\mathrm{Er}^{3+}, \mathrm{Er}^{3+} / \mathrm{Yb}^{3+}, \mathrm{Yb}^{3+}$ y double-cladding, las cuales permiten 
alcanzar potencias altas (mayores a $10 \mathrm{~W}$ ) con anchos de pulso temporales del orden de los femtosegundos [28-30]. Los métodos analíticos empleados en el modelado de este tipo de láseres varían dependiendo del nivel de aproximación de los resultados. Generalmente, la envolvente del campo eléctrico es calculada mediante una ecuación maestra que depende de parámetros simples como términos que expresan la ganancia y/o pérdida, coeficientes de dispersión y no linealidad de las FOs empleadas, y coeficientes de acople relacionados a la reflectividad y/o transmitancia de algún semiespejo [31,32]. Sin embargo, particularmente en el modelado de láseres de pulsos ultracortos y/o en sistemas de generación de peines de frecuencia en el dominio óptico, estos modelos no pueden ser aplicados debido a que los parámetros como la dispersión y las distintas no-linealidades dependen de la longitud de onda de las diversas componentes espectrales de las señales propagadas [33-35]. En estos casos, es necesario una adecuada representación de los diferentes perfiles de la dispersión, de los cambios en la velocidad de grupo y de los términos no-lineales relacionados con la propagación en las FOs, resultando en modelos mucho más complejos. En general, los modelos numéricos empleados para calcular la propagación de los campos eléctricos en un sistema láser se concentran en resolver la ecuación no-lineal de Schrödinger (NLSE) bajo condiciones de contorno particulares [16], utilizando el método de Runge-Kutta para encontrar una solución numérica en un modelo simplificado con parámetros simples [36]. Sin embargo, cuando las condiciones de contorno y los procesos involucrados requieren la aplicación de un modelo más complejo (bajo las condiciones anteriormente mencionadas), la aplicación de dicho método incrementa notablemente el costo computacional. Como una solución a este problema se emplea el método de pasos divididos (SSM), donde la propagación de los campos electromagnéticos se calcula mediante tramos o secciones de FO de longitud más pequeña, y cuyo valor es variable y depende de una cota de error admitida para el cálculo de la fase no-lineal (relacionada a diversos procesos de la FO como SPM, self-steepening y FWM, entre otros). Este método funciona muy bien y es flexible frente a la introducción de diferentes procesos no-lineales que se producen en las FOs, alcanzando soluciones precisas en menor tiempo. Además, se puede configurar fácilmente para funcionar con otros métodos de cálculo, como el método de la matriz de transferencia (TMM). Las aplicaciones 
de los láseres APM en el área de las comunicaciones están destinadas a la generación de fuentes de luz con trenes de pulsos ultracortos empleadas para transmitir señales de altas tasas de bit (mayores a 40 Gbps) con muy bajos niveles de jitter [17,37,38]; en metrología óptica se aplican en la determinación de parámetros lineales (como la dispersión cromática y de alto orden) y de los diversos procesos no-lineales de las FOs.

El presente trabajo de tesis doctoral comienza en el Capítulo 2 con un estudio de los principios de la propagación de pulsos de luz en FOs, basado en el cálculo analítico de la NLSE. La misma es presentada por medio de sus términos generales, concentrándonos luego en los términos de mayor interés para la realización de este trabajo. Se analizan distintos procesos lineales de las FOs como la atenuación y la dispersión en el marco de la propagación de pulsos gaussianos. Mediante el método numérico SSM, desarrollado en el Apéndice, se estudia la relación entre la dispersión y uno de los procesos no-lineales producidos en la propagación de pulsos por una FO (SPM), como uno de los mecanismos fundamentales para la generación y/o propagación de solitones. Estas estructuras de pulsos permiten describir la salida de diversos sistemas láser que funcionan bajo el régimen de operación mode locking, como los que serán discutidos posteriormente en el Capítulo 4. Mediante la implementación de un algoritmo basado en el SSM, el cual puede ser configurado, dependiendo el caso, para calcular la propagación mediante el uso de pasos fijos y/o variables, se resuelve la NLSE de forma numérica para un error de fase pequeño. Esta herramienta permitió estimar la propagación de un campo eléctrico en diversos dispositivos y sistemas de FO desarrollados a lo largo de este trabajo de tesis.

En la primera sección del Capítulo 3 se estudia la propagación de los campos eléctricos en las FBGs, mediante las ecuaciones de modos acoplados. Se analizan en particular las FBGs de período uniforme y con una modulación del índice de refracción sinusoidal, encontrando una expresión general para el coeficiente de reflexión, el cual resulta en una función compleja que depende de los parámetros constructivos de la FBG, algunos tales como: la longitud física y la máxima amplitud de la modulación del índice de refracción en la zona donde se produce el grabado. También se extiende el análisis de la propagación de los campos eléctricos para FBGs no-uniformes, donde una variación del período de grabado de forma 
lineal (LCFBG) es expresada como una sucesión de tramos o secciones de FBGs uniformes y cuyas características son alteradas en función de la longitud de propagación. El análisis y diseño de estos dispositivos de FO se realiza mediante el empleo del TMM, el cual es introducido en el Apéndice, y fue implementado de forma numérica para ser utilizado en este trabajo de tesis. De esta manera, este algoritmo se empleó en la caracterización espectral de diversos dispositivos fotónicos descritos a lo largo de los Capítulos 3 y 4 . Además, fue empleado también en el Capítulo 4 para resolver la propagación de los campos eléctricos en las FBGs que componen el sistema láser de cavidades acopladas. La importancia del método TMM proviene de su gran flexibilidad frente a diversos tipos de estructuras fotónicas, tales como interferómetro Fabry-Perot (FPI), FBG de distinto tipo y/o filtros de DFB, los cuales presentan propiedades interesantes en el diseño de sistemas láser. En la segunda sección del Capítulo 3 se proponen cuatro aplicaciones que están relacionadas a la generación y procesamiento de señales ópticas coherentes. La primera se centra en el diseño y caracterización de una única cavidad láser, lo que hizo posible la presentación de algunos resultados analíticos mediante un trabajo de mi autoría [39], compuesta por una FO dopada con $\mathrm{Er}^{3+}$ y dos FBGs de período uniforme con longitudes de onda de Bragg de valores levemente diferentes. Una de estas FBGs está adherida a un actuador electromecánico tipo piezoeléctrico (PZT), el cual permite producir un estiramiento local de la misma e inducir un cambio de la longitud de onda de Bragg de este dispositivo con el fin de alterar las pérdidas del láser de forma controlada. La longitud de la cavidad es ajustada para que las frecuencias equiespaciadas generadas por la misma coincidan de alguna manera con la respuesta electromecánicas del PZT, con el fin de obtener una emisión pulsada eficiente bajo un régimen de operación $Q$-switch. De esta manera, se obtienen potencias pico del orden de los cientos de miliwatts y frecuencias de repetición del orden de los kilohertz o megahertz dependiendo de las características geometricas del actuador piezoeléctrico, el cual presenta una carga mecánica mínima (FBG adherida al PZT). Una segunda aplicación emplea FBGs de período uniforme y acopladores de FO para generar la interferencia de señales ópticas coherentes y de forma controlada mediante dos configuraciones típicas un interferómetro Michelson (MI) y otro denominado interferómetro Mach-Zehnder (MZI) [40]. Se diseñó e 
implementó un MI mediante un acoplador y dos FBGs idénticas para obtener una señal de interferencia mediante la reflexión de los campos que llegan a las FBGs se propagan hacia una de las ramas de entrada del acoplador, alcanzando un ajuste adecuado del filtro. El mismo permite modificar la intensidad reflejada resultante mediante el control de una diferencia de camino óptico, por ejemplo, mediante un modulador de fase o por medio de un cambio en las longitudes de los tramos de FO empleados (en un rango limitado por la coherencia espacial) para conectar las FBGs al acoplador. De una forma similar a la primera aplicación, éste mecanismo permite alterar las pérdidas de un sistema láser, aunque obteniendo mayores velocidades de respuesta. Se implementó un MZI como una extensión del MI, agregando una funcionalidad extra: las pérdidas pueden ser alteradas mediante la interferencia de dos señales que se transmiten a un segundo acoplador de FO. Esta característica es utilizada en el diseño de láseres que trabajan en régimen mode locking de pulsos cortos y ultracortos $[29,41,42]$. Además, se realiza un estudio de las características del filtro obteniendo una mejora notable en la respuesta de la fase (relacionada a la diferencia de camino óptico) y se muestran dos condiciones de desajuste del mismo, obtenidas de forma experimental. La tercera utiliza múltiples FBGs de período uniforme como elementos de reflexión y para generar líneas de retardo puras de FO, las cuales son utilizadas en un sistema de procesamiento analógico para la formación de haces electromagnéticos o beamforming mediante un conjunto de antenas de radiofrecuencia. El procesamiento óptico realizado mediante las FBGs presenta al menos un par de ventajas, comparando con las implementadas mediante electrónica de radiofrecuencias y/o el procesamiento digital, ya que provee una mayor inmunidad frente a la fuentes de EMI externas y permite manejar señales de gran ancho de banda, mejorando notablemente la sensibilidad de estos sistemas. Se estudió un esquema básico formado por FBGs de distintas longitudes de onda, circuladores de FO, una fuente de luz láser de longitud de onda variable (en función de las FBGs) y un modulador de intensidad tipo Mach-Zehnder de $\mathrm{LiNbO}_{3}$. Se estableció un esquema de retardos temporales y la aplicación de los mismos mediante tramos de FO estándar de longitudes bien definidas, el cual es implementado mediante FBGs de alta reflectividad. Además, se realizó un analisis de los parámetros principales de diseño del sistema, donde el 
más importante, relacionado al estrabismo o squint del haz de radiofrecuencias generado, es producido por la desviación de la longitud de onda de Bragg de las FBGs utilizadas, como se muestra en un trabajo de mi autoría [43]. En la última aplicación, se presenta el diseño novedoso de un sistema de caracterización de señales ópticas mediante el uso de una FBG no-uniforme que presenta una variación lineal de su período (LCFBG), y que modelada a través del TMM presenta un retardo de grupo aproximadamente lineal. La caracterización de pulsos de diverso tipo, en intensidad y fase por medio de este sistema está basado en una propiedad de la función de distribución de Wigner (representación tiempo-frecuencia) y una aproximación que permite estimar dichos parámetros con un error pequeño, como se muestra en un trabajo de mi autoría recientemente enviado [44]. Este aporte es relevante debido a la sencillez de su implementación real y es un complemento de otros sistema comerciales y actuales de caracterización de pulsos cortos y ultracortos.

En el Capítulo 4 se realiza una breve introducción de los principios empleados en la amplificación de la luz mediante un proceso de emisión estimulada de radiación (LASER), empleando un medio activo, un mecanismo de excitación de dicho medio y un sistema de realimentación. Se establecen las características generales de los medios de ganancia [14], presentando algunos resultados preliminares para FOs dopadas con $\mathrm{Yb}^{3+}$ en un trabajo de mi propia autoría [45]. Se muestran los procesos de emisión y absorción en FOs dopadas con $\mathrm{Er}^{3+}$ y se estiman los procesos vinculados a un esquema láser de FO de cavidades acopladas, el cual es desarrollado a lo largo de este capítulo. Además, se introduce el concepto de realimentación de la emisión estimulada mediante el modelo de una cavidad Fabry-Perot, de forma similar a los dispositivos estudiados en el Capítulo 3 y se analizan otros diseños que permitirían generar trenes de pulsos ultracortos mediante la extensión del ancho de banda del sistema de realimentación (por ejemplo, empleando una configuración de anillo). En general, se introducen los diversos regímenes de funcionamiento de los sistemas láser y en particular se establecen las condiciones de funcionamiento del régimen mode locking para un láser de cavidades acopladas. En la segunda sección, se introducen las principales características del modelo analítico propuesto, se estudian y modelan las propiedades de la ganancia del sistema definida por los tiempos de vida medios del $\mathrm{Er}^{3+}$ (ganancia saturada), 
se estudia la propagación de los campos eléctricos dentro del láser mediante la definición de un operador que resuelve la NLSE (se suponen las pérdidas y/o ganancia de las FOs, la dispersión y la no-linealidad determinada principalmente por la SPM) y se establecen las condiciones de ajuste de la fase lineal y no-lineal bajo el régimen additive-pulse mode locking (APM). De esta manera, se realiza un análisis del sistema de ecuaciones no-lineales acopladas mediante una herramienta de cálculo simbólico, la cual permite obtener una primera aproximación de la solución bajo condiciones de contorno simples. Estos resultados numéricos junto con algunos resultados experimentales permitieron realizar otra publicacion de mi propia autoría [46]. El empleo de un segundo algoritmo que resuelve de forma numérica dicho sistema de ecuaciones mediante el SSM y una matriz de scattering permite obtener un modelo numérico alternativo, mucho más flexible que el anterior y que admite la representación compleja de las FBGs, en módulo y fase. Mediante este nuevo modelo, que se ajusta muy bien a los resultados obtenidos, como veremos luego en el Capítulo 5, se realizan distintos análisis con el fin de determinar el peso de cada uno de los procesos lineales y no-lineales involucrados en la determinación de la salida pulsada del láser. En un primer análisis se determina la presencia de dos regímenes de funcionamiento mediante la introducción de un pulso inmerso en ruido, observando que el régimen pulsado presenta una mayor ganancia que el régimen. Luego, se verifica la característica del ancho temporal de los pulsos de salida en función de la frecuencia de repetición de los mismos, la cual es típica de los sistemas mode locking y en nuestro caso se obtiene modificando la longitud de la cavidad auxiliar del láser [1]. El peso de la dispersión en la cavidad principal compuesta por una FO dopada con $\mathrm{Er}^{3+}$ y un par de FBGs es también analizado encontrando que la posición espectral relativa entre las FBGs es de gran importancia en la determinación del ancho temporal de los pulsos de salida del láser. En el último análisis, se estudia el acoplamiento de los campos eléctricos provenientes de ambas cavidades (régimen APM), por medio de la caracterización de un coeficiente de reflexión definido por los elementos que componen la cavidad auxiliar. Los resultados obtenidos permiten estimar la influencia de la no-linealidad de las FOs sobre las condiciones de estabilidad del sistema láser propuesto. Finalmente, se realiza una discusión de los resultados numéricos obtenidos. 
En el Capítulo 5 se muestran los resultados experimentales obtenidos de la implementación del láser de cavidades acopladas que funciona bajo el régimen APM, analizado en el Capítulo 4. En la primera sección se describen los dispositivos y características de la configuración experimental del sistema láser APM implementado. En una segunda sección se realiza una caracterización espectral de las FBGs empleadas mediante un analizador del espectro óptico y se obtiene la característica de transferencia definida como la potencia de los pulsos de salida en función de la potencia de excitación inyectada al medio activo. Luego, en una sección siguiente, se obtienen diversas señales de salida del láser para distintas longitudes de cavidad auxiliar, manteniendo una longitud de cavidad activa constante (relaciones de longitudes expresadas como números enteros), verificando una característica relacionada al ancho temporal de los pulsos como una función de la frecuencia de repetición de los mismos. Se extiende este procedimiento para relaciones de longitudes expresadas como números racionales, encontrando salidas de potencia uniforme y no-uniforme dependiendo de la elección de dicho valor. En estos casos, se hace un procesamiento digital de las señales obtenidas mediante el uso del periodograma de Welch para determinar la composición espectral de las señales generadas, encontrando una muy buena correlación para las condiciones de ajuste establecidas en el Capítulo 4. Se presenta un diagrama de valores de frecuencia de repetición posibles en función de la relación entre las longitudes de ambas cavidades y se expresan los distintos casos medidos. En la última sección del capítulo se lleva a cabo una comparación teórico-experimental de los resultados obtenidos en el Capítulo 4 y 5. El compendio y comparación de los resultados numericos y experimentales obtenidos resultaron en una publicación de mi autoría [2]. Finalmente se muestra una medida de la densidad espectral de potencia de las señales de salida del láser y se hace una comparación con las características espectrales de reflexión de las FBGs, empleando una estimación de las pérdidas y la ganancia del medio activo.

En el Capitulo 6 se presentan las conclusiones generales de este trabajo de tesis realizado y sus implicancias en investigaciones futuras.

Finalmente, en el Apéndice se describen los métodos utilizados y de forma separada se presentan las Referencias. 


\section{Capítulo 2}

\section{Propagación de señales por una FO}

La propagación de una onda electromagnética que se transmite por una FO se resuelve mediante el cálculo de las ecuaciones de Maxwell para una guía cilíndrica, dieléctrica e isotrópica $[4,16]$. La relación funcional entre el vector de polarización $\mathbf{P}=\mathbf{P}(\mathbf{r}, t)$ y el vector campo eléctrico $\mathbf{E}=\mathbf{E}(\mathbf{r}, t)$, donde $\mathbf{r}$ es un vector transversal al eje de la $\mathrm{FO}$ que describe el radio, permite definir los procesos lineales y no-lineales generados en la interacción luzmateria cuando un haz de luz que se propaga por una FO. El factor de proporcionalidad entre ambos se puede representar por medio de la constante de permitividad del vacío $\epsilon_{0}$ y el tensor de orden $j$ de la susceptibilidad del material $\chi^{(j)}(j=1,2, \ldots)$, o sea

$$
\mathbf{P}=\mathbf{P}_{L}+\mathbf{P}_{N L}=\epsilon_{0}\left(\chi^{(1)} \cdot \mathbf{E}+\chi^{(2)}: \mathbf{E E}+\chi^{(3)}: \mathbf{E E E}+\ldots\right) .
$$

El primer orden de dicho tensor $\chi^{(1)}$ incluye el valor del índice de refracción $n$ y el coeficiente de atenuación $\alpha$ de la FO, correspondientes al término de polarización lineal $\mathbf{P}_{L}$, mientras que el segundo orden del tensor $\chi^{(2)}$ es el responsable de los efectos no-lineales relacionados a la generación del segundo armónico y la suma de frecuencias. Sin embargo, como se supone que la FO es un material centro simétrico, el aporte de dicho tensor puede ser despreciado frente a los otros términos de la Ec. 2.1. De esta manera, los procesos nolineales son atribuidos principalmente al término de tercer orden $\chi^{(3)}$, el cual es responsable de la generación del tercer armónico, el FWM y la refracción no-lineal (dependencia del 
índice de refracción con la frecuencia y con la intensidad de la envolvente de campo eléctrico, o bien $\left.\tilde{n}\left(\omega,|E(z, t)|^{2}\right)\right)$. La naturaleza tensorial de $\chi^{(3)}$ puede afectar las propiedades de polarización de la luz por medio de la birrefringencia no-lineal, la cual es producida por diferencias de velocidad de propagación entre los diversos ejes transversales a la FO (contenidos en el plano $x y$ ). En general, los procesos no-lineales pueden ser clasificados como procesos paramétricos donde no hay transferencia neta de energía entre el material y la luz propagada (como la SPM, la XPM y el FWM entre otros); y procesos en los cuales sucede lo contrario (SRS y SBS). Dentro de los procesos paramétricos, la SPM (junto a la CD) juega un rol importante en la formación y propagación de solitones, técnica empleada en enlaces de comunicaciones de larga distancia, entre otras aplicaciones [47-50].

Empleando las ecuaciones de Maxwell bajo las condiciones establecidas anteriormente y utilizando la Ec. 2.1 se obtiene la ecuación de ondas que define la propagación de un vector de campo eléctrico como

$$
\nabla^{2} \mathbf{E}-\frac{1}{c^{2}} \frac{\partial^{2} \mathbf{E}}{\partial t^{2}}=\mu_{0} \frac{\partial^{2} \mathbf{P}_{L}}{\partial t^{2}}+\mu_{0} \frac{\partial^{2} \mathbf{P}_{N L}}{\partial t^{2}}
$$

donde $\mu_{0}$ es la permeabilidad del vacío y $c$ es la velocidad de la luz en el vacío.

Las soluciones de la Ec. 2.2 están definidas por medio de los diferentes modos de propagación admitidos por una FO. Las soluciones de estos modos son expresadas por medio de funciones de Bessel de primera especie $J_{0}$ para los modos del núcleo y de segunda especie $K_{0}$ para los modos del recubrimiento [4]. En general, se supone que se propaga un único modo por el núcleo de una FO (modo híbrido $\mathrm{HE}_{11}$ ) y esto sucede cuando $J_{0}(V)=0$, donde $V=k_{0} a\left(n_{1}^{2}-n_{2}^{2}\right)^{1 / 2}<2,405$ es la frecuencia normalizada, $k_{0}=2 \pi / \lambda_{0}$ es el número de onda, $a$ el radio del núcleo, $n_{1}$ y $n_{2}$ los índices de refracción del núcleo y el revestimiento, respectivamente. De esta manera, el vector de campo eléctrico propagado por una FO de longitud $z=L$ puede ser expresado en el domino de Fourier como

$$
\tilde{\mathbf{E}}(\mathbf{r}, \omega)=\hat{\mathbf{x}} F(x, y) \tilde{E}(z=0, \omega) \exp (j \beta(\omega) z)
$$

donde $\hat{\mathbf{x}}$ es el vector de polarización unitario, $F(x, y)$ es la función de distribución de 
campo modal (de perfil aproximadamente gaussiano en el plano $x y$ si $2<V<2,405$ ) y $\beta(\omega)$ es la constante de propagación. De forma análoga, se puede expresar el vector de campo magnético $\tilde{\mathbf{H}}(\mathbf{r}, \omega)$ por medio del vector polarización $\hat{\mathbf{y}}$ (ortogonal al vector $\hat{\mathbf{x}}$ ). En general, $F(x, y)$ depende de la frecuencia angular $\omega$, aunque esta dependencia puede ser despreciada cuando el ancho de banda de la señal cumple que $\Delta \omega \ll \omega_{0}$. Realizando un desarrollo en serie de Taylor alrededor de la frecuencia de trabajo $\omega_{0}$ de la constante de propagación resulta

$$
\beta(\omega)=\tilde{n}(\omega) \frac{\omega}{c}=\beta_{0}+\beta_{1}\left(\omega-\omega_{0}\right)+\frac{\beta_{2}}{2}\left(\omega-\omega_{0}\right)^{2}+\frac{\beta_{3}}{6}\left(\omega-\omega_{0}\right)^{3}+\ldots
$$

donde $\beta_{0}$ es un término de fase constante, $\beta_{1}=1 / v_{g}$ es un retardo de fase constante y que depende de la velocidad de grupo $v_{g} ; \beta_{2}$ y $\beta_{3}$ son coeficientes de dispersión de segundo y tercer orden (GVD y dispersión de tercer orden (TOD), respectivamente). En general, el régimen de trabajo se llama anómalo cuando el parámetro de dispersión de segundo orden es negativo $\left(\beta_{2}<0\right)$. El valor de este parámetro es importante en la propagación de trenes de pulsos por una FO a los efectos de limitar la ISI en el receptor.

Empleando las ecuaciones anteriores es posible encontrar una expresión para la NLSE, la cual permite resolver la propagación de un campo eléctrico transmitido por una FO mediante el modelo de la envolvente compleja del campo eléctrico (con frecuencia de trabajo $\left.\omega_{0}\right)$ y suponiendo la aproximación de variación suave de la envolvente (SVEA) de dicho campo. En general, en la representación mediante el modelo de la envolvente compleja se desprecia el aporte del término de fase constante $\beta_{0}$ (modelo de señal pasabanda), mientras que los términos de orden superior de la Ec. 2.4 pueden ser también despreciados para FO estándar, debido a que producen una distorsión muy pequeña comparada con los efectos introducidos por el valor del retardo de fase, la GVD y TOD. Incorporando además los términos correspondientes a algunos de los procesos no-lineales, los cuales deben ser tenidos en cuenta en el cálculo de la propagación de pulsos intensos y de corta duración, es posible expresar la NLSE como 


$$
\frac{\partial E}{\partial z}+\frac{\alpha}{2} E+\beta_{1} \frac{\partial E}{\partial t}+\frac{i \beta_{2}}{2} \frac{\partial^{2} E}{\partial t^{2}}-\frac{\beta_{3}}{6} \frac{\partial^{3} E}{\partial t^{3}}=i \gamma|E|^{2} E-\frac{\gamma}{\omega_{0}} \frac{\partial\left(|E|^{2} E\right)}{\partial t}-i \gamma \tau_{R} \frac{\partial|E|^{2}}{\partial t} E
$$

donde $E=E(z, t)$ es la envolvente compleja de un campo eléctrico de frecuencia $\omega_{0}, \gamma$ es el parámetro no-lineal de SPM y $\tau_{R}$ es un parámetro relacionado al (SRS) [16]. De esta manera, los términos del lado izquierdo están relacionados a los procesos lineales (atenuación y dispersión) y los términos del lado derecho de la Ec. 2.5 representan alguno de los procesos no-lineales como el SPM, self-steepening y el SRS, respectivamente.

Los procesos que no aparecen representados en la NLSE (por ejemplo, las no-linealidades de alto orden) son estudiados de forma separada debido a su complejidad. En general, los procesos involucrados en la Ec. 2.5 son generalmente determinados por los rangos de intensidad y duración temporal del campo eléctrico. En general, para pequeñas longitudes de FO y potencias menores a $1 \mathrm{~mW}$ los efectos no-lineales pueden ser despreciados. Sin embargo, cuando la intensidad de las señales ópticas transmitidas son incrementadas, los efectos no-lineales debidos al SPM, las no-linealidades de alto orden y el SRS comienzan a ser notorios en la distorsión de las señales ópticas. Por otra parte, la duración temporal de las señales propagadas es importante para limitar los efectos de la distorsión por GVD (relacionada a $\beta_{2}$ ), TOD $\left(\beta_{3}\right)$, dispersión de alto orden y PMD (debido a la birrefringencia de la FO), dado que las señales ópticas propagadas en forma de trenes de pulsos por una FO se ven temporalmente ensanchadas, produciendo un incremento de la ISI. Las distintas componentes espectrales de dichos trenes de pulsos son relevantes en la generación coherente de nuevas componentes espectrales por FWM (cuando el peso de los procesos nolineales es preponderante) y en la distorsión entre dichas componentes por XPM (debidos a los cambios de la velocidad de grupo con la longitud de onda). Finalmente, los resultados obtenidos dependen directamente de la forma de las señales propagadas, por lo tanto, un estudio de la propagación empleando solitones difiere de los resultados hallados para pulsos gaussianos. 


\subsection{Procesos lineales involucrados en la propagación}

Los procesos lineales involucrados en el estudio de la propagación están relacionados a diversos procesos de atenuación y la dispersión de las señales propagadas por una FO. El estudio de la atenuación es útil en el diseño de enlaces de comunicaciones para estimar la ubicación espacial de los amplificadores ópticos y/o de los regeneradores de señal (basados en etapas de conversión OE y EO). Una atenuación baja, del orden de $0,2 \mathrm{~dB} / \mathrm{km}$ en la banda $\mathrm{C}$ del espectro óptico $\left(\lambda_{0}=1550 \mathrm{~nm}\right)$ permite extender los enlaces a algunos cientos de kilómetros con amplificación, aunque dicha extensión está limitada por el valor de la mínima relación señal ruido (SNR) en el receptor, la tasa de transmisión y el valor de la dispersión de segundo orden de la FO. Por ejemplo, para transmitir 1 Gbps en una FO estándar (SMF28), utilizando una fuente de luz coherente de $\lambda_{0}=1550 \mathrm{~nm}$ el enlace se puede extender hasta $30 \mathrm{~km}$ aproximadamente sin necesidad de compensación de la dispersión. Si se emplea una fuente de luz coherente con longitud de onda $\lambda_{\text {zero-dispersion }}=1310 \mathrm{~nm}$ donde la dispersión es nula, la distancia del enlace anterior puede ser incrementada hasta varios cientos de kilómetros, siempre y cuando se desprecien los efectos de la atenuación. En comunicaciones, el conocimiento de los efectos de la dispersión es importante para limitar la ISI y la BER medida en el receptor. Además, permite definir las características de un régimen solitónico, relacionado a la interacción de fase lineal y no-lineal. A continuación se realiza un análisis de los principales procesos lineales producidos en la propagación de pulsos por una FO estándar bajo la condición de propagación de un único modo.

\subsubsection{Mecanismos de absorción}

El efecto de la atenuación de las señales ópticas propagadas por una FO está relacionada a distintos procesos de absorción del material y sus efectos son notorios en distancias largas (desde algunas decenas de kilómetros). Los procesos de absorción en la FO, cuando la misma es excitada con un haz de luz aproximadamente monocromático de longitud de onda entre 800-1600 nm, se pueden separar en dos grandes grupos: primero, la absorción producida por el material intrínseco $\left(\mathrm{SiO}_{2}\right)$ y, por otra parte, la absorción que introducen la 
impurezas. Las pérdidas producidas de forma intrínseca están vinculadas a los procesos de absorción en la región espectral del UV $(\lambda<0,4 \mu \mathrm{m})$, y de forma conjunta con los procesos que ocurren en la región del infrarrojo $(\lambda>7 \mu \mathrm{m})$, lo que deriva en un comportamiento similar a una parábola en función de la longitud de onda. Por otra parte, las impurezas introducidas en el proceso de fabricación permiten generar las FOs de índice escalón (como la FO estándar), mediante $\mathrm{GeO}_{2}, \mathrm{P}_{2} \mathrm{O}_{5}$ y $\mathrm{B}_{2} \mathrm{O}_{3}$, y la de otros elementos metálicos que introducen transiciones en el espectro de absorción. En la Fig. 2.1 se puede observar la rama izquierda de una característica parabólica y un pico de absorción importante en la zona espectral de 1,4 $\mathrm{mm}$, mientras que la rama derecha de la parábola no es comunmente especificada debido a que en FOs presenta una atenuación mayor. La transición correspondiente a 1,4 $\mu \mathrm{m}$ es debida a la absorción del ion $\mathrm{OH}$ presente en el proceso de fabricación de la mayoría de las FOs. En los últimos 15 años se fabrican FOs donde la absorción en dicha ventana espectral ha sido prácticamente eliminada mediante un control adecuado que permite limitar la presencia de agua en el proceso de fabricación. De esta manera, se amplia significativamente el rango espectral de transmisión de los nuevos sistemas de comunicación por FO, incrementando la capacidad de los mismos. Otro mecanismo de atenuación denominado esparcimiento Rayleigh es originado por las fluctuaciones microscópicas locales en la densidad del material, las cuales producen cambios aleatorios en el índice de refracción en una pequeña escala. Las imperfecciones en la guía de ondas (relacionadas a la interfaz núcleo-revestimiento) pueden ocasionar atenuaciones por esparcimiento Mie, debidas a las inhomogeneidades del índice de refracción sobre una escala mayor a las longitudes de onda (del orden del 1\%).

Bajo estas condiciones generales, la intensidad de un campo eléctrico $E(z, t)$ propagado en una FO puede escribirse como una relación entre las potencias (intensidad por unidad de área) de entrada y salida como

$$
P(z, t)=P_{0}(z=0, t) e^{-\alpha(\lambda) z}
$$

donde $z$ como ya hemos mencionado es la longitud de propagación, $\alpha(\lambda)$ es el coeficiente 


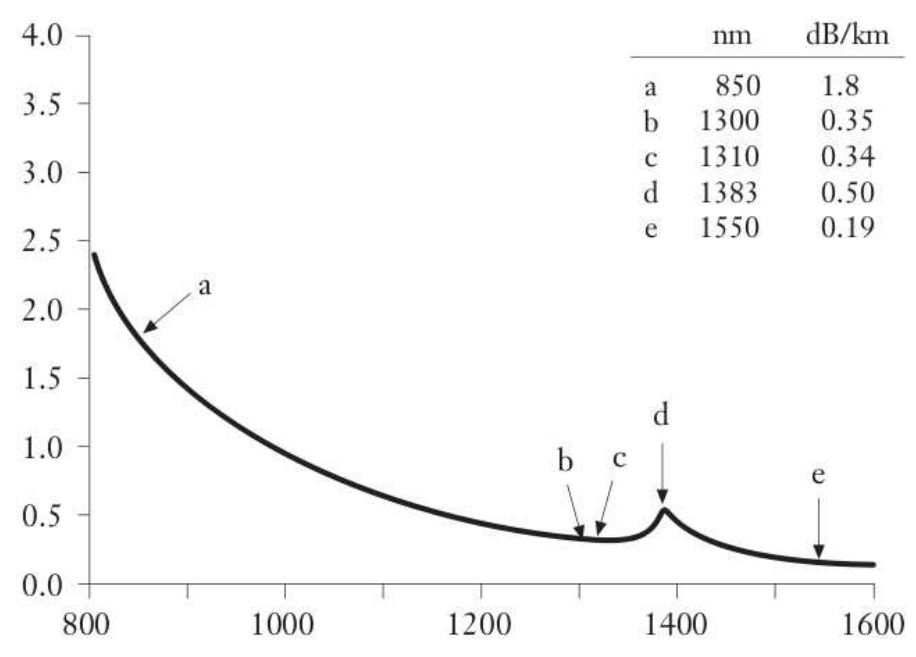

Figura 2.1: Atenuación de una FO estándar (SMF28 Corning) en función de la longitud de onda.

de atenuación y $P_{0}(z=0, t)=|E(0, t)|^{2} A_{\text {eff }}$ es la potencia a la entrada de la FO. Para FO estándar, un valor típico de atenuación es igual a $\alpha\left(\lambda_{0}\right)=0,19 \mathrm{~dB} / \mathrm{km}$ para una longitud de onda de trabajo $\lambda_{0}=1550 \mathrm{~nm}$, como se puede observar de la Fig. 2.1. Por ejemplo, un pulso de potencia unitaria que se propaga por una FO, la longitud que produce una atenuación de $3 \mathrm{~dB}$ (correspondiente a la mitad de la potencia a la entrada de la FO, o sea $P_{0} / 2=0,5$ ) es aproximadamente igual a $16 \mathrm{~km}$. De forma análoga para un tramo de FO de longitud idéntica, si se emplea una fuente de luz con una longitud de onda $\lambda_{0}=\lambda_{\text {zero-dispersion }}=$ $1310 \mathrm{~nm}$ donde $D=0$, el coeficiente de atenuación es igual a $0,34 \mathrm{~dB} / \mathrm{km}$ y el pulso de potencia unitaria sufriría una atenuación aproximada de 5,4 dB.

La importancia de la atenuación en sistemas láser que emplean FOs dopadas con $\mathrm{Er}^{3+}$,

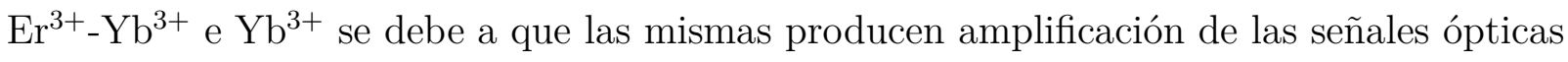
en dos bandas espectrales alrededor de 1,53 $\mu \mathrm{m}$ y $1 \mu \mathrm{m}$, respectivamente. Sin embargo, si estas FOs no son adecuadamente excitadas mediante un láser de semiconductor de intensidad mayor a algunas decenas de miliwatts, se produce una gran atenuación del orden de $30 \mathrm{~dB} / \mathrm{m}$ en $1530 \mathrm{~nm}$ o más (LIEKKI Er30-4, Er80-4, etc.). Aún cuando estas FOs son adecuadamente bombeadas, se pueden producir pérdidas adicionales si su longitud se incrementa más allá de los limites establecidos por la intensidad máxima de excitación [14]. 
Para la mayoría de los sistemas analizados en este trabajo, donde las señales son limitadas en banda (señales pasabanda), supondremos que no se produce distorsión lineal de amplitud debida a los cambios del coeficiente de atenuación de la FO con la longitud de onda, o sea, que todas las componentes espectrales sufren la misma atenuación. Los efectos de la atenuación en FO también son importantes en la determinación de los efectos no-lineales, los cuales dependen de la intensidad de las señales propagadas.

\subsubsection{Características de la dispersión}

El origen de la CD producida en una FO radica en el proceso de interacción de una onda electromagnética con los electrones de valencia del $\mathrm{SiO}_{2}$. Esta interacción luz-materia genera una dependencia del índice de refracción con la frecuencia $\tilde{n}(\omega)$, produciendo que las distintas componentes espectrales de un pulso de luz inyectado en una FO se propaguen a velocidades diferentes $v(\omega)=c / \tilde{n}(\omega)$, lo que origina una distorsión del mismo. Por lo tanto, si los efectos no-lineales no son importantes (si la potencia del pulso $P_{0}$ es pequeña), bajo un régimen de dispersión anómalo produce un ensanchamiento temporal de un tren de pulsos que se propaga por la FO (suponiendo que la fase inicial es nula). Despreciando los términos de la constante de propagación $\beta_{0}$ y $\beta_{1}$ y los términos de orden superior de la Ec. 2.4, la constante de fase depende únicamente del coeficiente de dispersión de segundo orden o $\beta_{2}$, el cual está relacionado con el parámetro de dispersión $D$ a través de

$$
D=\frac{\partial v_{g}^{-1}}{\partial \lambda}=-\frac{2 \pi c}{\lambda^{2}} \beta_{2}
$$

En general, el régimen de trabajo denominado anómalo $\left(\beta_{2}<0\right)$ se establece para la mayoría de las comunicaciones en las bandas C y L del espectro óptico, donde el parámetro de dispersión es positivo para una single-mode optical fiber (SMF), o sea, $D \approx 16$ ps/(km-nm) para $\lambda_{0}=1550 \mathrm{~nm}$. Sin embargo, otros sistemas funcionan en régimen de dispersión nula $D=0\left(\lambda_{\text {zero-dispersion }} \approx 1310 \mathrm{~nm}\right)$ donde los efectos de la TOD se vuelven mas importantes, o bien operan en régimen normal $\left(\beta_{2}>0\right)$. La Fig. 2.2 muestra la característica de la dispersión para una FO estándar SMF28 en función de la longitud de onda. Esta 


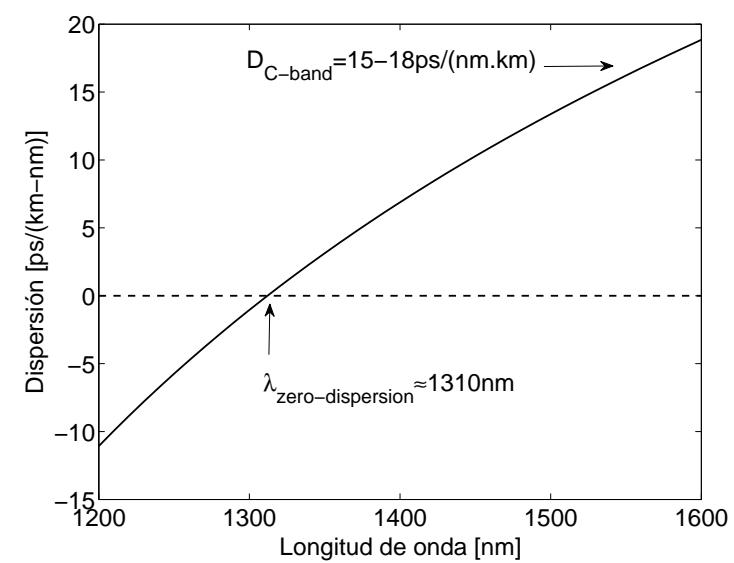

Figura 2.2: Dispersión de una FO estándar (SMF28 Corning) en función de la longitud de onda, expresada en nanometros.

es calculada mediante una ecuación $D(\lambda)=S_{0}\left(\lambda-\lambda_{0}^{4} / \lambda^{3}\right) / 4$ que brinda el fabricante de la FO, donde el valor de la pendiente de la dispersión en $1310 \mathrm{~nm}$ es igual a $S_{0}=0,086$ $\mathrm{ps} /\left(\mathrm{nm}^{2} \cdot \mathrm{km}\right)$.

El análisis de la dispersión de un pulso normalizado a la entrada $U(z, \tau)$ de una $\mathrm{FO}$ se puede realizar mediante la Ec. 2.5, donde dicho pulso se puede definir como

$$
E(z, \tau)=\sqrt{I_{0}} e^{-\alpha z / 2} U(z, \tau)
$$

donde $\tau=\left(t-z v_{g}^{-1}\right) / T_{0}$ es la variable tiempo normalizada, $I_{0}=\max (I(0, t))$ es la intensidad máxima de entrada y $T_{0}$ es el ancho temporal medido a $1 /$ e del máximo de intensidad. Despreciando el aporte de los términos debidos a la atenuación, la TOD y los términos no-lineales de orden superior de la NLSE, la misma se puede reescribir como

$$
i \frac{\partial U}{\partial z}=\frac{\operatorname{sgn}\left(\beta_{2}\right)}{2 L_{D}} \frac{\partial^{2} U}{\partial \tau^{2}}-\frac{e^{-\alpha z}}{L_{N L}}|U|^{2} U
$$

donde $U=U(z, \tau)$ es el campo normalizado, $L_{D}=T_{0}^{2} /\left|\beta_{2}\right|$ es la longitud efectiva de dispersión y $L_{N L}=\left(\gamma I_{0}\right)^{-1}$ es la longitud efectiva no-lineal (donde $\gamma=2 \pi n_{2} /\left(A_{e f f} \lambda\right)$ es el parámetro no-lineal, $n_{2}$ es el coeficiente Kerr y $A_{\text {eff }}$ es el área efectiva de la FO la cual queda definida por el diámetro de campo modal para una condición de propagación de un 
único modo, como mencionamos anteriormente). Ambas longitudes efectivas permiten estimar los límites donde los efectos de la dispersión y de las no-linealidades son importantes. Mientras que la longitud de propagación $L$ sea mucho menor a las longitudes efectivas mencionadas, ambos efectos pueden ser despreciados. Suponiendo que los efectos no-lineales son pequeños, el segundo término de la Ec. 2.9 es despreciable y no se tiene en cuenta para el cálculo de la propagación. En ese caso, se encuentra una solución de la Ec. 2.9 de una forma sencilla aplicando la transformada de Fourier a un pulso de entrada, multiplicando por la exponencial compleja $\exp \left(i \beta_{2} L \omega^{2} / 2\right)$ y antitransformando. De esta manera, se puede encontrar una solución $U(z=L, \tau)$. El factor de fase mencionado induce una variación lineal o chirp en la frecuencia de trabajo del pulso transmitido, el cual puede ser visto en el dominio del tiempo si se expresa el resultado de forma analítica [4] (no se observa directamente mediante la representación de la envolvente compleja del campo eléctrico). En este caso, como la exponencial compleja sólo afecta a la fase del pulso transformado, la intensidad del mismo permanece inalterada para cualquier valor de $z$. En general, se puede analizar la propagación de un pulso gaussiano normalizado $U(z=0, \tau)=\exp \left(-\left(\tau / T_{0}\right)^{2 m} / 2\right)$ con fase inicial nula (donde $m$ es un número entero que determina el orden de la gaussiana) y ancho $T_{0}=1$ ns. En la Fig. 2.3(a) y (b) se muestra la evolución temporal y espectral del pulso gaussiano $(m=1)$ mencionado, a medida que se propaga por una FO de longitud igual a $2 L_{D} \approx 40 \mathrm{~km}$, donde $\delta f=f-f_{0}$ y $f_{0}$ es la frecuencia óptica de trabajo. El cambio de la intensidad y el ancho temporal de dicho pulso en función de la longitud de propagación se muestra en la Fig. 2.4. Se puede observar que los efectos de la dispersión son importantes para longitudes de FO grandes (mayores a $L_{D}$ ). Sin embargo, para cualquier valor de la longitud de propagación que cumpla con $L<<L_{D}$ se puede garantizar que el efecto de la dispersión es despreciable. En general, como la Ec. 2.9 no tiene solución analítica cerrada se calcula mediante métodos numéricos (SSM y diferencias finitas) [16].

Los procesos de la dispersión de alto orden, en general son importantes en sistemas de transmisión de WDM que poseen distintas etapas de compensación de la dispersión de segundo orden. En particular, si la longitud de onda de trabajo es próxima a $\lambda_{\text {zero-dispersion, la }}$ TOD juega un papel importante. Una manera de evaluar el peso relativo entre la dispersión 


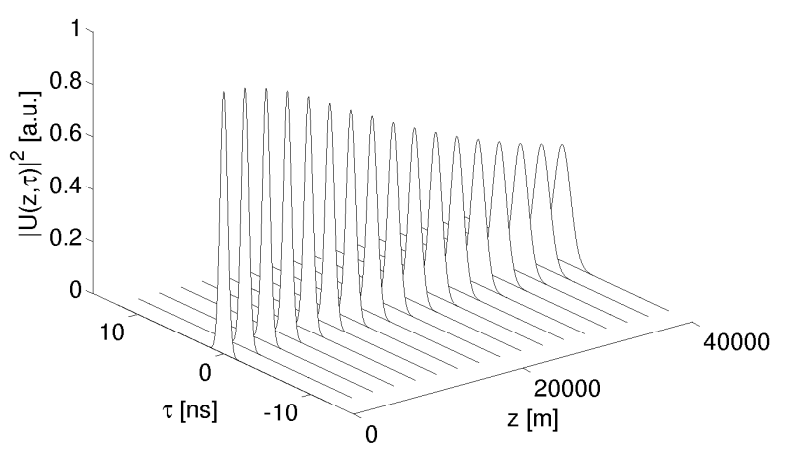

(a)

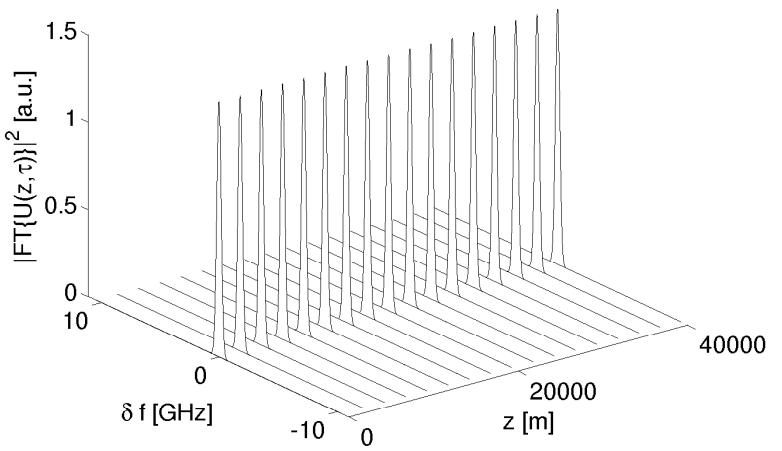

(b)

Figura 2.3: Propagación de un pulso gaussiano real de ancho $T_{0}$ sobre una FO de longitud $2 L_{D}$. El efecto de la dispersión bajo el régimen anómalo produce un ensanchamiento temporal del pulso a medida que se propaga por la FO, mientras que el espectro de dicho pulso resulta inalterado. (a) Evolución temporal en función de la longitud de propagación. (b) Densidad espectral de energía del pulso propagado.

de segundo y tercer orden es definiendo una longitud efectiva de dispersión de alto orden $L_{D}^{\prime}=T_{0}^{3} /\left|\beta_{3}\right|$. La TOD es importante frente a la GVD cuando $L_{D}^{\prime} \leq L_{D}$, mientras que el signo de $\beta_{3}$ determina la ubicación temporal donde se encuentra la distorsión, respecto del valor de intensidad máxima.

Es importante mencionar que la GVD y la dispersión de alto orden en FOs dopadas con tierras raras pueden ser significativas en el cálculo de la propagación para valores de longitudes de onda donde se establece el proceso de emisión espontánea amplificada (ASE). En general, ésta es generada mediante un proceso de excitación o bombeo de dichas FOs mediante el uso de un láser de semiconductor y presenta un máximo para una longitud de onda cercana a los 1,53 um (FO dopada con $\mathrm{Er}^{3+}$ ), lo cual permite amplificar señales ópticas dentro de un rango dinámico de longitudes de onda del orden de las decenas de nanometro. En esta zona del espectro óptico, el parámetro de la dispersión se expresa como 


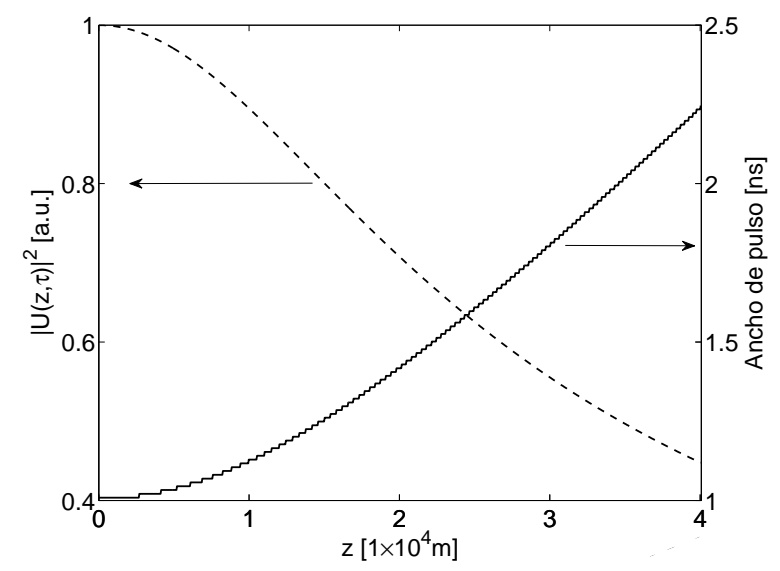

Figura 2.4: Evolución de la intensidad y el ancho temporal del pulso gaussiano, para una longitud de propagación $z=2 L_{D}$. La intensidad es representada en línea de trazos.

$$
D=D_{H}+D_{R}
$$

donde $D_{H}$ es el parámetro de dispersión de una FO estándar y $D_{R}$ está relacionado a la introducción de la tierra rara como material dopante. Este parámetro es muy importante en FOs dopadas con $\mathrm{Er}^{3+}$, ya que su valor cambia en módulo (hasta varios órdenes de magnitud) y signo, como una función de la longitud de onda [14]. Esta característica se hace más notoria en FOs altamente dopadas las cuales son empleadas en sistemas láser de alta potencia.

En transmisiones de pulsos de altas frecuencias de repetición, mayores al gigahertz, los efectos de la dispersión por modo de polarización sobre las señales propagadas se ven incrementados. La PMD se origina por un cambio de la velocidad de propagación de los ejes transversales a la FO (plano $x y$ ), debidos principalmente a la birrefringencia inducida en el proceso de frabricación de las FOs. El análisis de la PMD para estos casos es complejo debido a la característica aleatoria de la misma. De todas maneras, en la mayoría de los sistemas analizados en este trabajo de tesis nos limitaremos sólo al estudio de la GVD y su interacción con otros procesos como la SPM. 


\subsection{Introducción a los procesos no-lineales}

Los procesos no-lineales que están involucrados en la propagación de señales por una FO son originados principalmente por la presencia del tensor de tercer orden $\chi^{(3)}$ de la susceptibilidad del material, como ya hemos mencionado anteriormente. La mayoría de los procesos no-lineales, tales como el SPM, el self-steepening y las no-linealidades de alto orden, son introducidos en la NLSE por la dependencia que presenta el índice de refracción con la intensidad de las señales propagadas. En general, para pulsos con anchos temporales menores que 1 ps la dispersión de alto orden es comparable con la GVD y el proceso de self-steepening debe ser analizado con la SPM de forma conjunta. El proceso de la SPM relacionado al efecto Kerr es importante en el análisis de señales propagadas en FO dopadass con tierras raras, en enlaces de FO de larga distancia para comunicaciones (amplificadores de FO) y en diversos dispositivos fotónicos empleados en el diseño de láseres de FO. Las no-linealidades de alto orden (self-steepening y SRS entre otras) junto a la SPM producen distorsión de las señales de pulsos cortos y ultracortos, haciendo más complejo su estudio. En general, el análisis de otros procesos no-lineales como la XPM y el FWM se realiza de forma separada debido a que se expresan por medio de un sistema de ecuaciones acopladas, para las cuales se introduce la depedencia de diversos parámetros con la longitud de onda. Estos son de importancia en la descripción de las señales generadas mediante sistemas láser de FO que operan en régimen mode locking, los cuales presentan frecuencias discretas que verifican una condición de ajuste de la fase lineal y no-lineal [16]. A continuación se realiza un análisis de los efectos de la SPM en la propagación de un pulso gaussiano y se estudia la interrelación con la dispersión introduciendo los conceptos de la formación de solitones en FO, los cuales son analizados en el Capítulo 4.

\subsubsection{Automodulación de la fase}

La automodulación de fase (SPM) es un proceso no-lineal que depende de la intensidad de las señales propagadas. Suponiendo que los efectos de la dispersión y las no-linealidades de alto orden pueden ser despreciadas, o sea $L_{D} \gg L_{N L}$, la Ec. 2.9 puede ser simplificada de 


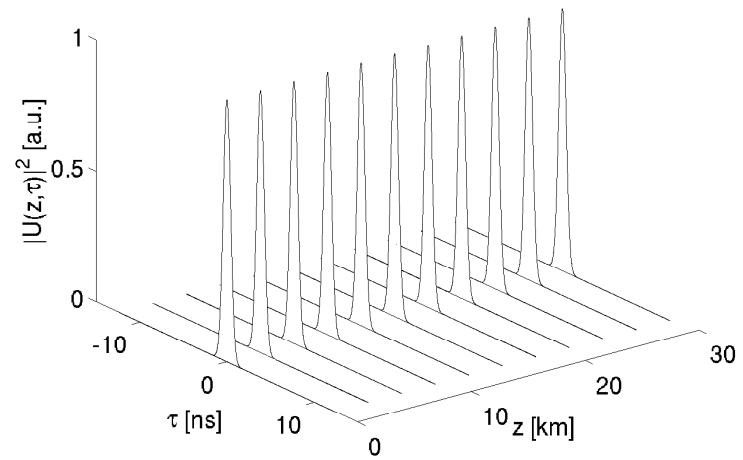

(a)

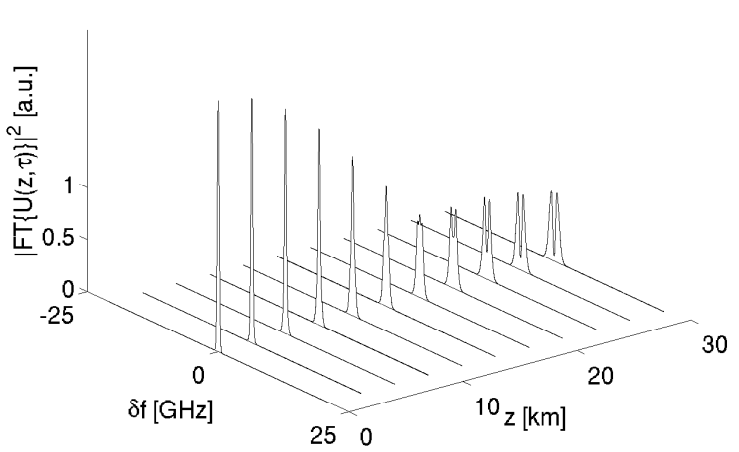

(b)

Figura 2.5: (a) Evolución del pulso propagado con SPM en el dominio temporal. (b) Densidad espectral de energía del pulso propagado.

la siguiente manera

$$
\frac{\partial U}{\partial z}=\frac{i e^{-\alpha z}}{L_{N L}}|U|^{2} U
$$

para una longitud de análisis $z=L>L_{N L}$ de FO, cuya longitud efectiva depende del parámetro no-lineal $\gamma \approx 1-5 \mathrm{~W}^{-1} \mathrm{~km}^{-1}$ para una SMF (dependiendo del valor del área efectiva $\left.A_{\text {eff }}\right)$. Se debe notar que si dicha área disminuye, el parámetro no-lineal se incrementa (FOs dopadas) fortaleciendo la distorsión de las señales por SPM en longitudes de FO menores. Suponiendo que la intensidad del pulso normalizado $|U(z, \tau)|^{2}$ no cambia con $z$, lo que implica guardar ciertos cuidados dependiendo del caso en análisis (por ejemplo, en FOs dopadas), la solución de esta ecuación diferencial queda definida por

$$
U(z, \tau)=U(z=0, \tau) \exp \left(i \phi_{N L}(z, \tau)\right),
$$

donde $\phi_{N L}(z, \tau)$ es la fase no-lineal, la cual puede ser expresada como

$$
\phi_{N L}(z, \tau)=|U(0, \tau)|^{2} \frac{\left(1-e^{-\alpha z}\right)}{\alpha L_{N L}},
$$




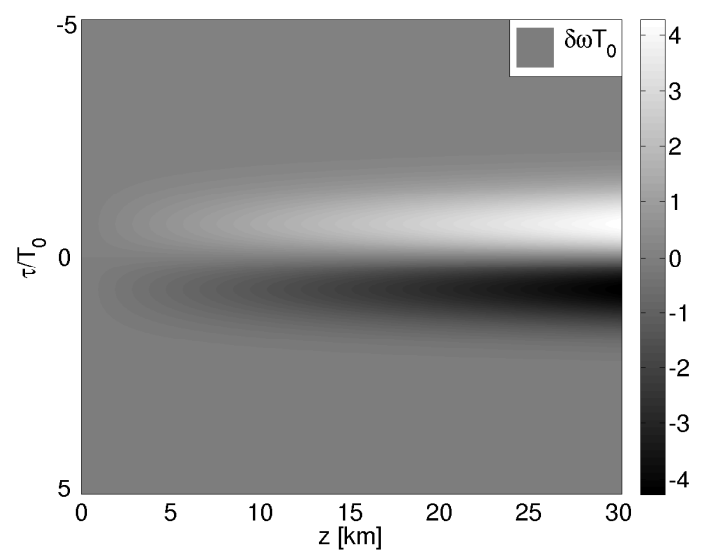

Figura 2.6: Representación del chirp inducido en el espectro $\left(\delta \omega T_{0}\right)$ por medio del proceso de la SPM. El eje de ordenadas describe la ubicación temporal de la distorsión en función de la longitud de propagación.

adquiriendo la forma del pulso analizado por medio de su intensidad y alcanzando un máximo para $\tau=0$. Un pulso propagado por una FO que presenta distorsión por la SPM no sufre ensanchamiento temporal debido a que $\phi_{N L}$ sólo induce un chirp de la fase en el dominio de la frecuencia de la señal. Suponiendo que se desprecia el efecto de la atenuación, entonces $\phi_{N L}(z, \tau)=|U(0, \tau)|^{2} z / L_{N L}$. De esta forma, en la Fig. 2.5(a) y (b) se muestra la propagación de un pulso en los dominios tiempo y frecuencia, observando un cambio en la intensidad y ancho de banda del mismo en el dominio transformado a medida que se propaga por una FO de longitud $z=5 L_{N L}$ con parámetro no-lineal $\gamma=2 \mathrm{~W}^{-1} \mathrm{~km}^{-1}$. El pulso de entrada a la FO empleado en el cálculo de la propagación es real y gaussiano, con ancho temporal $T_{0}=1 \mathrm{~ns}$ y tiene una potencia máxima igual a $P_{0}=100 \mathrm{~mW}$. En la Fig. 2.6 se muestra la magnitud del chirp inducido en la frecuencia $\delta \omega(z, \tau)=-\partial \phi_{N L} / \partial \tau$ en escala de grises, para cada valor de longitud evaluado. De estos resultados numéricos se puede observar que para valores de $\phi_{N L} \approx 1$ el efecto no-lineal produce un ensanchamiento espectral, mientras que para valores de $\phi_{N L} \ll 1$ (o bien para longitudes $z<5 \mathrm{~km}$ ) prácticamente no se produce distorsión por SPM. 


\subsubsection{Principios del régimen solitónico}

En general, en la propagación de pulsos por una FO los procesos lineales y no-lineales actúan de forma conjunta $[16,51]$. La dispersión de segundo orden junto a la no-linealidad introducida por la SPM es un ejemplo concreto. En este caso, un análisis de los efectos individuales no es del todo adecuado debido a que no se cumple el principio de superposición. Recordando la Ec. 2.9 y las definiciones de las longitudes efectivas de la dispersión no-lineal, se puede definir un nuevo parámetro $N=\left(L_{D} / L_{N L}\right)^{1 / 2}$ denominado orden solitónico. Este nuevo parámetro permite estimar el peso de los procesos lineal y no-lineal en la propagación de pulsos de luz por una FO. Cuando $N \ll 1$ el proceso dominante es la dispersión y en caso contrario se debe a la no-linealidad. En el límite, cuando $N=T_{0}^{2} \gamma I_{0} /\left|\beta_{2}\right|=1$ las longitudes efectivas de dispersión y no-lineal son iguales y ambos procesos resultan en la formación de un solitón. En estos casos, la expresión matemática que resulta de esta condición es del tipo secante hiperbólica [16].

El cálculo de la Ec. 2.9 no tiene una solución analítica, por lo cual se resuelve mediante métodos numéricos, como el SSM [16,52], descrito en el Apéndice. En las Figs. 2.7 (a) y (b) se muestra la propagación de un pulso gaussiano real en el dominio del tiempo y la frecuencia, respectivamente. La potencia del pulso de entrada fue igual a $P_{0}=100 \mathrm{~mW}$ y el ancho en el dominio del tiempo fue $T_{0}=10 \mathrm{ps}$. El coeficiente de dispersión de segundo orden y el parámetro no-lineal corresponden a una FO tipo SMF28 que opera en régimen anómalo $\left(\lambda_{0}=1550 \mathrm{~nm}\right)$. El ancho del pulso de entrada se eligió para conseguir un orden solitónico próximo a la unidad, o sea $N \approx 1$. En las Figs. 2.7 (c) y (d) se observa la variación de la intensidad y el ancho de los pulsos en el dominio del tiempo y la frecuencia, respectivamente. Se puede observar que en la propagación del pulso gaussiano sobre una FO de longitud igual a $L_{D} \approx L_{N L} \approx 6 \mathrm{~km}$ se produce una pequeña distorsión del mismo en ambos dominios, temporal y espectral (suponiendo un caso ideal sin atenuación).

Suponiendo que el pulso de entrada es real y presenta un perfil tipo secante hiperbólica, típico de un régimen solitónico (solución de la Ec. 2.9), se puede realizar el mismo análisis. En las Figs. 2.8 (a) y (b) se muestra la propagación de dicho pulso, cuyos parámetros (po- 


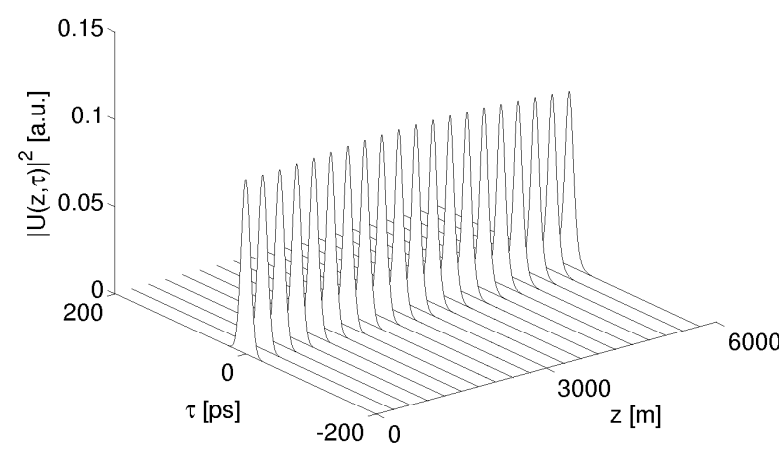

(a)

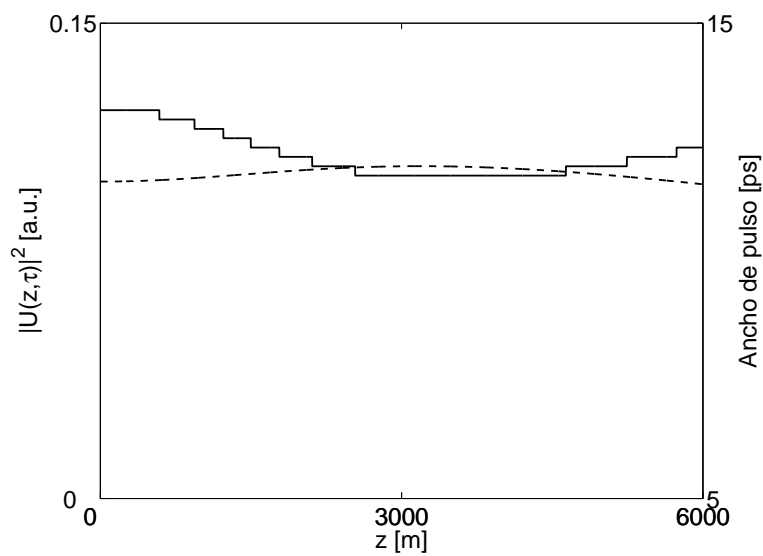

(c)

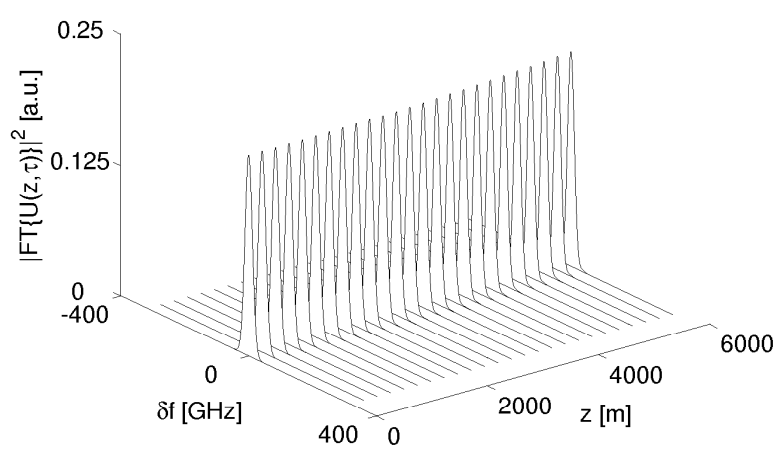

(b)

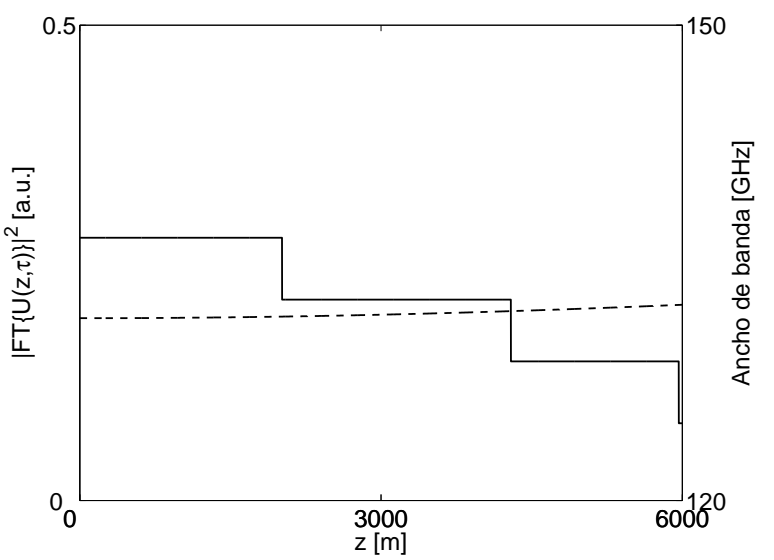

(d)

Figura 2.7: Propagación de un pulso gaussiano para $N \approx 1$. (a) Evolución del pulso en el dominio temporal. (b) Espectro del pulso propagado. (c) Intensidad (línea de trazos) y ancho temporal del pulso en función de la longitud de propagación. (d) Densidad espectral (línea de trazos) y ancho de banda.

tencia y ancho de pulso) no han sido modificados, en el dominio del tiempo y la frecuencia, respectivamente. Haciendo un análisis de la propagación para un solitón de orden $N \approx 1$, los cambios de intensidad y ancho del pulso observados en las Figs. 2.8 (c) y (d) en ambos dominios resultan inalterados con la longitud de propagación, hasta $L_{D} \approx L_{N L} \approx 6 \mathrm{~km}$ (suponiendo un caso ideal sin atenuación). De esta manera, se puede inferir que la solución 


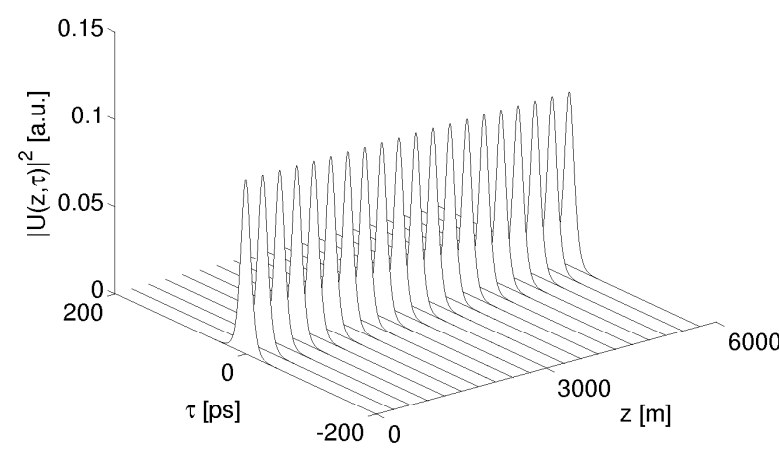

(a)

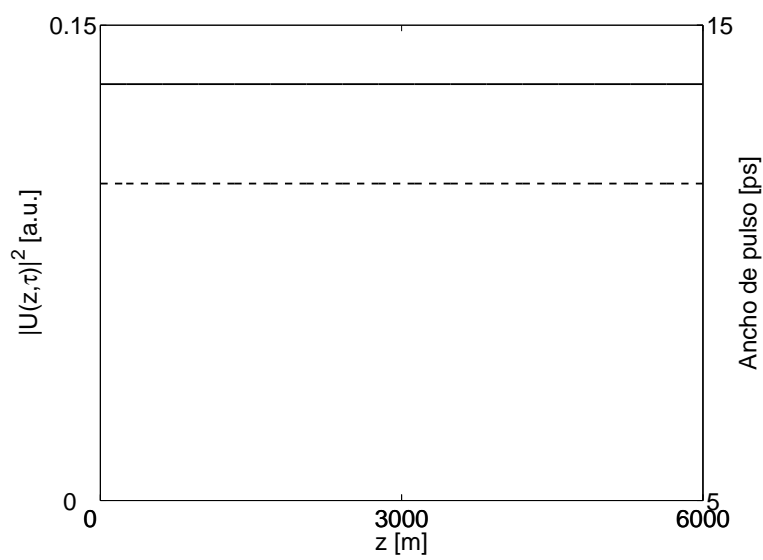

(c)

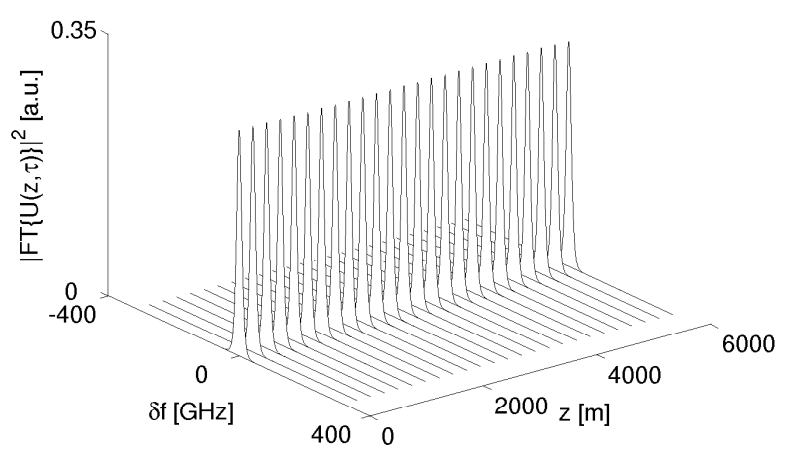

(b)

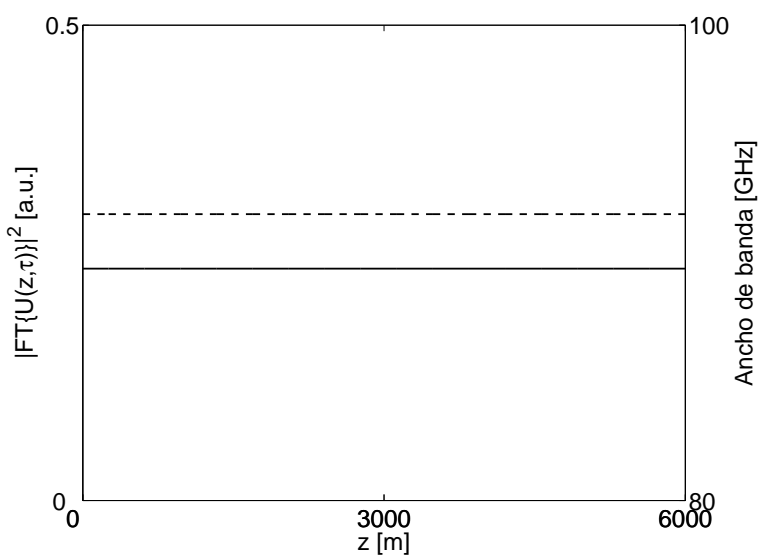

(d)

Figura 2.8: Propagación de un pulso secante hiperbólica para $N \approx 1$. (a) Evolución del pulso en el dominio temporal. (b) Espectro del pulso propagado. (c) Intensidad (línea de trazos) y ancho temporal de pulso en función de la longitud de propagación. (d) Densidad espectral (línea de trazos) y ancho de banda.

de la Ec. 2.9 resulta en un solitón, cuyo perfil es del tipo secante hiperbólica. Esta suposición se basa en que la distorsión que sufre el pulso es prácticamente nula. Este concepto será utilizado luego para explicar la interacción entre la dispersión de segundo orden y la nolinealidad en sistemas láser $[53,54]$, como se puede observar en los láseres que operan en régimen mode locking, los cuales serán tema de discusión en el Capítulo 4. 


\section{Capítulo 3}

\section{Modelado de filtros con FBGs y sus}

\section{aplicaciones}

En este capítulo se establecen las bases de la propagación de los campos eléctricos sobre una FBG de período uniforme y perfil de índice de refracción sinusoidal, como se describe en la Sección 3.1. Además se emplea el TMM para el modelado de estos dispositivos fotónicos, el cual es de gran utilidad en el análisis y síntesis de los mismos [55,56]. El cálculo de la propagación de los campos eléctricos mediante este método, descrito en el Apéndice, presenta una gran flexibilidad en el modelado y en la representación espectral de los dispositivos ya que permite trabajar en términos de la longitud de onda o la frecuencia, según se requiera. Aplicando este mismo método, se realiza un análisis de la propagación de los campos eléctricos en las FBGs de período no-uniforme, o que presentan un variación lineal o chirp de su período.

En la Sección 3.2 se muestran diversas aplicaciones de las FBGs destinadas a la generación y el procesamiento de señales ópticas coherentes. En la Sección 3.2.1 se establecen los principios de funcionamiento de las cavidades Fabry-Perot de FO, se estudia el comportamiento de estructuras fotónicas tipo DFB basadas en FBGs y se aplican dichos conocimientos al diseño de una cavidad láser formada con FBGs de período uniforme, la cual es utilizada en un láser de FO cuya emisión estimulada es pulsada y opera bajo el régimen de Q-switch. 
La Sección 3.2.2 muestra el diseño de dos filtros, un MI y un MZI, los cuales son aplicados principalmente al filtrado de canales ópticos multiplexados en longitudes de onda o WDM. Particularmente, un MI o un MZI puede ser aplicado en la modulación de las pérdidas en un sistema láser mediante el control de una condición de ajuste de la fase, la cual es dependiente de una diferencia de camino óptico definida por el ajuste de las longitudes de las ramas del interferómetro. Estos sistemas son comúnmente aplicados en láseres que operan en régimen mode locking, con el fin de obtener mayor potencia de salida y reducir el ruido de fase o jitter. En la Sección 3.2.3 se presenta la generación de líneas de retardo ópticas o true-time delay (TTD) mediante FBGs y tramos de FO estándar, para el diseño de un sistema de procesamiento óptico de formación de haz o beamforming. Dicha aplicación es de interés actual en diversas áreas de las comunicaciones en general, en diseños de sistemas de radar y en radioastronomía [57-59]. Finalmente, en la Sección 3.2.4 se presenta un método novedoso para la caracterización de pulsos cortos y ultracortos de forma completa, en intensidad y fase, para señales ópticas coherentes limitadas por transformada, el cual está basado en el uso de una FBGs de período no-uniforme (LCFBG) como elemento dispersivo.

\subsection{Modelado de FBGs}

Las FBGs son FOs, las cuales han sufrido una modificación local del índice de refracción del núcleo de las mismas, mediante la exposición a un diagrama de interferencia generado con luz UV [10,60]. El contraste de la modulación del índice de refracción, la distancia entre franjas y la longitud de la red, determinan las propiedades de la luz propagada por dichos dispositivos. El grabado de la FBG puede ser periódico, dando lugar a las redes uniformes, con chirp (variación lineal y/o no-lineal del período), entre otras. Estas propiedades permiten modificar las características espectrales de transmisión y reflexión de las FBGs. También es posible realizar un proceso de apodización durante el grabado de las redes. El mismo consiste en incrementar gradualmente la amplitud de la modulación del índice de refracción, de manera de suavizar las transiciones abruptas del mismo [61]. Las FBGs han 
demostrado ser muy versátiles en sistemas de comunicación ópticos [62], en el filtrado de canales ópticos WDM [4] y en el diseño de compensadores de la dispersión [63,64], entre otras aplicaciones. También forman parte de muchos sistemas industriales como sensores de magnitudes físicas (temperatura, presión, estiramiento, etc.) y en implementaciones como reflectores en cavidades de láseres de FO, como podremos observar en el Capítulo 4.

En el Capítulo 2 se analizó la propagación de la luz en una FO. En esta sección nos interesa definir la propagación de una FBG, con el fin de procesar señales en el dominio óptico. La teoría clásica, empleada para describir una FBG, parte de la ecuación de ondas expresada por la Ec. 2.2. Mediante una representación de los campos eléctricos de la ecuación de onda en el dominio de Fourier se obtiene

$$
\nabla^{2} \tilde{E}-\tilde{n}^{2}(z, \omega) \omega^{2} / c^{2} \tilde{E}=0
$$

donde $\tilde{E}$ es el campo eléctrico expresado en el dominio transformado y $\tilde{n}(\omega)$ es la representación del índice de refracción en función de la frecuencia angular. De una forma muy elemental, en una FBG se produce el acoplamiento de los campos electromagnéticos transmitidos y reflejados, por lo que es necesario utilizar la teoría de modos acoplados para describir la propagación de los campos electromagnéticos (propagados en una misma dirección y en sentido contrario, bajo una condición monomodo) de forma analítica. El acoplamiento de estos modos fundamentales que viajan por el núcleo de la FO se produce de forma local en las FBGs, las cuales presentan una modulación del índice de refracción generada en el proceso de fabricación y que puede ser escrita de forma analítica como

$$
\tilde{n}(z, \omega)=\bar{n}(\omega)+\Delta n_{F B G}(z)
$$

donde $\bar{n}(\omega)$ es el valor medio de índice de refracción y $\Delta n_{F B G}(z)$ es la amplitud de la modulación del índice de refracción, expresada como una función de la longitud de propagación. Para una FBG que presenta un período o espaciado constante $z=\Lambda$ (FBG uniforme) $\Delta n(z)$ puede ser escrita como una serie de Fourier, resultando 


$$
\Delta n_{F B G}(z)=\sum_{m=-\infty}^{\infty} \Delta n_{m} \exp \left(i 2 \pi m \frac{z}{\Lambda}\right) .
$$

Luego, considerando que dos ondas son propagadas en la misma dirección pero con sentido contrario (co-propagantes y contra-propagantes), estas ondas son incluidas en le Ec. 3.1 y como resultado

$$
\tilde{E}(\mathbf{r}, \omega)=F(x, y)\left[\tilde{E}_{i} e^{i \beta_{B} z}+\tilde{E}_{r} e^{-i \beta_{B} z}\right]
$$

donde $\beta_{B}=\pi / \Lambda$ es la constante de fase de primer orden de la FBG, y está relacionado a la longitud de onda de Bragg por medio de $\lambda_{B}=2 \bar{n} \Lambda$, mientras que $F(x, y)$ es la función de distribución modal para las dos ondas $E_{i}$ y $E_{r}$.

Empleando las Ecs. 3.3 y 3.4, asumiendo que los campos $\tilde{E}_{i}$ y $\tilde{E}_{r}$ verifican la condición de la SVEA para la longitud de propagación evaluada $(z=L)$, y conservando sólo los términos de ajuste de la fase, las ecuaciones de los modos acoplados en el dominio de la frecuencia resultan

$$
\begin{gathered}
\frac{\partial \tilde{E}_{i}}{\partial z}=i[\delta(\omega)+\Delta \beta] \tilde{E}_{i}+i \kappa \tilde{E}_{r} \\
-\frac{\partial \tilde{E}_{r}}{\partial z}=i[\delta(\omega)+\Delta \beta] \tilde{E}_{r}+i \kappa \tilde{E}_{i}
\end{gathered}
$$

donde $\delta(\omega)=\bar{n} / c\left(\omega-\omega_{B}\right)=\beta(\omega)-\beta_{B}$ se define como la condición de ajuste de la fase (a continuación hablamos de $\delta=\delta(\omega)$ para simplificar la notación), la cual puede ser calculada mediante la diferencia de la constante de fase evaluada a la frecuencia angular $\omega$ y $\omega_{B}$, respectivamente. Particularmente, si la intensidad de las señales propagadas dentro de una FBG son elevadas, debería introducirse un término no-lineal adicional al sistema de ecuaciones acopladas, como describe minuciosamente Agrawal en su libro [65]. El coeficiente $\kappa$ gobierna el acoplamiento de las ondas co-propagantes y contra-propagantes, y para el primer orden de la FBG se calcula mediante

$$
\kappa=\frac{k_{0} \iint_{-\infty}^{\infty} \delta n_{1} F(x, y) d x d y}{\iint_{-\infty}^{\infty} F(x, y) d x d y}
$$


donde $k_{0}=2 \pi / \lambda$ es el número de onda en el vacío. En general, $\kappa$ puede incluir cambios o variaciones (sobre un plano $x y$, transversal al eje $z$ de la FO) de $\delta n_{F B G}$ las cuales ocurren cuando el cambio de índice de refracción inducido no es uniforme sobre el área del núcleo de la FO fotosensible. Suponiendo que las FBGs son uniformes $\kappa=2 \pi \delta n_{1} / \lambda$ y, si la modulación es sinusoidal, entonces la variación del índice se escribe como $\delta n_{F B G}=\delta n_{1} \cos (2 \pi z / \Lambda)$ y $\delta n_{1}=n_{\text {eff }} / 2$, por lo que la constante de acoplamiento resulta $\kappa=\pi n_{\text {eff }} / \lambda$.

La solución general para el sistema de ecuaciones acopladas definidas por las Ecs. 3.5 y 3.6 resulta como

$$
\begin{aligned}
& \tilde{E}_{i}=E_{1} \exp (i q z)+E_{2} \exp (-i q z) \\
& \tilde{E}_{r}=F_{1} \exp (i q z)+F_{2} \exp (-i q z),
\end{aligned}
$$

donde $E_{1}, E_{2}, F_{1}$ y $F_{2}$ son constantes independientes que cumplen con las siguientes condiciones

$$
\begin{aligned}
& (q-\delta) E_{1}=\kappa F_{1} \quad(q+\delta) F_{1}=-\kappa E_{1} \\
& (q-\delta) F_{2}=\kappa E_{2} \quad(q+\delta) E_{2}=-\kappa F_{2}
\end{aligned}
$$

mientras que una solución no trivial resulta para las amplitudes de campo eléctrico definidas, resultando que se puede definir el parámetro restante como

$$
q= \pm \sqrt{\delta^{2}-\kappa^{2}}
$$

donde $q$ es dependiente de la frecuencia, como ya hemos visto. De esta manera se puede definir el coeficiente de reflexión de una FBG uniforme con modulación sinusoidal y largo $L$, como

$$
\rho(q)=\frac{E_{r}(z=0)}{E_{i}(z=0)}=\frac{i \kappa \sin (q L)}{q \cos (q L)-i \delta \sin (q L)}=|\rho| e^{i \phi_{\rho}} .
$$

El resultado de este modelo simplificado de una FBG es muy útil en la mayoría de los casos como una primera aproximación a la respuesta de una FBG real. Sin embargo, el método de 
fabricación de las mismas (método de la máscara de fase, o el de la generación del diagrama de interferencia mediante dos haces de luz UV) define el tipo de grabado (por ejemplo, si es sinusoidal o una red de Dirichlet) [10]. En este último caso, el procedimiento para encontrar una expresión analítica de la reflectividad es similar al enunciado anteriormente, aunque no es sencillo encontrar una expresión simple, como la expresada en la Ec. 3.13. De todas formas, otros tipos de grabado, inclusive los procesos de apodizado en las FBGs pueden ser evaluados adecuadamente mediante un método numérico como el TMM, desarrollado en el Apéndice. Los resultados del mismo pueden ser contrastados para una FBG uniforme con modulación sinusoidal de forma sencilla, empleando el TMM en el cálculo del sistemas de ecuaciones de modos acoplados expresado por las Ecs. 3.5 y 3.6.

\subsubsection{FBGs de período uniforme}

El diseño de una FBG uniforme se basa en la elección de algunos parámetros establecidos en el proceso de fabricación, como por ejemplo: la longitud, la variación máxima del índice de refracción y el tipo de grabado. El criterio de diseño empleado depende de cuales son las características espectrales que se desean resaltar. Como veremos a continuación, un cambio pequeño en la amplitud de la modulación del índice de refracción permite alterar significativamente la reflectividad de la FBG. Si empleamos la Ec. 3.13 para calcular el módulo y la fase del coeficiente de reflexión de dos FBGs uniformes de $\lambda_{\text {Bragg }}=1550$ $\mathrm{nm}$, largo $L \approx 2 \mathrm{~cm}$ y amplitud de modulación del índice de refracción $\delta n_{F B G 1}=23$ $\times 10^{-5}$ y $\delta n_{F B G 2}=6 \times 10^{-5}$ se pueden obtener los resultados que se muestran en las Fig. 3.1 (a) y (b), respectivamente. La característica de la fase, mostrada en la Fig. 3.1 (b), es aproximadamente lineal para valores de detuning menores que la mitad del ancho de banda de la FBG y es graficada en unidades arbitrarias para una mejor visualización. De esta manera, el retardo de grupo observado es aproximadamente constante y todas las componentes espectrales de la banda reflejada experimentan el mismo retardo. En general, el valor de $\delta n$ es controlado mediante la energía del diagrama de interferencia producido de forma local sobre la FO fotosensible y el tiempo de exposición en el proceso de fabricación 


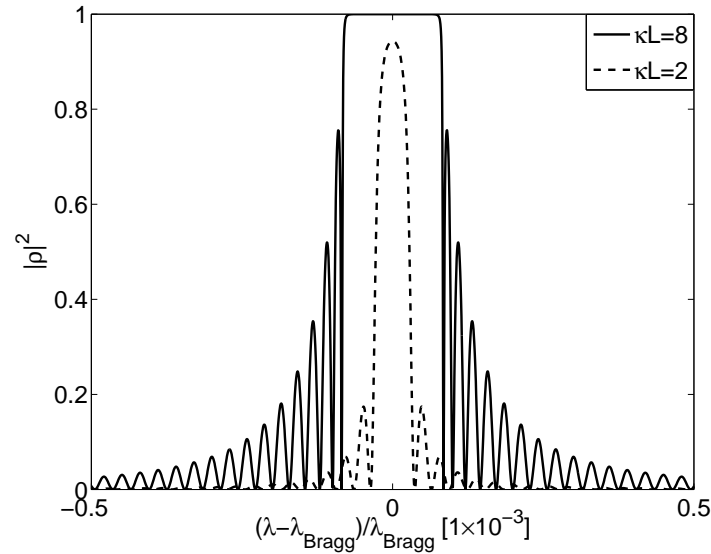

(a)

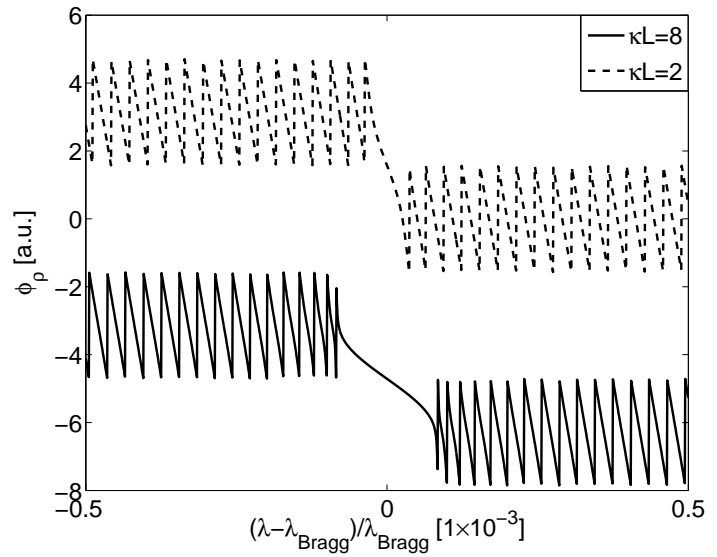

(b)

Figura 3.1: (a) Reflectividad de una FBG uniforme para $\kappa L=2$ y 8. (b) Fase del coeficiente de reflexión en unidades arbitrarias.

de las FBGs. El factor $\kappa L$, que indica el producto entre la constante de acoplamiento y el largo de la FBG, permite visualizar los cambios en el ancho de banda.

El ancho de banda medido entre los ceros de la característica de la reflectividad (módulo al cuadrado del coeficiente de reflexión) de una FBG se calcula suponiendo que $q L=i \pi$ en la Ec. 3.13, de tal forma que se anula el coeficiente de reflexión. Luego, empleando la Ec. 3.12 y la expresión de $\delta(\omega)$ anteriormente definida, se obtiene que el ancho de banda de una FBG se puede calcular por medio de

$$
\Delta \lambda_{F B G}=\frac{\lambda^{2}}{\pi n_{e f f} L} \sqrt{\kappa^{2} L^{2}+\pi^{2}}
$$

donde $\lambda$ indica la longitud de onda y $n_{\text {eff }}$ el índice de refracción efectivo. Se puede realizar una extensión del análisis anterior suponiendo que $q L=i M \pi$, donde $M$ es un número entero mayor que la unidad. De esta forma se pueden encontrar los ceros restantes de la característica de reflexión de una FBG. En general, es usual utilizar el ancho de banda medido entre los primeros ceros de dicha respuesta definido por $\Delta \lambda_{F B G}$, o bien mediante el ancho medido a mitad del máximo (FWHM) (en FBGs apodizadas, suponiendo que la atenuación de los lóbulos laterales de la respuesta de la FBG presentan una reflectividad 

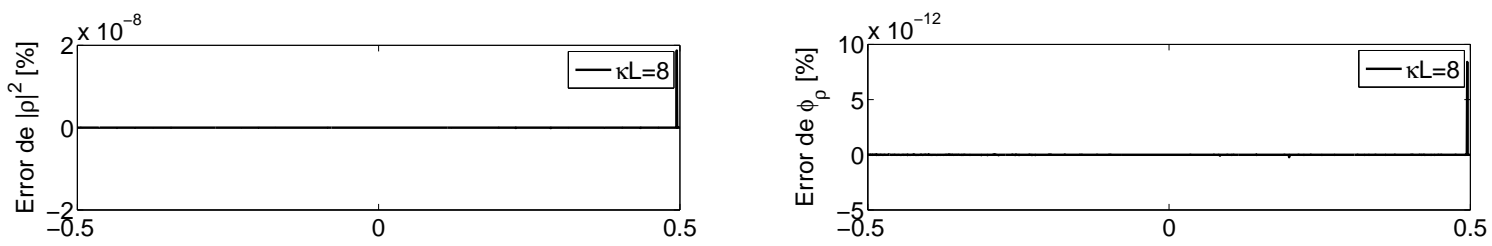

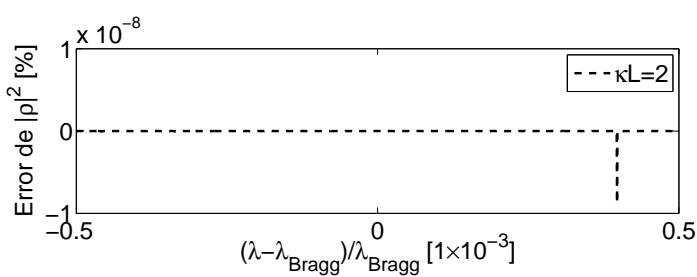

(a)

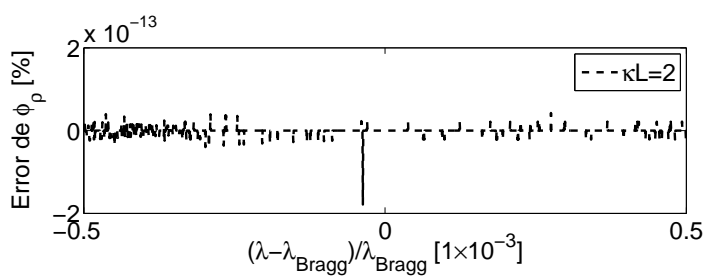

(b)

Figura 3.2: (a) Error porcentual del valor de la reflectividad empleando el TMM. (b) Error en la fase del coeficiente de reflexión.

significativamente inferior a la reflectividad del lóbulo principal), dependiendo del caso. Los resultados de la Fig. 3.1 muestran que el ancho de banda de la FBG medido a través de la Ec. 3.14 y para un valor de $\kappa L=8$ es igual a $\Delta \lambda_{F B G 1}=0,26 \mathrm{~nm}$, mientras que la FBG con $\kappa L=2$ presenta un ancho igual a $\Delta \lambda_{F B G 2}=0,11 \mathrm{~nm}$. Como podemos observar de la Ec. 3.14, el ancho de banda cambia abruptamente con la longitud física de la FBG, por lo que si se desea repetitividad en el proceso de fabricación de las FBGs la longitud de las mismas debe ser controlada de manera muy precisa.

Con el fin de estimar los errores numéricos introducidos en el cálculo de las características espectrales de las FBGs mediante el TMM, se pueden comparar los resultados obtenidos mediante este método con los resultantes de aplicar la Ec. 3.13. Los errores encontrados en la característica de la reflectividad y la fase del coeficiente de reflexión para las FBGs de la Fig. 3.1 son muy pequeños, como se observa en la Fig. 3.2. En general, los errores introducidos por el empleo del TMM están limitados a los errores de cálculo numérico, los cuales son igualmente despreciables para todos los casos analizados en este trabajo. 


\subsubsection{FBGs de período no-uniforme (LCFBG)}

Las FBGs que presentan una modulación de índice de refracción no periódica, cuando el espaciado entre las franjas $\Lambda$ no es constante con la longitud de propagación $z$, se obtiene una FBG de período no-uniforme. Un cambio en el período de la función del grabado $\left(\delta n_{F B G}(z)\right)$, puede producirse de diversas maneras: de forma lineal con la longitud de propagación [66], cambiar de forma cuadrática [67], o bien de forma aleatoria [68]. En general, las FBGs que presentan una variación lineal o chirp de su período de grabado son fabricadas exponiendo FOs fotosensibles a un diagrama de interferencia no-uniforme de luz UV. El cambio en el período de la modulación del índice de refracción de forma nouniforme se logra de diversas maneras: alterando el período de forma discreta con la posición espacial de la FO (lo que da origen a las denominadas step-chirped gratings), grabando el patrón sobre FOs con estrechamientos locales o taper, aplicando un estiramiento, presión o deformación local, entre otras [10].

La Fig. 3.3 muestra el esquema de una FBG que presenta un chirp lineal (en rigor, escalonado) del período $\Lambda$, usualmente denominadas LCFBG. Nuevamente, la propagación de los campos en estos dispositivos se basa en la teoría de los modos acoplados, de una forma que imita exactamente la función del grabado, aunque no es posible encontrar una solución analítica adecuada. Sin embargo, como la mayoría de las LCFBG se generan mediante un chirp escalonado, los métodos aplicados al cálculo son numéricos. En este sentido, el TMM es ideal para realizar el modelado de este tipo de dispositivos de FO, debido a que el grabado se realiza en secciones más pequeñas de período uniforme para un perfil de índice de refracción constante o apodizado. Una elección adecuada de las secciones de una LCFBG (cantidad y longitud de las mismas), es necesaria para aproximar adecuadamente una LCFBG de variación continua mediante una LCFBG de período discreto o escalonado. Generalmente, las secciones discretas deben expresar al menos uno o más períodos enteros de la modulación del índice de refracción de la FBG. En caso contrario, se pueden introducir errores sistemáticos debido a que se introducen saltos de fase en la función de grabado, lo cual induce algunas transiciones indeseadas en las características espectrales de estos 
dispositivos, y de las FBGs en general, como podremos observar luego en la Sección 3.2.1. Para representar adecuadamente las características espectrales de una LCFBG mediante el TMM se debe estimar el número de secciones mínimo empleando la Ec. 3.14, la cual describe el ancho de banda para una FBG uniforme. Suponiendo que cada sección de una LCFBG se expresa como una FBG uniforme de longitud $\delta l_{j}=M \Lambda_{j}$ (donde $M$ es un entero que expresa el número de períodos espaciales $\Lambda_{j}$ y $j=1 \ldots N$ es el número de sección j-ésima), que la longitud total de la LCFBG se puede expresar como

$$
L=\sum_{j=1}^{N} \delta l_{j},
$$

suponiendo que en la mayoría de los casos se cumple que $\kappa^{2} \delta l_{j}^{2} \ll \pi^{2}$ y que se verifica la condición de Bragg o igualación de la fase $(\delta(\omega)=0)$, se puede obtener la siguiente relación

$$
\frac{N}{L}=\frac{2 n_{e f f} \Delta \lambda_{L C F B G}}{\pi \lambda_{\text {Bragg }}^{2}}
$$

donde para $N=1$ secciones resulta que $\Delta \lambda_{L C F B G}=\Delta \lambda_{F B G}$, correspondiente al ancho de banda de una FBG uniforme. Sin embargo, las LCFBGs presentan un ancho de banda mayor al observado en las FBGs uniformes. Para una LCFBG de secciones discretas y con un ancho de banda total $\Delta \lambda_{L C F B G}$, el ancho de banda de cada sección $\Delta \lambda_{j}$ sólo puede ser mayor al ancho de una FBG uniforme cuya longitud es más corta, debido a la relación funcional que expresa la Ec. 3.14, aunque los anchos de banda pueden coincidir sólo si presenta una longitud apropiada. De esta manera, el ancho de banda de una LCFBG se puede estimar como

$$
\Delta \lambda_{L C F B G}=2 n_{\text {eff }}\left(\Lambda_{\text {long }}-\Lambda_{\text {short }}\right),
$$

donde $\Lambda_{\text {long }}$ y $\Lambda_{\text {short }}$ se definen como los valores del período más largo y más corto de la LCFBG, respectivamente. De todas formas, cuando no se satisface la condición $\kappa^{2} \delta l_{j}^{2} \ll \pi^{2}$, el ancho de banda resultante puede ser aproximado mediante la Ec. 3.14.

La respuesta de la fase de estos dispositivos presenta una dependencia cuadrática con la frecuencia. Por lo tanto, el retardo de grupo es lineal con la frecuencia. El cambio de la 


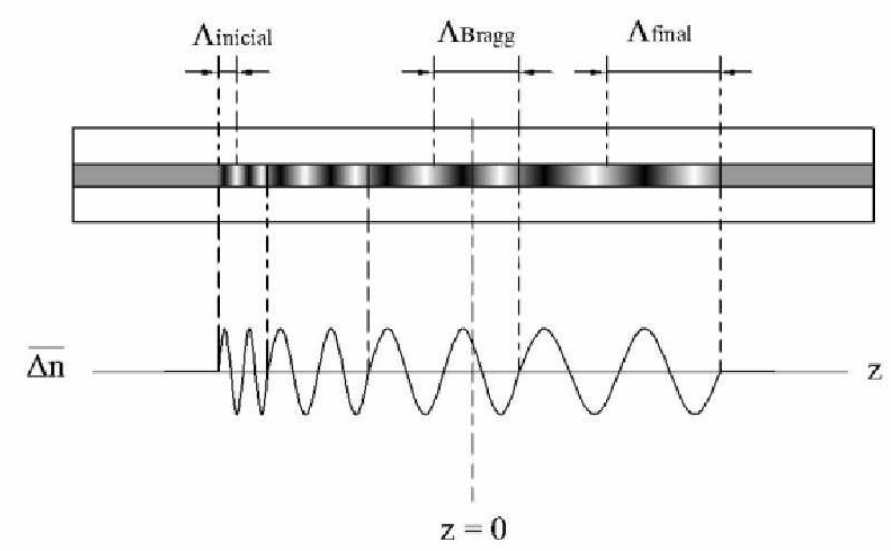

Figura 3.3: Esquema de una FBG de período no-uniforme o con chirp lineal, también denominadas LCFBG.

pendiente del retardo de grupo se logra eligiendo correctamente los parámetros de diseño tales como: la longitud de la LCFBG, la máxima variación del período o espaciado (directamente relacionado al ancho de banda) y la máxima variación de la modulación del indice de refracción que permite especificar el tipo de apodización o suavizado. Un retardo de grupo aproximadamente lineal (fase cuadrática) en la banda de paso del filtro aporta un retardo diferente para cada una de las componentes espectrales de una señal óptica reflejada por una LCFBG, permitiendo compensar eficazmente la dispersión introducida en la propagación de trenes de pulsos por una FO. La manera de compensar la CD de un enlace de comunicaciones por FO es mediante el diseño de una LCFBG, la cual tiene un valor de la pendiente del retardo de grupo igual (en módulo) a la pendiente de la CD introducida por la FO del enlace, aunque presentan un signo contrario, produciendo una dispersión nula en un ancho de banda especificado por la LCFBG. Como estos dispositivos funcionan mediante la reflexión de las señales, es común emplear un circulador óptico para redireccionar la señal compensada hacia otros puntos de un enlace. Además, el retardo de grupo presenta un ripple o variación ondulatoria pequeña en la banda de paso. El ripple puede ser incrementado para valores $\kappa L$ elevados (altos valores de reflectividad), aunque es posible diseñar LCFBGs de alta reflectividad donde los efectos del ripple son minimizados 


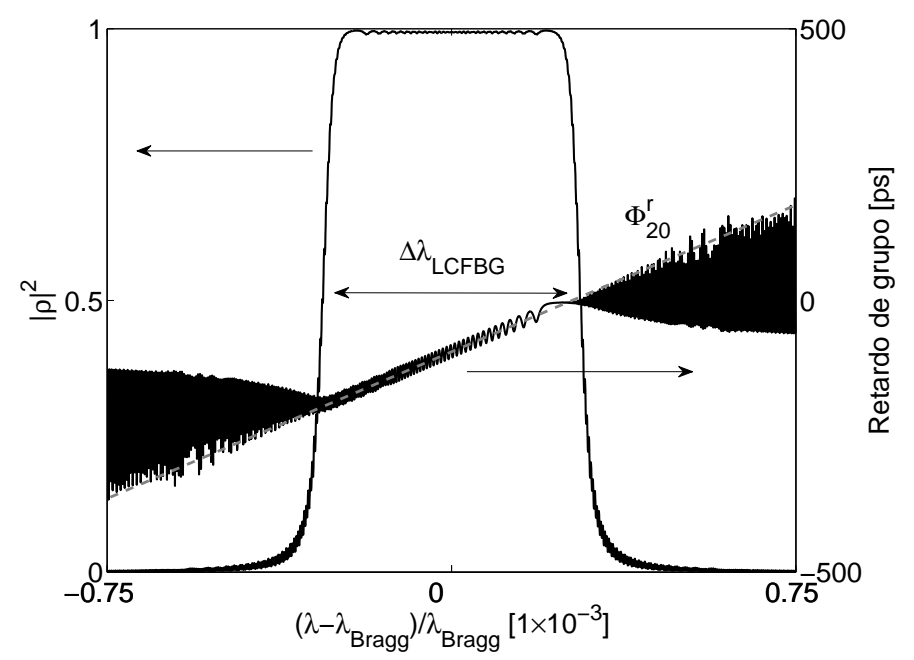

Figura 3.4: Reflectividad y retardo de grupo de una LCFBG de largo $L \approx 12 \mathrm{~cm}$, ancho de banda $\Delta \lambda_{L C F B G}=0,8 \mathrm{~nm}$, reflectividad $|\rho|^{2}=0,99$ y pendiente del retardo de grupo $\Phi_{20}^{r}=312,5 \mathrm{ps} / \mathrm{nm}$.

mediante el apodizado de la modulación del índice de refracción, por ejemplo empleando distinto tipo de ventanas en el grabado (tipo coseno elevado, tangente hiperbólica, gaussiana, etc.) $[10,60]$.

En la Fig. 3.4 se puede observar la característica espectral de reflexión de una LCFBG cuyo largo es igual a $L=12 \mathrm{~cm}$, para la cual se emplean $N=80$ secciones (mayor al mínimo impuesto $\left.N_{\min }=35\right)$ para el cálculo mediante el TMM, mientras que la máxima variación del índice de refracción es $\delta n_{\max }=1 \times 10^{-5}$ y no se ha empleado ningún proceso de apodización. Se observa que el retardo producido para cada componente espectral presenta una dependencia aproximadamente lineal con la longitud de onda, cuya pendiente de valor $\Phi_{20}^{r}$ es marcada en línea de trazos color gris. Debemos notar que los cálculos realizados mediante el TMM para este caso son en el dominio de la frecuencia y no en términos de la longitud de onda. El motivo de esta representación se basa en que las señales ópticas empleadas en la Sección 3.2.4 deben ser procesadas en el dominio transformado de Fourier. 


\subsection{Aplicaciones}

En esta sección se presentan las distintas aplicaciones con FBGs, mencionadas al comienzo de este capítulo. Las mismas están orientadas a mostrar el uso de estos dispositivos en diversos sistemas de FOs, tales como: láseres, filtros WDM, moduladores de la intensidad integrados a sistemas láser, líneas de retardo puras y elementos dispersivos que se emplean en un nuevo sistema de caracterización de señales ópticas.

\subsubsection{Cavidad Fabry-Perot de un láser que opera en régimen Q-switch}

Las cavidades del tipo FPI son dispositivos ópticos resonantes formados por dos espejos de alta reflectividad, separados uno del otro una distancia $d$ adecuada, donde el índice de refracción efectivo entre los espejos puede ser aire con $n_{e f f}=1$ (FPI Etalón), FO estándar con $n_{\text {eff }}=1,46$, o cualquier otro material transparente o de absorción pequeña para las distintas componentes espectrales propagadas en dicho medio. En la Fig. 3.5 se puede observar un esquema de un FPI. La función de transferencia de este filtro óptico queda definida por la relación entre la amplitud de campo eléctrico transmitido $E_{o}$ e incidente $E_{i}$. El campo de salida $E_{o}$ es el resultante de la interferencia de las múltiples ondas co-propagantes y contra-propagantes dentro de la cavidad. Dicha interferencia puede ser definida mediante una transferencia, en términos de un coeficiente de transmisión del dispositivo, o sea

$$
\tau(\lambda)=\frac{E_{o}}{E_{i}}=\frac{\left|\rho_{\text {mirror }}\right|^{2}-1}{1-\left|\rho_{\text {mirror }}\right|^{2} e^{-i \frac{2 \pi c t_{r}}{\lambda}}},
$$

donde $\left|\rho_{\text {mirror }}\right|^{2}$ es la reflectividad de los espejos, $t_{r}=2 d / v_{g}=F S R^{-1}$ es el tiempo que tarda un campo eléctrico en propagarse dentro de la cavidad de longitud $2 d$ (ida y vuelta), el cual está relacionado al rango espectral libre (FSR) del filtro. Como podemos observar, el FSR es inversamente proporcional a la separación entre las caras espejadas $d$. Pequeños cambios en dicha longitud pueden modificar significativamente la respuesta del filtro. Por otra parte, el valor de la reflectividad modifica el coeficiente de finura o finesse, el cual esta 


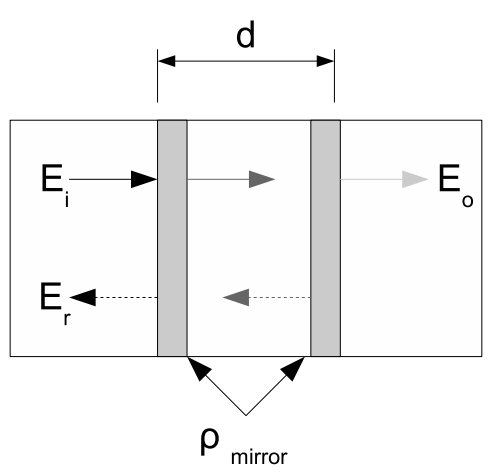

Figura 3.5: Esquema de un filtro Fabry-Perot con dos espejos de reflectividad $\left|\rho_{\text {mirror }}\right|^{2}$ separados una distancia $d$.

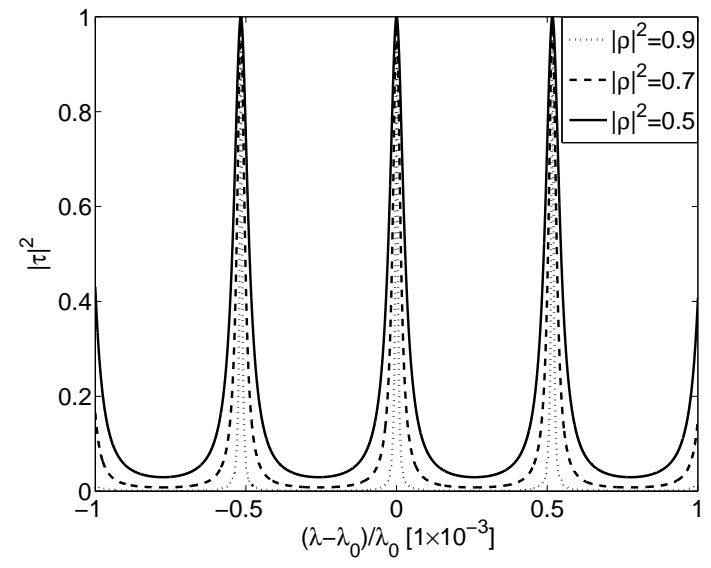

(a)

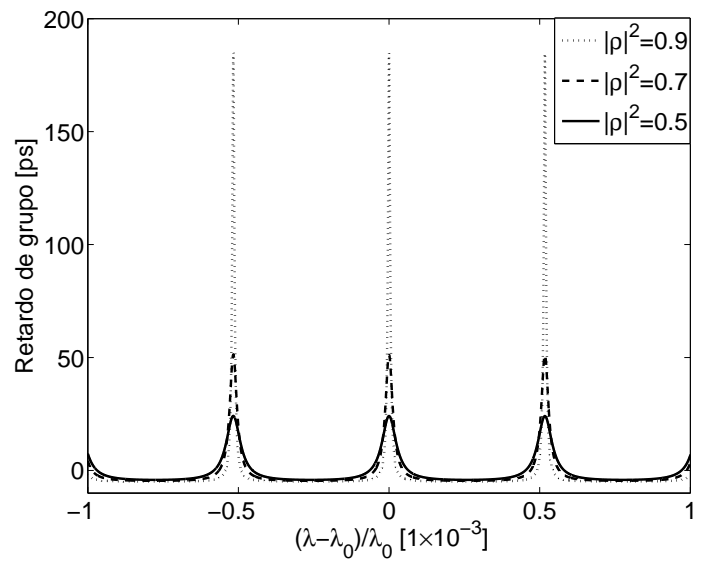

(b)

Figura 3.6: (a) Transmitancia del FPI. (b) Retardo de grupo del coeficiente de transmisión.

definido como

$$
F=\frac{\pi \sqrt{\left|\rho_{\text {mirror }}\right|^{2}}}{1-\left|\rho_{\text {mirror }}\right|^{2}}=\frac{F S R}{F W H M},
$$

donde el FWHM es medido al máximo valor de la transmitancia. El coeficiente de finura $F$ es un parámetro que indica la calidad del filtro, similar al factor de selectividad $Q$ definido en filtros electrónicos. Valores típicos rondan entre $F \approx 10-10000$, dependiendo del valor de la reflectividad de los espejos. La Fig. 3.6 (a) muestra la respuesta del FPI en función de la longitud de onda, donde $F S R=100 \mathrm{GHz}(d=1,5 \mathrm{~mm})$ y $\left|\rho_{\text {mirror }}\right|^{2}=0,9,0,7$, y 0,5 , 
mientras que la Fig. 3.6(b) muestra el retardo de grupo del dispositivo. Se puede observar que la fase presenta una característica cuadrática, en la zona espectral correspondiente a los picos de transmisión ubicados de forma periódica cada $100 \mathrm{GHz}$ (o bien 0,8 $\mathrm{nm}$ para una longitud de onda de trabajo de $1550 \mathrm{~nm}$ ). Esta propiedad es potencialmente útil en el diseño de compensadores de dispersión de tercer orden o TOD aplicados en sistemas de comunicaciones por FO.

\section{Análisis de los dispositivos de realimentación distribuida (DFB) basados en FBGs}

Para realizar un análisis particular de un cavidad del tipo FPI que es construida con FBGs en lugar de espejos, la cual es la base de diversos sistemas láser de FO, se puede estudiar el efecto que introduce una discontinuidad en el período de grabado de una FBG (tramo de FO sin grabar), también denominado como DFB [10,69]. Las discontinuidades en el grabado de las FBGs producen una alteración de las características espectrales de dichos dispositivos, induciendo uno o más picos angostos en la transmisión y/o reflexión. En general, estos picos angostos están ubicados espectralmente dentro del ancho de banda definido por las FBGs (ancho medido entre los primeros ceros). En la Fig. 3.7 se puede observar el esquema de un DFB basado en una FBG que tiene una discontinuidad en el período de grabado de longitud $\lambda / 4$. De esta manera, el defecto inducido en la FBG (tramo de FO sin grabar) es modelado por medio del TMM como una sección o capa de material cuya transferencia es definida por la siguiente matriz

$$
M_{\phi}=\left[\begin{array}{cc}
\exp \left(-i 2 \pi n_{e f f} d / \lambda\right) & 0 \\
0 & \exp \left(i 2 \pi n_{e f f} d / \lambda\right)
\end{array}\right],
$$

donde $d$ es la longitud de dicha sección y la longitud de la FBG de período uniforme es un múltiplo entero del período grabado, o sea $L=m \Lambda$ (con $m$ entero), lo que permite asegurar la validez del cálculo mediante el TMM. Por otra parte, la longitud del defecto debe ser normalizada mediante $n_{\text {eff }}$ para que la matriz $M_{\phi}$ sea consistente a un aporte de la fase igual a $\pi / 2$ para $\lambda_{\text {Bragg }}$. 


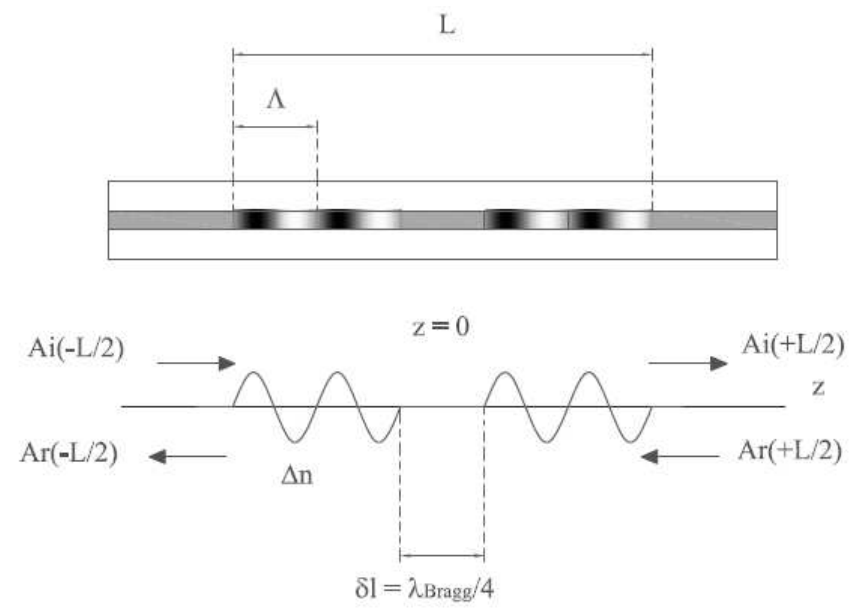

Figura 3.7: Esquema del filtro DFB basado en una FBG uniforme y una discontinuidad del período de grabado ubicada en el centro de la misma.

Las Figs. 3.8 (a) y (b) muestran la transmitancia y la fase del coeficiente de transmisión, respectivamente, de dos DFBs basados en una FBG de período uniforme. Se observa que la característica de transmisión presenta un pico abrupto dentro de la zona de atenuación de la FBG. Los parámetros de diseño del DFB son $\lambda_{\text {Bragg }}=1550 \mathrm{~nm}, L \approx 2 \mathrm{~cm} \mathrm{y} \kappa L=$ 1,2 y 2,4 , mientras que la longitud del defecto es igual a $\lambda /\left(4 n_{e f f}\right) \approx 0,27 \mu \mathrm{m}$. En la Fig. 3.8 (a) se observa que el ancho de banda del pico central es inversamente proporcional al ancho de banda de la FBG. Mediante la elección del parámetro $\kappa L$ se puede diseñar una DFB que presente un pico en la respuesta de transmisión con un valor de ancho de línea o FWHM especificado. Nuevamente, este parámetro es modificado mediante la elección de la longitud de la FBG, y/o la amplitud máxima de la modulación del índice de refracción $\delta n_{\max }$. Como se observa en la Fig. 3.8 (b), la fase tiene un salto de $\pi / 2$ radianes próximo a $\lambda_{\text {Bragg }}$, como es de esperarse para un defecto de longitud $\lambda_{B r a g g} /\left(4 n_{\text {eff }}\right)$.

Una estructura DFB basada en FBGs puede ser utilizada como un filtro de ancho de banda muy angosto, un dispositivo resonante en cavidad láser de FO, donde la coherencia es muy alta (anchos de banda muy pequeños) y en la multiplexación de señales WDM, como he mostrado en un trabajo de mi autoría [39], entre otras aplicaciones [70-72]. Los DFB se pueden diseñar de tal forma que aparecen múltiples picos de transmisión dentro del 


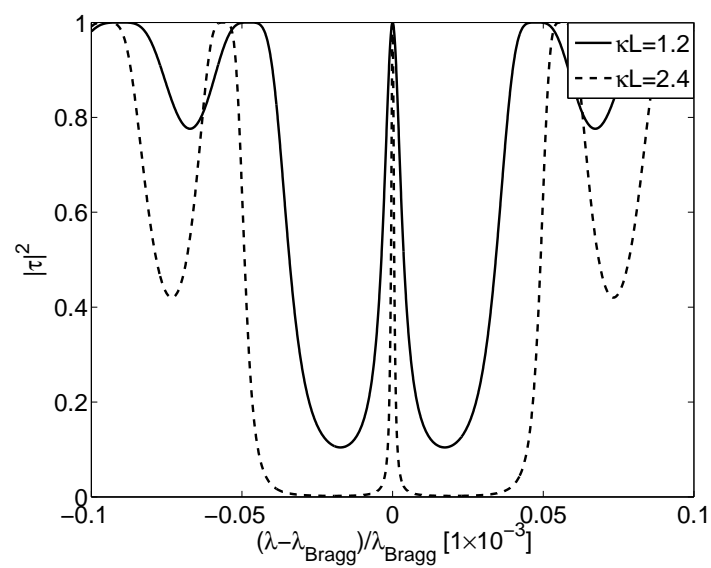

(a)

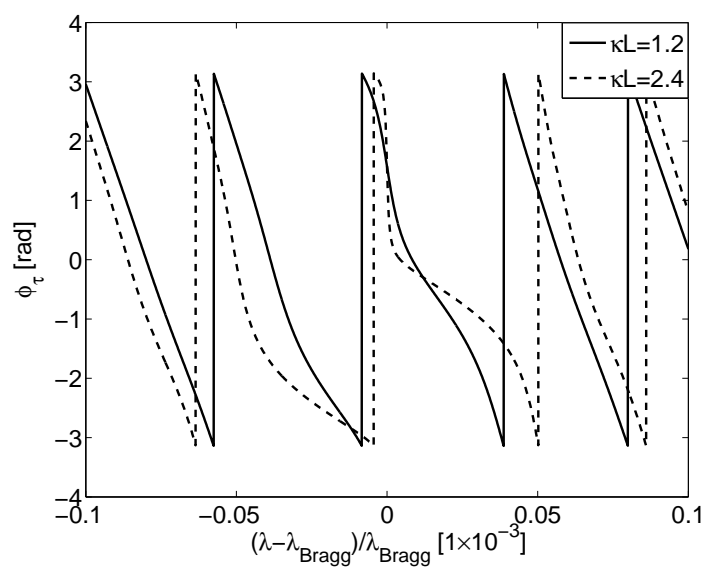

(b)

Figura 3.8: (a) Transmitancia de dos DFBs basados en FBGs. (b) Fase del coeficiente de transmisión de los filtros.

ancho de banda de la FBG (agregando múltiples discontinuidades distribuidas a lo largo de éste dispositivo). Otra forma de generar un efecto similar es modificando la longitud del defecto $d$ produciendo un cambio en la fase [69]. La Fig. 3.9 muestra la característica de transmisión de tres DFBs, los cuales se definen mediante tres valores de fase diferentes $\phi=\pi / 4, \pi / 2$ y $3 \pi / 4$. Un valor de $\phi=0$ (o múltiplos de $2 \pi$ ) no introduce ningún defecto en las características espectrales del dispositivo. De esta manera, valores de la fase expresados mediante submúltiplos de $\phi(0<\phi<\pi)$ inducen picos que se encuentran acotados espectralmente dentro del ancho de banda de la FBG. Los parámetros empleados en el diseño del dispositivo DFB son $\lambda_{\text {Bragg }}=1550 \mathrm{~nm}, L \approx 2 \mathrm{~cm} \mathrm{y} \kappa L=2,4$. Se observa que el valor del FWHM de los picos se incrementa a medida que el valor absoluto del detuning $|\Delta|=\left|\lambda-\lambda_{B r a g g}\right|$ crece, por ejemplo, para valores próximos a $\lambda_{B r a g g} \pm \Delta \lambda_{F B G} / 2\left(\Delta \lambda_{F B G}\right.$ es el ancho de banda de la FBG medido entre los primeros ceros, por medio de la Ec. 3.14). Esto se debe a que la característica compleja del dispositivo se aproxima a la condición $\kappa^{2} \delta^{2} \approx \pi^{2}$, donde la reflectividad es pequeña y el retardo de grupo presenta una respuesta no-uniforme en esa zona del espectro óptico.

Empleando todos los análisis anteriormente realizados es posible diseñar un dispositivo que presente múltiples picos en la característica de transmisión y/o reflexión, de tal forma que 


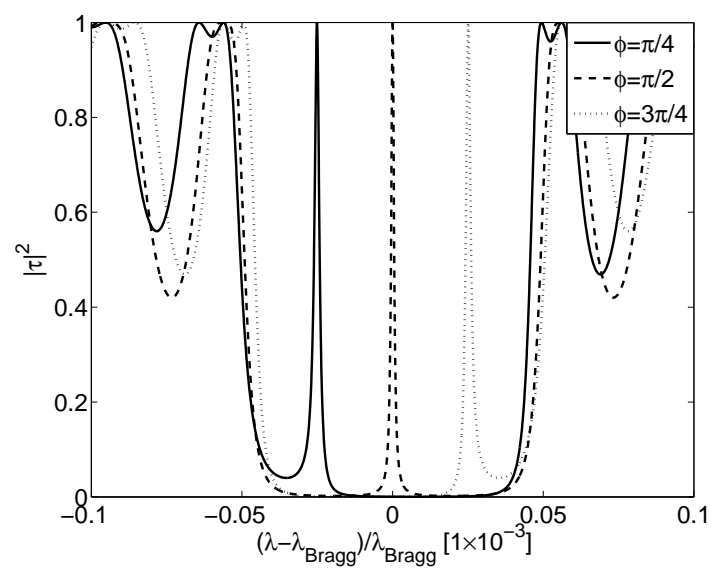

(a)

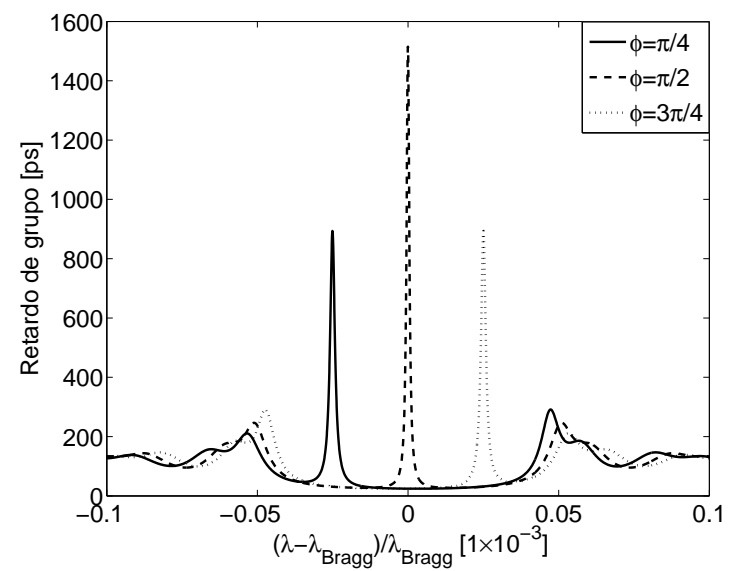

(b)

Figura 3.9: Características de transmisión de tres DFBs. Un cambio en la longitud de la discontinuidad permite modificar la posición espectral del pico de transmitancia. (a) Módulo al cuadrado del coeficiente de transmisión. (b) Retardo de grupo.

se puede imitar aproximadamente la característica del FPI formado por espejos. Los filtros FPI basados en FBGs presentan una característica que no es estrictamente periódica sino que está limitada al ancho de banda de la FBG [10,73]. El esquema de este dispositivo es idéntico al visto en la Fig. 3.7, aunque la longitud de la discontinuidad o defecto del grabado $d=m \lambda_{\text {Bragg }} /\left(4 n_{e f f}\right)$ (con $m$ entero) es varios órdenes de magnitud mayor a la observada para una estructura DFB. En estos casos, el ancho de banda total del filtro queda definido principalmente por el ancho de banda de la FBG, el cual fue definido en la Ec. 3.14, mientras que el ancho de línea de los picos inducidos en el espectro depende principalmente de la longitud del defecto. La Fig. 3.10 muestra la transmitancia y la fase de un FPI basado en FBGs cuya longitud es igual a $L \approx 0,5 \mathrm{~mm}, \delta n_{\max }=3 \times 10^{-4}$ y $12 \times 10^{-4}$ (resultando en $\kappa L \approx 1,33$ ), mientras que el defecto presenta una longitud aproximada $d \approx$ 2,5 mm. En la Fig. 3.10 (a) se puede observar que el número de picos de la respuesta de la transmisión y su ancho de banda dependen principalmente del valor de la longitud del defecto, de forma análoga a los DFBs basados en FBGs previamente analizados. De esta manera, se puede encontrar una dependencia entre el FSR y el FWHM del filtro (en la 


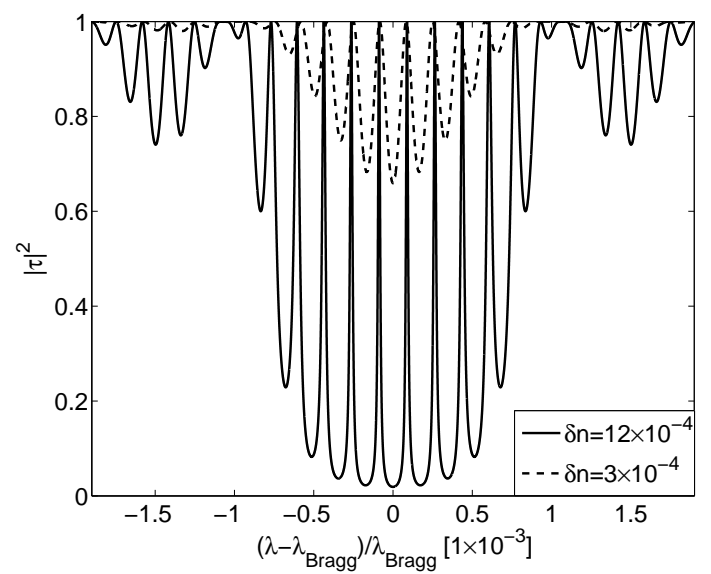

(a)

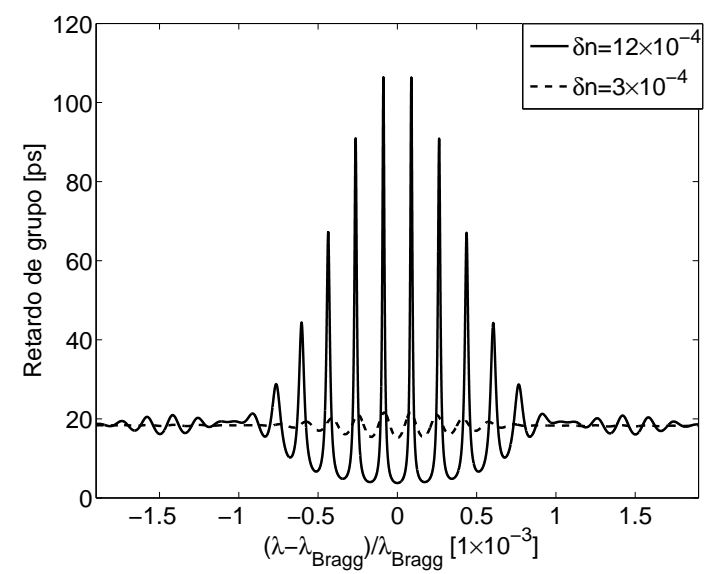

(b)

Figura 3.10: Característica de la transmisión de dos FPIs implementados con una estructura del tipo DFB basada en una FBG. (a) Transmitancia de dos FPIs diseñados con una discontinuidad de longitud $d \approx 2,5 \mathrm{~mm}$. (b) Retardo de grupo correspondiente a los coeficientes de transmisión de dichos FPIs.

zona espectral de interés, o sea $|\Delta|<\Delta \lambda_{\text {Bragg }} / 2$ ), similar a la especificada en la Ec. 3.19 pero ahora dependiente de los parámetros de diseño de la FBG. El rango espectral libre medido para este filtro es igual a $F S R=32,44 \mathrm{GHz}(d \approx 2,5 \mathrm{~mm})$, mientras que el FWHM puede disminuir rápidamente con el incremento de la longitud de las FBGs empleadas. La Fig. 3.10 (b) muestra el retardo de grupo introducido, el cual es menor, en valor absoluto, al retardo generado por el DFB mostrado anteriormente. Esto representa una ventaja de estos dispositivos frente al FPI visto anteriormente, ya que producen una distorsión menor a las señales que se propagan por el mismo.

Finalmente, se puede hacer una comparación entre el FSR de un FPI ideal y otro formado con FBGs, encontrando que para un mismo largo de cavidad (longitud efectiva para el caso de las FBGs medidas desde la zona central de las mismas), la frecuencia similar en ambos casos, como se observa en la Fig. 3.11. Sin embargo, un cambio en las longitudes físicas de las FBGs que componen el filtro puede alterar dicho característica en un rango pequeño. Por otra parte, se puede definir una longitud efectiva de la cavidad como 


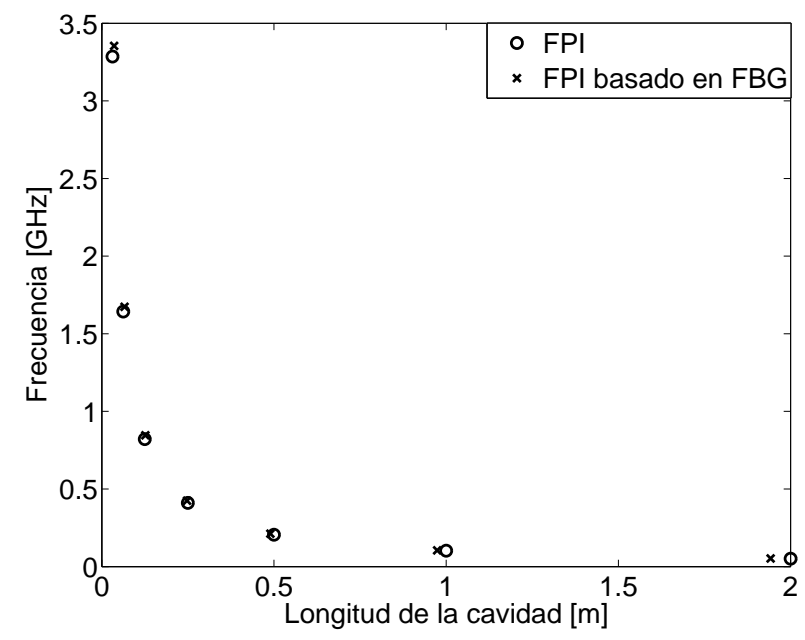

Figura 3.11: Comparación entre la respuesta de un FPI ideal y uno formado con FBGs. Las respuestas presentan valores de frecuencia similares para una longitud de cavidad $L_{e f f}$.

$$
L_{e f f}=L+d,
$$

donde $L=\left(L_{F B G_{1}}+L_{F B G_{2}}\right) / 2$ se considera como la suma de las longitudes físicas correspondientes a cada uno de los tramos de FO grabadas (mencionadas aquí como $F B G_{1}$ y $\left.F B G_{2}\right)$. De esta manera, el espaciado de las frecuencias de una cavidad FPI formada con FBGs se aproxima muy bien a las frecuencias definidas para una cavidad formada con espejos, la cual es empleada luego en el Capítulo 4. Este resultado numérico es usado ahora en el diseño de un láser de FO que funciona bajo un régimen pulsado tipo Q-switch, y será empleado luego en el Capítulo 4 en un láser de cavidades acopladas y que funciona en el régimen de operación additive-pulse mode locking (APM).

\section{Implementación de un láser que opera en régimen $Q$-switch}

Particularmente, algunos sistemas láser de FO son diseñados mediante cavidades tipo FPI basadas en FBGs, empleando FOs dopadas con tierras raras como medios de ganancia, como veremos en el Capítulo 4. En esta sección se muestra el diseño de la cavidad de un láser de FO y de emisión pulsada que opera bajo el régimen Q-switch. El mismo está 


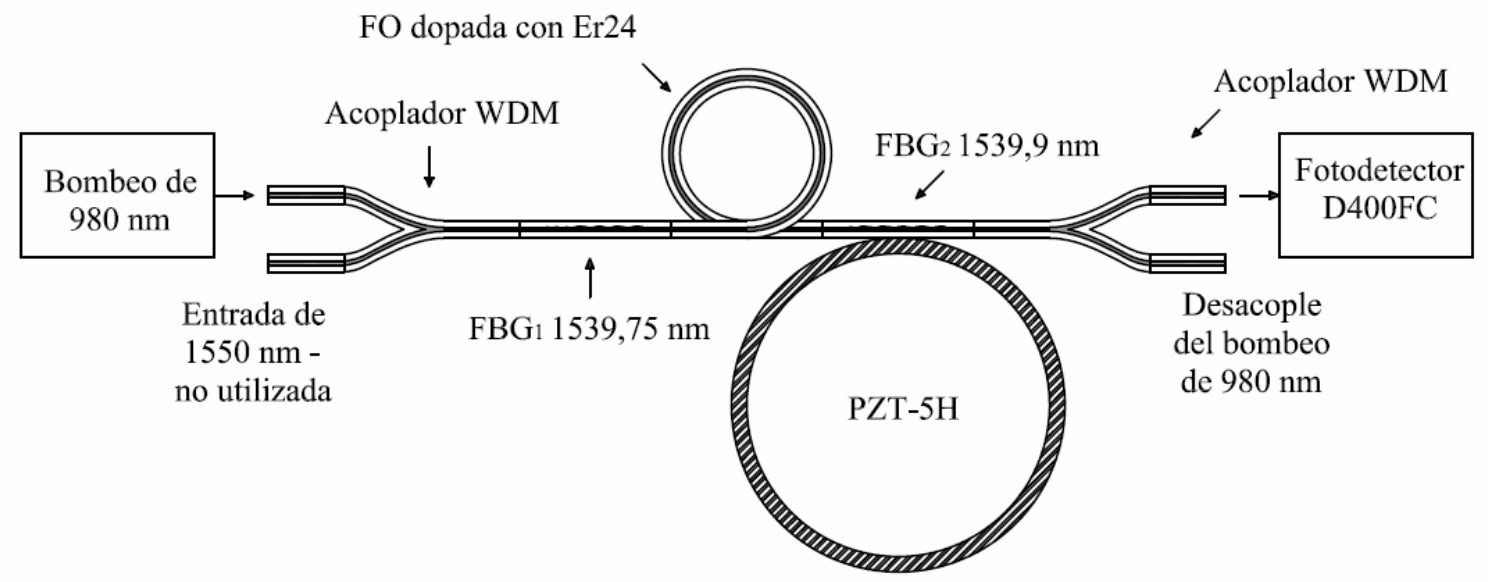

Figura 3.12: Esquema de un láser de FO que opera en régimen Q-switch basado en una cavidad del tipo FPI con FBGs y un medio de ganancia provisto por una FO dopada con $\mathrm{Er}^{3+}$. Una de las FBGs esta sujeta mecánicamente a un actuador piezoeléctrico PZT-5H de forma radial para modular las pérdidas de la cavidad.

formado por una única cavidad del tipo FPI basada en FBGs, como se puede observar en la Fig. 3.12. La implementación y caracterización de este sistema de generación de pulsos ópticos coherentes motivó su presentación en un trabajo de tesis de grado de mi autoría [40]. La cavidad está compuesta por una $\mathrm{FBG}_{1}$ fija y una $\mathrm{FBG}_{2}$ adherida a un actuador piezoeléctrico (PZT), mientras que el medio activo es implementado empleando una FO dopada con $\mathrm{Er}^{3+}$ de longitud $L=5,5 \mathrm{~m}$ (cuya concentración es igual a 790 ppm con un diámetro del núcleo igual a 4,8 um y una apertura numérica $N A=0,21$ ). En la práctica, la FO dopada con $\mathrm{Er}^{3+}$ es excitada o bombeada con un láser de semiconductor continuo de $980 \mathrm{~nm}$ y potencia máxima igual a $125 \mathrm{~mW}$, para producir la amplificación de las señales del láser, como veremos luego en el Capítulo 4. Los anchos de banda aproximados de las FBGs son iguales a $0,11 \mathrm{~nm}\left(\mathrm{FBG}_{1}\right)$ y $0,39 \mathrm{~nm}\left(\mathrm{FBG}_{2}\right)$, mientras que los valores medidos de las reflectividades son $94 \%$ y $75 \%$, respectivamente. Las características espectrales de dichas FBGs fueron simuladas mediante el TMM, con largos $L_{1}=17 \mathrm{~mm} \mathrm{y} L_{2}=4 \mathrm{~mm}$ y las amplitudes máximas del índice de refracción empleadas en el diseño de las mismas fueron igual a $\delta n_{1}=6 \times 10^{-5}$ y $\delta n_{2}=12 \times 10^{-5}$, respectivamente, con el fin de aproximarnos 


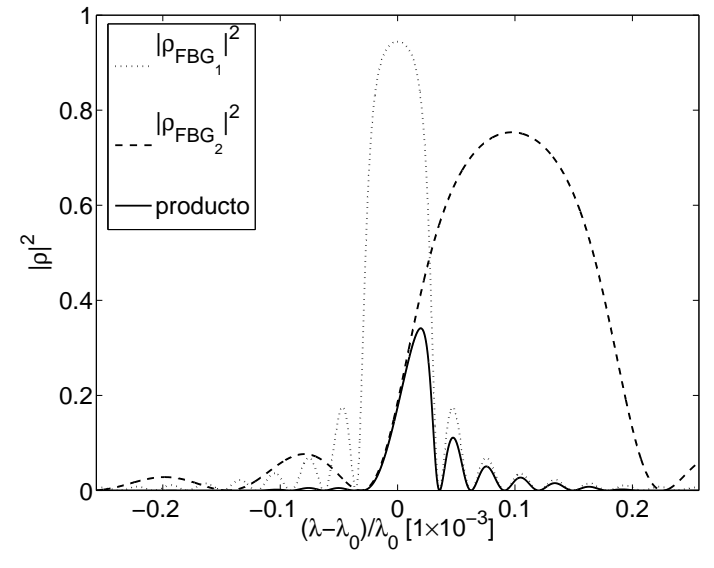

(a)

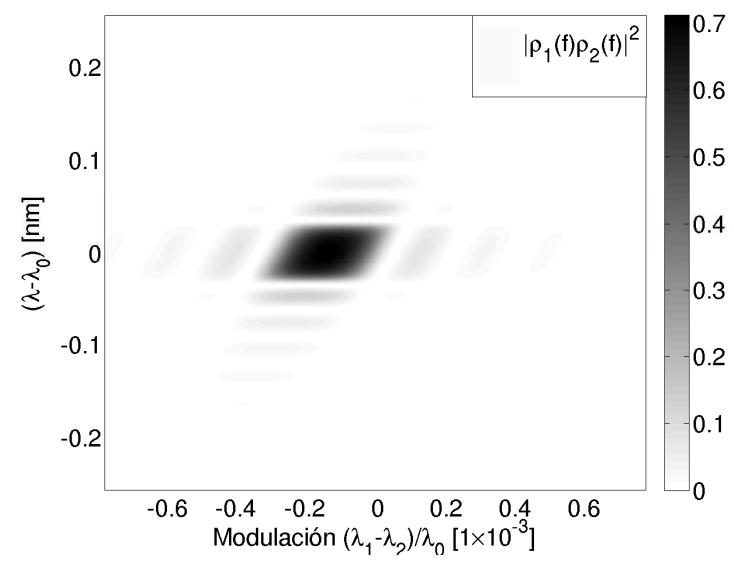

(b)

Figura 3.13: Respuesta espectral de la cavidad tipo FPI formada por dos FBGs y una FO dopada con $\mathrm{Er}^{3+}$. (a) La reflectividad de la cavidad tipo FPI es pequeña cuando la excitación del PZT es nula (no hay modulación externa), lo que induce una pérdida mayor, anulando la salida del láser. (b) Cambio de la respuesta espectral de la cavidad para diferentes valores de modulación. Se encuentran valores de reflectividad mayores para excitaciones negativas (tensión negativa sobre el PZT).

adecuadamente al comportamiento espectral real de dichos dispositivos. En este caso, no se descarta que las FBGs presenten algún tipo de apodización debido a las diferencias entre las longitudes modeladas y reales. Por otra parte, la cavidad diseñada admite frecuencias equiespaciadas aproximadamente cada $\Delta f_{\text {laser }}=c /\left(2 n_{0} L\right)=18,67 \mathrm{MHz}$ (varios órdenes de magnitud inferior al observado para una cavidad de algunos milímetros, cuyos resultados se pudieron observar en la Fig. 3.10), y correspondiente a un tiempo de round-trips de 54 ns. En un estado inicial del sistema, cuando la excitación externa del PZT es nula, no se detecta emisión láser a la salida debido a una pequeña diferencia entre las longitudes de onda de las FBGs (aproximadamente igual a 0,25 nm). Esta diferencia entre las longitudes de onda de las FBGs se traduce en un valor de reflectividad pequeño de la cavidad en su conjunto, por lo cual las pérdidas del sistema son mayores que la ganancia producida por el medio activo. 
El régimen de funcionamiento del láser se basa en la acción de actuador piezoeléctrico, el cual produce un corrimiento espectral de la característica de la $\mathrm{FBG}_{2}$ mediante un estiramiento mecánico y periódico de la misma, como se puede observar en la Fig. 3.13. En la Fig. 3.13(a) se observa el producto de las reflectividades de las FBGs para una condición donde la excitación es nula. La deformación mecánica lograda con el PZT depende principalmente de la carga mecánica adherida al mismo y del valor y tipo de tensión externa aplicada. En una caracterización realizada para tensión continua, se estima que el rango de los valores de la deformación mecánica medida se encuentra entre los 10-30 pm aproximadamente cada $100 \mathrm{~V}$ aplicados al PZT [40]. Como se puede observar en la Fig. 3.13 (b), una modulación igual a $\pm 0.2 \times 10^{-3}$ se encuentra dentro del rango especificado. Ese valor de modulación positiva produce una reflectividad de la cavidad aproximadamente nula, mientras que un valor negativo genera un incremento significativo del valor de la reflectividad de la cavidad, habilitando la emisión pulsada a la salida del láser. Los valores de deformación mecánica cambian con la tensión de excitación aplicada al PZT, observando un ciclo de histéresis, típico en este tipo de transductores electromecánicos, como se puede observar en [40]. El rango de tensiones de excitación aplicada para este caso fueron menores a $40 \mathrm{Vpp}$, por lo que la deformación calculada de forma teórica es menor a los 5 pstrain (la deformación mecánica se define como $\Delta l / l$, la cual es adimencional pero se expresa en strain) para todos los casos (menos de una décima parte de la deformación mecánica elástica y máxima de una FO, la cual se encuentra dentro de un $4 \%$ de su longitud, como Dakin [74] menciona en su libro de sensores de FO).

En resumen, cuando las respuestas de ambas FBGs se solapan en frecuencia, incrementando la reflectividad de la cavidad, se produce una disminución de las perdidas. En el mismo instante, el medio activo (que se encuentra excitado y altamente poblado) emite radiación estimulada en un tiempo corto, en la zona espectral correspondiente a la longitud de onda de las FBGs. La dinámica de este proceso puede observar en la Fig. 3.14, la cual define el régimen de operación Q-switch mediante la conmutación de las pérdidas de la cavidad (conmutación del factor $Q$ ) a través de una excitación externa aplicada al PZT.

El transductor electromecánico PZT-5H (Morgan Electro Ceramic) presenta tres frecuen- 


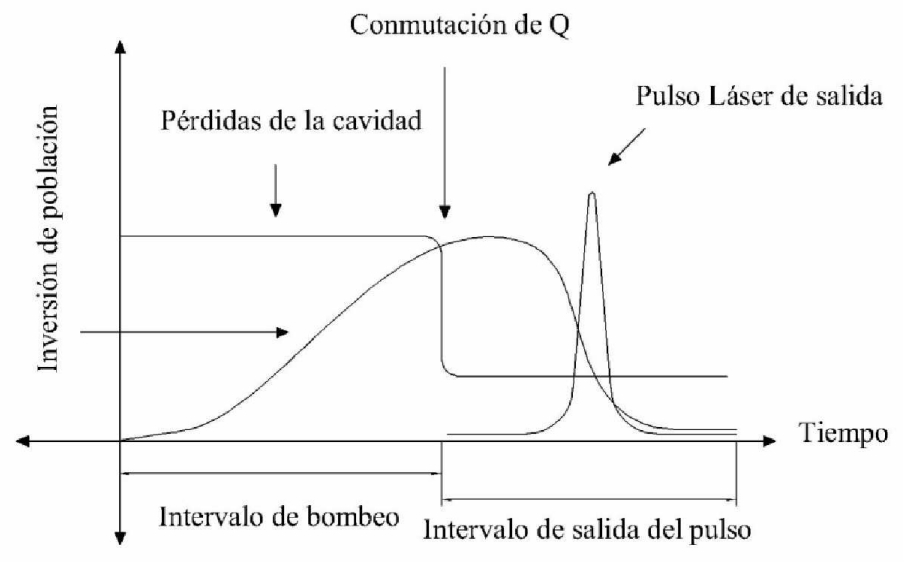

Figura 3.14: Esquema de funcionamiento del régimen Q-switch del láser de FO basado en una cavidad tipo FPI basada en FBGs. Suponiendo una condición de estado inicial excitado, al producirse una conmutación de las pérdidas se produce una inversión de la población del medio activo. La emisión láser ocurre dentro de un intervalo de tiempo en el cual la ganancia es mayor a las pérdidas.

cias de resonancia, las cuales se expresan en función de su geometría cilíndrica. Dichas frecuencias son: radial de 18,3 kHz, axial de $155 \mathrm{kHz}$ y en su espesor de alrededor de 1,57 MHz. La longitud de la cavidad activa del láser se eligió de tal forma que la frecuencia de resonancia de la misma se corresponde con un múltiplo entero de la frecuencia de resonancia radial del PZT (aproximadamente 1020 veces). De esta manera, la emisión producida es muy eficiente si se emplea la resonancia radial (cuyo valor de constante piezoeléctrica es el mayor para este PZT, de alrededor de 593 pm/V). Este diseño permite obtener una emisión pulsada coherente e intensa para valores de tensión de excitación del PZT del orden de las decenas de volts. En la Fig. 3.15 (a) se muestran las señales fotodetectada y eléctrica sobre el PZT para una excitación sinusoidal, mientras que en la Fig. 3.15 (b) se muestran las medidas de las mismas señales para un excitación del tipo onda cuadrada. Se observa que la excitación con una señal sinusoidal no es del todo eficiente pues induce una modulación en la emisión de salida, mientras que la excitación por onda cuadrada que presenta un mayor 


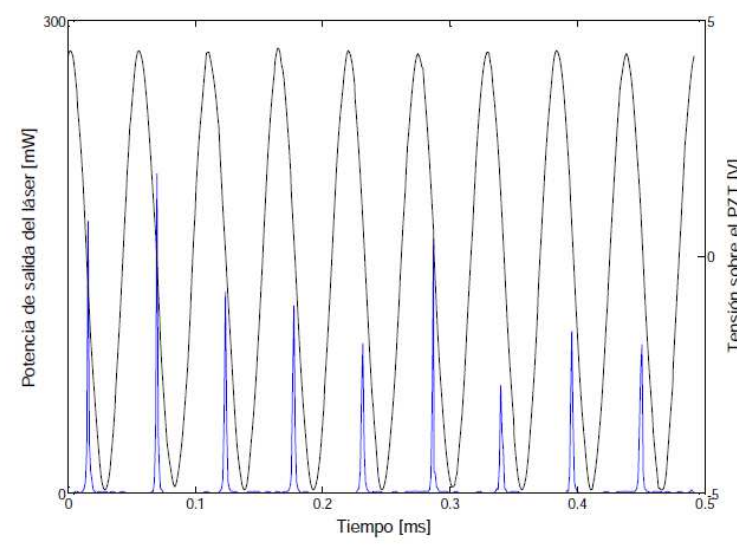

(a)

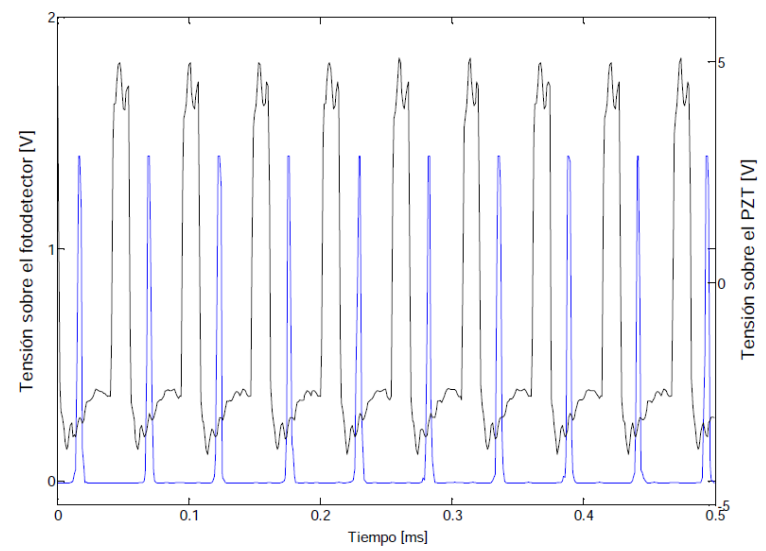

(b)

Figura 3.15: Intensidad de salida y excitación aplicada al PZT del láser esquematizado en la Fig. 3.12. (a) Excitación sinusoidal del PZT a la frecuencia de resonancia radial de 18KHz. (b) ídem para una onda cuadrada.

contenido armónico permite generar una salida óptica de intensidad uniforme. La salida óptica fotodetectada a través de la $\mathrm{FBG}_{2}$ y un acoplador WDM, mediante un detector de InGaAs de $2 \mathrm{GHz}$ de ancho de banda (EG\&G Reticon), fue registrada empleando un osciloscopio digital de $500 \mathrm{MHz}$ de ancho de banda y 2 Gsps (Hewlett-Packard HP54522A). La configuración de este láser presenta una limitación respecto a la elección de la frecuencia de los pulsos de salida. Esta frecuencia depende exclusivamente de las características electromecánicas del PZT. Por ejemplo, si deseamos obtener una frecuencia de repetición de los pulsos mayor a la observada anteriormente, debemos adherir la $\mathrm{FBG}_{2}$ al PZT en otra dirección, o bien elegir convenientemente otro tipo de transductor electromecánico. Como mencionamos, el PZT admite dos configuraciones adicionales: i) empleando la modulación mecánica axial, con una distancia menor a $10 \mathrm{~mm}$ (cuyo valor es levemente inferior a la longitud real de las FBGs); ii) utilizando el espesor del PZT, el cual sólo podría ser útil para generar una deformación local en la $\mathrm{FBG}_{2}$ (de forma similar a una estructura del tipo DFB, con un defecto de longitud variable). En el caso de que estas limitaciones mecánicas pudieran ser sorteadas, sería necesario readecuar la longitud de la cavidad del láser para producir una emisión eficiente a una tasa de repetición definida. Por último, otra 
manera de generar una emisión pulsada, empleando la misma configuración, es aplicando tensiones de excitación mayores para una frecuencia de interés. Sin embargo, esto resulta muchas veces complicado o poco eficiente, debido a las limitaciones que presenta el diseño de amplificadores electrónicos de alta tensión y frecuencia elevada [40].

\subsubsection{Sistemas de modulación de la intensidad mediante filtros interferométricos MI y MZI}

Los efectos de coherencia de señales electromagnéticas que se propagan se manifiestan por el principio de superposición que rige la interferencia de dos o más ondas [75]. Una manera de producir interferencia mediante un par de haces de luz coherentes, o más, es propagando los mismos en una misma dirección pero en sentido contrario, de modo que se establece una onda resultante estacionaria [76]. Este proceso es comúnmente generado en sistemas fotónicos que presentan una realimentación de las señales propagadas, por ejemplo, mediante el uso de un medio que refleja parcial y/o totalmente dichas señales. Como mencionamos anteriormente, en una FBG se produce un efecto de interferencia constructiva cuando la condición de ajuste de la fase es satisfecha, mientras que para otros casos se generan grandes atenuaciones en la señal resultante. Otros dispositivos fotónicos como los DFB permiten generar estructuras espectrales muy angostas en las características de transmisión y/o reflexión, mientras que el control de la longitud de un defecto en el grabado del dispositivo permite cambiar totalmente su comportamiento.

Una forma sencilla de generar interferencia utilizando dispositivos discretos de FO es mediante el uso de acopladores, los cuales permiten mezclar las señales ópticas. Algunos filtros interferométricos implementados con acopladores y FBGs (como el MI o MZI) son ampliamente utilizados en muchos sistemas de comunicaciones actuales como filtros de canales WDM, multiplexado por división de código (CDM) y multiplexación por división de tiempo (TDM), formando parte de sistemas de medición de diversas magnitudes físicas, como ya mencionamos en el Capítulo 1, o bien empleados para la modulación de señales ópticas en láseres de emisión pulsada de distinto tipo. La ventaja principal de su utilización es que 


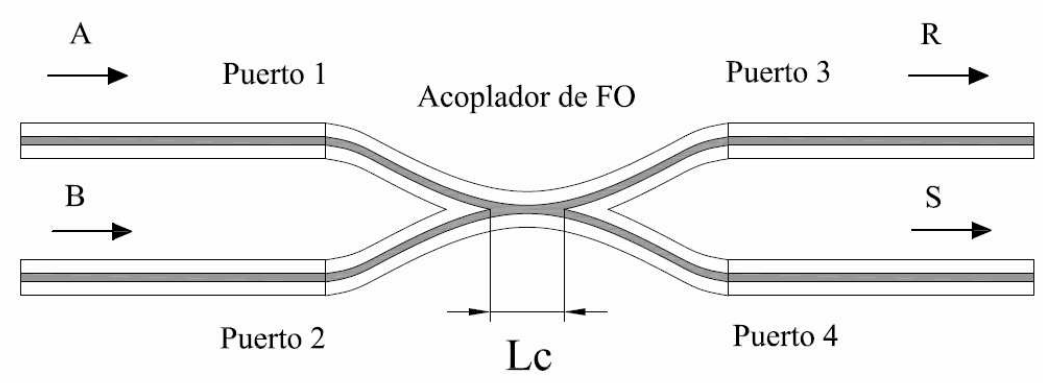

(a)

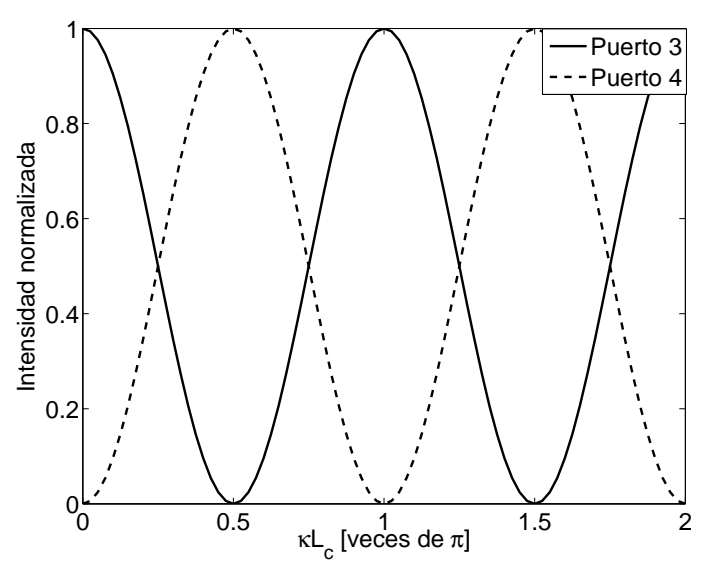

(b)

Figura 3.16: (a) Esquema de un acoplador de FO de cuatro puertos, donde $L_{c}$ es la longitud de interacción que define el coeficiente de acoplamiento del dispositivo. (b) Intensidad de salida de los puertos 3 y 4 del acoplador de $\mathrm{FO}$ en función del parámetro $\kappa L_{c}$.

son dispositivos compactos y muy sensibles frente a variaciones de la longitud de camino óptico, mientras que permiten eliminar los problemas de alineamiento introducidos en una implementación con espejos y divisores de haz (dispositivos de la óptica tradicional).

Un acoplador de FO es un dispositivo pasivo y bidireccional que permite dividir la intensidad de la luz transmitida por una FO en dos haces, mediante el esquema mostrado en la Fig. 3.16 (a). El funcionamiento es muy simple, si se inyecta luz en la rama 1 o 2, la misma es dividida y transmitida a las ramas 3 y 4 . Por otra parte, como un acoplador es un dispositivo de cuatro puertos, se puede modelar mediante una matriz de transferencia cuadrada como 


$$
\left[\begin{array}{l}
R \\
S
\end{array}\right]=\left[\begin{array}{cc}
\cos \left(\kappa L_{c}\right) & -i \sin \left(\kappa L_{c}\right) \\
-i \sin \left(\kappa L_{c}\right) & \cos \left(\kappa L_{c}\right)
\end{array}\right]\left[\begin{array}{l}
A \\
B
\end{array}\right],
$$

donde $R$ y $S$ son las amplitudes de los campos eléctricos de salida (puertos 3 y 4 ), $A$ y $B$ son las amplitudes en la entrada (puertos 1 y 2), $\kappa$ es el constante de acoplamiento que depende de los parámetros constructivos del acoplador (principalmente el solapamiento de los modos propagados por ambas FOs) y $L_{c}$ es la longitud de acoplamiento. El parámetro $\kappa L_{c}$ define el tipo de acoplamiento, 50:50, 40:60, 10:90, etc. En particular, si $\kappa L_{c}=\pi / 4$ el acoplador es 50:50 y la intensidad de entrada es dividida en partes iguales a la salida del mismo. En este caso, es también importante notar que las señales obtenidas en dichas ramas de salida se encuentran desfasadas en $\pi / 2$. En la Fig. 3.16(b) se puede observar la intensidad normalizada de los campos eléctricos de salida en los puertos 3 y 4 del acoplador, cuando se inyecta una señal de intensidad unitaria en el puerto 1 y nula en el puerto 2 . Este dispositivo es empleado muy frecuentemente en el diseño de sistemas de comunicaciones por FO basados en arquitecturas del tipo FTTx (fibra para un hogar, un edificio, entre otras). Estos sistemas de comunicación son denominados también redes ópticas pasivas o PON, las cuales emplean dispositivos ópticos pasivos como acopladores y filtros para proveer servicios de telefonía, Internet, video y audio de alta definición a múltiples usuarios, como ya se analizó en el trabajo de mi autoría [77].

\section{Implementación y caracterización espectral de un MI}

El MI es un filtro pasabanda que puede ser implementado de formas diversas, por ejemplo, mediante separadores de haz y espejos, o bien empleando un acoplador de FO y una única FBG conectada a una de las ramas de salida del mismo (puertos 3 o 4 de la Fig.3.16 (a)). Este filtro sencillo funciona de la siguiente manera: la señal inyectada al acoplador (puerto 1), que proviene de una fuente de luz coherente sintonizable en longitud de onda, es transmitida hacia los puertos de salida del acoplador. La FBG refleja una banda de longitudes de onda (como un espejo ideal de alta reflectividad y ancho espectral limitado), mientras que las demás componentes espectrales se propagan a través de esta sin atenuación 
o distorsión alguna. Las distintas componentes espectrales de la señal son reflejadas hacia los puertos de entrada del acoplador (puertos 1 y 2 ), donde se produce la interferencia. Esta configuración básica presenta algunas limitaciones: la intensidad obtenida a la salida (puerto 2) es atenuada 6dB para un acoplador del 50\%, y la interferencia producida sobre el puerto 1 coincide con la entrada del dispositivo, por lo que es necesario emplear un aislador en dicha rama para evitar que parte de la energía reflejada retorne hacia la fuente. Una solución al diseño anterior se encuentra agregando una segunda FBG en el puerto 4 del acoplador, de características idénticas a la ubicada en el puerto 3. De esta manera, se obtiene un MI doble como se muestra en la Fig. 3.17. En este caso, los campos propagados hacia las FBGs están desfasados $\pi / 2$ radianes entre sí, como se puede inferir de la Fig.3.16 (b). Ambas FBGs reflejan las componentes espectrales de una misma banda y los campos contrapropagantes viajan hacia los puertos de entrada del acoplador donde son nuevamente desfasados en $\pi / 2$ radianes, debido a la naturaleza bidireccional del mismo. De esta manera, si las longitudes de las ramas de FO del interferómetro son ajustadas adecuadamente, la señal obtenida en el puerto 1 está formada por dos componentes de igual intensidad pero desfasadas en $\Delta \theta=\pi$, por lo cual la señal se anula debido al efecto de la interferencia destructiva. En el mismo instante de tiempo, las señales reflejadas por las FBGs se encuentran en fase sobre el puerto 2 , o sea $\Delta \theta=0$, por lo que estas ondas se suman de forma constructiva. Debemos recordar, que el funcionamiento del interferómetro se basa en la coherencia de las señales inyectadas al mismo. Si las ondas que ingresan al filtro poseen una baja coherencia, la interferencia producida en las ramas 1 y 2 es débil o bien nula. En este caso particular, la intensidad reflejada por las FBGs resulta en una cuarta parte de la intensidad de entrada, cambiando el comportamiento del dispositivo y limitando el sistema a una simple suma de intensidades.

El TMM también permite describir el comportamiento del interferómetro de una forma analítica, empleando las características de transmisión y/o reflexión de los dispositivos discretos que lo componen. Suponiendo que podemos escribir el coeficiente de reflexión de una FBG en módulo y fase como $\rho=|\rho| e^{\phi}$, que el acoplador es del $50 \%\left(\kappa L_{c}=\pi / 4\right)$ y considerando que el campo a la entrada del acoplador es normalizado $(A=1$ y $B=0)$, 


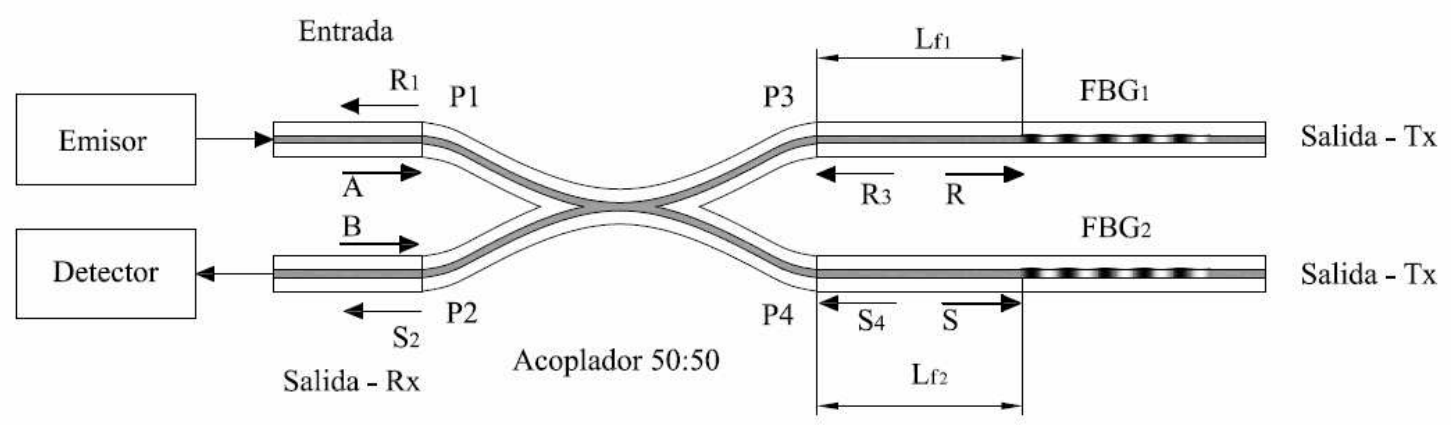

Figura 3.17: Esquema de un MI implementado con un acoplador de FO y dos FBGs.

los campos a la salida del acoplador (puertos 3 y 4) pueden ser escritos en términos de la Ec. 3.22 como

$$
\begin{gathered}
R_{3}=\left|\rho_{1}(\lambda)\right| e^{i \phi_{1}(\lambda)} \cos \left(\kappa L_{c}\right) e^{i \frac{2 \pi n_{e f f}}{\lambda} 2 L_{f_{1}}} \\
S_{4}=-i\left|\rho_{2}(\lambda)\right| e^{i \phi_{2}(\lambda)} \sin \left(\kappa L_{c}\right) e^{i \frac{2 \pi n_{e f f}}{\lambda} 2 L_{f_{2}}},
\end{gathered}
$$

donde $L_{f_{1}}$ y $L_{f_{2}}$ son las longitudes de FO entre las ramas 3 y 4 del acoplador y las FBGs, mientras que el factor 2 relacionado a estas longitudes permite expresar la propagación de ida y vuelta. Empleando estos y la Ec. 3.22 para calcular los campos de salida del filtro (ramas 1 y 2 del acoplador) se obtienen las intensidades de salida como

$$
\begin{gathered}
\left|R_{1}\right|^{2}=\frac{1}{2}\left[2 \rho_{1}^{2} \cos ^{4}\left(\kappa L_{c}\right)+2 \rho_{2}^{2} \sin ^{4}\left(\kappa L_{c}\right)-\rho_{1} \rho_{2} \sin ^{2}\left(2 \kappa L_{c}\right) \cos (\Delta \theta)\right] \\
\left|\tau_{M I}\right|^{2}=\left|S_{2}\right|^{2}=\frac{1}{4}\left[\rho_{1}^{2}+\rho_{2}^{2}+2 \rho_{1} \rho_{2} \cos (\Delta \theta)\right] \sin ^{2}\left(2 \kappa L_{c}\right) .
\end{gathered}
$$

La rama de interés (puerto 2 del acoplador) expresa por medio de $\left|\tau_{M I}(\lambda)\right|^{2}$ la transmitancia del filtro (suponiendo una entrada de intensidad unitaria), mientras que la diferencia de fase se escribe como

$$
\Delta \theta=2\left[\frac{2 \pi n_{e f f}}{\lambda}\left(L_{f_{2}}-L_{f_{1}}\right)+\frac{\left(\phi_{2}-\phi_{1}\right)}{2}\right]
$$



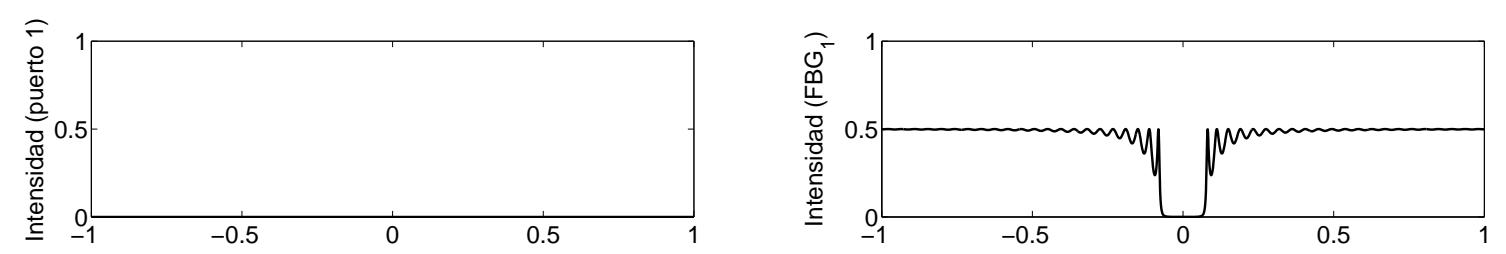

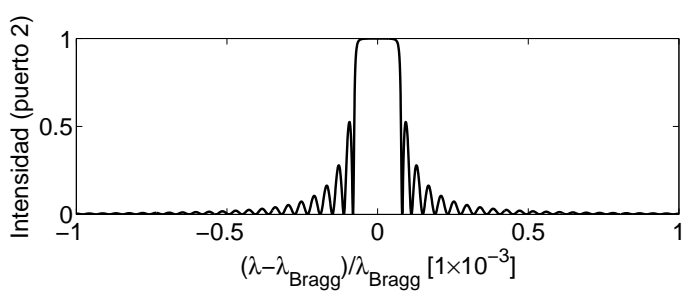

(a)

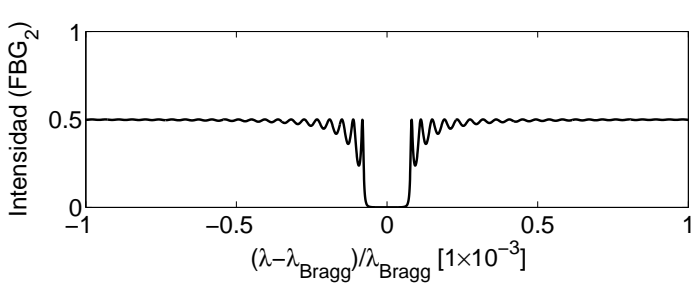

(b)

Figura 3.18: (a) Intensidad de los campos eléctricos contrapropagantes que viajan hacia los puertos 1 y 2 (salida del interferómetro). (b) Intensidad de los campos eléctricos propagados por las FBGs. Ambos resultados son mostrados en función del detuning normalizado a $\lambda_{\text {Bragg. }}$.

De esta manera, se puede inferir que, si la diferencia de fase es un múltiplo entero de $2 \pi$, toda la energía será transmitida hacia el puerto 2 (la interferencia es constructiva) y el caso contrario se presenta cuando la diferencia de fase es un múltiplo de $\pi / 2$, como será visto en el ejemplo que sigue a continuación. En la práctica, $\Delta \theta$ puede ser ajustada de forma mecánica, térmica, o empleando radiación UV sobre una de las FBGs aunque de forma permanente $[78,79]$.

Los resultados analíticos encontrados pueden ser verificados mediante un ejemplo numérico sencillo, observando las salidas del interferómetro por reflexión y transmisión de las FBGs. Suponiendo que las FBGs que forman el filtro son idénticas, con $\lambda_{\text {Bragg }}=1539 \mathrm{~nm}, L \approx 1$ $\mathrm{cm}, \delta n=20 \times 10^{-5},|\rho|^{2}=0,99$ y $\Delta \lambda_{\text {Bragg }}=0,2 \mathrm{~nm}$, el acoplador es del $50 \%$ y las longitudes $L_{f_{1}}=L_{f_{2}}$, se puede obtener la reflectividad del MI del puerto 2 del acoplador de FO, como se observa en la Fig. 3.18 (a). Si la señal de entrada es monocromática (caso ideal) y de igual intensidad para cada $\lambda$ evaluada, la interferencia producida como consecuencia de la señal propagada hacia la fuente (en el puerto 1) es destructiva, mientras que sobre el puerto 


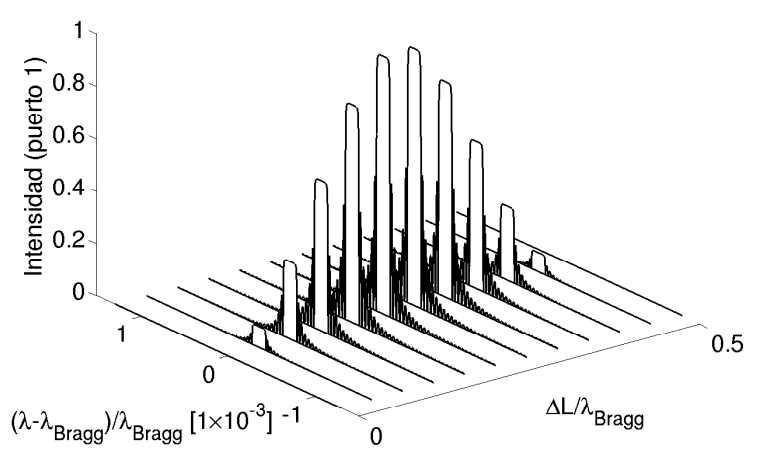

(a)

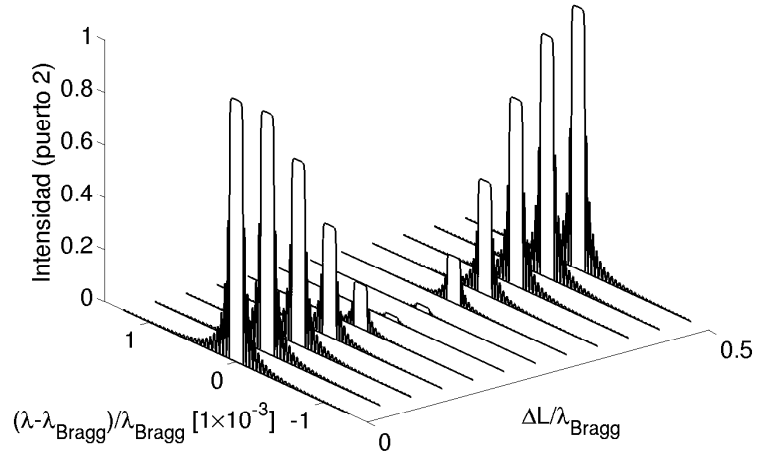

(b)

Figura 3.19: Modulación de la intensidad transmitida por el puerto 1 (a) y 2 (b) en función de la diferencia de camino normalizada a la longitud de onda de Bragg.

2 la interferencia es constructiva (las señales se suman en fase). En la Fig. 3.18 (b) se puede observar la potencia transmitida desde los puertos 3 y 4 del interferómetro, la cual es nula cuando $|\Delta|<\Delta \lambda_{\text {Bragg }} / 2$ y es igual a $1 / 2$, fuera del rango de longitudes de onda filtrado por las FBGs. Esto se debe a que el acoplador divide la señal de entrada en dos componentes de igual intensidad. Para obtener los resultados expresados en la Fig. 3.18, se supuso que la diferencia de camino óptico entre las ramas del filtro era nula y que las FBGs son idénticas en módulo y fase (resultando en la condición de ajuste, o bien, $\Delta \theta=0$ ). Estableciendo una condición de desajuste del interferómetro, o sea $\Delta L=L_{f_{1}}-L_{f_{2}} \neq 0$, se puede observar que la intensidad de salida del filtro (puerto 2 del acoplador) cambia con dicha diferencia de camino óptico de forma periódica (con período es igual a $\Delta L=\lambda_{\text {Bragg }} / 2$ ) y con una ambigüedad en la fase de valor $\pi$, como se muestra en la Fig. 3.19. Por otra parte, el comportamiento periódico del MI se establece generalmente para valores pequeños de $\Delta L$ del orden del milímetro, dentro del límite impuesto por la coherencia espacial, calculado por medio de la longitud de coherencia $l_{c}=c /\left(n_{e f f} \Delta f\right)(\Delta f$ es el ancho de banda de la señal). Cuando $\Delta L \approx l_{c}$, los efectos de interferencia se desvanecen en detrimento del funcionamiento del MI. 


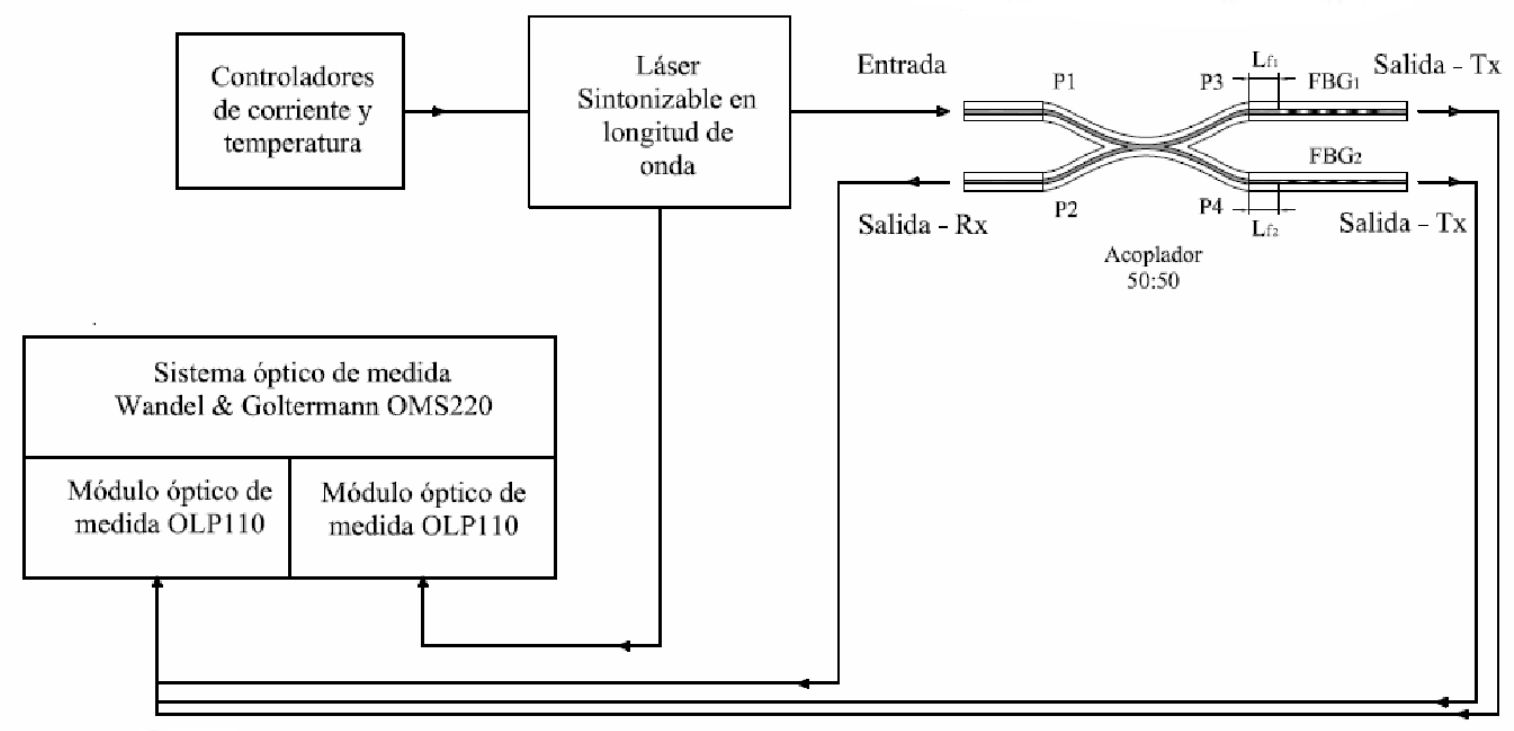

Figura 3.20: Esquema de medición del filtro MI implementado con dos FBGs.

En general, el cambio del valor de la reflectividad del MI como función de una diferencia de camino óptico puede ser empleado para modular las pérdidas de una cavidad láser (suponiendo que es posible reemplazar una de las FBGs de la cavidad por un MI), o bien, modulando la intensidad de salida de un láser mode locking, para obtener una emisión láser de pulsos ultracortos, como los producidos en [80,81]. En estos casos se busca que la emisión de salida sea nula para alguna condición de desajuste del interferómetro, la cual varía con el tiempo. Una configuración análoga sería posible haciendo uso de un modulador de fase en una de las ramas del MI, lo cual permitiría implementar otros sistemas láser con modulación externa (por ejemplo, láseres activos que operan bajo un régimen mode locking). Con estos nuevos sistemas se esperan alcanzar: emisiones láser con mayores frecuencias de repetición, mayor flexibilidad en la elección de dicha frecuencia de salida, una mejor respuesta de la fase de los pulsos generados (bajo nivel de jitter) y emisiones de mayor intensidad (respecto a sistemas láser pasivos, o bien, sin modulación externa).

En la implementación del filtro se utilizó un acoplador $50 \%$ con un error absoluto de la intensidad repartida entre las ramas del mismo menor al $5 \%$, lo que permite producir 
una interferencia adecuada [40]. Mayores desviaciones de la constante de acoplamiento del acoplador de FO se traducen en condiciones más complejas de ajuste del filtro. En el armado del mismo con dispositivos discretos se debe tener especial cuidado en la elección de las longitudes o tramos de FO (entre las ramas de acoplador de FO y las FBGs), de tal manera de minimizar las diferencias de camino óptico que puedan existir entre las ramas 3 y 4. Las características medidas de las FBGs empleadas para la construcción del filtro fueron $\lambda_{F B G_{1}}=1539,535 \mathrm{~nm}, \Delta \lambda_{F B G_{1}} \approx 0,15 \mathrm{~nm},\left|\rho_{F B G_{1}}\right|^{2}=0,8$ y $L_{F B G_{1}}=1 \mathrm{~cm}$ para la $\mathrm{FBG}_{1}$, mientras que para la $\mathrm{FBG}_{2}, \lambda_{F B G_{2}}=1539,589 \mathrm{~nm} \mathrm{y}\left|\rho_{F B G_{1}}\right|^{2}=0,64$. Los parámetros restantes son análogos a los de la $\mathrm{FBG}_{1}$. Las longitudes de los tramos de FO tipo SMF28, medidas entre el acoplador y las FBGs, fueron de 1,5 m aproximadamente, con un error absoluto en la medida de la longitud menor a $\pm 1 \mathrm{~mm}$. En la Fig. 3.20 se muestra un esquema del sistema de medida implementado para la caracterización espectral del filtro. El instrumental empleado para realizar esto fue: i) un láser sintonizable en longitud de onda marca Nano Tuning Photonetic y un controlador de corriente y temperatura para estabilizar la intensidad de salida del láser, ii) un medidor de potencia óptica marca Wandel \& Goltermann OMS-200, con dos canales conectados a dos módulos OLT-110 (rango de intensidad entre 13 y -120 dBm, con resolución de 0,01 dB). El procedimiento para realizar la medida es el siguiente: se realiza un barrido modificando la longitud de onda del láser y se mide la intensidad en cada uno de los puertos del filtro. Debemos notar en este punto que el mínimo cambio de longitud de onda para el láser sintonizable fue de aproximadamente 10 pm, por lo cual sólo se obtienen algunos puntos para definir la respuesta del filtro. Sin embargo, y pese a este inconveniente práctico, es posible estimar las características espectrales del filtro bajo una condición de ajuste, como veremos a continuación. En la Fig. 3.21 (a) se muestra la medida de la transmitancia en el puerto 2 de salida, donde se puede observar que el filtro está ajustado para reflejar una longitud de onda de 1539,6 $\mathrm{nm}$ en un ancho de banda medido de 0,14 nm (aproximadamente el ancho de banda de las FBGs empleadas), alcanzando un factor de calidad medido $Q \approx 13,995 \times 10^{3}$. En la Fig. 3.21 (b) se observa la medida de la transmitancia en los puertos 3 y 4 del filtro, donde se muestra que la intensidad en cada puerto es aproximadamente la misma, como 


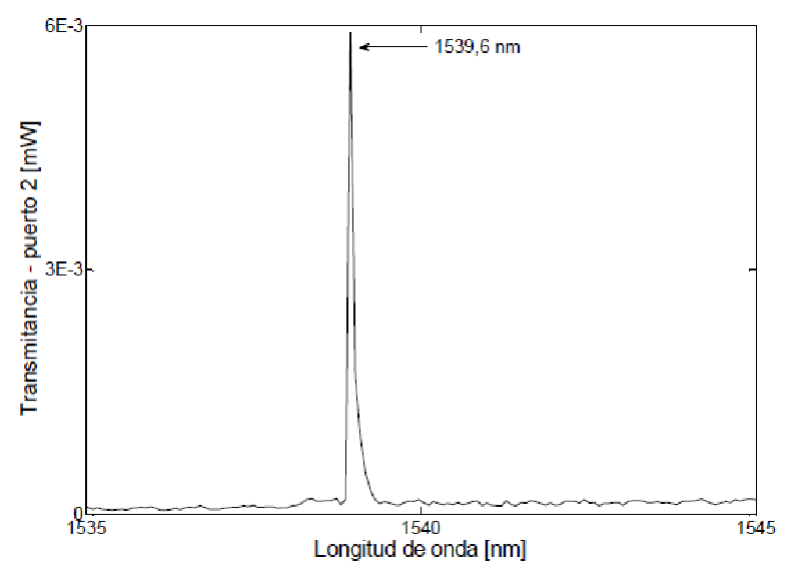

(a)

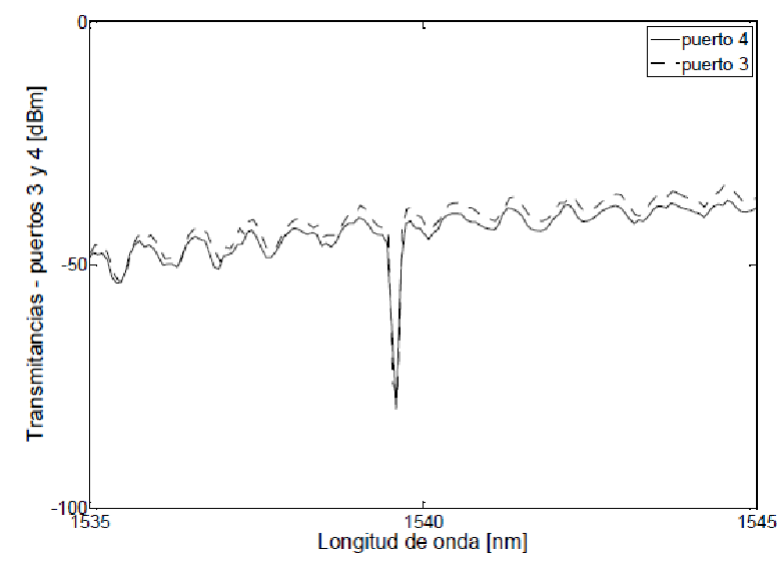

(b)

Figura 3.21: (a) Medida de la intensidad sobre el puerto 2 del MI, obtenida por reflexión de las FBGs. (b) Intensidad de los puertos 3 y 4, medidas por transmisión.

se verificó en las simulaciones previas. Las intensidades transmitidas a los puertos 3 y 4 son aproximadamente $-50 \mathrm{dBm}$ por lo que, en una condición de ajuste, la reflexión debería ser próxima a los - $25 \mathrm{dBm}$ (lo cual es similar al valor $6 \mu \mathrm{W}$, o bien - 22,2 dBm). La diferencia de 2,8 dB entre una respuesta ideal y la medida se debe a que los valores reales de las reflectividades de las FBGs no son idénticos, ni presentan un valor elevado, como el especificado en las simulaciones (reflectividad del $99 \%$ ). De esta manera, podemos asegurar que el filtro implementado se encuentra convenientemente ajustado. En general, los cambios en los valores de las reflectividades de las FBGs producen una disminución en la reflectividad total del filtro.

Experimentalmente encontramos que el ajuste del filtro fabricado con componentes discretos puede ser fácilmente alterado bajo condiciones diferentes de medida, relacionadas principalmente a cambios en la temperatura ambiente y de la polarización. Los cambios de la intensidad en las ramas del interferómetro, debido a las variaciones de la temperatura ambiente, están relacionados a la sensibilidad de las FBGs utilizadas (las cuales se encuentran en contacto directo con el aire, si una cobertura aislante adecuada). Otro factor importante para determinar un ajuste adecuado del filtro está relacionado a los efectos de la polarización, introducida en el proceso de construcción del interferómetro mediante algu- 
nos dobleces de la FO. De forma práctica, para limitar los efectos de la polarización es muy necesario fijar de forma mecánica todos los tramos de FO que componen el interferómetro para garantizar la homogeneidad de los resultados. Para obtener resultados consistentes es importante mencionar que se debió emplear el concepto de repetitividad, donde la precisión de los mismos se produce bajo condiciones idénticas de operación y sobre un intervalo de tiempo relativamente corto (durante algunos minutos y en el mismo día). De esta forma se emplea el mismo equipamiento y se desafecta los errores sistemáticos introducidos por el proceso de medida. Debemos mencionar que estos inconvenientes son subsanados mediante el empleo de tecnologías del tipo planar ligthwave circuits (PLC), basadas en la fabricación de guías de ondas acopladas y FBGs sobre un sustrato de un material transparente a la luz $[78,82,83]$.

\section{MZI aplicado a la modulación de la intensidad en sistemas láser}

Un esquema similar al anterior, pero que emplea dos acopladores de FO se muestra en la Fig. 3.22 (a), se denomina MZI y permite generar interferencia sobre una señal transmitida. El primer acoplador, divide la señal en dos haces de igual intensidad pero desfasadas en $\pi / 2$ una de la otra (como se ha mencionado anteriormente en la descripción del acoplador de FO), mientras que el segundo toma dichas señales y las encamina hacia los dos puertos de salida. Sobre uno de los puertos se produce interferencia destructiva, resultando en una señal de intensidad nula, mientras que sobre el otro las ondas concurren de forma constructiva, como se puede observar en la Fig. 3.22 (b). Debemos notar que los cambios de intensidad se expresan para pequeños desplazamientos de la fase, por lo que estos dispositivos presentan un alta sensibilidad a las variaciones de camino óptico (producidas por las ramas de FO entre ambos acopladores).

En particular, un MZI construido con acopladores y FBGs es empleado para encaminar señales WDM [10,78,79], como se puede observar en la Fig. 3.23. Esta configuración agrega un grado más de libertad a la condición de ajuste de la fase debido a la introducción de dos nuevos tramos de FO cuyas longitudes son $L_{f_{3}}$ y $L_{f_{4}}$. Realizando un análisis similar al 


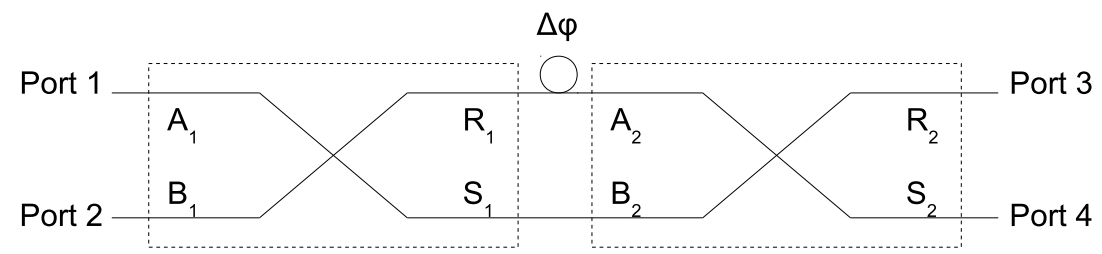

(a)

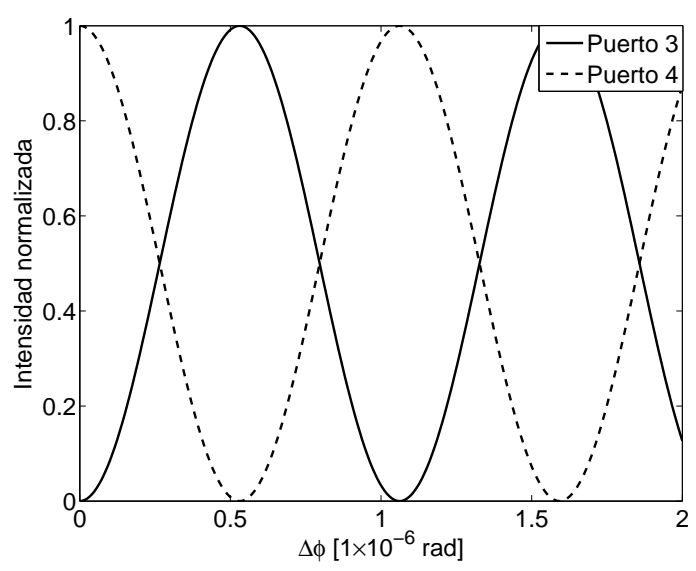

(b)

Figura 3.22: (a) Esquema de un interferómetro de Mach-Zehnder formado por dos acopladores de FO del $50 \%$. (b) Intensidades transmitidas en función del desplazamiento de la fase $\Delta \phi$ (debido a una diferencia de camino óptico entre las ramas del MZI).

anterior, e inyectando una fuente de luz coherente al puerto $P_{11}$ que se puede sintonizar en longitud de onda $\lambda$, se obtiene la salida debida a la reflexión de los campos eléctricos contrapropagantes en el puerto $P_{21}$ (de forma análoga a la respuesta de un filtro MI). Además, las componentes espectrales transmitidas a través de las FBGs se propagan hacia los puertos $P_{12}$ y $P_{22}$ del segundo acoplador de FO, resultando en las siguientes expresiones

$$
\begin{aligned}
\tau_{12}(\lambda) & =\tau_{F B G_{1}}(\lambda) e^{i \frac{2 \pi n_{e f f}}{\lambda}\left(L_{f_{1}}+L_{f_{3}}\right)} \\
\tau_{22}(\lambda) & =\tau_{F B G_{2}}(\lambda) e^{i \frac{2 \pi n_{e f f}}{\lambda}\left(L_{f_{2}}+L_{f_{4}}\right)},
\end{aligned}
$$

donde $\tau_{F B G_{1}}$ y $\tau_{F B G_{2}}$ son los coeficientes de transmisión de las FBGs, los cuales pueden ser expresados en módulo y fase, de forma análoga al coeficiente de reflexión definido en 


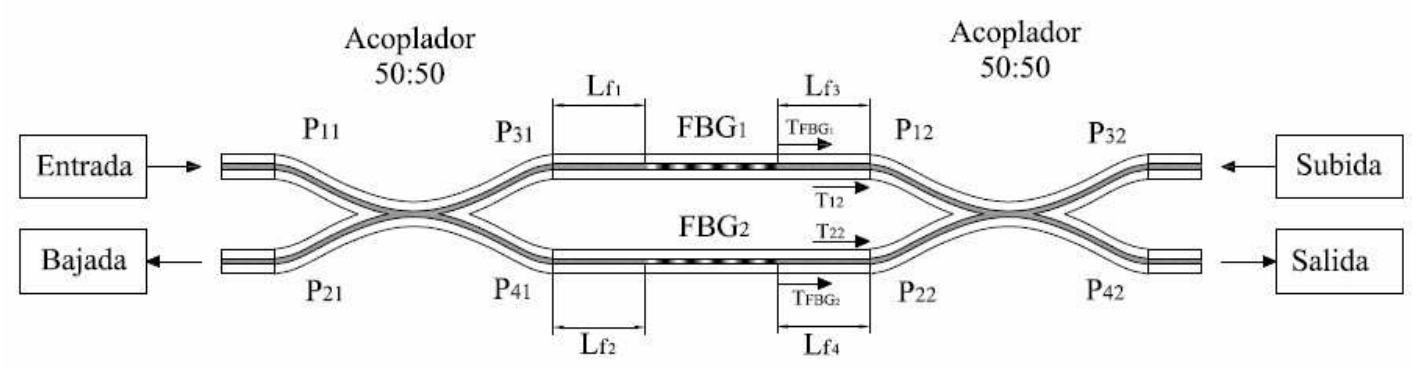

Figura 3.23: Esquema de un interferómetro de Mach-Zehnder formado por dos acopladores de FO y dos FBGs.

el MI. Las señales provenientes de ambas FBGs pasan a través del segundo acoplador y son sumadas en fase en el puerto $P_{42}$ y en contra fase sobre el puerto $P_{32}$. De esta manera, cuando el inteferómetro se encuentra ajustado, la salida resultante en el puerto $P_{32}$ es nula y toda la intensidad de las señales que se encuentran fuera del ancho de banda de las FBGs es transferida al puerto $P_{42}$. En caso contrario, cuando el filtro se encuentra desajustado, los niveles de intensidad (fuera de banda) se reparten entre ambos puertos $\left(P_{32}\right.$ y $\left.P_{42}\right)$. Considerando que la salida en $P_{32}$ es nula (condición de ajuste del filtro), es posible reinyectar una nueva señal al filtro, cuya composición espectral no debe presentar un ancho de banda mayor al filtrado por las FBGs. De forma analítica, la transmitancia en los puertos $P_{32}$ y $P_{42}$ se puede obtener mediante las Ecs. 3.28 y 3.29 , y haciendo uso de la función de transferencia del acoplador definida por la Ec. 3.22, de tal forma que

$$
\begin{array}{r}
\left|P_{32}\right|^{2}=\cos ^{2}\left(\kappa L_{c}\right) \tau_{F B G_{1}}^{2}+\sin ^{2}\left(\kappa L_{c}\right) \tau_{F B G_{2}}^{2}+ \\
+\sin \left(2 \kappa L_{c}\right) \tau_{F B G_{1}} \tau_{F B G_{2}} \sin \left(\frac{2 \pi n_{e f f}}{\lambda}\left(L_{f_{1}}+L_{f_{2}}+L_{f_{3}}+L_{f_{4}}\right)\right) \\
\left|\tau_{M Z}\right|^{2}=\left|P_{42}\right|^{2}=\cos ^{2}\left(\kappa L_{c}\right) \tau_{F B G_{2}}^{2}+\sin ^{2}\left(\kappa L_{c}\right) \tau_{F B G_{1}}^{2}+ \\
+\sin \left(2 \kappa L_{c}\right) \tau_{F B G_{1}} \tau_{F B G_{2}} \sin \left(\frac{2 \pi n_{e f f}}{\lambda}\left(L_{f_{1}}+L_{f_{2}}-\left(L_{f_{3}}+L_{f_{4}}\right)\right)\right),
\end{array}
$$

donde $\left|\tau_{M Z}\right|^{2}$ es la transmitancia del filtro, para la cual se han despreciado los factores de la fase introducida por las FBGs. Nuevamente, suponiendo que las FBGs empleadas son idénticas, la diferencia de camino óptico se traduce en una diferencia de fase que permite 

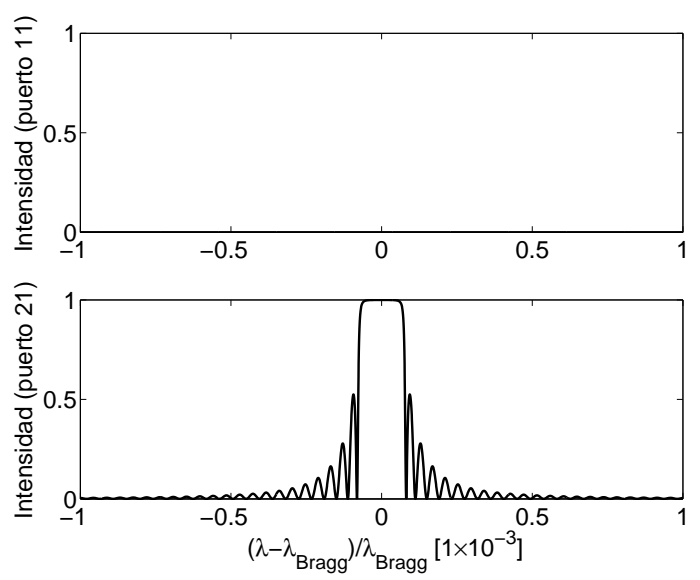

(a)
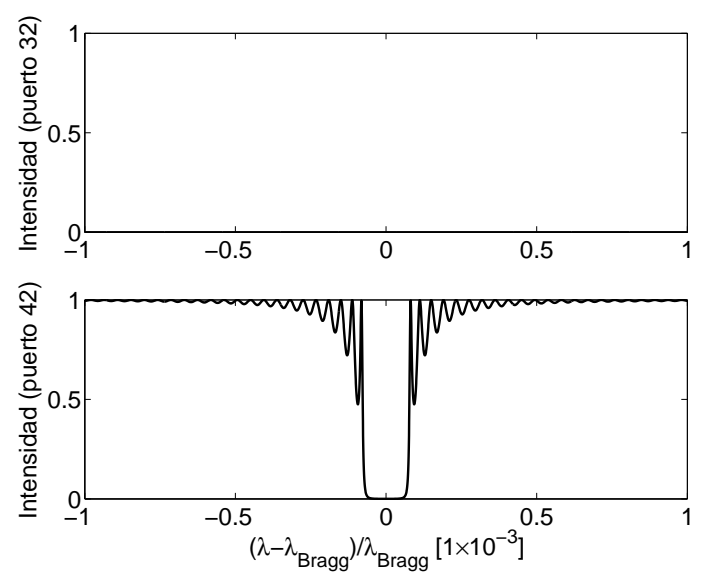

(b)

Figura 3.24: (a) Intensidad de los campos eléctricos contrapropagantes que viajan hacia los puertos 11 y 21 (salida del interferómetro por reflexión). (b) Transmitancias de salida del MZI.

seleccionar la condición de ajuste del interferómetro, o bien

$$
\Delta \theta=\left[\frac{2 \pi n_{e f f}}{\lambda}\left(L_{f_{1}}+L_{f_{2}}-\left(L_{f_{3}}+L_{f_{4}}\right)\right)\right]
$$

donde se observa que presenta un factor de escala igual a 1/2, respecto a la Ec. 3.27 encontrada para el MI. Considerando que los tramos de FO del MZI son de longitud $L_{f_{1}}=L_{f_{2}}$ e iguales a $L_{f_{3}}=L_{f_{4}}$ de tal manera que $\Delta \theta=0$, y las FBGs son idénticas a las empleadas para el MI, se pueden calcular las intensidades de salida del MZI. La Fig. 3.24 (a) muestra la transmitancia en los puertos $P_{11}$ y $P_{21}$ del filtro, las cuales son idénticas a las vistas para el MI, mientras que en la Fig. 3.24 (b) se observa la transmitancia en los puertos $P_{32}$ y $P_{42}$ de salida del MZI. La intensidad en el puerto $P_{32}$ es nula y presenta un valor unitario en $P_{42}$, salvo para el rango de longitudes de onda filtradas por las FBGs. Realizando un análisis similar al del MI y suponiendo que un desajuste en la fase es producido por un cambio de la longitud $L_{f_{3}}$, en la Fig. 3.25 se puede observar el cambio de la intensidad propagada hacia los puertos de salida para diferentes valores de $\Delta L=L_{f_{4}}-L_{f_{3}}$. Se observa que la ambigüedad de la fase es el doble que en el caso del MI, esto se debe a 


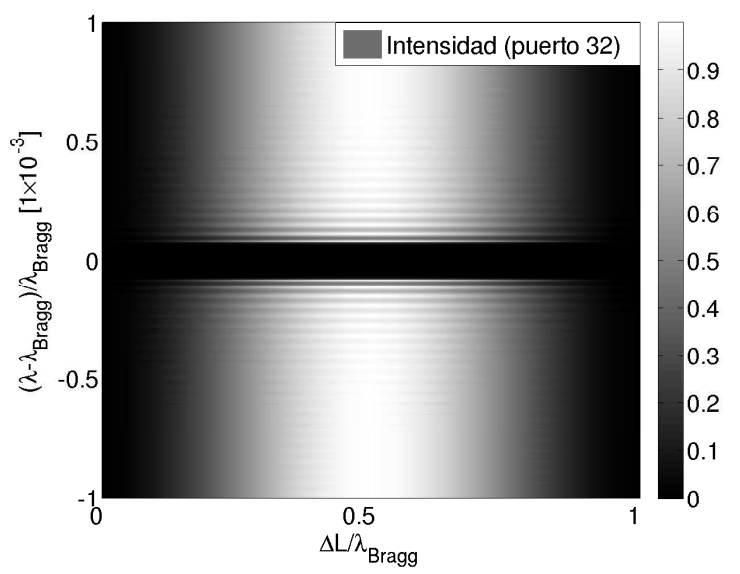

(a)

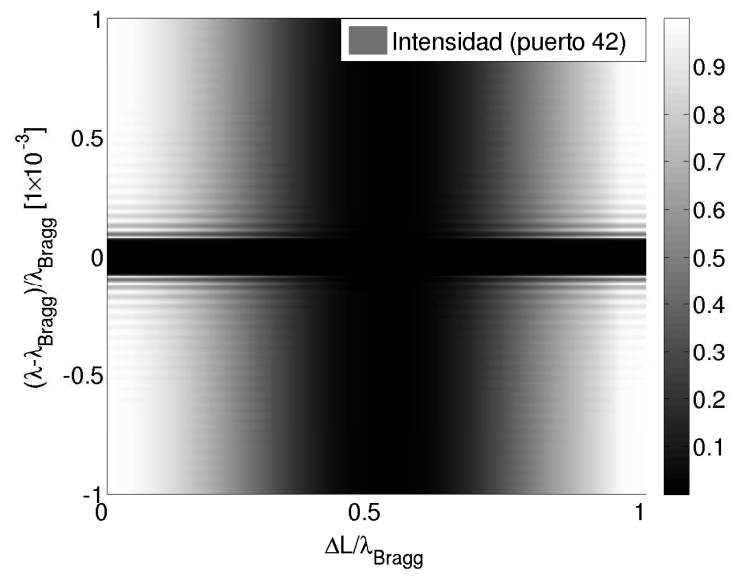

(b)

Figura 3.25: Intensidad de los campos eléctricos propagados en función del desajuste de fase $\Delta \theta$. (a) Respuesta del puerto de "subida" del interferómetro en función del desajuste. (b) Transmitancia de salida para diferentes condiciones de ajuste.

que $\Delta \theta_{M I}=2 \Delta \theta_{M Z}$, mientras que el MZI responde de la misma manera para los valores de longitudes de onda filtrados por las FBGs. Sin embargo, el valor de la intensidad para longitudes de onda que cumplen con la condición $|\Delta|>\Delta \lambda_{\text {Bragg }} / 2$ es alterado por la acción del desajuste en la fase $\Delta \theta$, el cual funciona en la forma de una interferencia destructiva para $P_{32}$ y al mismo instante de tiempo lo hace en forma constructiva sobre el puerto de salida $P_{42}$.

Este tipo de dispositivos son muy utilizados como moduladores de intensidad, fabricados con guías de onda ópticas sobre sustratos de $\mathrm{LiNbO}_{3}$, los cuales son empleados en transmisores para modular láseres de distinto tipo como: CW, DFB y de emisión pulsada en régimen mode locking, entre otros [42,84-86]. La ventaja de un MZI frente a un MI, en la implementación de un sistema de modulación para un láser de FO, es que permite modificar fácilmente las pérdidas mediante un cambio de la intensidad de las señales propagadas por el mismo. Esta característica ha dado origen a diversas publicaciones y patentes relacionadas con láseres de FO que operan en régimen mode locking [29,41,87]. Estos sistemas, generalmente formados con cavidades tipo anillo, son muy eficientes y permiten generar 


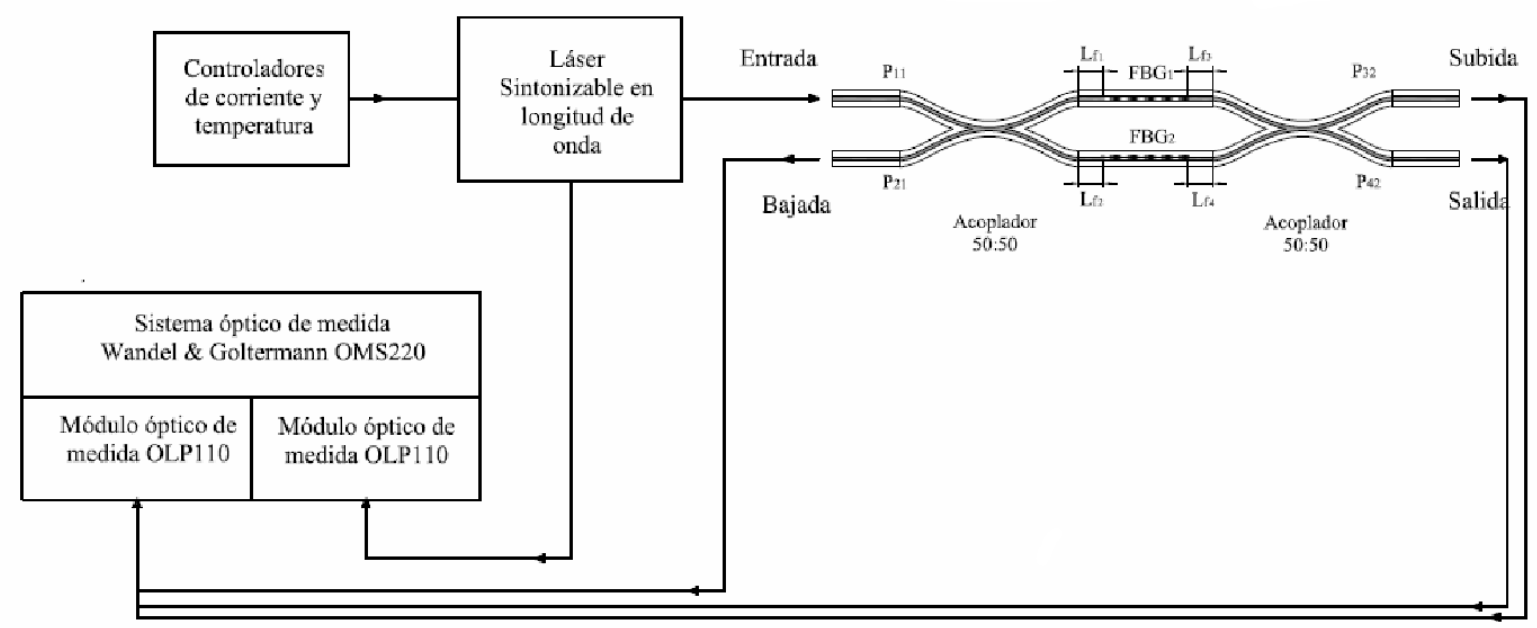

Figura 3.26: Esquema de medición del MZI implementado con dos acopladores de FO y dos FBGs.

trenes de pulsos de muy alta frecuencia y bajos niveles de jitter.

La implementación real de un MZI se realizó partiendo de la extensión del MI anteriormente descrito y el uso de un acoplador de FO adicional, como presenté oportunamente en mi trabajo [40]. El acoplador introducido presenta características similares a las ya mencionadas, mientras que las longitudes de los tramos de FO tipo SMF28 entre las FBGs y el segundo acoplador son del orden de los 1,5 m, con un error en la medición de la longitud del orden de $\pm 2 \mathrm{~mm}$. Sin embargo, y como podremos observar en la caracterización espectral que se muestra a continuación, el ajuste del filtro (para establecer la condición de ajuste de la fase donde la intensidad de la interferencia es máxima) resulta más complejo que para el caso del MI. Esto se debe principalmente a que el sistema es más sensible frente a un cambio en la diferencia de camino óptico entre las ramas del interferómetro, como hemos podido comprobar mediante la Ec. 3.32 y en las simulaciones mostradas en la Fig. 3.25. El esquema de medida empleado para la caracterización espectral del MZI es análogo al empleado anteriormente para el MI y se muestra en la Fig. 3.26. La Fig. 3.27 (a) muestra las medidas por reflexión de las FBGs, utilizadas en la implementación del MI y MZI (medidas de forma separada con un único acoplador de FO). Se observa que las 


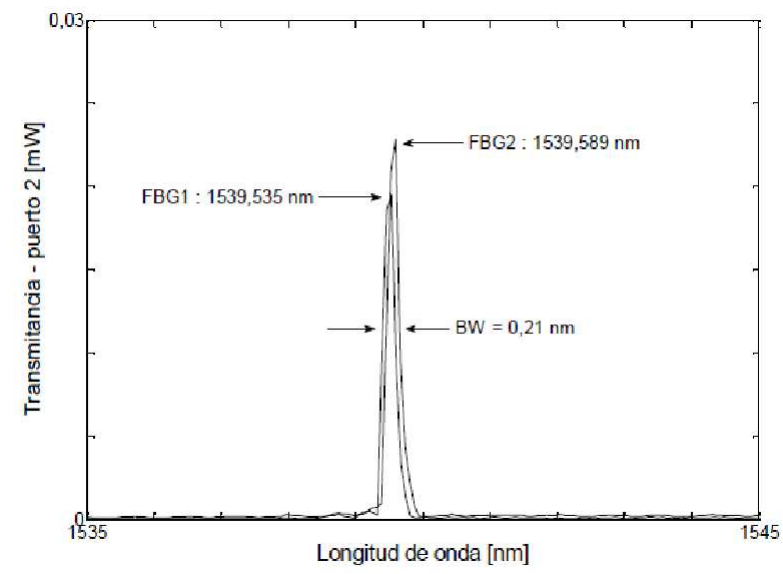

(a)

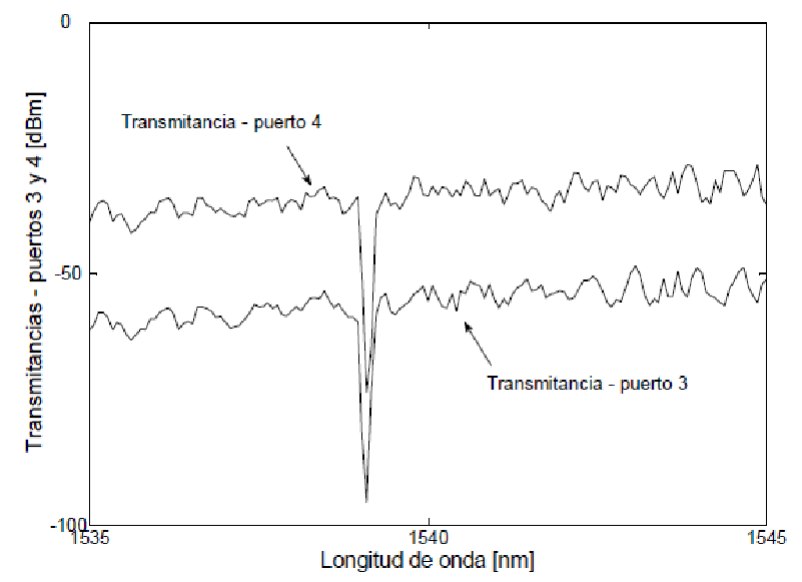

(c)

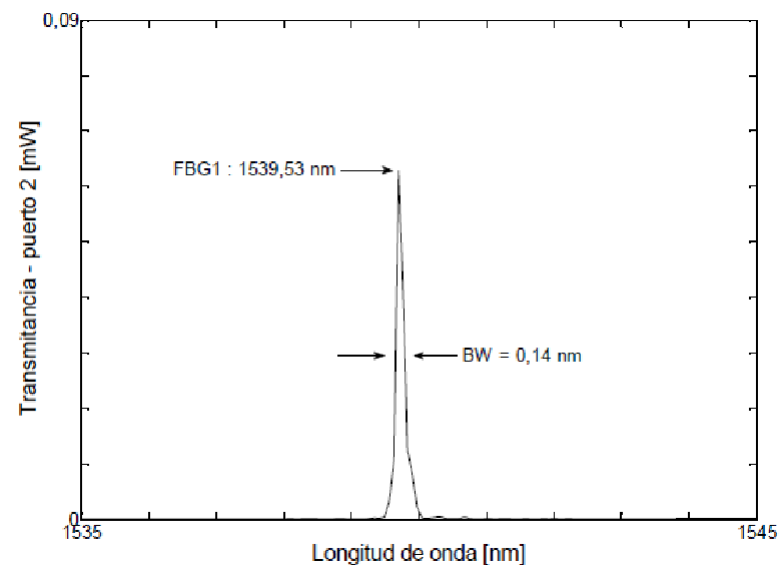

(b)

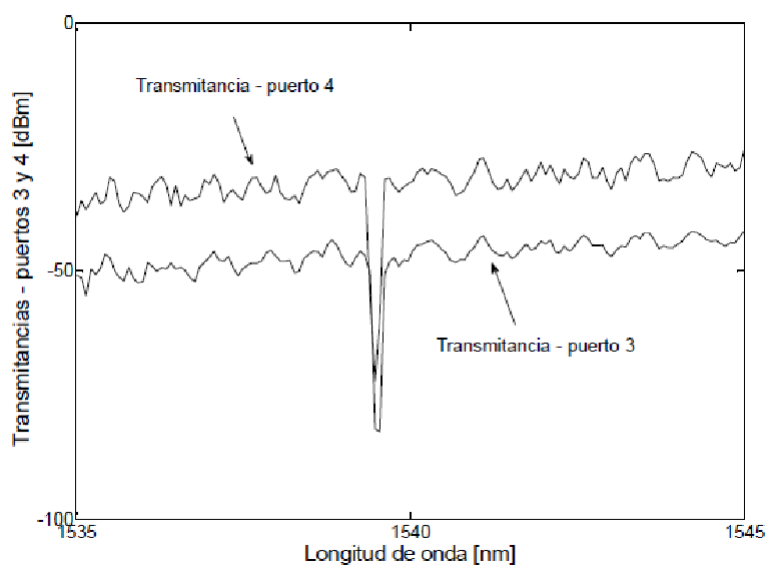

(d)

Figura 3.27: (a) Intensidad reflejada por cada una de las FBGs empleadas en la implementación del MI y MZI (medidas de forma separada). (b) Medida de la intensidad sobre el puerto 2 del MZ, obtenida por reflexión de las FBGs. (c) Señal fotodetectada en los puertos 3 y 4 del MZI para una condición de ajuste adecuada. (d) Intensidad de los puertos 3 y 4 para un desajuste del MZI.

FBGs presentan características espectrales similares (reflectividad, ancho de banda y longitud de onda de Bragg), como ya mencionamos anteriormente. En la Fig. 3.27 (b) se muestra la intensidad reflejada por el MZI en el puerto $P_{21}$, para una condición de ajuste. Las intensidades medidas en los puertos $P_{32}$ y $P_{42}$ pueden ser vistas en la Fig. 3.27 (c) y (d). La Fig. 3.27 (c) muestra un ajuste adecuado del MZI, alcanzando una diferencia 
de intensidades entre las ramas de aproximadamente $20 \mathrm{~dB}$ (o 100 veces). Esta condición se produce cuando las longitudes de camino óptico son ajustadas igualando las longitudes de las ramas con un error de $\pm 2 \mathrm{~mm}$ o menor. Sin embargo, como ya mencionamos, los cambios de temperatura producen un corrimiento en las características espectrales de las FBGs, modificando la respuesta de fase del dispositivo y generando un desajuste. Nuevamente, se debe emplear el concepto de repetitividad para obtener resultados homogéneos y comparables. En la Fig. 3.27(d) se observa la medida intensidad de los mismos puertos (3 y 4) pero bajo otra condición, para la cual se ha inducido un cambio de la polarización y/o birrefringencia sobre una de las ramas del filtro (enrollando la FO), alcanzando una diferencia de $10 \mathrm{~dB}$ entre las ramas (condición de desajuste de MZI).

Un desajuste o desbalance del MZI, que bajo estas condiciones es denominado también UMZI, puede ser utilizado para generar nuevas componentes espectrales en sistemas láser que trabajan en régimen APM, como describen en sus trabajos Vasseur y Li $[86,88]$. Bajo estos esquemas, el MZI funciona de dos maneras diferentes al mismo tiempo. Por un lado, permite modular las pérdidas dentro o fuera de una cavidad láser en configuración de anillo, mediante el empleo de un modulador de fase, induciendo una salida pulsada a intervalos de tiempo muy precisos. Por otra parte, el desbalance permite generar otras componentes de frecuencia, las cuales presentan un chirp debido a la dispersión de tercer orden introducida por una de las ramas de interferómetro, como se observa en la referencia [86]. De esta manera, el uso de las FBGs en la ecualización de la característica espectral de la emisión láser, en sistemas que operan en régimen APM y, en la generación de nuevas componentes espectrales, permite alterar el comportamiento temporal de estas señales.

Finalmente, la aplicación de estos dispositivos en sistemas de transmisión de canales WDM, requiere regularmente de todo tipo de compensaciones (de intensidad y fase) y sincronismo (para encaminar la información a diferentes puntos de una red óptica). En sistemas de comunicaciones, se emplean para "bajar" determinados canales en puntos intermedios de una red óptica y "subir" otros que desean ser retransmitidos (dispositivos add-drop) [89,90], evitando los problemas de la conversión OE y EO producidos por repetidores optoelectrónicos y sin necesidad de un corte del servicio [91,92]. 


\subsubsection{Implementación de líneas de retardo ópticas para un sis- tema de OBF}

En general, un haz electromagnético (EM) se puede formar en el espacio libre mediante la excitación de un elemento radiante o antena de radiofrecuencia $(\mathrm{RF})$, mientras que el direccionamiento del mismo se puede realizar desplazando la fase de las señales inyectadas a cada uno de los elementos de un conjunto de antenas, denominado phased-array antenna (PAA). Este conjunto de elementos radiantes genera una interferencia constructiva/destructiva en una cierta región del espacio (localizada sobre un plano, o un volumen, o bien empleando múltiples dimensiones), con el fin de dirigir la radiación en forma de haz en una dirección deseada, como describe en su libro Mailloux [93]. Un PAA se compone de un conjunto de antenas separadas regularmente a una distancia fija, en la que cada una de estas tiene asociado un desplazador de fase [94]. Los métodos para generar estos retardos de la fase son diversos y se denominan generalmente como sistemas de formación de haz o beamforming. En la Fig. 3.28 se puede observar un diagrama general, el cual contiene un PAA, módulos de potencia de RF conectados al PAA y un sistema de beamforming que permite producir cada uno de los retardos necesarios. Estos sistemas pueden ser desarrollados mediante técnicas analógicas de RF (basados en la electrónica de microondas), aunque nuevas técnicas emplean procesamiento digital y sistemas ópticos para generar los desplazamientos de fase o retardos, con el fin de mejorar la sensibilidad. Las aplicaciones relacionadas a los PAA son diversas y van desde los sistemas de radar [51,58], pasando por su aplicación en radio astronomía [57,95] y en sistemas distintos sistemas de comunicaciones [59,96].

En general, un obstáculo importante en el diseño de un sistema PAA con desplazadores de fase de banda ancha es que las características espectrales de los desplazadores analógicos presentan un ancho de banda limitado, como describe en su tesis Fakharzadeh [97]. Por lo tanto, cuando se producen desviaciones de la frecuencia de la portadora de microondas, el haz principal se desvía de la dirección deseada produciendo un estrabismo del mismo, también llamado squint [43]. Otra desventaja de los desplazadores de fase analógicos es que su pérdida de inserción no es balanceada (no es homogenea y depende de la frecuencia), lo 


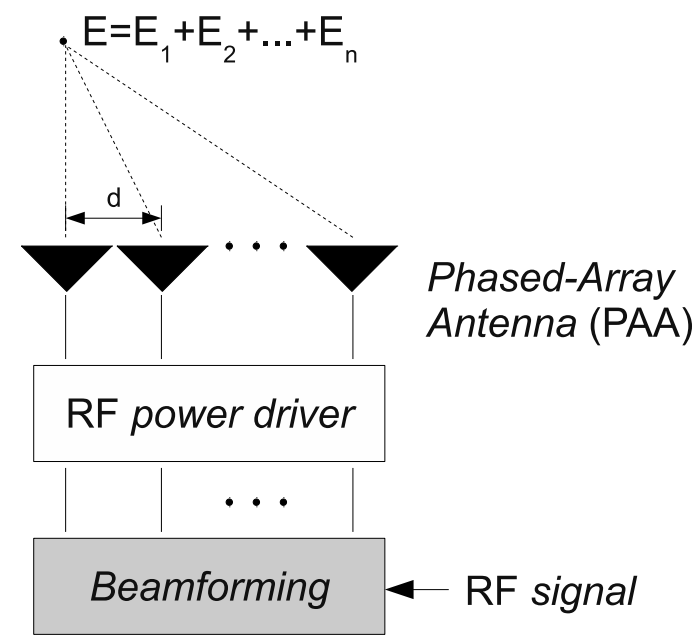

Figura 3.28: Esquema de un phased-array antenna (PAA) para la formación de haces de RF o beamforming. El procesamiento puede ser implementado mediante desplazadores de fase en el dominio analógico, de forma digital, o bien en el domino óptico mediante el diseño de líneas de retardo.

que implica que la respuesta de amplitud de la red de beamforming se ve alterada [98]. En general, estos sistemas presentan alguna de las siguientes características: las frecuencias de interés se extienden desde la banda L (1-2 GHz) hasta la banda X (alrededor de $8 \mathrm{GHz}$ ); la potencia de transmisión depende de la arquitectura y no existen limitaciones, van desde 10 W hasta 1 kW y más; el ancho de banda típico es comprendido entre 1-100 MHz (generalmente dependientes de la capacidad de muestreo de los conversores analógico/digitales AD empleados en la recepción); en transmisión se emplean señales pseudoaleatorias (PRBS) moduladas con señalización de fase binaria (BPSK); la potencia recibida en la antena es muy baja, del orden de $-100 \mathrm{dBm}$ y hasta el límite impuesto por la figura de ruido y la ganancia de procesamiento las cuales dependen de la modulación empleada, mientras la sensibilidad del receptor es determinada por el ancho de banda de predetección (ancho de banda del filtro de frecuencia intermedia). Actualmente se emplean tanto técnicas digitales como ópticas, por lo que el ancho de banda del filtro de una implementación digital (relacionado con el tiempo de integración) define también la sensibilidad de recepción. En los últimos años, la tecnología direct digital synthesis (DDS) de semiconductores ha 


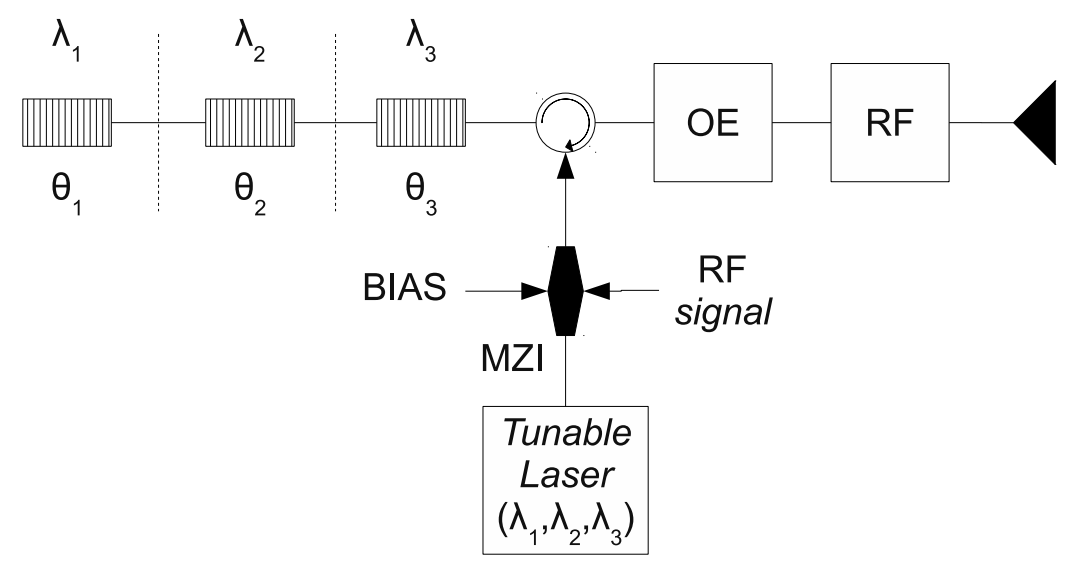

Figura 3.29: Línea de retardo ópticas basadas FO estándar y FBG. Se expresa una única línea o rama del sistema de OBF donde se muestran los principales componentes del mismo.

proporcionado una solución interesante en el procesamiento digital de las señales.

Una solución óptica en la implementación de las líneas de retardo puras brinda varias ventajas, algunas de las cuales son: incremento notable en la sensibilidad del sistema (procesamiento de señales de gran ancho de banda), inmunidad a fuentes de EMI externas y fácil implementación del sistema con elementos discretos de FO, entre otras. En general, un sistema de beamforming óptico (OBF) se basa en la utilización de líneas de retardo puras implementadas en el dominio óptico y denominadas como true-time delay (TTD), en lugar de los desplazadores de fase analógicos implementados mediante las técnicas anteriormente mencionadas [99]. De esta forma, para dirigir el haz EM por medio del PAA, es necesario sintonizar adecuadamente las distintas líneas de retardo que componen el OBF. Un conjunto de líneas de retardo de variación continua permite dirigir el haz EM en cualquier dirección (no sólo ángulos discretos), dentro de la región de cobertura acotada y de forma precisa.

Una implementación típica de las TTD se basa en el empleo de FBGs [100,101]. En la Fig. 3.29 se puede observar un esquema básico de esta configuración, donde se representa una única rama del sistema de OBF por simplicidad. El láser sintonizable en longitudes de onda es empleado para iluminar las FBGs de forma selectiva mientras que, a través de las TTDs, se generan los retardos de las señales ópticas que llegan luego la etapa de 
conversión OE. Un modulador del tipo MZI o bien de electroabsorción, se encarga de modular la portadora de luz, la cual se encuentra generalmente dentro de la banda C o L del espectro óptico, mediante una señal eléctrica externa de RF. El circulador encamina la luz de la fuente hacia las FBGs, las cuales producen una reflexión que depende de la longitud onda de la fuente láser. Luego, la señal óptica reflejada es retardada de forma diferente para cada una de las FBGs cuyas longitudes de onda de Bragg son iguales a $\lambda_{1}, \lambda_{2}, \ldots, \lambda_{n}$. El número de FBGs depende de la resolución del sistema OBF elegida (número de ángulos, en un rango limitado por el campo de visión (FOV) de alrededor de $120^{\circ}$ ). Por otra parte, el número de líneas de FO del OBF depende del PAA implementado. De esta manera, el conjunto de FOs y FBGs produce los retardos controlados para cada uno de los ángulos requeridos $\theta_{1}, \theta_{2}, \ldots, \theta_{n}$, y la señal reflejada por las FBGs es encaminada por los circuladores hacia la etapa de conversión OE (basada en fotodetectores y una etapa que consiste en un amplificador electrónico de transimpedancia). Luego, la señal eléctrica debe ser reacondicionada para ser inyectada adecuadamente en el PAA, donde se logra la conformación del haz electromagnético en una dirección establecida. El retardo de la onda electromagnética generada por dos elementos de antena vecinos (separados en una distancia $\left.d=\lambda_{R F} / 2=c /\left(2 f_{R F}\right)\right)$ se calcula mediante la siguiente ecuación

$$
\Delta t=\frac{d}{c} \sin (\theta)
$$

donde $d$ es la distancia entre elementos de la antena y $\theta$ es el ángulo producido entre los dos haces propagados. Luego, ese retardo temporal traducido al dominio óptico se expresa por medio de una distancia de propagación en la FO hasta alcanzar una de las FBGs, y se expresa como

$$
2 \Delta L=v_{\text {prop }_{F O}} \Delta t=\frac{c \Delta t}{n_{0}} \Longrightarrow \Delta L=\frac{\lambda_{R F} \sin (\theta)}{4 n_{0}}
$$

donde $v_{\text {prop }_{F O}}$ es la velocidad de propagación de una onda o campo electromagnético por una FO cuyo índice de refracción efectivo es $n_{0}$ y $\theta$ es igual al valor del ángulo de beamforming. 


\section{Simulación del sistema de OBF propuesto}

Con el fin de realizar una simulación del sistema OFB más cercana a la realidad, se utilizó una herramienta de cálculo de sistemas ópticos en el cual se tuvieron en cuenta la mayoría de los parámetros de los dispositivos discretos que serán mencionados a continuación.

En nuestro trabajo [43] se modeló un PAA de 4 antenas isotrópicas (4 FOs) y empleando 3 FBGs por cada FO, una para cada ángulo $\left(\theta_{1}=45^{\circ}, \theta_{2}=-45^{\circ}\right.$ y $\left.\theta_{3}=0^{\circ}\right)$. La distribución de las longitudes de onda de los láseres empleados se corresponden con las longitudes de onda de las FBGs utilizadas en el diseño de las TTD. Las FBGs empleadas presentan un valor de reflexión elevado, de $99 \%$ para las longitudes de onda de Bragg elegidas $\left(\lambda_{3}=1550\right.$ $\mathrm{nm}, \lambda_{2}=1551 \mathrm{~nm}$ y $\left.\lambda_{1}=1552 \mathrm{~nm}\right)$ y un ancho de banda medido a $3 \mathrm{~dB}$ igual a $0,2 \mathrm{~nm}$ (aproximadamente $25 \mathrm{GHz}$ ). Suponiendo que son FBGs uniformes, se las puede modelar con una longitud física del orden de $6 \mathrm{~mm}$ y una variación máxima de índice de refracción $\delta n=25 \times 10^{-5}$. En general, las FBGs introducen una pequeña distorsión de fase la cual será analizada al final de esta sección, relacionada a los resultados de [43]. La separación espectral entre las FBGs es igual a $1 \mathrm{~nm}$ para evitar problemas de crosstalk debido a la interferencia de los lóbulos laterales de las mismas, y además permite acotar los efectos del cambio de la velocidad de grupo (cuyo valor depende de la longitud de onda de la luz transmitida). Un espaciado menor se puede lograr empleando técnicas de apodización en el grabado de las FBGs. Sin embargo, un espaciamiento espectral mayor entre las FBGs provoca que algunos parámetros del sistema, como la velocidad de grupo $v_{g}$ y/o el $\beta_{2}$ de la FO, adquieran un valor diferente para cada longitud de onda, alterando la respuesta de fase del OBF. En esos casos, se debe recurrir a una ecualización de las TTD para minimizar los efectos de la distorsión introducida.

Suponiendo que la longitud de onda de la señal electromagnética propagada en el aire es $\lambda_{R F}=c / f_{R F} \approx 0,3 \mathrm{~m}$ (para $f_{R F}=1 \mathrm{GHz}$ ) y la distancia de separación entre elementos de la antena es $d=\lambda_{R F} / 2=0,15 \mathrm{~m}$, se puede estimar el diagrama de retardos. En la Tabla 3.1 se puede observar el diagrama de retardos temporales que produce un direccionamiento de 3 haces como los especificados, mientras que en la Tabla 3.2 se expresan las longitudes de 


\begin{tabular}{l|l|l|l|l|l}
$\theta\left[^{\circ}\right]$ & Retardo $[\mathrm{ps}]$ & $t_{1}[p s]$ & $t_{2}[p s]$ & $t_{3}[p s]$ & $t_{4}[p s]$ \\
\hline $0^{\circ}$ & 0 & 0 & 0 & 0 & 0 \\
$-45^{\circ}$ & $-353,55$ & $4 \Delta t=-1414,2$ & $3 \Delta t=-1060,65$ & $2 \Delta t=-707,1$ & $1 \Delta t=-353,55$ \\
$45^{\circ}$ & $|\Delta t|=353,55$ & $5 \Delta t=1767,75$ & $6 \Delta t=2121,3$ & $7 \Delta t=2474,85$ & $8 \Delta t=2828,4$
\end{tabular}

Tabla 3.1: Diagrama de retardos especificado por las FBGs para un sistema OBF de 3 ángulos y un PAA formado por 4 antenas isotrópicas. La primera fila indica los 4 retardos $\left(t_{n}\right.$ con $n=1,2,3$ y 4$)$ debidos a las FBGs ubicadas en una posición de referencia $(\Delta z=$ $0)$.

\begin{tabular}{l|l|l|l} 
Línea de OBF & $\theta_{3}=0^{\circ}$ & $\theta_{1}=-45^{\circ}$ & $\theta_{2}=45^{\circ}$ \\
\hline 1 & 0 & $4 \Delta z=145,19 \mathrm{~mm}$ & $5 \Delta z=181,49 \mathrm{~mm}$ \\
2 & 0 & $3 \Delta z=108,89 \mathrm{~mm}$ & $6 \Delta z=217,79 \mathrm{~mm}$ \\
3 & 0 & $2 \Delta z=72,59 \mathrm{~mm}$ & $7 \Delta z=254,09 \mathrm{~mm}$ \\
4 & 0 & $\Delta z=c \Delta t /\left(2 n_{0}\right)=36,29 \mathrm{~mm}$ & $8 \Delta z=290,38 \mathrm{~mm}$
\end{tabular}

Tabla 3.2: Ubicación espacial de las FBGs para un sistema OBF de 3 ángulos y un PAA formado por 4 antenas isotrópicas. La primera columna, ángulo $\theta_{3}=0^{\circ}$, expresa el punto de referencia desde donde se miden las distintas longitudes de FO entre las FBGs del OBF.

los tramos de FO entre las distintas FBGs.

En general, los dispositivos discretos empleados en el diseño del sistema de OBF fueron seleccionados del catálogo de Thorlabs. Se emplean 3 láseres de semiconductor modelo SFL1550P (longitudes de onda admisibles 1550-1562 nm, con un error absoluto de $\pm 0,5 \mathrm{~nm}$ ) de longitud de onda fija en vez de un láser sintonizable para simplificar el modelo numérico. El láser DFB presenta un valor aproximado de ancho de banda igual a $50 \mathrm{kHz}$, un valor de densidad espectral de ruido de intensidad relativa RIN igual a $-150 \mathrm{~dB} / \mathrm{Hz}$ y presenta una intensidad de emisión máxima de $50 \mathrm{~mW}$. El modulador seleccionado es un modulador Mach-Zehnder de $\mathrm{LiNbO}_{3}$ modelo LN56S y presenta un ancho de banda de $10 \mathrm{GHz}$. El fotodetector seleccionado modelo FGA04 presenta una responsividad relativamente plana 

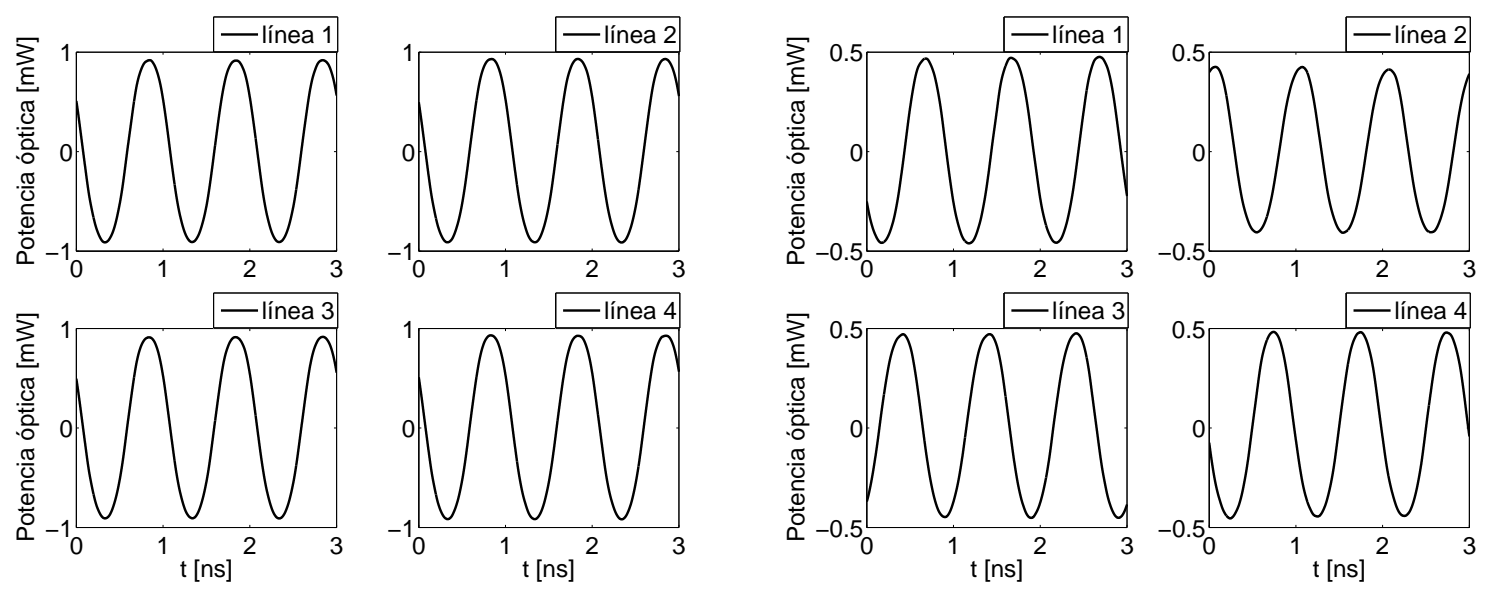

(a)

(b)

Figura 3.30: Señales fotodetectadas en la etapa de conversión OE, las cuales son eventualmente amplificadas para excitar el PAA. Se muestra la salida para (a) $\theta_{3}=0^{\circ}$ y (b) $\theta_{2}=45^{\circ}$.

en toda la banda de longitudes de onda utilizada, mientras que su valor es próximo a 0,9 $\pm 0,05 \mathrm{~A} / \mathrm{W}$ en $1550 \mathrm{~nm}$. Además, presenta un valor de dark current igual a $0,5 \mathrm{nA}$ para 5 $\mathrm{V}$ y una potencia equivalente de ruido (NEP) igual a $1,5 \times 10^{-15} \mathrm{~W} / \sqrt{\mathrm{Hz}}$ (potencia óptica mínima para generar una corriente eléctrica en los terminales del fotodetector, el valor se expresa en unidades de la PSD). El tiempo de subida y/o bajada es próximo a los 100 ps para $5 \mathrm{~V}$ y la capacidad de juntura es muy pequeña del orden de $1 \mathrm{pF}$ para $5 \mathrm{~V}$. El valor de la capacidad es un factor limitante en la detección de señales con gran ancho de banda. Un circulador estándar modelo 6015-3 presenta las siguientes características: rango de longitudes de onda de trabajo entre 1525-1610 nm, potencia máxima de 500mW, pérdida de inserción de $I L=0,8 \mathrm{~dB}$, pérdida de retorno $R L \geq 50 \mathrm{~dB}$ y una aislación mayor a $I=40 \mathrm{~dB}$. El acoplador seleccionado modelo FCQ1315 presenta una pérdida de inserción de $I L=0,2 \mathrm{~dB}$ y opera en un ancho de banda de $\pm 40 \mathrm{~nm}$ sobre una longitud de onda de trabajo de $1550 \mathrm{~nm}$. Particularmente, un acoplador $1 \times 4$ divide la potencia en partes iguales, por lo tanto se pierden alrededor de $6 \mathrm{~dB}$ adicionales (pérdida de inserción). Las señales fotodetectadas por la etapa de conversión OE pueden ser vistas en la Fig.3.30, donde se puede observar el retardo relativo de cada una de las señales eléctricas generadas. 


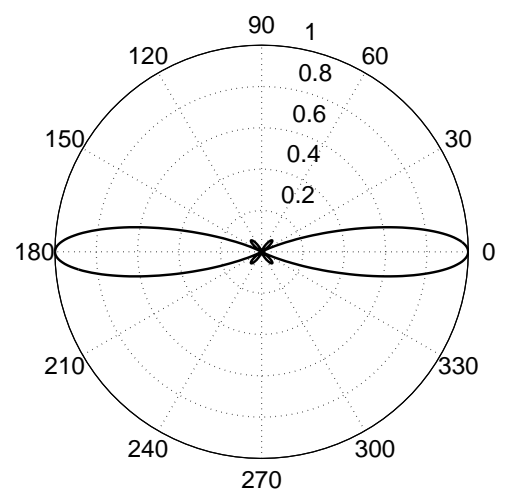

(a)

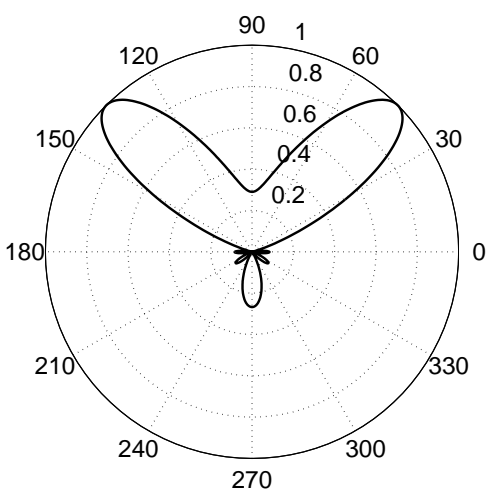

(b)

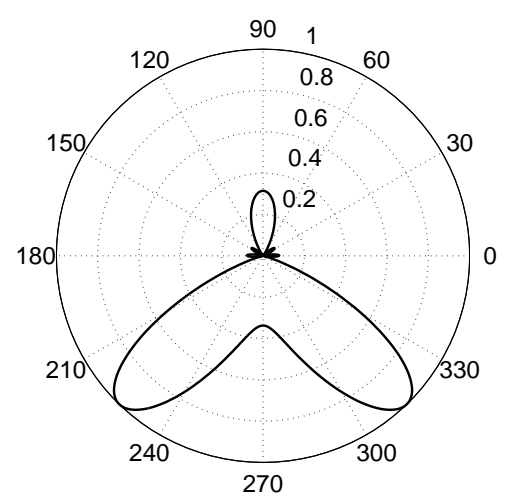

(c)

Figura 3.31: Generación de los haces electromagnéticos mediante un sistema OBF y un PAA formado por 4 antenas isotrópicas. Los diagramas muestran la potencia del campo resultante medida a $300 \mathrm{~m}$ del PAA, para los ángulos de diseño especificados. (a) $\theta_{3}=0^{\circ}$. (b) $\theta_{2}=45^{\circ}$. (b) $\theta_{1}=-45^{\circ}$.

Además, se observa que las señales presentan un muy bajo nivel de distorsión armónica para distintos valores de intensidad óptica evaluada, sobre un enlace de longitud igual a $500 \mathrm{~m}$ (entre el sistema OBF y el PAA). Las señales obtenidas mediante un fotodetector tipo PIN y un filtro de Bessel de cuarto orden, empleado para limitar el ruido presente en la señal fotodetectada [102], permiten generar los siguientes patrones de irradiación EM 
normalizados para un PAA medido a una distancia de $300 \mathrm{~m}$, como se puede observar en las Figs. 3.31. Como se mencionó de forma implícita en la Fig. 3.28, el campo eléctrico resultante se calcula como la suma de los campos eléctricos generados por cada uno de los elementos radiantes del PAA, mientras que la potencia de RF queda definida como $|E|^{2}=\left|E_{1}+E_{2}+\ldots+E_{n}\right|^{2}$, donde $E_{n}$ es el campo eléctrico transmitido por el $n$-ésimo elemento del PAA.

Una forma de estimar el desempeño del sistema propuesto es medir la relación portadora a ruido o CNR. Esta medida se logra obteniendo una relación entre la portadora y el piso de ruido, medido a $500 \mathrm{kHz}$ de la misma, según especifica la norma mencionada en el libro de Keiser [6]. Fijando la resolución espectral debida al procesamiento discreto, o sea $\Delta f=f_{s} / N$, donde $N$ es el número de muestras y $f_{s}$ la frecuencia de muestreo, se logra una resolución espectral igual a $40 \mathrm{kHz}$ y se logra medir un valor $\mathrm{CNR}=30 \mathrm{~dB}$, como se muestra en la Fig. 3.32 (a). La diferencia entre el valor establecido para la portadora y el medido está relacionado con los problemas de muestreo de las señales coherentes, para las cuales se debe emplear una gran cantidad de puntos $(N)$ y una frecuencia de muestreo elevada (lo que complica la medida pues las señales a muestrear son de alta frecuencia). A pesar de estos inconvenientes, como hemos visto, es posible establecer una cota aproximada del valor de CNR. Para evaluar el peso de la generación de armónicas en el sistema debidas a procesos lineales y no-lineales de sus componentes se estima la intermodulación de segundo orden y tercer orden (CSO y CTB, respectivamente) según se especifica en el libro de Pastor Abelián [103]. Para el cálculo de la CSO se fija la intensidad del láser a un valor minimo que permita medir dicho efecto (en nuestro caso $5 \mathrm{~mW}$ ) y se excita el modulador EO con dos portadoras, una de frecuencia $f_{1}=1 \mathrm{GHz}$ y otra igual a $f_{2}=1,1 \mathrm{GHz}$. El valor resultante de CSO para esta condición fue igual a 34,48 dB a (100 MHz y 2,1 GHz), como se muestra en la Fig. 3.32 (b). Estos valores son correspondientes a las frecuencias $f_{2}-f_{1}$ y $f_{1}+f_{2}$. Finalmente, la intermodulación de tercer orden (correspondientes a las frecuencias $2 f_{1}-f_{2}=900 \mathrm{MHz}$ y $2 f_{2}-f_{1}=1,2 \mathrm{GHz}$ ) resulta en un valor igual a $29,3 \mathrm{~dB}$. De esta manera, los valores medidos para la CNR, CSO y CTB son adecuados a lo que indican las normas mencionadas en $[6,103] \mathrm{y}$, para este caso, el efecto de las no-linealidades del 


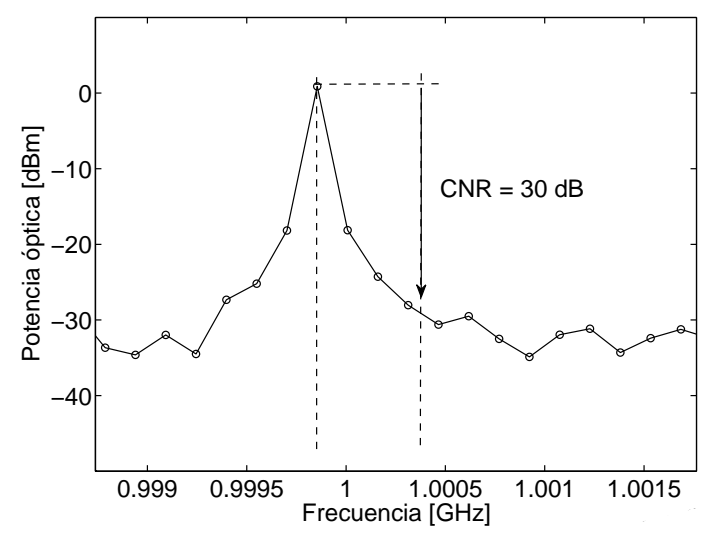

(a)

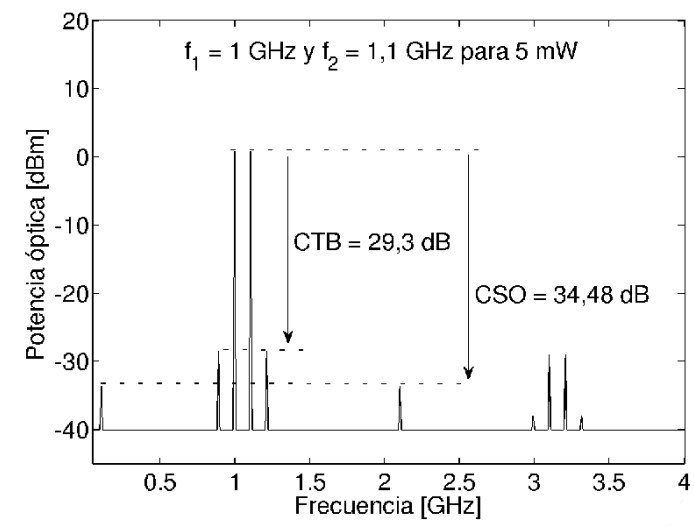

(b)

Figura 3.32: (a) Estimación de la relación portadora ruido del sistema OBF. (b) Medida de distorsión de segundo orden y de tercer orden relacionada a los productos de intermodulación mencionados.

sistema puede ser despreciado.

Finalmente, en nuestro trabajo [43], se introduce una discusión respecto del efecto de las características de fase de las FBGs y por consecuente en la generación del haz EM producido por el PAA. Se estudiaron tres posibles variables como: el cambio de la longitud de onda de Bragg de los filtros empleados, la desviación de la longitud de los tramos de FO que definen los retardos para cada caso y la modificación de la longitud de onda de los láseres. Todos los parámetros mencionados fueron tratados de forma estadística mediante el uso de distintas distribuciones normales, con valores típicos para la media y la desviación estándar. A partir de los resultados obtenidos mediante simulaciones, se puede observar que un error en la longitud de onda de Bragg con desviación estándar de 0,066 nm (aproximadamente un tercio del ancho de banda) produce la mayor distorsión en el patrón de irradiación, mientras que los desajustes de otros parámetros no tienen mucha influencia para los valores típicos considerados. Teniendo en cuenta este resultado, se debe prestar especial atención en la fabricación de las FBGs, con el fin de obtener un patrón de irradiación lo más cercano posible del ideal. 


\subsubsection{Método de caracterización de pulsos basado en un dispo- sitivo fotónico y dispersivo}

En esta sección se propone y analiza un nuevo método para caracterizar pulsos de luz tanto en intensidad como en fase, basado en una de las propiedades de función de distribución de Wigner (WDF). Esta función permite representar señales mediante un espacio de fase tiempo-frecuencia, como se describe en nuestro trabajo [44] y previamente en [104]. Para la implementación de este sistema se utilizan dispositivos ópticos pasivos tales como: acopladores y circuladores de FO, detectores de semiconductor y una FBG no-uniforme (LCFBG), que introduce una cierta cantidad de dispersión a los pulsos de entrada de forma controlada. La recuperación de la información completa del pulso (amplitud y fase) permite llevar a cabo dos aplicaciones muy importantes en el área de las telecomunicaciones. Por un lado, si el pulso recuperado se trata de un pulso transmitido por medio de una FO, se pueden realizar estimaciones de los parámetros responsables de la distorsión del mismo, lo que brinda información de la respuesta general del canal de transmisión. En este sentido, la información obtenida del mismo podría servir en el diseño de nuevos dispositivos que permitan llevar a cabo operaciones de reformateo, ecualización y/o compresión de los pulsos detectados.

En general, la elección del elemento dispersivo es la parte crítica del método propuesto. Una LCFBG es un dispositivo compacto que permite introducir una dispersión controlada mediante sus parámetros de diseño con una muy baja atenuación. Los parámetros constructivos de la LCFBG, relevantes para la implementación del método son: la longitud, la máxima variación del índice de refracción y el período de grabado que determinan las características de la LCFBG. Mediante estos parámetros se puede estimar el ancho de banda y la pendiente del retardo de grupo de dicho dispositivo, los cuales se encuentran unívocamente relacionados. La elección de la pendiente del retardo de grupo de la LCFBG permite determinar el factor de modulación del espectro $\Phi_{20}^{r}$ de la señal que se desea analizar. La desventaja de emplear este tipo de dispositivos puede ser observada en la distorsión que introduce al sistema de recuperación de los pulsos mediante un ripple que 
se encuentra presente en la característica del retardo de grupo de la LCFBG, inherente a estos componentes. Sin embargo, como podremos observar, un proceso de apodización de la LCFBG, realizado durante la fabricación de la misma, permite una recuperación de los pulsos adecuada con un error muy pequeño.

\section{Descripción del método}

El método está basado en la relación que existe entre el momento de primer orden de la WDF asociada a una señal y su frecuencia instantánea, expresión que se escribe por comodidad en función de la frecuencia angular $\omega=2 \pi f$, como Ec. 3.35.

$$
\int_{-\infty}^{\infty} \omega W_{u}(t, \omega) d \omega=\frac{\partial \phi(t)}{\partial t}|u(t)|^{2}
$$

siendo $W_{u}(t, \omega)$ la WDF asociada a la señal $u(t)=|u(t)| \exp (i u(t))$, definida como

$$
\begin{gathered}
W_{u}(t, \omega)=\int_{-\infty}^{\infty} u(t+\tau / 2) u^{*}(t-\tau / 2) e^{-i \omega \tau} d \tau \\
=\frac{1}{2 \pi} \int_{-\infty}^{\infty} U(\omega+\Omega / 2) U^{*}(\omega-\Omega / 2) e^{-i \Omega t} d \Omega,
\end{gathered}
$$

donde $U(\omega)$ representa la transformada de Fourier de $u(t)$.

El dominio de la WDF asociada a una señal cuya fase espectral es modulada en forma cuadrática, o sea $H(\omega)=\exp \left(-\Phi_{20}^{r} \omega^{2} / 2\right)$ y sufre un desplazamiento en el sentido del eje temporal, es decir,

$$
W_{u_{f}}(t, \omega)=W_{u}\left(t-\Phi_{20}^{r} \omega, \omega\right),
$$

siendo $W_{u}$ y $W_{u_{f}}$ las WDFs asociadas a las señales original y filtrada, respectivamente. La intensidad temporal o instantánea de la señal modulada se puede escribir como

$$
I_{u_{f}}(t)=\left|u_{f}(t)\right|^{2}=\int_{-\infty}^{\infty} W_{u_{f}}(t, \omega) d \omega=\int_{-\infty}^{\infty} W_{u}\left(t-\Phi_{20}^{r} \omega, \omega\right) d \omega,
$$

Derivando la Ec. 3.37 respecto del coeficiente de modulación $\Phi_{20}^{r}$ se puede demostrar que

$$
\frac{\partial I_{u_{f}}(t)}{\partial \Phi_{20}^{r}}=-\frac{\partial}{\partial t}\left[\int_{-\infty}^{\infty} \omega W_{u} d \omega\right]
$$


Finalmente, si se reemplaza la Ec. 3.35 en esta última, se obtiene

$$
\frac{\partial I_{u_{f}}(t)}{\partial \Phi_{20}^{r}}=-\frac{\partial}{\partial t}\left[I_{u}(t) \frac{\partial \phi(t)}{\partial t}\right]
$$

La Ec. 3.40, que es a veces llamada ecuación de transporte de intensidad temporal, ha sido utilizada para medir cambios en la fase temporal inducidos por la SPM y XPM, como muestra en su trabajo Dorrer et.al. [105]. Empleando la Ec. 3.40 es posible recuperar la fase de una señal, sin embargo, para obtener un procedimiento realizable es necesario introducir ciertas aproximaciones. Primero, la derivada respecto del coeficiente de modulación puede ser reemplazada por una aproximación en diferencias finitas centrada como

$$
\frac{\partial I_{u_{f}}(t)}{\partial \Phi_{20}^{r}} \cong \frac{\left.I_{u_{f}}(t)\right|_{\Phi_{20}^{r}}-\left.I_{u_{f}}(t)\right|_{-\Phi_{20}^{r}}}{2 \Phi_{20}^{r}}
$$

La modulación de fase espectral cuadrática puede ser producida mediante la transmisión de la señal a través de una FO compensadora de la dispersión o por reflexión en una LCFBG, siendo $\Phi_{20}^{r}$ el coeficiente de dispersión de segundo orden por unidad de longitud $\left(\beta_{2}\left(\omega_{0} L\right)\right)$ de alguno de los dispositivos para la frecuencia angular $\omega_{0}$. De esta manera, la derivada de la intensidad temporal puede ser implementada realizando dos detecciones temporales de la señal, cada una afectada por la misma magnitud de dispersión de segundo orden, pero de signos opuestos. Aprovechando esta implementación, la intensidad $I_{u}(t)$ puede ser aproximada por el promedio de las dos detecciones de la señal dispersada como se muestra en la Ec. 3.42.

$$
I_{u}(t) \cong \frac{\left.I_{u_{f}}(t)\right|_{\Phi_{20}^{r}}+\left.I_{u_{f}}(t)\right|_{-\Phi_{20}^{r}}}{2}
$$

Las Ecs. 3.41 y 3.42 sólo son válidas cuando el coeficiente de dispersión de segundo orden $\Phi_{20}^{r}$ posee un valor pequeño. Para encontrar restricciones al valor de $\Phi_{20}^{r}$ se considera un pulso con anchos temporal y espectral $\Delta t$ y $\Delta \omega$, respectivamente. Si se tiene en cuenta que $\Phi_{20}^{r}$ es igual a la tangente del ángulo de desplazamiento o "cizalladura", se puede demostrar fácilmente que el coeficiente de dispersión de segundo orden debe ser mucho menor que la razón del ancho temporal respecto del ancho espectral, es decir, 


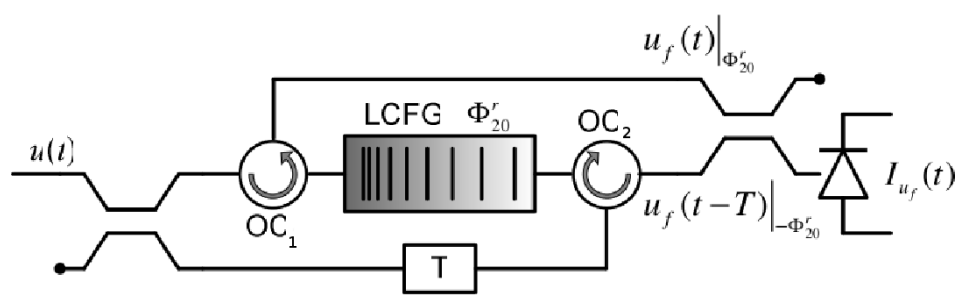

Figura 3.33: Diagrama esquemático de la implementación del dispositivo que permite recuperar la fase y la intensidad de un pulso a partir de dos detecciones de la intensidad del pulso dispersado. La LCFBG presenta un parámetro de dispersión de segundo orden $\Phi_{20}^{r}$. $\mathrm{OC}_{1}$ y $\mathrm{OC}_{2}$ : circuladores ópticos.

$$
\Phi_{20}^{r} \ll \frac{\Delta t}{\Delta \omega} .
$$

La Fig. 3.33 muestra un diagrama esquemático del sistema propuesto para recuperar la fase y la amplitud de una señal. El pulso de entrada $u(t)$ es dividido en dos señales por el acoplador de relación 50:50. Un pulso, luego de propagarse por el circulador óptico de la izquierda $\left(\mathrm{OC}_{1}\right)$, se refleja en el extremo izquierdo de la LCFBG y es encaminado nuevamente por el $\mathrm{OC}_{1}$ para ser detectado luego de pasar por el acoplador de relación 50:50 de la derecha. Un segundo pulso, que es una versión retardada del pulso de entrada, es retardado un tiempo $T$ mayor a la duración temporal del pulso de entrada, mientras que de haber circulado por $\mathrm{OC}_{2}$, experimentaría una dispersión de segundo orden de signo opuesto cuando se reflejaría en el extremo derecho de la LCFBG. Así, luego de dos detecciones consecutivas (retardadas un tiempo igual a $T$ segundos) de la señal dispersada, la fase y la intensidad de $u(t)$ pueden calcularse empleando las Ecs. 3.40, 3.41 y 3.42.

\section{Resultados numéricos}

Para evaluar la influencia del ripple de una LCFBG en el desempeño del método implementado por el sistema de la Fig. 3.33, se considera la recuperación de un pulso de tipo secante hiperbólica con duración media cuadrática (RMS) $T_{0}=100$ ps que presenta variación lineal de su frecuencia (chirp lineal con parámetro $C=6$ ), similar al resultado numérico obteni- 


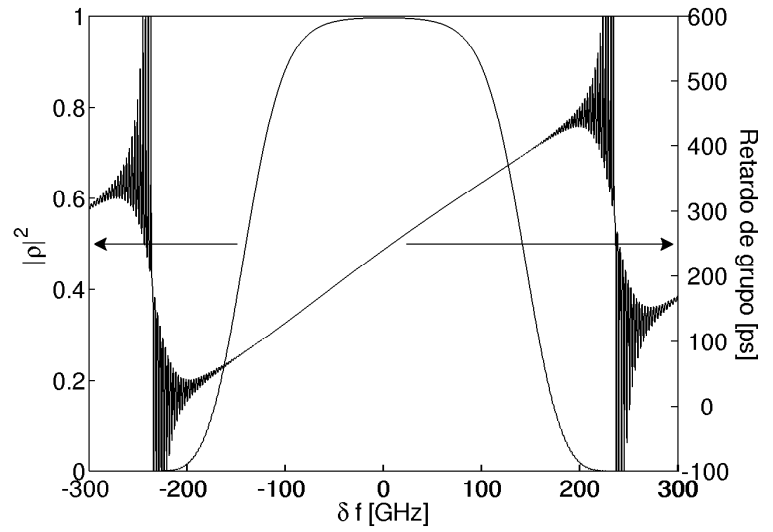

(a)

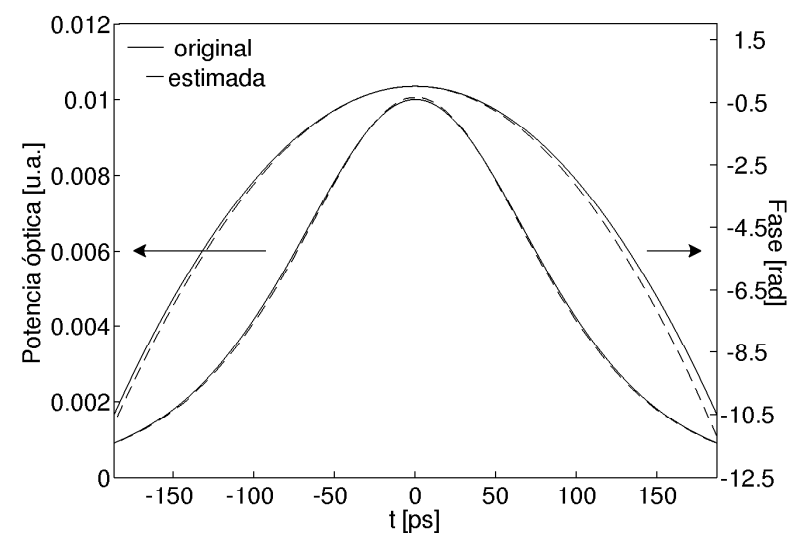

(c)

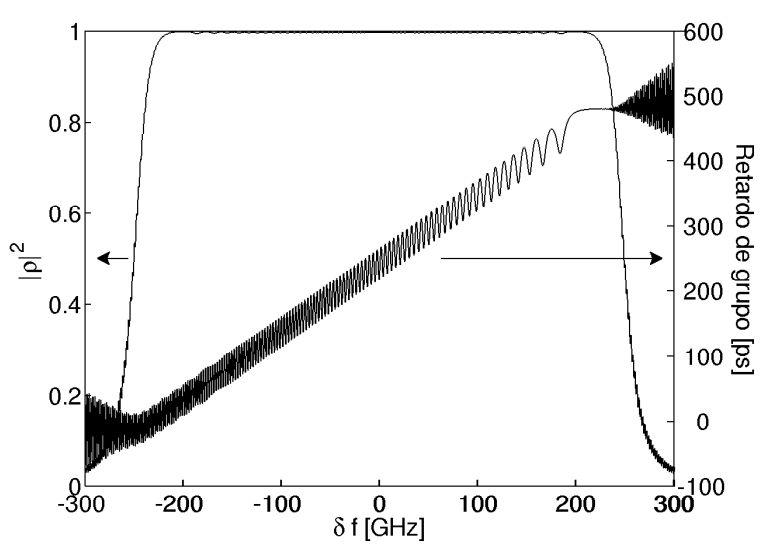

(b)

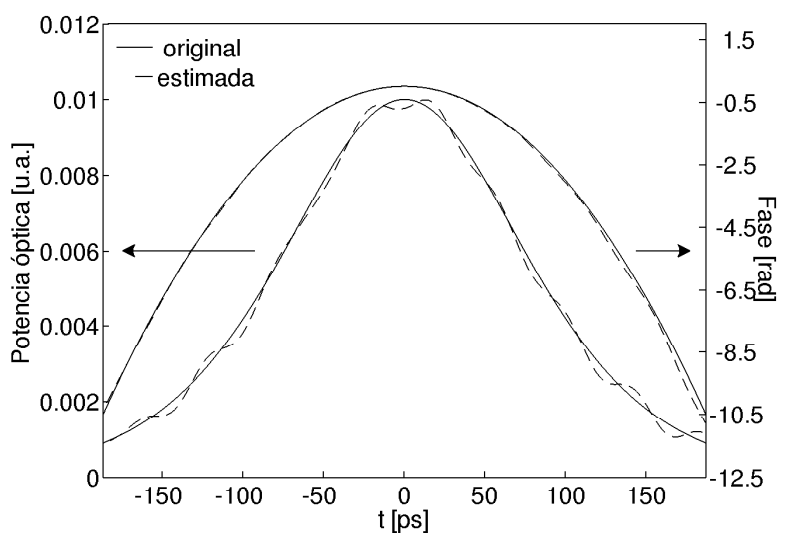

(d)

Figura 3.34: Resultados de la recuperación de un pulso de tipo secante hiperbólica de duración $T_{0}=100$ ps y chirp lineal con parámetro $C=6$. (a) y (b) muestran la reflectividad y el retardo de grupo con pendiente $\Phi_{20}^{r}=0,166 \mathrm{ps} / \mathrm{GHz}$ de una LCFBG de $5 \mathrm{~cm}$ de longitud con apodización de tipo coseno elevado y sin apodización, respectivamente. (c) y (d) muestran la intensidad y la fase originales (en línea llena) y recuperadas (en línea a trazos), para los casos especificados en (a) y (b), respectivamente.

do a la salida de un láser de cavidades acopladas que opera bajo un régimen de operación tipo APM. La detección de las intensidades se realiza mediante un promedio de 100 intensidades para una relación señal a ruido $\mathrm{SNR}=25$ dB. En las Figs. 3.34 (a) y (b) se muestran la reflectividad y el retardo de grupo de una LCFBG de $5 \mathrm{~cm}$ de longitud con 
apodización de tipo coseno elevado y sin apodización, respectivamente, ambas con pendiente del retardo de grupo $\Phi_{20}^{r}=0,166$ ps/GHz. En las Figs. 3.34 (c) y (d) se muestran la potencia óptica y la fase originales (en línea llena) y recuperadas (en línea a trazos), cuando la LCFBG del sistema es la correspondiente a las mencionadas en las Figs. 3.34 (a) y (b), respectivamente. Se puede observar que la fase del pulso es recuperada satisfactoriamente con ambos dispositivos. Esto se debe al efecto de promediado que introducen las integraciones necesarias para el cálculo de la fase. Sin embargo, la influencia del ripple de la LCFBG se vuelve apreciable en la recuperación de la potencia óptica del pulso cuando ésta es aproximada por la Ec. 3.42, como se puede observar a partir de la comparación de las Figs. 3.34 (c) y (d). En el caso de emplear el método con una red cuyo ripple no puede ser despreciado, es posible emplear un acoplador extra y otro detector de gran ancho de banda para obtener la intensidad a través de una medida directa.

\section{Discusión de los resultados numéricos}

El sistema de caracterización propuesto está basado en una propiedad de la WDF. En principio es aplicable a cualquier tipo de pulso o señal. Sin embargo, las aproximaciones que fueron hechas para poder lograr una implementación fotónica del sistema y las restricciones introducidas por los componentes involucrados, limitan su rango de operación. En principio, la primera restricción que existe es debida al ancho de banda del detector utilizado que delimita la duración mínima de los pulsos que pueden ser caracterizados. Por ejemplo, si se emplea un detector con un ancho de banda de $10 \mathrm{GHz}$, la duración mínima de los pulsos estará acotada a 100 ps, aproximadamente. La segunda restricción, y quizás la más obvia, es el ruido tanto de detección como el propio de la señal óptica. Este inconveniente puede salvarse en parte a partir del procesamiento de señales, en una primera aproximación, como se mostró en los resultados numéricos, promediando la detección de múltiples pulsos. Sin embargo, esta forma de procesamiento es válida bajo la hipótesis de que las características de la intensidad y fase de los pulsos sean aproximadamente invariantes en el tiempo. De todos modos, aún cuando se promedien las detecciones para reducir el 
efecto del ruido, el sistema presenta una limitación cuando se pretende caracterizar pulsos con duraciones grandes, aún cuando se cumple la condición establecida por la Ec. 3.43, es decir el cociente $\Delta t / \Delta \omega$ es mucho mayor que el coeficiente de dispersión del sistema $\Phi_{20}^{r}$. En estos casos, la aproximación a la derivada de la Ec. 3.41 se vuelve inestable debido a un efecto de amplificación de los errores introducidos por el ruido de manera que el error efectivo cometido al obtener la forma de onda de fase crece, limitando el rango de operación para pulsos de duraciones largas, con relaciones señal a ruido no tan favorables $(S N R<25$ dB). Una solución a este inconveniente parte de suponer un modelo de ruido apropiado y estudiar sus efectos en el sistema de caracterización, aunque estos tópicos serán analizados en un trabajo futuro.

La implementación propuesta para el sistema requiere el empleo, principalmente, de la aproximación a la derivada de la intensidad respecto del coeficiente de dispersión de la Ec. 3.41 y de la aproximación a la intensidad de la Ec. 3.42, aunque esta última puede ser obviada si se emplea un acoplador extra y otro detector (o dos acopladores y el mismo detector) para medir la intensidad directamente. Para que dichas ecuaciones sean válidas, se debe cumplir la condición indicada en la Ec. 3.43. Esto impone otro límite a la duración mínima de los pulsos. Si se considera la caracterización de un pulso gaussiano con $\Delta t_{F W H M}=1,67 T_{0}$ (siendo $T_{0}$ el ancho temporal RMS), su ancho espectral será $\Delta \omega_{F W H M}=1,67 T_{0}^{-1}$, con lo que $\Delta t / \Delta \omega=T_{0}^{2}$ si la fase del pulso es constante (no tiene chirp). Entonces, por ejemplo, para un dado sistema con coeficiente $\Phi_{20}^{r}=0,166 \mathrm{ps} / \mathrm{GHz}$ como el empleado en la sección anterior, se podrían caracterizar pulsos gaussianos limitados por transformada con duraciones mayores a $\Delta t_{F W H M}=68 \mathrm{ps}\left(T_{0}=41 \mathrm{ps}\right)$, aunque en ese caso el sistema no sería de gran utilidad. En consecuencia, para el sistema ejemplo planteado, y dependiendo de las variaciones de fase que presente el pulso, la duración mínima de los pulsos debería tener un valor estimado mayor a 100 ps.

En general, todos los elementos utilizados para implementar el sistema introducen alguna limitación. Los elementos principales que reducen considerablemente el rango de operación del sistema son el detector y la LCFBG. Ya fue mencionado en la sección anterior, como una LCFBG que presenta un ripple apreciable, distorsiona las formas de onda recuperadas, 
principalmente la de intensidad. También es necesario considerar que, cuando se quieren caracterizar pulsos de duraciones pequeñas, su correspondiente cociente $\Delta t / \Delta \omega$ será pequeño y por consiguiente, la pendiente del retardo de grupo de la red deberá tener un valor menor aún. Este hecho trae aparejado un inconveniente constructivo de la LCFBG puesto que, para tener pendientes del retardo de grupo de reducido valor, se requieren anchos de banda muy grandes. Es posible estimar que, para poder recuperar pulsos con duraciones del orden de las decenas de picosegundos, serían necesarias FBGs cuyas longitudes podrían ser menores a un centímetro. En una primera aproximación, esta limitación puede ser mitigada en una implementación del sistema como la propuesta en [106], donde se emplea FO monomodo de dispersión desplazada (NZDS-SMF) y FO compensadora de la dispersión (DCF) como elemento dispersivo, obteniendo valores pequeños de $\Phi_{20}^{r}$. 


\section{Capítulo 4}

\section{Modelado de un láser APM pasivo de cavidades acopladas}

Los láseres son dispositivos que generan o amplifican una radiación de forma coherente, para las regiones del espectro electromagnético cuyas frecuencias son del infrarrojo, visible, ultravioleta y/o rayos X. Estos operan mediante un principio general que fue originalmente inventado para frecuencias en el rango de las microondas, también llamado microwave amplification by stimulated emission of radiation (MASER) [107-109]. Naturalmente, desde el surgimiento de las frecuencias ópticas, se habla de amplificación de la luz mediante la emisión estimulada de la radiación (LASER) [110-112]. Este principio es aplicado a una enorme variedad de dispositivos y sistemas (basados en distinto tipo de materiales, métodos de excitación y enfoques de diseño) que operan en diversas regiones del espectro electromagnético. Los láseres de FO en particular, presentan muchas ventajas frente a sus antecesores, implementados mediante dispositivos ópticos tipo bulk-optics. Actualmente, son generalmente aplicados en implementaciones robustas y compactas orientadas a las comunicaciones, el sensado de múltiples variables del mundo físico (presión, temperatura, deformación y propiedades ópticas, entre otras), el maquinado de materiales y en diversas industrias, como la automotriz, defensa e inclusive la aeroespacial.

En este capítulo, se introduce en la Sección 4.1 el diseño de una cavidad láser, el cual 
emplea FBGs como dispositivos de realimentación del proceso de generación de la emisión estimulada láser mediante el empleo de un medio activo basado en una FO dopada con $\mathrm{Er}^{3+}$. Luego se introducen algunos regímenes de funcionamiento de los láseres y se establecen los principios del régimen de operación mode locking aplicado a un sistema láser de FO formado por dos cavidades acopladas que emplea FBGs. En la Sección 4.2 se presenta un modelo analítico, base de este trabajo de tesis que, mediante algunos supuestos, permite describir la salida pulsada del láser, obteniendo una solución aproximada mediante el uso de una herramienta de cálculo simbólico. En la Sección 4.3 el modelo analítico es resuelto de forma numérica mediante un algoritmo que emplea el SSM de forma conjunta con una matriz de scattering, con el fin de calcular la propagación de los campos en ambas cavidades del láser, como se muestra en un trabajo que hemos publicado recientemente [2]. El algoritmo implementado tiene en cuenta las características espectrales de las FBGs (en modulo y fase) de tal forma que permite resolver el acoplamiento de los campos que concurren a una única FBG común a ambas cavidades. Mediante este modelo se analizan las propiedades lineales y no-lineales del sistema y se establecen las condiciones de estabilidad del mismo. La comparación entre estos resultados numéricos y los obtenidos de forma experimental es desarrollada en el Capítulo 5. Finalmente, en la Sección 4.4 se discuten los resultados numéricos obtenidos mediante el modelo propuesto en la Sección 4.3.

\subsection{Introducción}

En general, los elementos que constituyen un sistema láser son: i) un medio de ganancia (medio activo); ii) un proceso de bombeo o excitación de dicho medio; y iii) elementos de realimentación óptica adecuados que componen la cavidad láser, donde la emisión producida se reinyecta y filtra en forma de modos de (frecuencias discretas) que se estabilizan en dicho sistema. En la Fig. 4.1 se muestra el esquema general de un láser, donde un proceso de bombeo del medio activo es requerido para excitar los átomos, iones o moléculas que componen el material de un nivel de energía inferior a otro más elevado. El proceso de emisión estimulada ocurre cuando se incrementan los niveles de población de iones dichas 


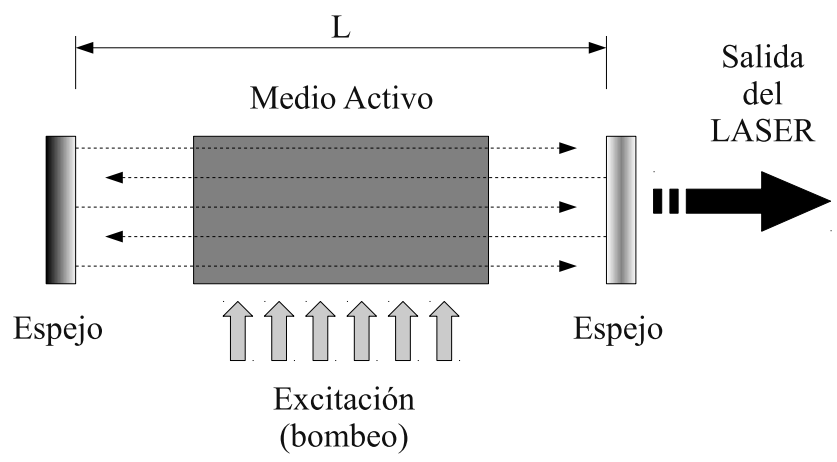

Figura 4.1: Esquema general de una cavidad LASER.

especies en el estado excitado y se produce un proceso de inversión de la población, para el cual parte de la energía decae de forma radiativa emitiéndose en forma de luz coherente. En la práctica para que esto suceda, se debe cumplir una condición en la que la ganancia del medio (dependiente de las poblaciones involucradas en el proceso láser y de las probabilidades de transición respectivas [14]) supera a las pérdidas en la cavidad. Esta condición se puede obtener mediante diversas configuraciones y con una amplia variedad de materiales. Una vez que se alcanza la condición umbral (para la cual las pérdidas igualan a la ganancia), la radiación electromagnética generada dentro de un cierto rango angosto de frecuencias es amplificada de forma coherente al propagarse a través del medio activo. En general, la ASE se extiende en un rango de frecuencias generado por la transición de un nivel de energía superior hasta otro nivel de energía inferior, determinado principalmente por las probabilidades de ocurrencia de dicho proceso y de la población de los niveles de energía. Los elementos de realimentación son espejos, o dispositivos similares, que reflejan parte de la emisión y que están ubicados a ambos lados del medio de ganancia. Debido a las características de los procesos que lo generan, el haz de salida resulta aproximadamente monocromático y con una dirección muy bien definida. El estudio de los sistemas láser es bien conocido y ha sido un tópico de interés para muchos investigadores, y particularmente para Siegman, como describe minuciosamente en su libro [113]. 


\subsubsection{Diseño de las cavidades}

Las cavidades de los láseres de FO pueden ser diseñadas de diversas formas, como describen Digonnet y Hodgson en sus libros [15,114]. El tipo más común de cavidad es del tipo FabryPerot, que se realiza ubicando el medio de ganancia entre dos espejos de alta reflectividad, de una forma análoga a la descrita en el Capítulo 3. La frecuencia fundamental de un láser, el cual presenta una configuración similar a la observada en la Fig. 4.1, queda definida mediante el cálculo de la frecuencia de resonancia como

$$
f_{\text {laser }}=\frac{1}{t_{\text {roundtrips }}}=\frac{c}{2 n_{\text {eff }} L},
$$

donde $t_{\text {roundtrips }}$ es el tiempo que tarda una onda en recorrer la cavidad de longitud $L$, partiendo de un extremo, llegando al otro y retornando al mismo punto de partida (viaje de ida y vuelta o roundtrips). En general, los dispositivos empleados como espejos pueden presentar valores bajos o altos de reflectividad (aproximadamente entre un 30-95\%) para las longitudes de onda de emisión del láser, siendo al mismo tiempo transparentes a la radiación de la excitación aplicada. Una vez obtenida la emisión estimulada en un láser de FO, por medio de la transmisión de uno de los dispositivos que forman el sistema de realimentación, es necesario desacoplar la radiación de la excitación o bombeo (la cual es indeseable en la mayoría de los casos). La forma estándar de desacoplar esta radiación es utilizando un acoplador de FO del tipo WDM. Un parámetro importante en el diseño de la cavidad es el tiempo de vida promedio de un fotón (que viaja de un lado al otro de la misma), el cual está definido por

$$
\bar{t}_{\text {photon }}=-\frac{t_{\text {roundtrips }}}{\log _{10}\left(R_{1} R_{2}\left(1-l_{g}\right)\right)},
$$

donde $R_{1}$ y $R_{2}$ son los valores de reflectividad de los dispositivos que conforman el sistema de realimentación (evaluados en la longitud de onda de la emisión $\lambda_{\text {laser }}=c / f_{\text {laser }}$ ) y $l_{g}$ son las pérdidas del medio de activo. De esta manera se puede imponer un límite de coherencia temporal de la señal para valores de tiempo mayores al de vida media del fotón dentro de la cavidad del láser. Por ejemplo, para el sistema láser que será discutido 
en este capítulo, una longitud de cavidad típica del orden de $L=2 \mathrm{~m}$, con un valor de reflectividad promedio igual a $93 \%$, resulta en $t_{\text {roundtrips }} \approx 19$ ns y $\bar{t}_{\text {photon }} \approx 16 t_{\text {roundtrips }}=$ 300 ns para un nivel de pérdidas despreciable, mientras que para niveles de pérdida mayores $\bar{t}_{\text {photon }}<10 t_{\text {roundtrips. }}$ El límite de coherencia temporal establecido de esta manera nos brinda información respecto al tiempo mínimo que podremos representar de forma adecuada por medio de nuestro modelo analítico, cambios en la intensidad y forma de los pulsos, sin cometer errores sistemáticos adicionales.

Una forma de generar una realimentación adecuada de la emisión láser es mediante el uso de FBGs como espejos, como se discutió en el Capítulo 3, lo que permite mejorar la eficiencia de un láser de $\mathrm{FO}$ dopada con $\mathrm{Er}^{3+}$ (EDFL) frente a otros sistemas láser basados en dispositivos de óptica espacial o bulk-optic, como muestra en su trabajo Kashyap et.al. [115]. La configuración más sencilla emplea dos FBGs en cada uno de los extremos de una FO dopada con $\mathrm{Er}^{3+}$, formando una cavidad Fabry-Perot activa [116,117]. Otros dispositivos, como los DFB basados en FBGs, son utilizados en láseres para sintonizar de forma continua la longitud de onda de la emisión con un ancho de línea muy estrecho [118]. Además, pueden oscilar en un único modo longitudinal o en múltiples frecuencias, mediante el ajuste adecuado de la longitud de la FO empleada entre ambas FBGs. De una forma similar, aunque prescindiendo de un medio activo, hemos diseñado dispositivos fotónicos aptos para tal fin [39]. El uso de tres FBGs conectadas en serie permite generar un sistema láser de dos cavidades tipo Fabry-Perot acopladas, donde el sistema admite alteraciones pequeñas de la longitud de las cavidades mediante un autoajuste de la longitud de onda, el cual está relacionado a la característica de la dispersión de las FBGs (longitud de penetración efectiva), como muestra en su trabajo Huang et.al. [22]. Otras configuraciones emplean las características espectrales de un interferómetro de Mach-Zehnder como elemento para controlar las pérdidas de forma selectiva dentro de una cavidad de anillo, con el fin de sintonizar la longitud de onda de la emisión en un rango mayor a $39 \mathrm{~nm}$ [119]. El nivel de las pérdidas de la cavidad se puede modificar mediante el control de la diferencia de camino óptico introducida por el cambio de la longitud de una de las ramas del interferómetro, de una forma similar a la vista en el Capítulo 3. Estos filtros son empleados también 
para modular las pérdidas de forma sincronizada con la emisión estimulada alcanzando tiempos de jitter menores a 130 fs en trenes de pulsos de hasta $40 \mathrm{GHz}$ de frecuencia de repetición y anchos de pulso de 1 ps, en régimen mode locking activo y de longitud de onda sintonizable en un rango de $30 \mathrm{~nm}$ [41]. En general, el diseño de las cavidades de los EDFLs basados en resonadores de anillo de FO permite incrementar el ancho espectral de las señales generadas y lograr pulsos ultracortos de manera más eficiente, debido a que prescinden del uso de dispositivos de realimentación de la emisión estimulada que son de banda limitada $[28,29,84,120]$.

El diseño empleado en nuestro trabajo se basa en un conjunto de dos cavidades acopladas, una cavidad activa formada por un par de FGBs y una FO dopada con $\mathrm{Er}^{3+}$ y una segunda cavidad formada por un tramo de FO cortada adecuadamente para generar una reflexión de Fresnel en aire con una reflectividad próxima al $4 \%$, como se puede observar en nuestros trabajos $[2,46]$. Este sistema es compacto, implementeado mediante dispositivos discretos y muy flexible en el empleo de distinto tipo de FBGs y en la obtención de diversas salidas de pulsos cortos y frecuencias de repetición como podremos observar a lo largo de este capítulo y en el Capítulo 5, donde se muestra la implementación real del sistema láser.

\subsubsection{Propiedades espectroscópicas del $\mathrm{Er}^{3+}$}

Los láseres basados en FO dopada con $\mathrm{Er}^{3+}$ son de especial interés en este trabajo y operan en varias regiones del espectro óptico, en el rango de longitudes de onda desde el visible hasta el infrarrojo lejano (FIR). La región del espectro óptico próxima a los 1,55 m, la cual es empleada en los sistemas de comunicación ópticos de larga distancia, tiene un mayor atractivo debido a que la FO estándar presenta un muy bajo nivel de atenuación, como se discutió en el Capítulo 2. A primera vista, los EDFLs no parecen muy prometedores debido a que la transición ${ }^{4} I_{13 / 2} \rightarrow{ }^{4} I_{15 / 2}$ (relacionada con la emisión espontánea) termina en el estado fundamental del ion $\mathrm{Er}^{3+}$, como se puede observar en la Fig. 4.2. Sin embargo, una excitación adecuada en $980 \mathrm{~nm}$ permite obtener un sistema de tres o cuatro niveles, como se puede observar en las Figs. 4.2 (a) y (b), respectivamente, donde la transición 


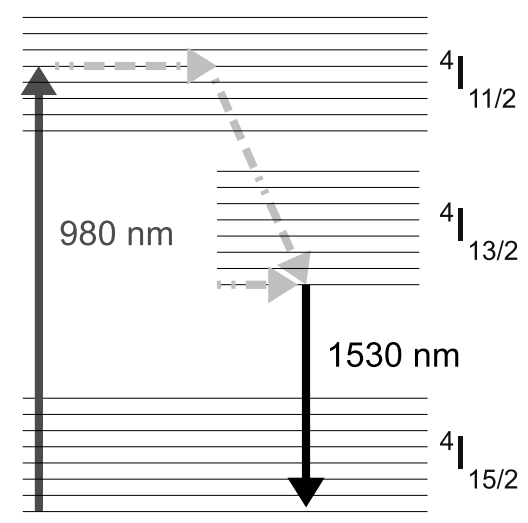

(a)

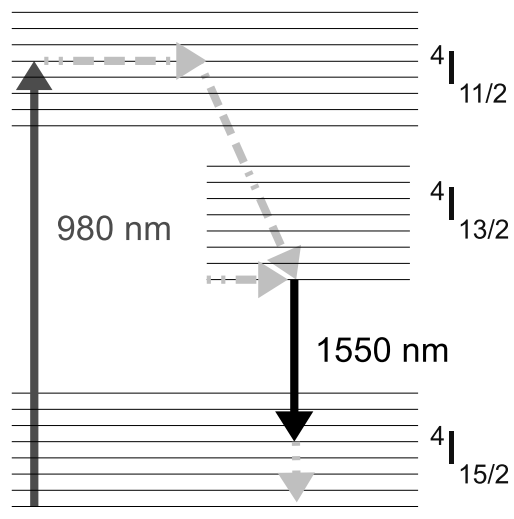

(b)

Figura 4.2: Diagrama simplificado de los niveles de energía del ion $\operatorname{Er}^{3+}$. La longitud de onda de excitación más importante corresponde a $980 \mathrm{~nm}$, mientras que la ASE se produce para longitudes de onda cercanas a los $1,53 \mu \mathrm{m}$. (a) La transición ${ }^{4} I_{11 / 2} \rightarrow{ }^{4} I_{13 / 2}$ es noradiativa. (b) Un sistema de cuatro niveles es también probable, en general para longitudes de onda mayor a 1,55 $\mathrm{mm}$, donde la emisión supera a las pérdidas por absorción.

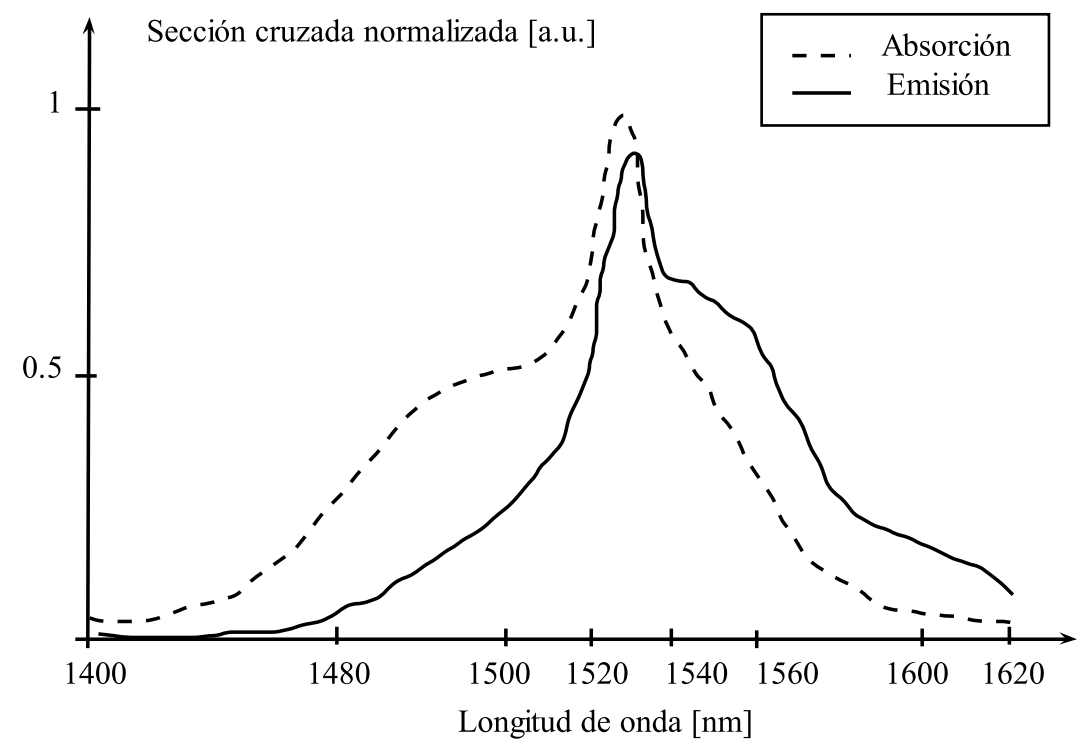

Figura 4.3: Medida de los espectros de absorción y la emisión normalizados del ion $\mathrm{Er}^{+3}$ de una FO dopada (LIEKKI Er30-4). Para $\lambda \approx 1,53 \mu \mathrm{m}$ la pérdida por absorción iguala a la emisión y en estado excitado se produce una ganancia. 
${ }^{4} I_{11 / 2} \rightarrow{ }^{4} I_{13 / 2}$ correspondiente a $2,75 \mu \mathrm{m}$ aproximadamente, se presenta como no-radiativa. Un sistema de cuatro niveles, también es probable en los mismos EDFLs, donde la emisión estimulada (para longitudes de onda mayor a la generada por la transición ${ }^{4} I_{13 / 2} \rightarrow{ }^{4} I_{15 / 2}$ ) se produce dentro del nivel fundamental, aunque alcanzando el estado no-excitado mediante otro proceso no-radiativo (en detrimento de la eficiencia de conversión). En general, ambos procesos, mostrados en las Figs. 4.2 (a) y (b), pueden funcionar en un mismo esquema láser, aunque su probabilidad de ocurrencia puede ser alterada mediante la elección de las características espectrales del sistema de realimentación. Para longitudes de onda de emisión próximas a la transición ${ }^{4} I_{13 / 2} \rightarrow{ }^{4} I_{15 / 2}$ (1,53 $\mu$ m aproximadamente), el esquema de tres niveles es más probable que el de cuatro niveles. De forma análoga, para una longitud de onda de emisión mayor a dicha transición, un sistema de cuatro niveles es más probable que uno de tres niveles. Un EDFL de tres niveles requiere que al menos la mitad de la población de iones deba ser elevado al estado excitado, por lo que presentan un umbral alto de excitación. En particular, en el EDFL analizado en este capítulo, un proceso de cuatro niveles presenta un umbral de excitación pequeño y es más probable debido a que la longitud de onda de las FBGs es próxima a 1,55 $\mu \mathrm{m}$.

Un tópico de importancia en la determinación de las características del medio de ganancia de un EDFL son los tiempos de vida media relacionados con los procesos de absorción y emisión el ion $\mathrm{Er}^{3+}$ en FOs dopadas. En general, el tiempo de vida de la transición ${ }^{4} I_{13 / 2} \rightarrow{ }^{4} I_{15 / 2}$ es alrededor de $10 \mathrm{~ms}$ y depende de los parámetros físicos de cada FO en particular, mientras que el tiempo de vida promedio del nivel ${ }^{4} I_{11 / 2}$ es varios órdenes de magnitud inferior a dicho valor (del orden de $20 \mu \mathrm{s}$ ). La excitación del medio de ganancia (FO dopada) mediante un haz de $980 \mathrm{~nm}$ envía población del nivel fundamental al ${ }^{4} I_{11 / 2}$, el cual decae en forma no-radiativa y en corto tiempo al ${ }^{4} I_{13 / 2}$. Por otro lado el ${ }^{4} I_{13 / 2}$ puede acumular población debida al bombeo hasta unos 10ms. Por lo mencionado, se puede considerar que toda la población fue transferida en ese tiempo directamente a dicho nivel. Si analizamos ahora el fundamental $\left({ }^{4} I_{15 / 2}\right)$, encontraremos que los niveles vibracionales superiores, correspondientes a transiciones en la banda de longitudes de onda largas) están prácticamente despoblados, permitiendo la operación del sistema bajo un esquema de 
cuatro niveles [14]. En general, el valor de la ganancia depende de los niveles de población ${ }^{4} I_{15 / 2}$ (fundamental) y ${ }^{4} I_{13 / 2}$, como

$$
g_{0}=\sigma_{e}(\lambda) N_{13 / 2}-\sigma_{a}(\lambda) N_{15 / 2}
$$

donde la $g_{0}$ es la ganancia del medio activo, y $N_{15 / 2}$ y $N_{13 / 2}$ dependen de la intensidad de excitación y los $\sigma_{e ; a}$ son probabilidades de los procesos mencionados, dependientes de la longitud de onda. En un sistema láser, en el umbral $g_{0}=l$ (ganancia igual a las pérdidas), donde ambos son valores positivos, mientras que la ganancia es generalmente $g_{0}>0$. En particular, la aplicación de este concepto en nuestro modelo analítico, el cual desarrollaremos a lo largo de este capítulo, se traduce en un factor de ganancia constante que depende exclusivamente del nivel de excitación (potencia óptica) aplicado. Esta aproximación es válida para todos los casos analizados (pulsos cortos del orden de los nanosegundos o menores de baja intensidad y alta frecuencia), y en general se aplica a la mayoría de los sistemas láser que operan en régimen mode locking [32].

Una transición importante $\left({ }^{4} S_{3 / 2} \rightarrow{ }^{4} I_{15 / 2}\right)$, relacionada a las pérdidas por scattering en el material, es generada para la zona espectral del verde, próxima a $550 \mathrm{~nm}$. Esta transición se produce mediante procesos de up conversion en FOs altamente dopadas y con altos niveles de excitación (aún para longitudes de onda de $980 \mathrm{~nm}$ y 1,48 $\mu \mathrm{m}$ ), de una forma similar a la emisión generada en FOs dopadas con $\mathrm{Yb}^{3+}$, como hemos podido observar en un trabajo previo [45].

Las longitudes de onda de $980 \mathrm{~nm}$ y 1,48 $\mu \mathrm{m}$ son comúnmente empleadas para bombear a las FOs dopadas con $\mathrm{Er}^{3+}$. El rendimiento de EDFLs mejora considerablemente cuando se emplean ambas longitudes de onda de excitación, debido a la ausencia de absorción en estado excitado. En efecto, los láseres semiconductores comerciales que operan en estas longitudes de onda se han desarrollado con el único propósito de generar medios de excitación para las FOs dopadas con $\mathrm{Er}^{3+}$. La ventaja de su implementación radica en que la emisión espontánea supera a la absorción en una región del espectro cercana a los 1,53 ㅆm, como se puede observar de la Fig. 4.3 para una FO dopada con $\mathrm{Er}^{3+}$ (altamente dopada), mien- 
tras que el ancho espectral donde se produce el proceso de emisión espontánea amplificada (ASE) suele ser del orden de las decenas de nanometros. Esta característica es de gran interés en el diseño de láseres de pulsos ultracortos en régimen de operación mode locking, debido a que la suma coherente de diversas componentes espectrales se puede extender en un amplio rango de frecuencias. Esta propiedad también es de importancia en el diseño de amplificadores ópticos para señales WDM para las bandas C y L del espectro óptico. La elección entre 980 y 1,48 $\mu \mathrm{m}$ no siempre es clara, ya que cada longitud de onda de bombeo tiene sus propios méritos, por lo que se emplean ambas de forma conjunta para desarrollar EDFLs con excelentes características de rendimiento [121] [122] [123].

\subsubsection{Régimen de operación mode locking}

En general, existen diversos modos de operación de los sistemas láser dependiendo de sus características constructivas $[15,113]$. Entre los modos de operación más importantes se encuentran: i) modo de onda continua o CW, donde el medio activo es continuamente excitado en un modo de resonancia simple (operación de una única frecuencia) o sobre múltiples modos; ii) modo cuasi-continuo donde la fuente de excitación del medio activo es conmutada en intervalos de tiempo cortos para prevenir el excesivo incremento de la temperatura (generada por las pérdidas por scattering y otros mecanismos cuánticos producidos dentro del láser); iii) el modo de operación de la ganancia conmutada, para el cual la excitación es modulada a intervalos muy cortos de tiempo (menores al tiempo de vida promedio de un fotón dentro de la cavidad) con el fin de obtener una emisión de pulsos cortos; iv) modo de operación $Q$-switch donde las pérdidas dentro de la cavidad son moduladas en tiempos más largos (del orden de los nanosegundos con frecuencias de repetición del orden del kilohertz y hasta algunas decenas megahertz), obteniendo láseres de pulsos energéticos mediante un bombeo continuo o pulsado; v) régimen de operación mode locking, iniciado y estabilizado mediante un modulador óptico, denominado active mode locking, o bien mediante un absorbente saturable (material o medio no-lineal) que genera varios pulsos cortos o ultracortos mediante una excitación continua. En otras ocasiones, los 
láseres exhiben oscilaciones o fluctuaciones caóticas, las cuales son muchas veces relacionadas a procesos denominados como self-pulsing [124,125], o simplemente presentan una salida mediante el proceso de emisión espontánea amplificada ASE (no hay emisión láser). Láseres que operan en régimen mode locking también son diseñados mediante un proceso de bombeo cuasi-continuo, y/o de forma conjunta con el régimen $Q$-switch dependiendo de las intensidades de excitación empleadas, como explica en su trabajo Hideur et.al. [28] para un láser basado en un medio activo de FO dopada con $\mathrm{Yb}^{3+}$. En general, con láseres mode locking (activos y/o pasivos, o bien que poseen o no una modulación externa de las pérdidas) se pueden generar trenes de pulsos con anchos temporales del orden de los picosegundos o menores, con frecuencias de repetición desde algunos cientos de megahertz hasta los gigahertz y energías de pulso moderadas (típicamente entre $10^{-9}-10^{-12} \mathrm{~J}$ ). El sistema analizado en la próxima sección es un láser de dos cavidades de FO acopladas mediante una FBG y que opera bajo el régimen mode locking de la siguiente manera. La cavidad activa del sistema es formada por una FO dopada con $\mathrm{Er}^{3+}$ y un par de FBG uniformes que sirven como dispositivos de realimentación de la emisión láser. Esta cavidad impone modos equiespaciados en frecuencia cada $\Delta f_{M}=c /\left(2 n_{e f f} L_{M}\right)$. La cavidad auxiliar o secundaria es acoplada a la cavidad activa mediante una de sus FBGs, estableciendo una distribución de frecuencias equiespaciadas en $\Delta f_{A}=c /\left(2 n_{e f f} L_{A}\right)$ a través de una reflexión de Fresnel (interfaz aire-FO). Ambas separaciones espectrales se corresponden con los modos longitudinales definidos para una cavidad Fabry-Perot, como hemos definido en la Ec. 4.1 y en el desarrollo del Capítulo 3. El acople o superposición coherente de estas componentes espectrales se produce sobre la FBG que es común a ambas cavidades, mientras que la eficiencia del proceso mode locking queda determinada por las características del medio activo y el sistema de realimentación, como se muestra en [32]. En general, la señal óptica generada bajo este régimen de operación se puede expresar a través de una suma finita de dichas componentes, similar a una representación por serie de Fourier como

$$
E_{\text {laser }}(t)=\sum_{n=1}^{N} E_{n} e^{i 2 \pi f_{n} t+i \phi_{n}}
$$


donde $E_{n}=\left|E_{n}\right| \exp \left(i \phi_{n}\right)$ es la envolvente compleja de la $n$-ésima componente espectral de frecuencia $f_{n}$, la cual presenta una fase $\phi_{n}$, en principio de naturaleza aleatoria, como muestra en su tesis de láseres mode locking Hudson [126, 127].

Bajo esta representación simplificada, el modelo de Hudson expresa un campo eléctrico a la salida del láser $E_{\text {laser }}(t)$ en función de un número finito de componentes espectrales $N$ y se supone que las fases aleatorias $\phi_{n}$ no guarda correlación alguna entre ellas. De forma contraria a esta descripción, las componentes espectrales amplificadas dentro del medio activo presentan un alto grado de coherencia, como hemos mencionado en las propiedades del mismo. Por lo tanto, la condición de ajuste de dichas componentes de fase en régimen mode locking, aplicada particularmente a nuestro sistema láser de dos cavidades acopladas, se alcanza fácilmente mediante una relación de números enteros de $\Delta f_{M} / \Delta f_{A}=N / M=$ $K^{-1}$, como se muestra en $[1,128]$.

En resumen, el modelo definido por la Ec. 4.4 es demasiado simplista y no explicita la relación entre los términos de amplitud y fase de cada una de las componentes espectrales con los parámetros físicos de un sistema láser real. De esta manera, la distorsión lineal y no-lineal de los campos elétricos propagados por una FO real es vagamente representada por dicho modelo. Recordando los análisis de propagación realizados en el Capítulo 2, la dispersión de segundo orden juega un rol importante en la determinación de las características temporales introduciendo también un chirp (inducido en las distintas componentes espectrales), mientras que el proceso no-lineal de SPM produce una distorsión de la fase temporal generando un ensanchamiento de las componentes espectrales (alterando, en su conjunto, la forma de los pulsos en el dominio del tiempo y la frecuencia). Por lo tanto, ambos procesos son de gran importancia en el modelado de estos sistemas láser, los cuales presentan una solución de tipo solitónica $[32,53,54]$. Una manera de resolver el problema del modelado de estos sistemas es aplicando un análisis de la propagación de los pulsos dentro de ambas cavidades del láser, como podremos observar en el desarrollo de nuestro modelo analítico y numérico, el cual permite un ajuste adecuado de los resultados teórico-experimentales $[2,46]$. 


\subsection{Descripción del modelo teórico}

Los métodos analíticos empleados para calcular la envolvente del campo eléctrico de salida de un láser, que opera bajo el régimen mode locking, dependen del grado de aproximación requerido por los resultados. Generalmente, el campo eléctrico es calculado mediante el uso de una "ecuación maestra" que depende de parámetros simples como: factores de pérdida y/o ganancia, dispersión y no-linealidad de las fibras ópticas empleadas en el sistema, y los coeficientes de reflexión o transmisión de los elementos de realimentación (filtros y/o espejos), como muestra en sus trabajos Haus et al. [31,32]. Sin embargo, en algunos casos este modelo no puede ser aplicado adecuadamente, en particular cuando las señales de luz propagadas dentro del sistema presentan un ancho de banda grande, por ejemplo, en el caso de los láseres de pulsos ultracortos y/o en señales generadas mediante peines de frecuencia ópticos por procesos de FWM [33-35]. El modelado de estos sistemas involucra diversos procesos de propagación lineales y no-lineales dentro de las cavidades del láser, una marcada dependencia de las características de los dispositivos con la longitud de onda, cambios en la velocidad de grupo y la aparición de otros procesos no-lineales relacionados al enganche coherente de las componentes espectrales de la señal generada.

En esta sección se presenta un modelo que permite calcular la salida de un láser de FO con dos cavidades y que opera en régimen APM, como se puede observar en el esquema de la Fig. $4.4[2,46]$. La cavidad principal (medio de ganancia) se construye con FO dopada con $\mathrm{Er}^{3+}$, mientras que la cavidad auxiliar queda definida por un tramo de FO estándar cortada en uno de sus extremos (perpendicular al eje de longitudinal de la FO), generando una reflexión de Fresnel del orden de $4 \%$. Las cavidades del láser están acopladas mediante una FBG común a ambas, mientras que el conjunto de las FBGs que conforman la cavidad principal proveen la realimentación de la emisión estimulada generada dentro del medio de ganancia.

Como mencionamos en el Capítulo 2, la propagación de la envolvente del campo eléctrico por una FO es analizada mediante la NLSE [16]. Suponiendo que es posible expresar dicha ecuación mediante un operador simplificado, cuyos coeficientes son integrados sobre la 


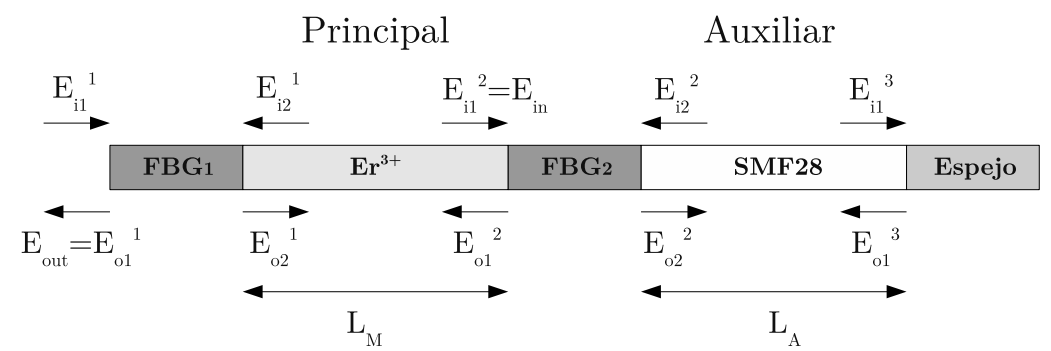

Figura 4.4: Esquema del láser de FO de dos cavidades acopladas mediante una FBG. La cavidad activa está definida por dos FBGs y una FO dopada con $\mathrm{Er}^{3+}$, mientras que la cavidad auxiliar, acoplada a la anterior por medio de una FBG común a ambas está construida con un tramo de FO estándar cuyo extremo restante es cortado de forma adecuada para obtener un dispositivo de baja reflectividad (reflexión de Fresnel). El sistema opera en régimen APM cuando se verifica la condición de ajuste de la fase, definida por la Ec. 4.8.

longitud de propagación [22,23], o sea en $z=L$, y asumiendo que el error de realizar dicha aproximación es despreciable para pequeños incrementos de longitud $\delta L$, la misma se puede expresar como

$$
\begin{gathered}
\hat{O}_{m}(\cdot)=i \frac{g L_{M}}{2}(\cdot)+\frac{\beta_{2_{m}} L_{M}}{2} \frac{\partial^{2}(\cdot)}{\partial t^{2}}-\gamma_{m} L_{M}|(\cdot)|^{2}(\cdot), \\
\hat{O}_{a}(\cdot)=i \frac{\alpha_{a} L_{A}}{2}(\cdot)+\frac{\beta_{2_{a}} L_{A}}{2} \frac{\partial^{2}(\cdot)}{\partial t^{2}}-\gamma_{a} L_{A}|(\cdot)|^{2}(\cdot),
\end{gathered}
$$

donde $\hat{O}_{m}(\cdot)$ y $\hat{O}_{a}(\cdot)$ son los operadores de las cavidades principal y auxiliar, respectivamente. El coeficiente de atenuación para una FO estándar $\alpha_{a}$ es reemplazado por la ganancia del medio activo $g$ en el operador de la cavidad principal. Los coeficientes de dispersión de segundo orden también son diferentes, para FO estándar es $\beta_{2_{a}}$ y en FO dopada queda definido mediante $\beta_{2_{m}}$. De la misma manera debemos definir dos parámetros no-lineales $\gamma_{m}$ y $\gamma_{a}$ en cada una de las cavidades del láser. Todos los parámetros mencionados presentan una dependencia con la longitud de onda. Por lo tanto, es importante determinar la longitud de onda de trabajo $\lambda_{0}$ para no cometer errores sistemáticos adicionales. El cálculo mediante las Ecs. 4.5 y 4.6 se realiza aplicado a un campo eléctrico $E(z=0, t)$ a través de $(\cdot)$ para obtener un campo de salida $E(z=\delta L, t)$. En el análisis siguiente, las variables in- 
dependientes del tiempo $t$ y la longitud de propagación $z$ son despreciadas para simplificar la notación.

Las características de reflexión y transmisión de las FBGs (en amplitud y fase) pueden ser calculadas mediante el TMM, como se mostró en el Capítulo 3. Sin embargo, el acoplamiento de los campos eléctricos provenientes de ambas cavidades sobre la $\mathrm{FBG}_{2}$, también puede ser aproximado mediante el uso de una matriz unitaria cuyos elementos están relacionados a los coeficientes de reflexión y transmisión de la FBG [23], de forma similar a una matriz de scattering [56]. Luego, el campo eléctrico de salida es obtenido mediante el uso del coeficiente de transmisión de la $\mathrm{FBG}_{1}$, sobre el campo resultante del viaje de ida y vuelta producido en la cavidad activa. De esta manera, el acoplamiento de los campos que entran y salen de las FBGs está definido por una matriz cuadrada $U$, donde $E_{i k}$ y $E_{o k}$ son los campos eléctricos de entrada/salida, respectivamente, los cuales se definen mediante los elementos $U_{m n}$ de la matriz $U(\operatorname{con} m ; n=1,2$.) y donde $k=1,2$. representa la ubicación de dichos campos en cada dispositivo, o sea

$$
\left[\begin{array}{l}
E_{o 1} \\
E_{o 2}
\end{array}\right]=\left[\begin{array}{ll}
U_{11} & U_{12} \\
U_{21} & U_{22}
\end{array}\right]\left[\begin{array}{l}
E_{i 1} \\
E_{i 2}
\end{array}\right] .
$$

Los elementos $U_{m n}$ dependen de los parámetros constructivos de las FBGs. En general, los elementos de la diagonal principal de la matriz $U$ se pueden expresar como función del coeficiente de reflexión $\rho(\omega)$, mientras que los elementos de la diagonal secundaria se pueden escribir en términos del coeficiente de transmisión $\tau(\omega)$, como veremos en la próxima sección. En general, el acoplamiento de los campos entrada/salida sobre la $\mathrm{FBG}_{2}$ queda definido por la Ec. 4.7. Por lo tanto, el acoplamiento coherente de los campos eléctricos (o pulsos), provenientes de ambas cavidades del láser, depende de su intensidad y de la fase, la cual debe cumplir la siguiente condición de ajuste, dada por

$$
\frac{L_{M}}{L_{A}}=\frac{N}{M}=K
$$

donde $L_{M}$ y $L_{A}$ son las longitudes físicas de FO de las cavidades principal y auxiliar, respectivamente, y $K$ expresa un número entero o bien racional compuesto de una fracción 
de números primos o coprimos [1]. En estos casos, el sistema produce un acoplamiento de dos señales coherentes provenientes de ambas cavidades del láser, resultando en una señal estable. Esta señal es estacionaria, de potencia uniforme, pulsada y periódica, con un período temporal bien definido. De esta manera, el proceso de enganche de los modos de forma coherente, que establece las propiedades de dicha señal, es determinado por las características del medio activo y por las propiedades lineales (atenuación y/o ganancia, y dispersión de segundo orden) y no-lineales (efecto Kerr o SPM) de las FOs utilizadas.

En este sentido, empleando la Ec. 4.7, se puede definir el modelo analítico que describe la propagación en ambas cavidades del láser, mediante un sistema de ecuaciones no-lineales acopladas como

$$
\begin{gathered}
e^{-i \phi_{m}} \hat{O_{m}} U_{22_{1}} \hat{O_{m}} E_{o 1}=E_{i 1} \\
e^{i \phi_{a}} \hat{O_{a}} U_{11_{3}} \hat{O_{a}} E_{o 2}=E_{i 2},
\end{gathered}
$$

donde el subíndice $p=1,2$ y 3 de $U_{m n_{p}}$ representa cada uno de los dispositivos del sistema, y los operadores que describen la propagación en las cavidades principal y auxiliar se expresan mediante los subíndices $m$ y $a$, respectivamente. Los factores de fase $\phi_{m}$ y $\phi_{a}$ están relacionados con las longitudes de camino óptico de las cavidades principal y auxiliar, respectivamente, de la siguiente manera

$$
\phi_{m, a}=2 \pi L_{M, A} n_{e f f} / \lambda_{0}
$$

donde $\lambda_{0}$ es la longitud de onda de trabajo en el vacío.

Reescribiendo los campos de salida $E_{\text {ok }}$ de las Ecs. 4.9 y 4.10 mediante la Ec. 4.7, expresándolos en función de los campos de entrada $E_{i k}$ y reescribiendo las ecuaciones del sistema se obtiene

$$
\begin{gathered}
e^{-i \phi_{m}} \hat{O_{m}} U_{22_{1}} \hat{O_{m}} U_{11_{2}} E_{i 1}-E_{i 1}+e^{-i \phi_{m}} \hat{O_{m}} U_{22_{1}} \hat{O_{m}} U_{12_{2}} E_{i 2}=0 \\
e^{i \phi_{a}} \hat{O_{a}} U_{11_{3}} \hat{O_{a}} U_{21_{2}} E_{i 1}+e^{i \phi_{a}} \hat{O_{a}} U_{11_{3}} \hat{O_{a}} U_{22_{2}} E_{i 2}-E_{i 2}=0,
\end{gathered}
$$


donde, de forma análoga a la aproximación SVEA supuesta generalmente en los cálculos de la propagación [4] [16], y específicamente en el modelado de este tipo de láseres de FO mediante una "ecuación maestra" definida por Haus et.al. [31] [32] se obtiene una solución estacionaria cuando los cambios en la envolvente de campo eléctrico resultante del cálculo de la propagación en ambas cavidades son pequeños, o sea

$$
\begin{gathered}
e^{-i \phi_{m}} \hat{O_{m}} U_{22_{1}} \hat{O_{m}} U_{11_{2}} E_{i 1}-E_{i 1} \longrightarrow 0 \\
e^{i \phi_{a}} \hat{O_{a}} U_{11_{3}} \hat{O_{a}} U_{22_{2}} E_{i 2}-E_{i 2} \longrightarrow 0
\end{gathered}
$$

De esta manera, suponiendo una representación matricial del sistema de ecuaciones nolineales acopladas, se debe cumplir que la diagonal secundaria compuesta por los operadores de la propagación (NLSE y elementos de la matriz de scattering de cada uno de los dispositivos del láser), debe ser nula, o sea

$$
\begin{gathered}
e^{-i \phi_{m}} \hat{O_{m}} U_{22_{1}} \hat{O_{m}} U_{12} E_{i 2}=0 \\
e^{i \phi_{a}} \hat{O_{a}} U_{11_{3}} \hat{O_{a}} U_{21_{2}} E_{i 1}=0 .
\end{gathered}
$$

Finalmente, aplicando las aproximaciones realizadas y operando de forma algebraica es posible escribir un par de ecuaciones que resultan como solución de las Ecs. 4.12 y 4.13 mediante

$$
\begin{aligned}
& \left(e^{i \phi_{a}} \hat{O_{a}} U_{11_{3}} \hat{O_{a}} U_{22_{2}}-e^{-i \phi_{m}} \hat{O_{m}} U_{22_{1}} \hat{O_{m}} U_{12_{2}}-1\right) E_{i 2}=0 \\
& \left(e^{-i \phi_{m}} \hat{O_{m}} U_{22_{1}} \hat{O_{m}} U_{11_{2}}-e^{i \phi_{a}} \hat{O_{a}} U_{11_{3}} \hat{O_{a}} U_{21_{2}}-1\right) E_{i 1}=0 .
\end{aligned}
$$

Como se puede observar, las Ecs. 4.18 y 4.19 permiten calcular las envolventes de campo eléctrico resultantes en ambas cavidades del sistema. El campo eléctrico de salida se puede obtener de dos maneras diferentes: i) mediante la aplicación del operador de la reflexión de la $\mathrm{FBG}_{2}$ sobre el campo $E_{i 1}$, luego aplicando el operador de la cavidad activa y obteniendo la salida mediante el operador de la transmisión de la $\mathrm{FBG}_{1}$; o bien, ii) por la difracción del espejo de la cavidad auxiliar, de forma análoga al caso anterior pero para el campo $E_{i 2}$, propagado en la cavidad auxiliar del sistema. 


\subsubsection{Análisis de la ecuación del sistema}

Suponiendo que las características espectrales de reflexión y/o transmisión de las FBG pueden ser escritas en términos de ecuaciones diferenciales lineales [23], la matriz $U$ que define el acoplamiento de los campos eléctricos concurrentes en una FBG queda definida mediante los siguientes operadores expresados en el dominio del tiempo como

$$
\begin{gathered}
U_{11}=R^{1 / 2}\left((\cdot)+\left(\frac{1}{\Omega^{2}}+i D\right) \frac{\partial^{2}(\cdot)}{\partial t^{2}}\right) \\
U_{12}=U_{21} \approx e^{i \phi}(1-R)^{1 / 2}\left((\cdot)-\frac{2 R}{(1-R) \Omega^{2}} \frac{\partial^{2}(\cdot)}{\partial t^{2}}\right) \\
U_{22}=i e^{-i 2 \phi} U_{11}^{*}=i e^{-i 2 \phi} R^{1 / 2}\left((\cdot)+\left(\frac{1}{\Omega^{2}}-i D\right) \frac{\partial^{2}(\cdot)}{\partial t^{2}}\right),
\end{gathered}
$$

donde los elementos se han aproximado mediante las constantes de la reflectividad $R=$ $\left|\rho\left(\omega_{\text {Bragg }}\right)\right|^{2}$ de las FBGs, la dispersión $D=d G D(\omega) /\left.d \omega\right|_{\omega=\omega_{\text {Bragg }}}$ (donde $G D(\omega)$ es el retardo de grupo de la FBG en función de la frecuencia angular $\omega$ ), el ancho de banda $\Omega=2 \pi c \Delta \lambda_{F B G} / \lambda_{B r a g g}^{2}$ expresado en unidades de la frecuencia angular y la fase $\phi=$ $\phi_{\rho}\left(\omega_{\text {Bragg }}\right)$ es la fase del coeficiente de reflexión de la FBG evaluada a la frecuencia angular correspondiente a la longitud de onda de Bragg, o sea $\omega_{\text {Bragg }}=2 \pi c / \lambda_{\text {Bragg }}$.

Suponiendo que las FBGs son idénticas, el semiespejo es considerado como un valor de reflectividad pequeño $\left(R_{3}=4 \%\right)$ en un ancho de banda grande comparado con el de las FBGs y que presenta una fase nula para todas las frecuencias evaluadas, las Ecs. 4.18 y 4.19 pueden ser escritas en términos de las aproximaciones realizadas para dichos dispositivos. Reemplazando cada uno de los elementos de las matrices, definidos para cada uno de los dispositivos empleados en el láser (FBGs y semiespejo), por las aproximaciones definidas en las Ecs. 4.20, 4.21 y 4.22, es posible establecer un estudio analítico del sistema de ecuaciones no-lineales. Empleando la Ec. 4.19 y las aproximaciones mencionadas se puede obtener

$$
\begin{gathered}
e^{-i \phi_{m}} \hat{O_{m}} U_{22_{1}} \hat{O_{m}}\left\{R_{2}^{1 / 2}\left(E_{i 1}+\left(\frac{1}{\Omega_{2}^{2}}+i D_{2}\right) E_{i 1}^{\prime \prime}\right)\right\} \\
-e^{i \phi_{a}} \hat{O_{a}} U_{11_{3}} \hat{O_{a}}\left\{e^{i \phi_{2}}\left(1-R_{2}\right)^{1 / 2}\left(1-\frac{2 R_{2}}{\left(1-R_{2}\right) \Omega_{2}^{2}} E_{i 1}^{\prime \prime}\right)\right\}-E_{i 1}=0,
\end{gathered}
$$


aplicando el operador $\hat{O_{m}}$ sobre el primer término se obtienen los campos en función de sus derivadas en el dominio del tiempo. Si nos quedamos con las derivadas de primer y segundo orden, y despreciando las derivadas de orden superior, resulta

$$
\begin{gathered}
e^{-i \phi_{m}} \hat{O_{m}} U_{22} R_{2}^{1 / 2}\left\{\left[\frac{i g}{2}-\gamma_{m}\left|R_{2}^{1 / 2}\left(E_{i 1}+\left(\frac{1}{\Omega_{2}^{2}}+i D_{2}\right) E_{i 1}^{\prime \prime}\right)\right|^{2}\right] \times\right. \\
\left.\left[E_{i 1}+\left(\frac{1}{\Omega_{2}^{2}}+i D_{2}\right) E_{i 1}^{\prime \prime}\right]+\frac{\beta_{2 m}}{2} E_{i 1}^{\prime \prime}\right\} .
\end{gathered}
$$

Además, la ganancia $g$ del sistema se satura rápidamente dependiendo de la intensidad de los pulsos generados. En nuestro modelo, la ganancia del medio activo se expresa como

$$
g=\frac{g_{0}}{1+I_{p} / I_{\text {sat }}}
$$

donde $g_{0}$ representa la ganancia de pequeña señal del medio activo para la longitud de onda de operación, y depende de la intensidad de bombeo o excitación aplicada y de los niveles de población de la FO dopada [14,32], $I_{p}=\int|E(t)|^{2} d t$ es la intensidad del pulso presente en el medio de ganancia, mientras que $I_{s a t}$ es la intensidad de saturación. Este último depende de las características del medio de ganancia y presenta generalmente un valor estándar expresado en unidades de potencia entre 5-15 dBm, como muestra Desurvire en su libro [14]. Luego, aplicando el operador de la reflectividad $U_{22_{1}}$ en la Ec. 4.2 .1 se obtiene

$$
\begin{gathered}
e^{-i \phi_{m}} \hat{O_{m}} i e^{-i 2 \phi_{1}} R_{1}^{1 / 2} R_{2}^{1 / 2}\left\{\left[\frac{i g}{2}-\gamma_{m}\left|R_{2}^{1 / 2}\left(E_{i 1}+\left(\frac{1}{\Omega_{2}^{2}}+i D_{2}\right) E_{i 1}^{\prime \prime}\right)\right|^{2}\right] \times\right. \\
{\left[E_{i 1}+\left(\frac{1}{\Omega_{2}^{2}}+i D_{2}\right) E_{i 1}^{\prime \prime}\right]+\frac{\beta_{2 m}}{2} E_{i 1}^{\prime \prime}+\left(\frac{1}{\Omega_{1}^{2}}-i D_{1}\right)\left[\left[\frac{i g}{2}-\gamma_{m}\left|R_{2}^{1 / 2}\left(E_{i 1}+\left(\frac{1}{\Omega_{2}^{2}}+i D_{2}\right) E_{i 1}^{\prime \prime}\right)\right|^{2}\right] E_{i 1}^{\prime \prime}\right.} \\
\left.\left.-2 \gamma_{m} R_{2}\left[\left(E_{i 1}^{\prime 2}+\left(E_{i 1}+\frac{1}{\Omega_{2}^{2}} E_{i 1}^{\prime \prime}\right) E_{i 1}^{\prime \prime}\right)\left(E_{i 1}+\left(\frac{1}{\Omega_{2}^{2}}+i D_{2}\right) E_{i 1}^{\prime \prime}\right)-2\left(E_{i 1}+\frac{1}{\Omega_{2}^{2}} E_{i 1}^{\prime \prime}\right) E_{i 1}^{\prime 2}\right]\right]\right\}
\end{gathered}
$$

Finalmente, aplicamos por segunda vez el operador de la cavidad principal $\hat{O_{m}}$, encontrando una aproximación para el primer término de la Ec. 4.19, el cual es definido como

$$
i e^{-i\left(\phi_{m}+2 \phi_{1}\right)} R_{1}^{1 / 2} R_{2}^{1 / 2}\left\{\left\{\frac{i g}{2}-\gamma_{m} \mid\left[\frac{i g}{2}-\gamma_{m}\left|R_{2}^{1 / 2}\left(E_{i 1}+\left(\frac{1}{\Omega_{2}^{2}}+i D_{2}\right) E_{i 1}^{\prime \prime}\right)\right|^{2}\right] \times\right.\right.
$$




$$
\begin{gathered}
{\left[E_{i 1}+\left(\frac{1}{\Omega_{2}^{2}}+i D_{2}\right) E_{i 1}^{\prime \prime}\right]+\frac{\beta_{2 m}}{2} E_{i 1}^{\prime \prime}+\left(\frac{1}{\Omega_{1}^{2}}-i D_{1}\right)\left[\left[\frac{i g}{2}-\gamma_{m}\left|R_{2}^{1 / 2}\left(E_{i 1}+\left(\frac{1}{\Omega_{2}^{2}}+i D_{2}\right) E_{i 1}^{\prime \prime}\right)\right|^{2}\right] E_{i 1}^{\prime \prime}\right.} \\
\left.\left.-2 \gamma_{m} R_{2}\left[\left(E_{i 1}^{\prime 2}+\left(E_{i 1}+\frac{1}{\Omega_{2}^{2}} E_{i 1}^{\prime \prime}\right) E_{i 1}^{\prime \prime}\right)\left(E_{i 1}+\left(\frac{1}{\Omega_{2}^{2}}+i D_{2}\right) E_{i 1}^{\prime \prime}\right)-2\left(E_{i 1}+\frac{1}{\Omega_{2}^{2}} E_{i 1}^{\prime \prime}\right) E_{i 1}^{\prime 2}\right]\right]\left.\right|^{2}\right\} \times \\
\left\{\left[\frac{i g}{2}-\gamma_{m}\left|R_{2}^{1 / 2}\left(E_{i 1}+\left(\frac{1}{\Omega_{2}^{2}}+i D_{2}\right) E_{i 1}^{\prime \prime}\right)\right|^{2}\right]\left[E_{i 1}+\left(\frac{1}{\Omega_{2}^{2}}+i D_{2}\right) E_{i 1}^{\prime \prime}\right]+\frac{\beta_{2 m}}{2} E_{i 1}^{\prime \prime}\right. \\
+\left(\frac{1}{\Omega_{1}^{2}}-i D_{1}\right)\left[\left[\frac{i g}{2}-\gamma_{m}\left|R_{2}^{1 / 2}\left(E_{i 1}+\left(\frac{1}{\Omega_{2}^{2}}+i D_{2}\right) E_{i 1}^{\prime \prime}\right)\right|^{2}\right] E_{i 1}^{\prime \prime}\right. \\
\left.\left.-2 \gamma_{m} R_{2}\left[\left(E_{i 1}^{\prime 2}+\left(E_{i 1}+\frac{E_{i 1}^{\prime \prime}}{\Omega_{2}^{2}}\right) E_{i 1}^{\prime \prime}\right)\left(E_{i 1}+\left(\frac{1}{\Omega_{2}^{2}}+i D_{2}\right) E_{i 1}^{\prime \prime}\right)-2\left(E_{i 1}+\frac{E_{i 1}^{\prime \prime}}{\Omega_{2}^{2}}\right) E_{i 1}^{\prime 2}\right]\right]\right\} \\
-\gamma_{m} \beta_{2 m} R_{2}\left[\left(E_{i 1}^{\prime 2}+\left(E_{i 1}+\frac{E_{i 1}^{\prime \prime}}{\Omega_{2}^{2}}\right) E_{i 1}^{\prime \prime}\right)\left(E_{i 1}+\left(\frac{1}{\Omega_{2}^{2}}+i D_{2}\right) E_{i 1}^{\prime \prime}\right)+\left(E_{i 1}+\frac{E_{i 1}^{\prime \prime}}{\Omega_{2}^{2}}\right) E_{i 1}^{\prime 2}\right. \\
\frac{i g}{4 \gamma_{m} R_{2}} E_{i 1}^{\prime \prime}+\left|\left(E_{i 1}+\left(\frac{1}{\Omega_{2}^{2}}+i D_{2}\right) E_{i 1}^{\prime \prime}\right)\right|^{2} E_{i 1}^{\prime \prime}+\left(\frac{1}{\Omega_{1}^{2}}-i D_{1}\right)\left(\left(\frac{1}{\Omega_{2}^{2}}+i D_{2}\right)\left(2+E_{i 1}^{\prime \prime}\right) E_{i 1}^{\prime \prime 3}\right. \\
\left.\left.\left.+2 E_{i 1} E_{i 1}^{\prime \prime 2}\left(2+E_{i 1}^{\prime \prime}\right)-3 E_{i 1}^{\prime 2} E_{i 1}^{\prime \prime}\right)\right]\right\} \cdot
\end{gathered}
$$

Como se puede observar, esta expresión es compleja y depende principalmente del campo eléctrico y sus derivadas de primer y segundo orden, de los parámetros lineales y no-lineales de la cavidad principal y de las FBGs que la componen. En general, una forma de establecer la condición de ajuste de la fase, definida para el primer término de la ecuación del sistema, se puede expresar como

$$
\Phi_{M}=\Phi_{L}+\Phi_{N L}=\left(-\left(\phi_{m}+2 \phi_{1}\right)+\phi_{D_{M}}\right)+\Phi_{N L_{M}}
$$

donde $\Phi_{L}$ representa el aporte de la fase lineal y $\Phi_{N L_{M}}$ el término de fase no-lineal inducido por SPM, mientras que el término de la fase lineal $\phi_{D_{M}}$ corresponde al aporte de la dispersión en el medio activo. Sin embargo, se debe notar que los términos de la fase lineal y no-lineal no son independientes uno del otro, debido a la naturaleza de los operadores definidos anteriormente. De esta forma se hace muy complicado obtener una condición de ajuste de la fase lineal y no-lineal total del sistema mediante el modelo planteado. Por otra 
parte, los términos de fase lineal de las FBGs (en este caso mediante $2 \phi_{1}$ ) y el término de fase $\phi_{m}$ aparecen de forma explicita e independientes de otros parámetros, lo cual facilita parcialmente el análisis.

El segundo término de la Ec. 4.2.1 se puede desarrollar despreciando las derivadas de orden superior y conservando las derivadas hasta de segundo orden. Operando de una forma similar a la anterior y aplicando el operador de la cavidad auxiliar $\hat{O}_{a}$, presente en el segundo término de dicha ecuación, se obtiene

$$
\begin{gathered}
e^{i \phi_{a}} \hat{O}_{a} U_{11_{3}}\left\{\left[\frac{i \alpha_{a}}{2}-\gamma_{a}\left(1-R_{2}\right)\left|\left(1-\frac{2 R_{2}}{\left(1-R_{2}\right) \Omega_{2}^{2}} E_{i 1}^{\prime \prime}\right)\right|^{2}\right] \times\right. \\
\left.\left(e^{i \phi_{2}} \sqrt{1-R_{2}}\left(1-\frac{2 R_{2}}{\left(1-R_{2}\right) \Omega_{2}^{2}} E_{i 1}^{\prime \prime}\right)\right)\right\},
\end{gathered}
$$

Luego, aplicando el operador $U_{11_{3}}=R_{3}$ (constante) a la ecuación anterior, y el segundo operador de la cavidad auxiliar $\hat{O}_{a}$, se obtiene

$$
\begin{gathered}
R_{3}\left\{\left[\frac{i \alpha_{a}}{2}-\gamma_{a}\left|\left[\frac{i \alpha_{a}}{2}-\gamma_{a}\left(1-R_{2}\right)\left|\left(1-\frac{2 R_{2}}{\left(1-R_{2}\right) \Omega_{2}^{2}} E_{i 1}^{\prime \prime}\right)\right|^{2}\right] e^{i \phi_{2}} \sqrt{1-R_{2}}\left(1-\frac{2 R_{2}}{\left(1-R_{2}\right) \Omega_{2}^{2}} E_{i 1}^{\prime \prime}\right)\right|^{2}\right] \times\right. \\
\left.\left[\left[\frac{i \alpha_{a}}{2}-\gamma_{a}\left(1-R_{2}\right)\left|\left(1-\frac{2 R_{2}}{\left(1-R_{2}\right) \Omega_{2}^{2}} E_{i 1}^{\prime \prime}\right)\right|^{2}\right] \sqrt{1-R_{2}}\left(1-\frac{2 R_{2}}{\left(1-R_{2}\right) \Omega_{2}^{2}} E_{i 1}^{\prime \prime}\right)\right]\right\} e^{i\left(\phi_{a}+\phi_{2}\right)}, \quad(4.30)
\end{gathered}
$$

donde el término relacionado a la dispersión de la $\mathrm{FBG}_{2}$ está ausente. En este caso es sencillo intuir que la condición de fase para la cavidad auxiliar queda definida por

$$
\Phi_{A}=\Phi_{L_{A}}+\Phi_{N L_{A}}=\left(\phi_{a}+\phi_{2}+\phi_{D_{A}}\right)+\Phi_{N L_{A}}
$$

donde $\Phi_{N L_{A}}$ depende principalmente de la no-linealidad de la cavidad auxiliar $\left(\gamma_{A} L_{A}\right)$, la atenuación, la reflectividad de la $\mathrm{FBG}_{2}$ y su ancho de banda. De todas formas, este término podría ser despreciado debido a que $R_{2}$ presenta generalmente un valor alto (> $90 \%$ ), manteniendo una potencia mayor en la cavidad activa del láser, mientras que el valor de $R_{3}$ es pequeño. La condición de ajuste de la fase impuesta por la Ec. 4.8, para valores de $K$ enteros, implica que la diferencia de las fases producidas en ambas cavidades debe verificar 


$$
\begin{aligned}
\Delta \Phi=\Phi_{M}-\Phi_{A}= & \left(-\left(\phi_{m}+2 \phi_{1}\right)+\phi_{D_{M}}\right)+\Phi_{N L_{M}} \\
& -\left(\phi_{a}+\phi_{2}+\phi_{D_{A}}\right)-\Phi_{N L_{A}}=0,
\end{aligned}
$$

donde la diferencia entre los términos de la fase lineal y no-lineal de ambas cavidades es compensada de alguna forma, mientras que es incrementado el valor de la fase relacionado a las FBGs. Una forma de establecer un criterio para resolver el problema de la incertidumbre de la fase, al menos de forma cualitativa, es mediante el planteo de un ejemplo numérico, como veremos a continuación.

\section{Resultados obtenidos mediante una herramienta de representación simbólica}

Eligiendo adecuadamente los parámetros del sistema, mediante estudio de los distintos componentes tales como las longitudes y parámetros de las FOs empleadas, las características de las FBGs y la ganancia contante del medio activo, se puede hallar una solución estacionaria suponiendo que el campo $E_{i 1}$ es un pulso del tipo secante hiperbólica de ancho $T_{0}=1$ ns y potencia $P_{0}=100 \mathrm{~mW}$ (potencia óptica dentro de la cavidad activa). En la Tabla 4.1 se pueden observar los parámetros principales del modelo aplicados en el siguiente calculo analítico, donde los valores de la dispersión de las FBGs son aproximadamente nulos. La Fig. 4.6 muestra la solución encontrada mediante el uso de una herramienta matemática de representación simbólica. La potencia de la envolvente del campo eléctrico resultante, calculada mediante el producto de la intensidad y el área efectiva de la FO, presenta un ancho temporal igual a 1,73 ns y un valor de la fase aproximadamente constante alrededor de pequeñas variaciones de $-\pi / 2$ radianes. La solución hallada podría ser concluyente si el sistema de ecuaciones no-lineales pudiera admitir diversos valores de dispersión de las FBGs. Sin embargo, no se encuentran resultados consistentes para valores de dispersión no nulos, lo cual implica que el modelo teórico es sensible a estas variaciones [46].

Para asegurar que las soluciones encontradas son representativas del sistema de ecuaciones, la hipótesis relacionada a la anulación de los términos de la diagonal secundaria del sistema de ecuaciones debe ser verificada. De esta forma, el primer término de la Ec. 4.13 que define uno de los operadores de la diagonal secundaria del sistema puede ser evaluado mediante 


\begin{tabular}{l|l|l} 
Cavidad principal & Cavidad auxiliar & FBGs \\
\hline$L_{M}=1,86 \times 10^{6} \lambda_{0} / n_{e f f}=1,976 \mathrm{~m}$ & $L_{A}=L_{M}$ & $\left|\rho_{1}\right|^{2}=\left|\rho_{2}\right|^{2}=0,75$ \\
$g_{0}=0,2735 \mathrm{~m}^{-1}$ & $\alpha_{a}=4 \times 10^{-5} \mathrm{~m}^{-1}$ & $\lambda_{1}=\lambda_{2}=1554 \mathrm{~nm}$ \\
$\beta_{2_{m}}=108 \mathrm{ps}^{2} / \mathrm{km}$ & $\beta_{2_{a}}=-20 \mathrm{ps}^{2} / \mathrm{km}$ & $\Delta \lambda_{1 ; 2}=0,2 \mathrm{~nm}$ \\
$\gamma_{m}=4,5745 \times 10^{-3} \mathrm{~W}^{-1} \mathrm{~m}^{-1}$ & $\gamma_{a}=1,6519 \times 10^{-3} \mathrm{~W}^{-1} \mathrm{~m}^{-1}$ & $D_{1 ; 2} \approx 0 \mathrm{ps} / \mathrm{nm}$
\end{tabular}

Tabla 4.1: Parámetros principales del sistema láser de cavidades acopladas que opera en régimen APM. Entre estos se destacan, por su importancia, los parámetros físicos de las FBGs y la dispersión y no-linealidad de la FO dopada con $\mathrm{Er}^{3+}$. La determinación de dichos valores es importante para lograr una correcta representación de la salida del láser mediante los modelos numéricos implementados.

la aplicación del campo de entrada $E_{i 1}$, resultando

$$
E_{\text {zero }}=e^{i \phi_{a}} \hat{O}_{a} U_{11_{3}} \hat{O}_{a} U_{21_{2}} E_{i 1}
$$

donde $E_{z e r o}$ es la envolvente de campo eléctrico resultante, como se observa en la Fig. 4.5. Se puede observar que la potencia óptica es varios órdenes de magnitud inferior a la encontrada en la Fig.4.6 (a), mientras que la fase presenta un valor que no es del todo despreciable (comparada con la respuesta observada en la Fig. 4.6 (b)). Estrictamente, el operador de la diagonal secundaria, relacionado al segundo término de la Ec. 4.12 también debe anularse. Esta verificación se realiza de una forma similar a la anterior mediante el uso del campo eléctrico resultante de la cavidad auxiliar $E_{i 2}$.

De todas formas, los resultados encontrados de forma experimental en [1], se verifican de forma adecuada mediante las simulaciones mostradas en la Fig. 4.6, para una relación entre las longitudes de ambas cavidades unitaria, o sea $K=1$. Estos resultados han sido publicados en nuestro estudio analítico [46]; sin embargo, en dicho trabajo los valores de la dispersión de las FBGs y el cálculo del término no-lineal son dos temas sensibles que deben ser abordados con cierto cuidado. Por un lado, el perfil de la dispersión de las FBGs depende de su posición espectral relativa (entre las FBGs de la cavidad principal), mientras 


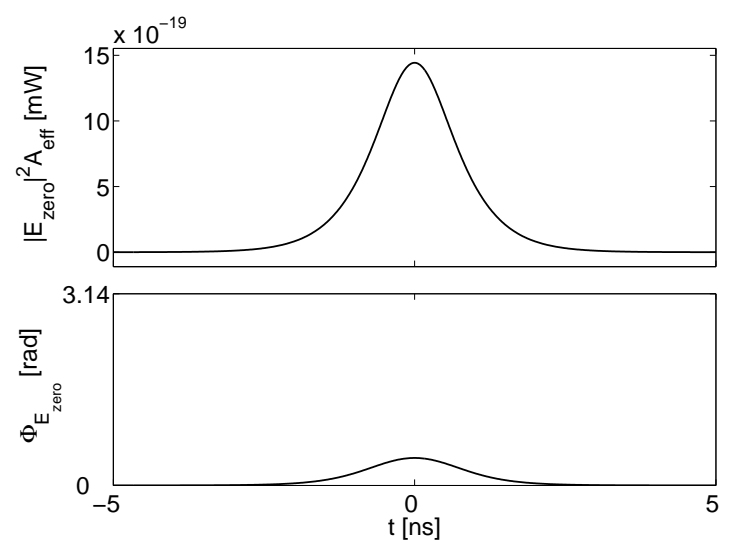

Figura 4.5: Potencia y fase del campo eléctrico resultante de la diagonal secundaria del sistema. El primer término de la Ec. 4.13 aplicado al campo $E_{i 1}$ tiende a ser nulo.

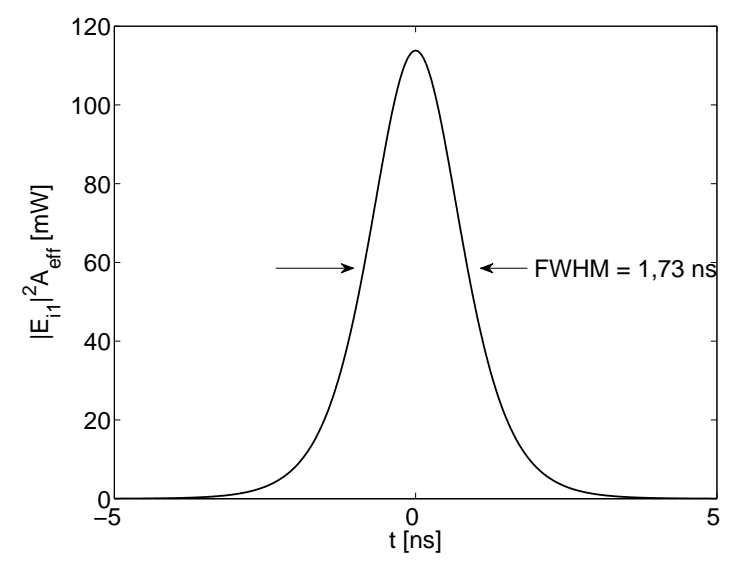

(a)

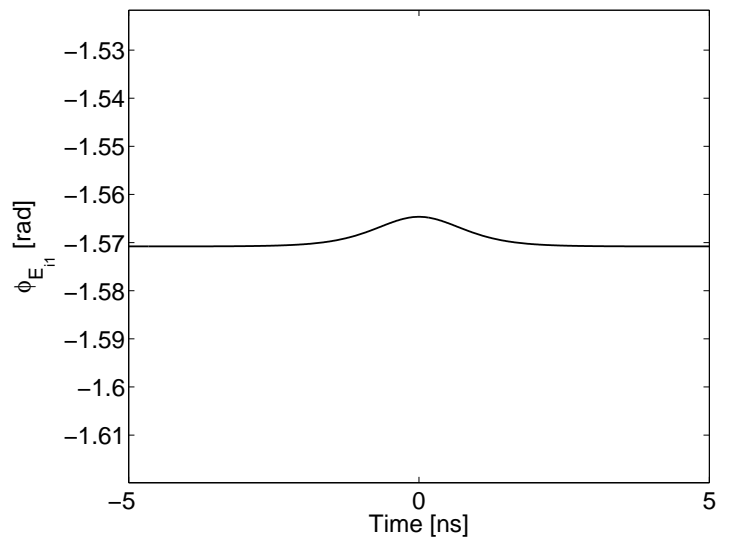

(b)

Figura 4.6: (a) Potencia del campo eléctrico $E_{i 1}$, solución de láser APM. (b) Fase de $E_{i 1}$ en función del tiempo.

que la dispersión resonante de la FO dopada con $\mathrm{Er}^{3+}$ puede cambiar en módulo y signo dependiendo de la longitud de onda de trabajo del láser (establecida en nuestro caso por las FBGs) [14]. Por otra parte la no-linealidades deberían ser evaluadas de forma conjunta con la dispersión, con el fin de obtener una solución del tipo solitónica, típica en sistemas láser que operan en régimen APM $[32,53]$, y de forma análoga a la descripción realizada 
en el Capítulo 2. Por lo tanto, esta solución no es del todo representativa del sistema, debido a que no admite cambios en el parámetro de dispersión cromática introducido por las FBGs. En general, esto sucede cuando las distintas derivadas de los campos eléctricos propagados son calculadas mediante aproximaciones numéricas simples, introduciendo errores sistemáticos adicionales. Una forma de resolver este problema consiste en emplear un modelo numérico que incluye el perfil de las FBGs (calculadas mediante el TMM), basado en el acoplamiento de los campos de forma coherente mediante una matriz de scattering, y que permite calcular la propagación de los campos eléctricos mediante el SSM de paso variable. De esta manera es posible limitar los errores y estimar correctamente el aporte de fase no-lineal, como veremos en la próxima sección.

\subsection{Modelo numérico implementado mediante SSM}

Los modelos numéricos empleados para calcular la propagación de la envolvente del campo eléctrico en un sistema láser están focalizados en la resolución de la NLSE [16]. Un método de Runge-Kutta es empleado para encontrar una solución en un sistema que presenta condiciones de contorno y parámetros simples [31,36]. En general, se requiere una representación completa (módulo y fase) de los elementos que conforman el sistema de realimentación, lo que produce un incremento del costo computacional. Una solución efectiva a este problema la brinda el SSM de paso variable, el cual permite calcular la propagación de los campos eléctricos en ambas cavidades del láser, definiendo el efecto conjunto de la no-linealidad y la dispersión de segundo orden y alcanzando soluciones muy precisas en tiempos cortos. Además, este método presenta una gran flexibilidad de integración con la matriz de scattering, la cual es empleada nuevamente para acoplar los campos eléctricos que concurren a una única FBG, como será mostrado a continuación.

Para analizar el modo de operación pulsado del láser esquematizado en la Fig. 4.4, se emplea nuevamente un pulso del tipo secante hiperbólica como semilla y la mayoría de los parámetros mencionados en la Tabla 4.1. Otros parámetros de interés como la longitud de onda de trabajo $\lambda_{0}=1553,55 \mathrm{~nm}$ (establecida por la longitud de onda de las FBGs), el 


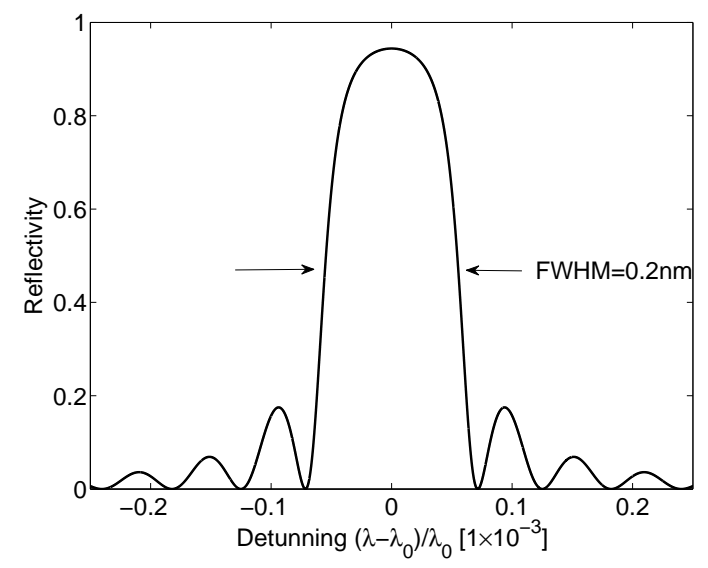

(a)

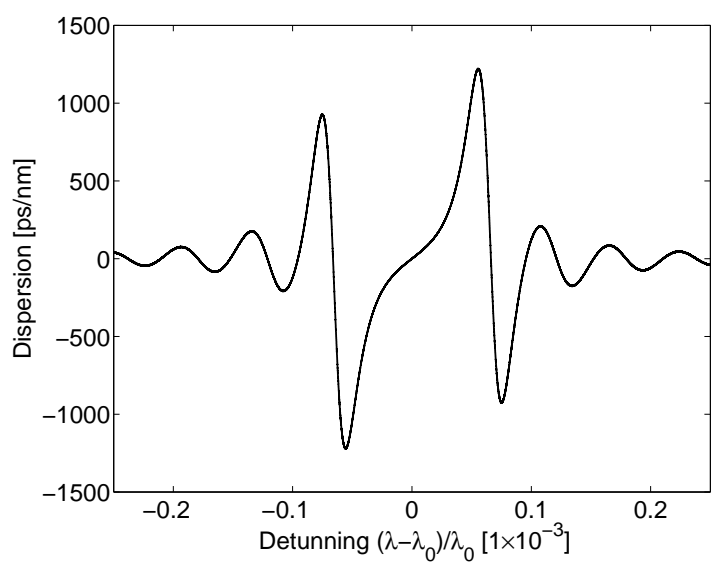

(b)

Figura 4.7: Características de las FBGs utilizadas en nuestro modelo numérico. (a) Reflectividad y (b) dispersión en función del detuning normalizado a la longitud de onda de $\operatorname{Bragg}\left(\left(\lambda-\lambda_{\text {Bragg }}\right) / \lambda_{\text {Bragg }}\right)$.

índice de refracción efectivo $n_{\text {eff }}=1,46$ y el tiempo de muestreo del campo eléctrico $T_{s}=$ 10 ps (utilizando alrededor de $2^{18}$ puntos), son importantes en la representación numérica del problema. Las FBGs empleadas en este caso presentan las siguientes características: $\left|\rho_{1}\right|^{2}=\left|\rho_{2}\right|^{2}=0,93, \lambda_{1}=\lambda_{2}=1553,55 \mathrm{~nm}$ y $\Delta \lambda_{1,2_{3 d B}}=0,2 \mathrm{~nm}$ y son modeladas como funciones dependientes de la longitud de onda, como la reflectividad y la transmitancia de las mismas. En nuestro trabajo [2], fueron medidas las características espectrales de las FBGs, tales como los valores de la reflectividad, ancho de banda y longitud de las mismas. Estos valores fueron empleados para modelar las características espectrales de dichos dispositivos mediante el TMM, como se puede observar en la Fig. 4.7. Es importante notar que el valor de la dispersión de las FBGs presenta una dependencia con la longitud de onda, pudiendo alterar los resultados de forma significativa.

En nuestro modelo numérico los operatores definidos por las Ecs. 4.5 y 4.5 se resuelven mediante el uso del SSM de paso variable, con el fin de calcular el término de la dispersión aplicado en el dominio transformado y el término de fase no-lineal en el dominio del tiempo, como se muestra en el Apéndice. Para calcular la propagación en ambas cavidades del 


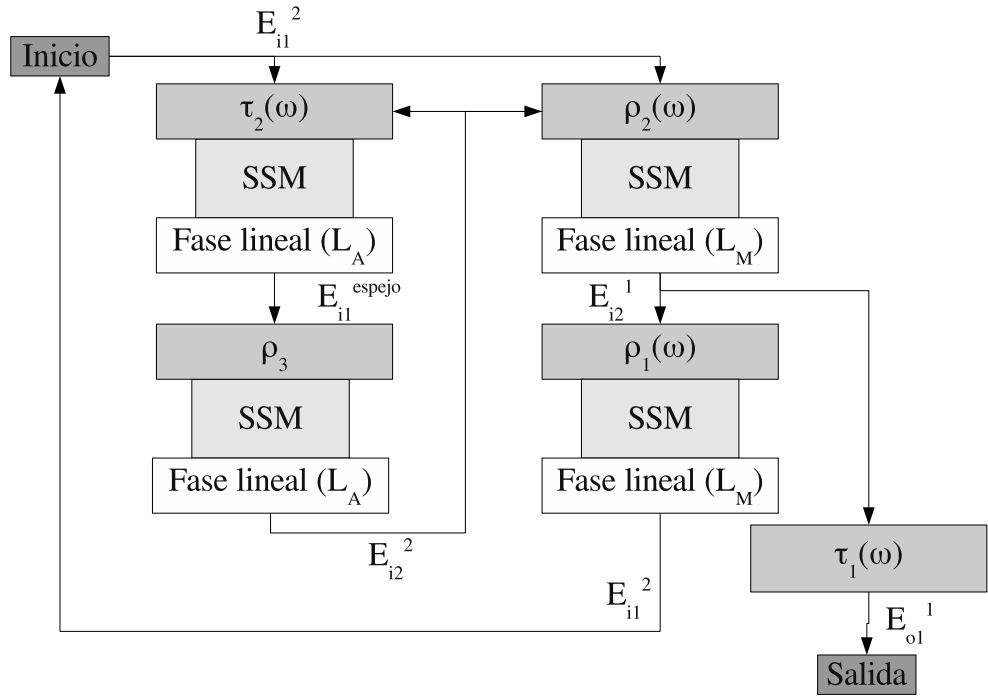

Figura 4.8: Diagrama de flujos del modelo numérico implementado para un láser APM pasivo de dos cavidades acopladas donde $L_{M}=L_{A}(K=1)$. Los campos provenientes de ambas ramas del diagrama deben ser sincronizados adecuadamente en tiempo y espacio para evitar errores sistemáticos en el cálculo de la salida del láser.

láser se aplica el SSM de paso variable para limitar los errores de fase a $1 \times 10^{-4} \mathrm{rad}$, con tolerancias $\left\|E_{s}-E_{s-1}\right\| /\left\|E_{s-1}\right\|<1 \%$ para $s=1, \ldots, S$ pasos, como Skin et al. define en [52] y, particularmente como considero en un trabajo de mi autoría, relacionado a la comparación de resultados numéricos y experimentales [2]. Dado que los valores de las reflectividades de las FBGs son altos (> $90 \%$ ), en este caso en particular, el tiempo de ida y vuelta es mucho menor que el tiempo de vida promedio de un fotón dentro de la cavidad activa del láser (del orden de 0,35 us). Este modelo permite analizar la interacción de los procesos lineales (atenuación, GVD y dispersión de las FBGs) y no-lineales (SPM y de autosaturación de la ganancia) relacionados con la propagación aproximada de un solitón dentro de las cavidades del láser y, además, es capaz de determinar la envolvente del campo eléctrico en otros puntos del sistema.

El modelo numérico funciona de la siguiente manera. En un principio, un campo eléctrico inicial $E_{i n}=E_{i 1}$ es usado como entrada, el cual es definido mediante un pulso tipo secante hiperbólica que es bien conocido en sistemas APM, como describen los trabajos de Martí- 


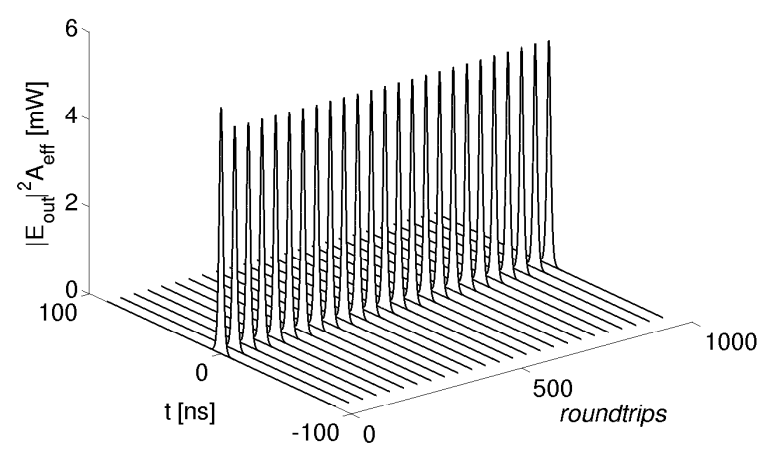

(a)

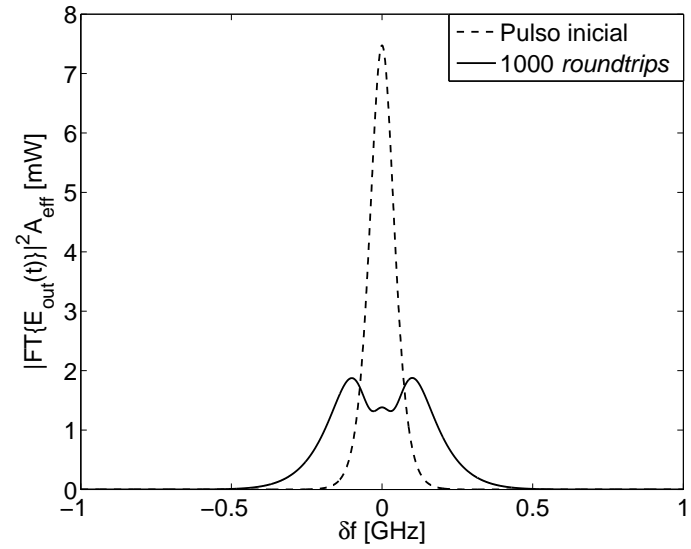

(b)

Figura 4.9: (a) Evolución temporal de la potencia del pulso de entrada para $K=1$. Las características del pulso propagado se mantienen durante más de 1000 roundtrips (equivalente a 20 ss aproximadamente), lo que permite establecer una solución estable. (b) Espectro del pulso de entrada y salida. El ensanchamiento espectral se debe al efecto del proceso no-lineal de SPM.

nez et al. $[53,54]$. En este instante la salida es nula y el pulso es propagado dentro de ambas cavidades hasta completar un viaje de ida y vuelta. Luego, estos campos son rápidamente actualizados de forma iterativa hasta alcanzar una solución estable, como veremos a continuación. El criterio elegido para determinar una solución adecuada (en módulo y fase) se basa en dos hipótesis: i) la potencia de los pulsos resulta uniforme para una condición de ajuste de láser, y ii) el ancho temporal de los pulsos propagados permanece inalterado para un número grande de viajes de ida y vuelta (tiempo de propagación grande). Ambas hipótesis se verifican para un apropiado conjunto de parámetros del sistema. De todas formas, debemos notar que para cada condición de ajuste establecida por la Ec. 4.8, el algoritmo implementado es ligeramente diferente. Específicamente, es necesario combinar los campos eléctricos que provienen de ambas cavidades mediante una adecuada sincronización espacial y temporal. Cuando las longitudes de las cavidades del láser son iguales, o bien $K=1$, el campo eléctrico de entrada $E_{i n}$ visto en la Fig. 4.4 es transmitido hacia 


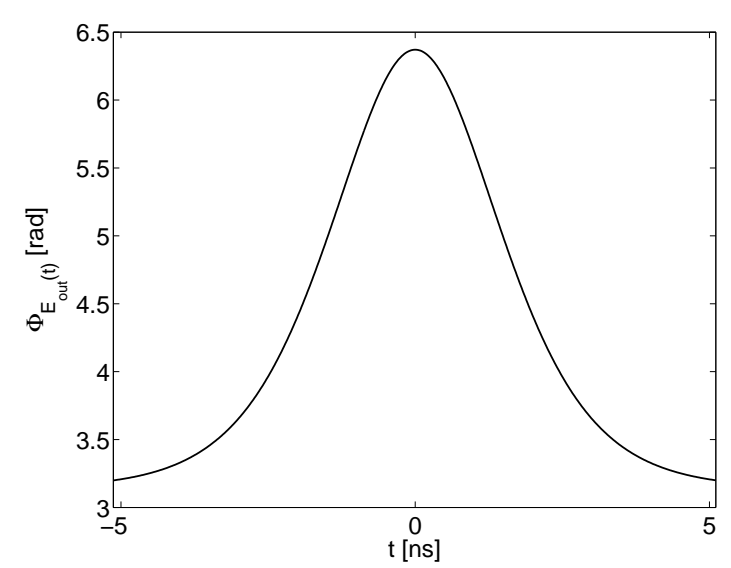

(a)

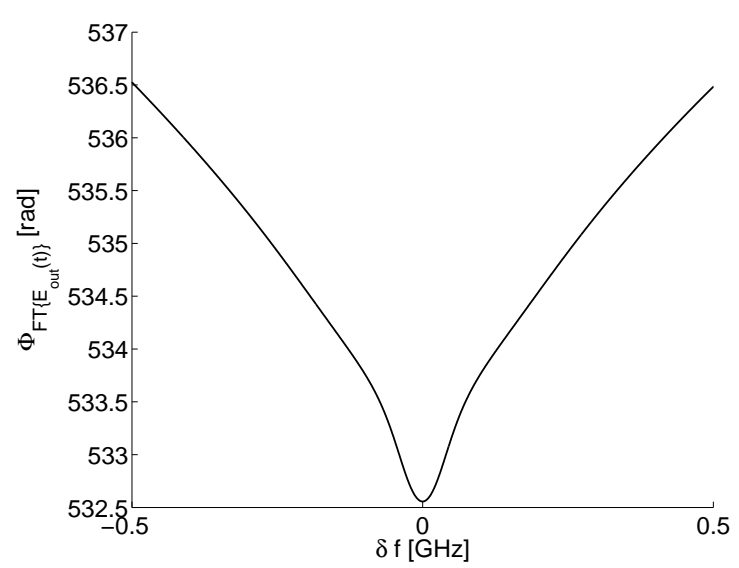

(b)

Figura 4.10: (a) Fase del pulso en el dominio del tiempo. (b) Fase de la transformada de Fourier del pulso de salida.

la cavidad auxiliar y, en el mismo instante, es reflejado y propagado dentro de la cavidad principal del láser. Los campos resultantes en la $F_{B G}$ y el espejo (reflexión al final de la FO) son nuevamente reflejados hacia la $\mathrm{FBG}_{2}$, completando a viaje de ida y vuelta. El diagrama de flujos correspondiente a esta descripción del modelo puede ser visto en la Fig. 4.8. El resultado de esta propagación puede ser visto en la evolución de la potencia del pulso en el dominio del tiempo, como muestra la Fig. 4.9 (a), donde la potencia y el ancho temporal se mantienen constantes (aproximadamente $5 \mathrm{~mW}$ y $3,5 \mathrm{~ns}$, respectivamente) en el tiempo de propagación. La Fig. 4.9 (b) se muestra el cambio en el espectro del pulso de entrada (ancho de banda aproximado $100 \mathrm{MHz}$ ) y el resultante que se ensancha (alrededor de 4,5 veces respecto al original) debido a la presencia del proceso no-lineal de la SPM en el cálculo de la salida del sistema.

La fase del pulso en el dominio del tiempo presenta una característica similar a la fase de un pulso con SPM, como se muestra en la Fig. 4.10 (a). Por otra parte, la transformada de Fourier del pulso tiene una fase con un chirp no-lineal inducido por la dispersión de segundo orden de las FOs, las FBGs y las no-linealidades del sistema, como puede observarse en la Fig.4.10 (b). De esta manera, podemos verificar la hipótesis definida en el desarrollo 
del modelo analítico, dado que la característica de la dispersión influye directamente en el cálculo de la no-linealidad.

El cálculo de la propagación de los pulsos se puede extender a 500 y hasta 1000 roundtrips, asegurando una solución estacionaria en el sentido que se ha discutido anteriormente. Para la mayoría de los casos, hemos supuesto que el pico de la potencia de los pulsos presentes en el medio activo es igual a $P_{i n}=\left|E_{i 1}\right|^{2} A_{\text {eff }} \approx 100 \mathrm{~mW}(20 \mathrm{dBm})$ y que el ancho temporal del pulso de entrada empleado para iniciar los cálculos igual a $T_{i n=} 2 \mathrm{~ns}$. El número de roundtrips necesario para encontrar una solución estable puede cambiar dependiendo de los parámetros de la Tabla 4.1 y las condiciones iniciales del pulso de entrada. Las características de este último, tales como su potencia, ancho temporal y forma, son elegidas convenientemente para asegurar una rápida convergencia. Sin embargo, si el pulso ingresado tiene atributos diferentes a los indicados, el sistema se encarga de ajustarlo de forma progresiva, alcanzando un resultado en un número de roundtrips mayor, a expensas de incrementar el tiempo de cálculo.

El valor inicial de la ganancia $g$ está definido por la Ec. 4.25, el cual depende del valor de $g_{0}$ mostrado en la Tabla 4.1 y de la intensidad del pulso dentro de la cavidad activa. Estos valores son compatibles con los valores de ganancia esperados y obtenidos de forma experimental ( $g L_{M}$ menor a $25 \mathrm{~dB}$ para amplificadores de $\mathrm{FO}$ dopada con $\mathrm{Er}^{3+}$ ), alcanzando una salida estable para un valor intensidad de pulso dentro de la cavidad activa de $I_{i n}=$ $P_{\text {in }} / A_{\text {eff }}=1,57 \times 10^{-3} \mathrm{~V}^{2} / \mathrm{\mu m}^{2}$. El valor de la potencia de saturación elegida para realizar los cálculos fue de $7 \mathrm{dBm}$ (dentro del rango de valores mencionados anteriormente).

\subsubsection{Establecimiento del régimen pulsado y $\mathrm{CW}$ mediante un proceso de ruido aleatorio}

Usualmente, una forma de obtener información de un sistema lineal o no-lineal es por medio del ruido, mediante procesos de identificación de sistemas desarrollados en la teoría de control moderno $[129,130]$. En general, los procesos de ruido son variados y dependen de la naturaleza física de los elementos que componen el sistema analizado. 
Nuestro modelo también permite estimar una solución para la condición similar al selfstarting del sistema mediante el uso de un pulso embebido en ruido. Esta condición inicial es análoga a la producida en un régimen de self-pulsing para la cual los sistemas láser emiten pulsos de potencia baja y de forma regular en el tiempo, como mencionamos anteriormente. Pequeñas señales de ruido generado por inestabilidades del medio de ganancia, relacionadas al régimen self-pulsing, podrían establecer un régimen mode locking si las longitudes de las cavidades son adecuadas. Una vez alcanzado este régimen, generalmente para una emisión láser de una mayor potencia, la influencia del régimen self-pulsing tiende a ser despreciable. En nuestro caso en particular aplicamos un pulso del tipo secante hiperbólica y de fase nula sumado con un proceso de ruido blanco gaussiano y aditivo a la entrada del modelo numérico, o sea como el campo $E_{i n}$ definido en la Fig. 4.4. El mismo se inyecta al sistema por medio de secuencias de largo finito (iguales al largo de la señal) y de forma tal que las diversas realizaciones de ruido no correlacionadas entre sí. El ruido generado y sumado al pulso mencionado se calcula mediante la envolvente compleja de un proceso pasabanda, empleando el modelo de ruido de fase y cuadratura como describe en su libro de comunicaciones Ziemer [102]. De todas formas, se debe notar que el proceso mencionado se limita en frecuencia mediante las características espectrales de las FBGs.

Empleando un total de 50 secuencias de ruido y un pulso de potencia $I_{\text {in }} \approx-50 \mathrm{dBm}$ con un ancho temporal de $T_{i n}=2$ ns se obtiene una baja relación señal a ruido igual a $\mathrm{SNR}_{\text {in }}=0,1 \mathrm{~dB}$ en la entrada del modelo. Dicho valor se define para el ancho de banda correspondiente al pulso de entrada, por lo que el pulso de entrada en el tiempo resulta inmerso totalmente en el ruido. En la Fig. 4.11 (a) se puede observar la evolución de la potencia del pulso ruidoso de salida del láser en función del número de viajes de ida y vuelta, donde el modo de operación pulsado presenta una mayor ganancia que el régimen de onda continua o CW. De esta manera, aún para pequeños valores de $\mathrm{SNR}_{\text {in }} \mathrm{y}$ para bajos niveles de ganancia $g_{0}$, el pulso resultante adquiere una $\mathrm{SNR}_{\text {out }}>\mathrm{SNR}_{\text {in }}$. En la Fig. 4.11 (b) se muestra el ancho temporal promedio del pulso ruidoso, el cual es cercano a 3,5 ns, con una desviación estándar moderada que está relacionada al bajo valor de $\mathrm{SNR}_{\text {in }}$. La $\mathrm{SNR}_{\text {out }}$ observada a 10 us (equivalentes a 500 viajes de ida y vuelta) fue de aproximadamente 

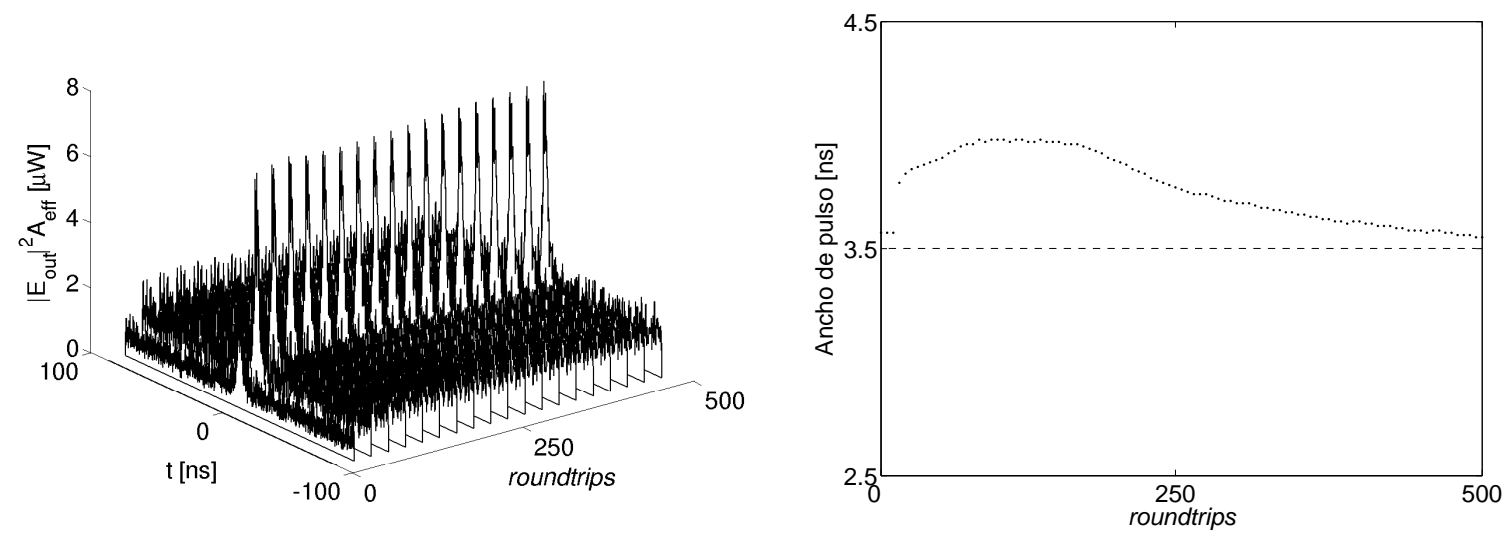

Figura 4.11: (a) Evolución temporal de la señal ruidosa de entrada en función del número de roundtrips. (b) Estimación del ancho promedio del pulso de salida.

10 dB. Además, se podría establecer un valor para la ganancia del régimen de operación pulsado como $P_{\text {out }} / P_{\text {in }} \approx 30 \mathrm{~dB}$, evaluando sólo los niveles de la señal óptica de interés. Por otra parte, el modo de CW crece desde el piso de ruido establecido mediante una potencia $\mathrm{N}_{\text {in }} \approx-50 \mathrm{dBm}$ hasta alcanzar un valor de potencia de salida próximo a los - $30 \mathrm{dBm}$, por lo cual se puede estimar una ganancia del régimen CW cuyo valor e próximo a $20 \mathrm{~dB}$. Sin embargo, cuando la potencia de ruido aleatorio es incrementada (por ejemplo, para un medio activo más ruidoso, o más intensamente excitado), el cálculo del término nolineal por medio de nuestro modelo resulta mucho más complejo, incrementando el tiempo de simulación. De todas formas, la ganancia del sistema puede ser regulada mediante la potencia de excitación del medio activo y el largo de la FO dopada con $\mathrm{Er}^{3+}$, dentro de los límites mencionados anteriormente.

Claramente, un proceso de ruido gaussiano y aditivo de potencia baja como la especificada permite determinar las principales características del sistema láser presentado, estableciendo ambos regímenes de funcionamiento (pulsado y CW). Estas propiedades también pudieron ser encontradas mediante el uso de señales determinísticas, donde se utilizaron pulsos para determinar el régimen pulsado, y en un análisis posterior, señales constantes en el tiempo para estimar alguna de las propiedades del régimen de operación $\mathrm{CW}$, obteniendo 


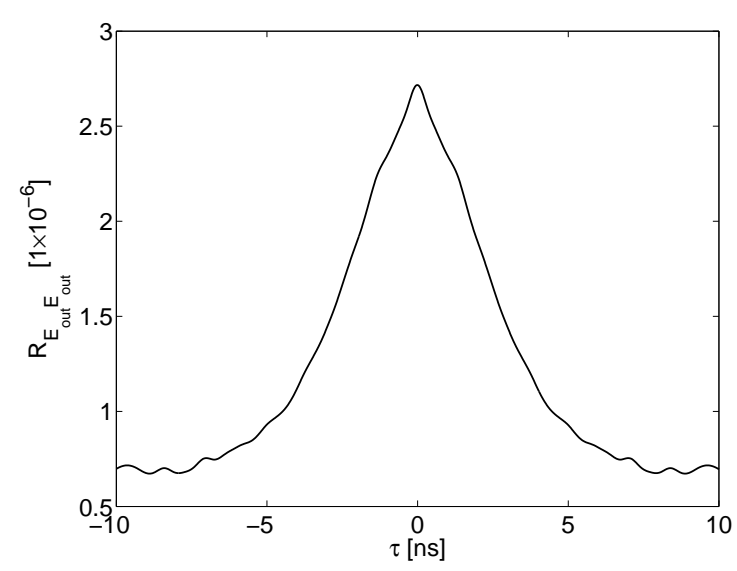

(a)

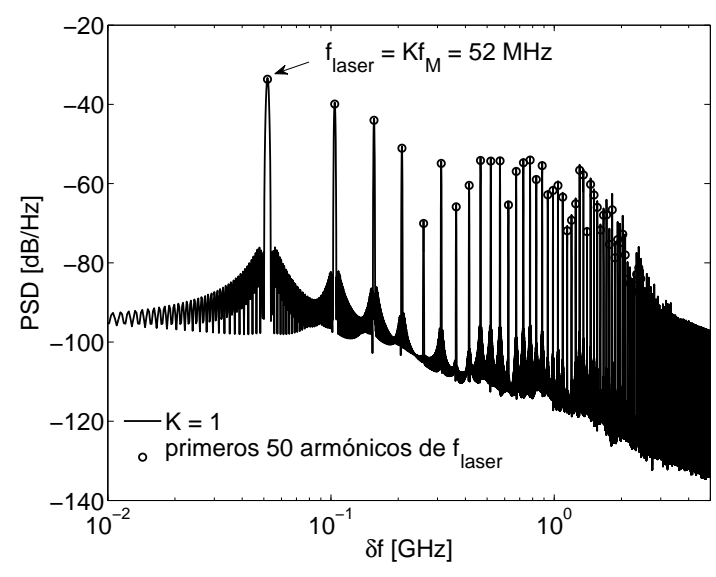

(b)

Figura 4.12: (a) Autocorrelación del proceso de salida en función de la diferencia de tiempos $\tau$. (b) Estimación de la PSD del proceso calculada mediante los últimos 5 us de simulación.

resultados similares a los mostrados en la Fig. 4.11. Por lo tanto, en la mayoría de los casos analizados a continuación hemos supuesto que los efectos del ruido son despreciables con el objeto de reducir el tiempo de cálculo. Este supuesto se basa en los valores adecuados de SNR encontrados de forma experimental, como se muestra en el Capítulo 5 bajo la condición de ajuste de la fase establecida para el régimen de operación mode locking.

En la Fig. 4.12 (a) se muestra la autocorrelación de la señal pulsada obtenida anteriormente, para la cual se ha desafectado el valor medio, correspondiente al régimen $\mathrm{CW}$. El ancho temporal de la autocorrelación es del orden de 6 ns. Esto indica que el ancho de pulso promedio, el cual se calcula tomando la mitad de dicho valor, sería algo menor al valor especificado de forma numérica para el caso de un pulso sin ruido. Por otra parte, se puede realizar una estimación de la densidad espectral de potencia (PSD) del proceso de salida empleando el periodograma de Welch [131]. Este algoritmo de procesamiento digital de señales se aplica dividiendo el vector de tiempo en un número finito de vectores más pequeños con igual número de puntos. Luego, se calcula la transformada de Fourier y se calcula el módulo al cuadrado, con el fin de promediar todos estos vectores y así obtener una estimación de la PSD. Para este caso en particular, se utilizan los pulsos 
calculados mediante el SSM durante un tiempo de simulación correspondiente a los últimos 250 roundtrips, o 5 us aproximadamente. El resultado de esta estimación puede ser vista en la Fig. 4.12 (b), donde el ancho de banda correspondiente al proceso de salida es del mismo orden que el ancho de banda del pulso sin ruido calculado anteriormente. Se puede observar también que las componentes espectrales están separadas en frecuencia cada $\Delta f_{M}=\Delta f_{A}=$ $52 \mathrm{MHz}$ (valor que arroja el cálculo de la Ec. 4.1 para una longitud $L_{M}=1,976 \mathrm{~m}$ ), para una relación entre longitudes de las cavidades principal y auxiliar unitaria.

\subsubsection{Acoplamiento de los campos provenientes de ambas cavi- dades del láser APM}

Extendiendo el modelo numérico explicitado anteriormente para longitudes de la cavidad auxiliar más pequeñas, o sea $L_{A}<L_{M}$, se pueden obtener mayores frecuencias de repetición de la emisión láser de salida, disminuyendo el ancho de los pulsos, como podremos observar en el Capítulo 5. El algoritmo aplicado en el cálculo de estos casos debe ser reacondicionado para seguir la lógica que se describe a continuación. Por ejemplo, cuando $K=$ 2, la envolvente del campo eléctrico $E_{i n}=E_{i 1}^{2}$ es transmitida dentro de la cavidad auxiliar y es reflejada por el espejo, siendo propagada hacia la $\mathrm{FBG}_{2}$ como $E_{i 2}^{2}$. En este mismo instante, el campo $E_{o 1}^{2}$ ha sido propagado una distancia $L_{M}$, obteniendo como resultado el campo eléctrico $E_{i 2}^{1}$ en la $\mathrm{FBG}_{1}$. El ciclo es completado cuando la envolvente del campo eléctrico reflejado $E_{o 2}^{1}$ es propagada en la cavidad principal hasta lograr que el campo $E_{i 1}^{2}$ actualizado, mientras que $E_{i 2}^{2}$ debe ser renovado para ser acoplado a $E_{i 1}^{2}$ mediante la Ec. 4.7, relacionada a la $\mathrm{FBG}_{2}$. En resumen, los campos de la cavidad auxiliar deben ser actualizados $K$ veces antes de ser acoplados con un nuevo campo $E_{i 1}^{2}$ (rama izquierda del diagrama de flujo especificado en la Fig. 4.8). Esta condición debe ser aplicada para cualquier relación de longitudes entera $K$, mientras que para relaciones racionales (por ejemplo, entre números coprimos) el análisis se vuelve más complejo, obligando a realizar cálculos intermedios e incrementando del uso de la memoria.

Aplicando el modelo numérico descrito anteriormente pero para valores de $K$ iguales a 1 , 


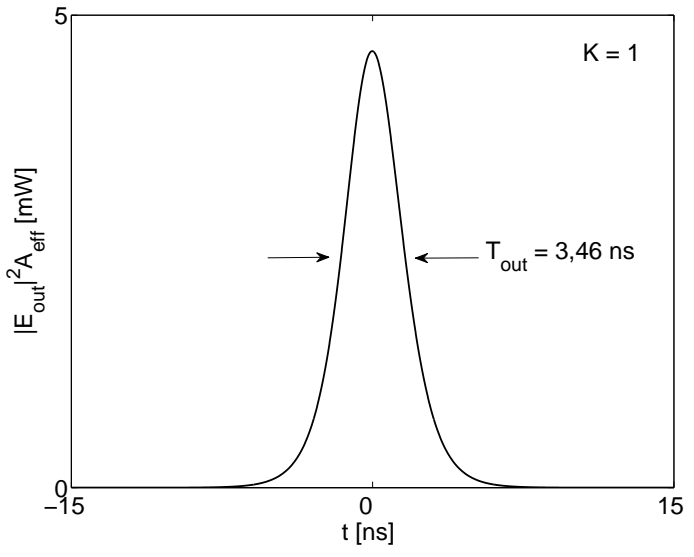

(a)

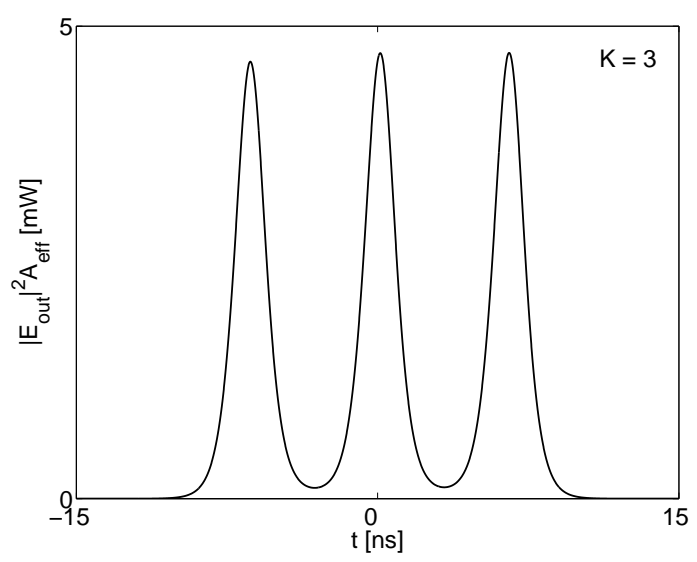

(c)

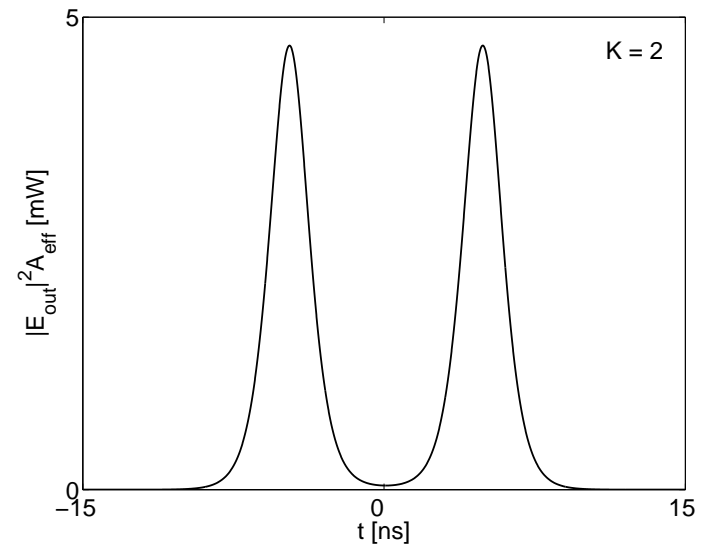

(b)

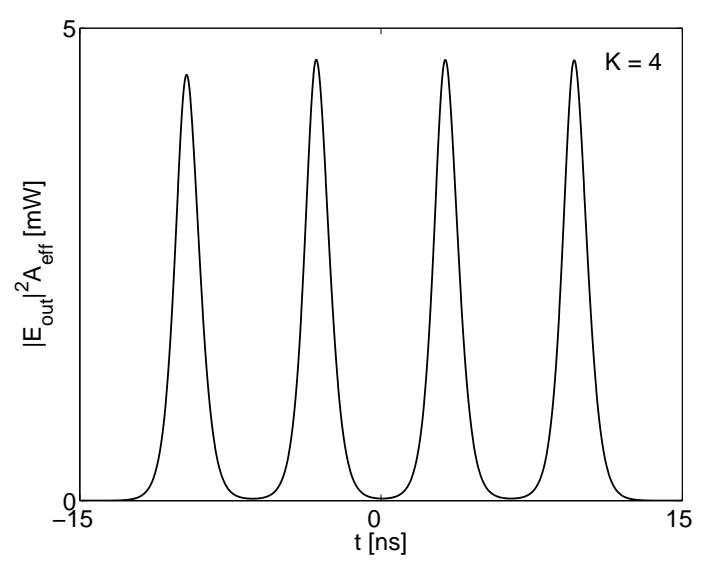

(d)

Figura 4.13: Simulación de la potencia del campo eléctrico de salida para diferentes valores de $K$. (a) $K=1$ ( $\left.T_{\text {out }}=3,46 \mathrm{~ns}\right)$. (b) $K=2$ ( $\left.T_{\text {out }}=2,26 \mathrm{~ns}\right)$. (c) $K=3$ ( $\left.T_{\text {out }}=1,74 \mathrm{~ns}\right)$. (d) $K=4\left(T_{\text {out }}=1,45 \mathrm{~ns}\right)$.

2, 3 y 4, y manteniendo constante la longitud de la cavidad principal del láser $L_{M}=1,976$ $\mathrm{m}$, se pueden obtener los resultados que se muestran a continuación. Para la mayoría de los casos, una solución estable fue encontrada para un número grande de roundtrips, donde la forma del campo eléctrico $E_{i 1}^{2}$ (potencia y ancho temporal) se mantienen aproximadamente constantes con el tiempo de propagación, como hemos observado anteriormente y en nuestros trabajos $[2,46]$. En la Fig. 4.13 se muestran los resultados numéricos para diferentes 


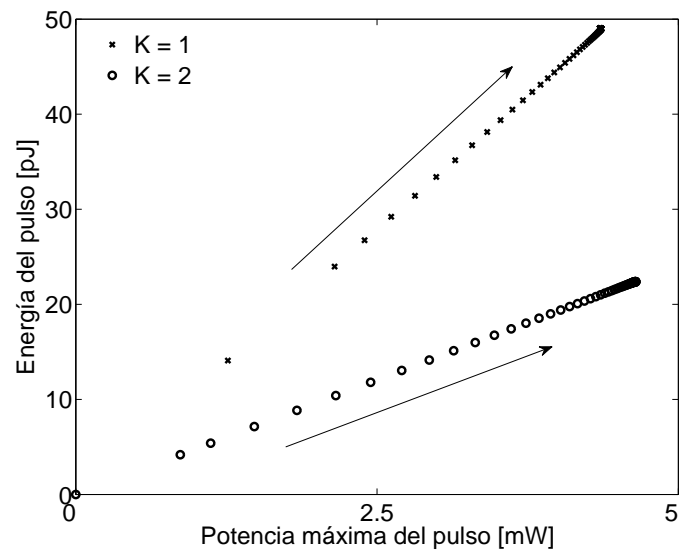

Figura 4.14: Evolución de la energía del pulso simulada en función de la potencia máxima y de la relación de longitudes $K=1$ y $K=2$.

relaciones entre longitudes: (a) $K=1$, (b) $K=2$, (c) $K=3$ and (d) $K=4$, donde $L_{M}=$ 1,976 m para todos los casos. En la Fig. 4.13(a) aparece un único pulso debido a que el tiempo de propagación para ambas cavidades es el mismo. Sin embargo, en las Figs. 4.13(b), 4.13 (c) y 4.13 (d) aparecen 2, 3 o 4 pulsos, respectivamente y en la misma ventana, dado que para un tiempo de roundtrips de la cavidad principal se están propagando $K$ pulsos en la cavidad auxiliar del láser. Como se describe anteriormente, esto depende de la frecuencia de batido resultante entre ambas cavidades, obteniendo una solución particular para cada caso. Los anchos de pulso obtenidos de forma numérica fueron: (a) 3,46 ns, (b) 2,25 ns, (c) 1,74 ns y (d) 1,45 ns.

En la Fig. 4.14 se muestra cómo se produce la convergencia de un pulso mediante el modelo numérico especificado hacia una solución estable para dos valores de relación de longitudes $K=1$ y $K=2$. Se observa que en los primeros instantes de tiempo, la energía del pulso se incrementa en pasos moderados, luego estos incrementos se vuelven más pequeños hasta encontrar una solución estable del sistema.

Una forma de determinar el número de componentes espectrales involucradas en el proceso de enganche de los modos es mediante el estudio de la PSD de la señal estimada numéricamente. En la Fig. 4.15 se puede observar el cambio del ancho de banda de la señal 


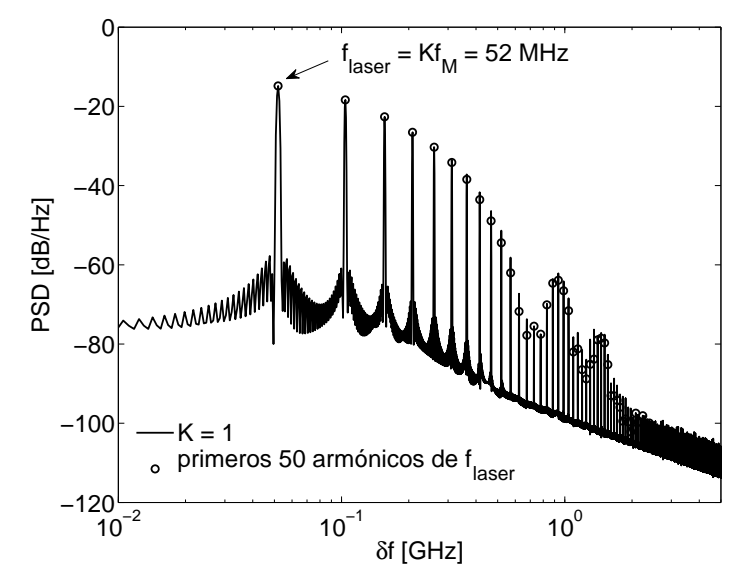

(a)

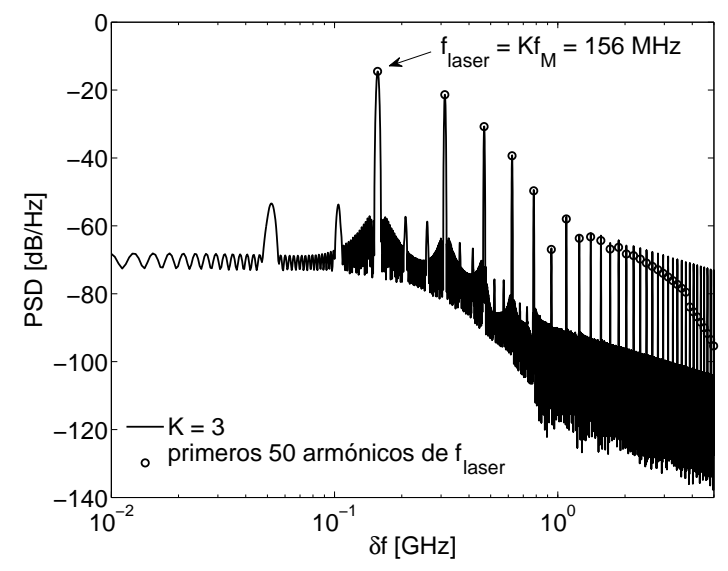

(c)

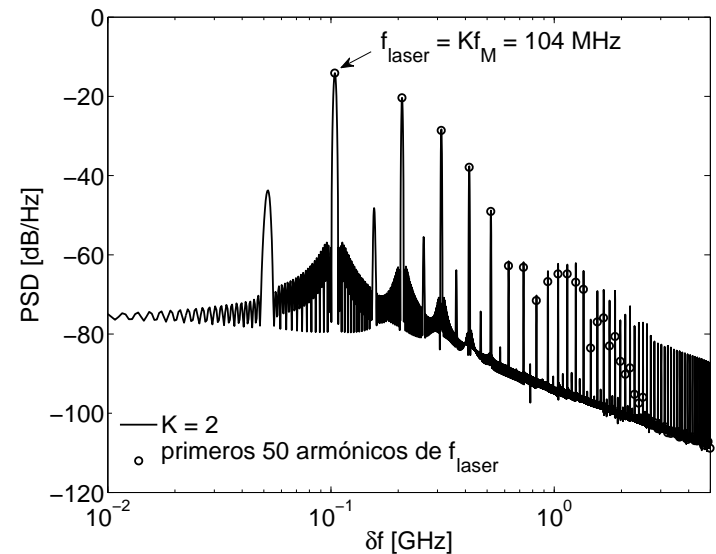

(b)

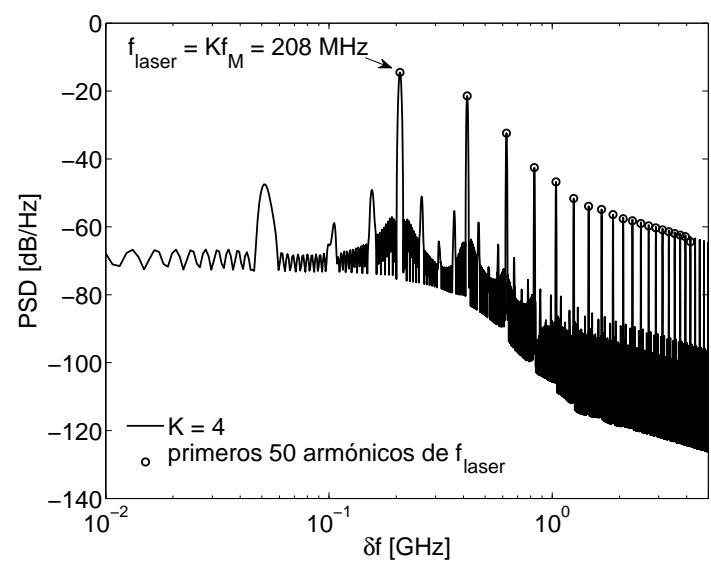

(d)

Figura 4.15: Estimación de la PSD de la señal de salida para distintos valores de $K$. El incremento en el ancho de banda de la señal de salida se debe principalmente a la disminución del ancho de pulso, cuando la longitud de la cavidad auxiliar $L_{A} \leq L_{M}$. Con "○" se muestran los primeros 50 armónicos correspondientes a cada frecuencia láser de salida. (a) $K=1$ ( $\left.T_{\text {out }}=3,46 \mathrm{~ns}\right)$. (b) $K=2$ ( $\left.T_{\text {out }}=2,26 \mathrm{~ns}\right)$. (c) $K=3$ ( $\left.T_{\text {out }}=1,74 \mathrm{~ns}\right)$. (d) $K=4\left(T_{\text {out }}=1,45 \mathrm{~ns}\right)$.

en función del valor establecido por la relación de longitudes de ambas cavidades, dada por la Ec.4.8. Estos resultados se encuentran empleando pulsos con ruido aditivo gaussiano, como los vistos en la sección anterior, pero para un valor de SNR elevado igual a $30 \mathrm{~dB}, \mathrm{y}$ 
con una potencia de señal dentro de la cavidad activa igual a 20 dBm. En la Fig. 4.15 (a), las componentes espectrales se encuentran separadas en frecuencia cada $\Delta f_{M}=52 \mathrm{MHz}$ $\left(L_{M}=1,976 \mathrm{~m}\right.$ y $\left.K=1\right)$, mientras que la distribución de amplitudes de cada componente queda definida por la característica espectral del sistema. Comparando dicho resultado con la 4.12 (b), se observa que el sistema admite que algunas componentes de alta frecuencia presenten una potencia mayor. Sin embargo, como el sistema presenta una característica en frecuencia del tipo pasabajos, el resultado obtenido en la Fig. 4.15 (a) puede ser considerado como una muy buena aproximación de lo que sucede en la realidad. Para las Figs. 4.15 (b), (c) y (d), correspondientes a las frecuencias de salida $f_{\text {laser }}=104 \mathrm{MHz}, 156 \mathrm{MHz}$ y $208 \mathrm{MHz}$, podemos observar que las componentes espectrales se encuentras espaciadas en valores múltiplos enteros de dichas frecuencias, mostrando una característica espectral similar a la mencionada para las altas frecuencias. De estos resultados también se puede notar que el ancho de banda de la señal generada se incrementa de forma proporcional al valor de $K$, mientras que el número de componentes espectrales definidas en un ancho de banda de $3 \mathrm{~dB}$ es pequeño (generalmente $<3$ ). Los anchos de banda de estas señales se encuentran entre 100-500 MHz aproximadamente, dependiendo del caso. En este sentido, en el Capítulo 5 realizaremos una medición de la PSD empleando un analizador del espectro óptico (OSA). En ese caso la resolución del instrumento sería una limitación en la determinación de las distintas componentes espectrales de la señal generada. Una solución a dicho inconveniente se presenta realizando una estimación de dichas componentes mediante la PSD de las señales fotodetectadas para distintas relaciones de longitud $K$. De esta forma, podremos observar una similitud muy grande entre los resultados numéricos y experimentales obtenidos.

\subsubsection{Efecto de la dispersión en la cavidad activa}

Empleando un modelo simplificado del sistema láser propuesto, el cual está basado en el subsistema formado por la cavidad principal, se puede estimar el efecto de la dispersión en la propagación de los pulsos dentro de la misma. El esquema de la Fig. 4.16 muestra una 


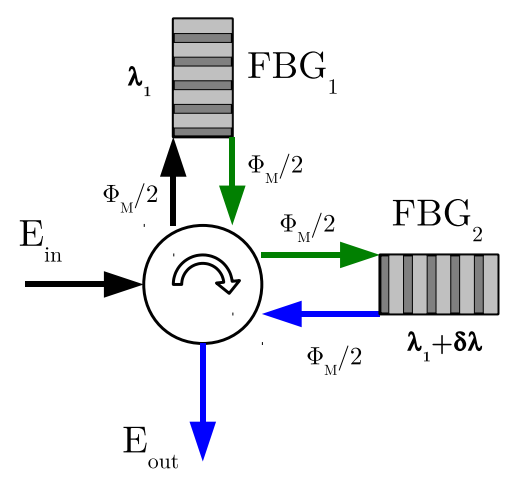

Figura 4.16: Esquema de la cavidad principal basada en la reflectividad de las FBGs.

configuración de dos FBGs y un circulador ideal conectado a las mismas mediante tramos de FO ideales (no introducen pérdidas, efectos de la dispersión y no-linealidades), donde se ha despreciado el efecto de la cavidad auxiliar, la cual será analizada posteriormente. Este esquema es fácilmente representado de forma analítica mediante la siguiente relación

$$
\frac{\tilde{E}_{\text {out }}(f)}{\tilde{E}_{\text {in }}(f)}=\rho_{F B G_{1}}(f) \rho_{F B G_{2}}(f) e^{-i 2 \Phi_{M}},
$$

suponiendo que $\tilde{E}_{\text {out }} / \tilde{E}_{\text {in }}$ expresa la respuesta de la cavidad principal mediante las características de reflexión de las FBGs y un factor de fase constante debido a la longitud del camino óptico recorrido, o sea $\Phi_{M}=\phi_{m}$. Sin embargo, debemos recordar que esta aproximación es sólo a los efectos de evaluar el comportamiento de la dispersión en dicha cavidad y supone que las FBGs se comportan como dispositivos lineales para el rango de longitudes de onda de trabajo. La dispersión de las FBGs, como ya hemos visto en el cálculo numérico, es un tema sensible en el ajuste de los términos de la fase, establecido por la Ec.4.32. Los valores de la dispersión estimados para valores pequeños de detuning, como se observa en la Fig.4.7 (b), pueden ser fácilmente alterados si se produce un desajuste entre las longitudes de onda de Bragg de las FBGs. Suponiendo que la propiedades de las FBGs son idénticas, se puede estimar el cambio en el perfil de la dispersión del subsistema analizado, como se observa en la Fig. 4.17 (a). Cuando la separación espectral entre las características de reflexión de las FBGs es nulo $\left(f_{B r a g g_{1}}=f_{\text {Bragg }_{2}}\right)$, la respuesta de dispersión del filtro es idéntica a la expresada en la Fig.4.7 (b), mientras que para otros valores $\left(f_{\text {Bragg }_{1}} \neq f_{\text {Bragg }_{2}}\right)$ 


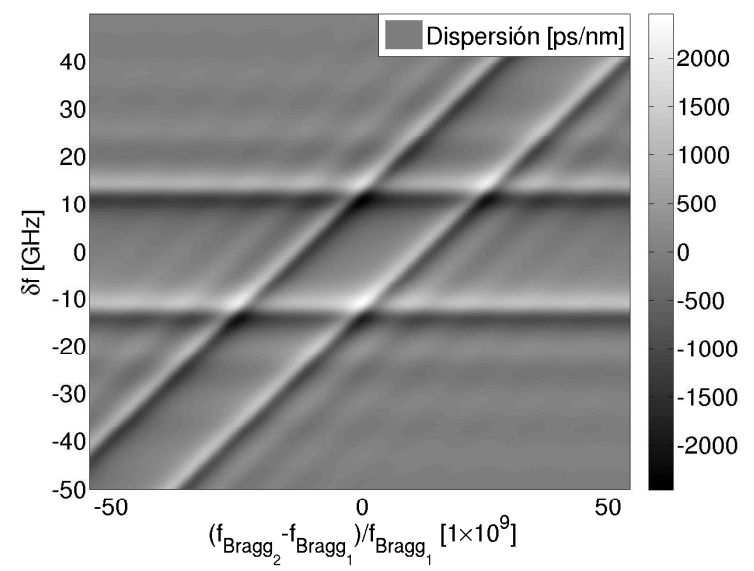

(a)

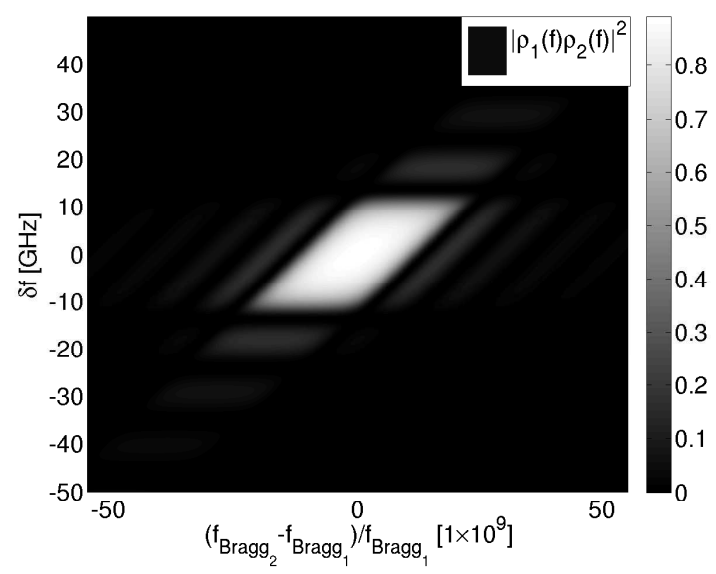

(b)

Figura 4.17: Características del filtro lineal formado por las FBGs que conforman la cavidad principal del láser APM para distintos valores de separación espectral entre las mismas, $\left(f_{\text {Bragg }_{2}}-f_{\text {Bragg }_{1}}\right) / f_{\text {Bragg }_{1}}$. (a) Dispersión de la cavidad, resultante del producto de los coeficientes de reflexión de las FBGs que la componen. (b) Producto de las reflectividades de ambas FBGs en función de la separación espectral relativa entre las mismas.

la dispersión se ve alterada fácilmente en módulo y signo. Por lo tanto, el subsistema puede generar pulsos más anchos o angostos (si no están limitados por transformada) en el dominio del tiempo dependiendo de la condición mencionada. Estos cambios en las longitudes de onda de las FBGs, en la práctica son producidos cambios en la temperatura ambiente, la cual puede influir de manera diferente en cada uno de los dispositivos que componen el sistema de realimentación del láser. Por otra parte, también se produce un cambio en la característica de reflectividad del subsistema, la cual puede ser expresada mediante el producto de las reflectividades individuales de las FBGs, como se observa en la Fig. 4.17 (b). Este efecto se traduce en un cambio de la condición de acoplamiento, la cual podrá ser analizada en la próxima sección mediante un operador de la reflexión de la cavidad auxiliar.

Luego, si introducimos la dispersión de la FO dopada con $\mathrm{Er}^{3+}$, o sea $\Phi_{M}=\phi_{m}+\beta_{2 M} L \omega^{2} / 2$, no se observan cambios significativos en la característica de la dispersión mostrada en la Fig. 4.17 (a). Esto se debe principalmente a que el valor de la dispersión cromática de la FO 
dopada es pequeño comparado el de las FBGs (para $\lambda_{0}=1553,55 \mathrm{~nm}$ ), para las potencias de bombeo consideradas (menores a $325 \mathrm{~mW}$ ), como mostraremos en el Capítulo 5. Sin embargo, no se puede despreciar dicho aporte ya que la dispersión cromática en dichas FOs cambia significativamente en módulo y signo, entre dos y tres órdenes de magnitud, dependiendo de la longitud de onda de la emisión láser generada, como ya hemos mencionado en el Capítulo 2.

\subsubsection{Coeficiente de reflexión no-lineal de la cavidad auxiliar}

El comportamiento del sistema láser esquematizado en la Fig. 4.1 puede ser estudiado mediante el empleo de otro subsistema compuesto por los dispositivos fotónicos que conforman la cavidad auxiliar. El análisis de dicho subsistema debe realizarse bajo la condición de acoplamiento de los campos eléctricos que provienen de ambas cavidades, debido a que los resultados dependen principalmente de las no-linealidades introducidas por las mismas. Observando la reflexión generada por este subsistema, definida mediante los campos eléctricos de entrada/salida de la $\mathrm{FBG}_{2}$ (cavidad activa), se puede escribir la siguiente relación

$$
\Gamma_{a}(t)=\frac{E_{o 1}^{2}(t)}{E_{i 1}^{2}(t)},
$$

donde $\Gamma_{a}(t)$ es un coeficiente de reflexión del subsistema mencionado. En condiciones generales, como la potencia de los pulsos dentro de dicha cavidad es baja (menores a 10 mW para FBGs de $93 \%$ ), para los casos analizados, este subsistema se comporta como un filtro cuasi-lineal (para el rango de potencias de pulso y reflectividades de las FBGs evaluadas). Sin embargo, debemos recordar que pequeños cambios en el módulo y la fase de la Ec. 4.35, introducidos principalmente por alteraciones en los dispositivos, pueden alterar las características tiempo-frecuencia del sistema. En la Fig. 4.18 (a) se muestra el módulo del coeficiente de reflexión para una potencia de entrada igual a $P_{\text {in }}=\left|E_{\text {in }}\right|^{2} A_{\text {eff }}=100$ $\mathrm{mW}$ (valor máximo en la cavidad principal), empleando un ancho de pulso $T_{i n}=2 \mathrm{~ns}$. Se puede observar que el valor de la reflectividad del filtro es alto, para FBGs con valores de 


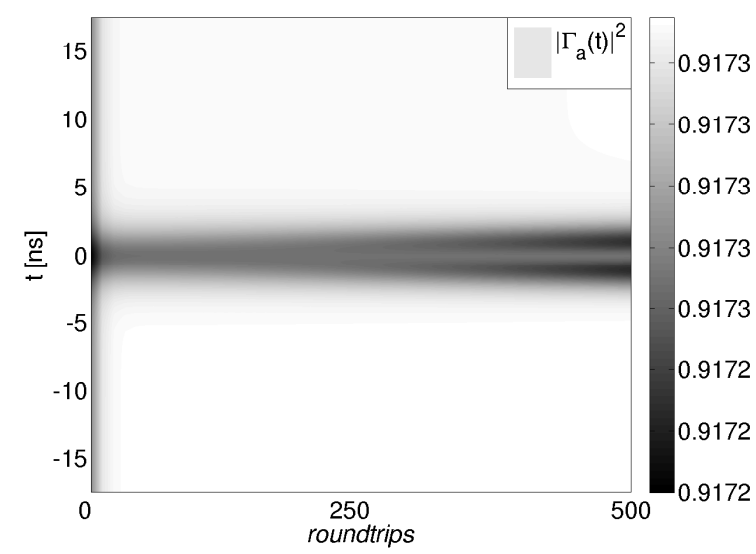

(a)

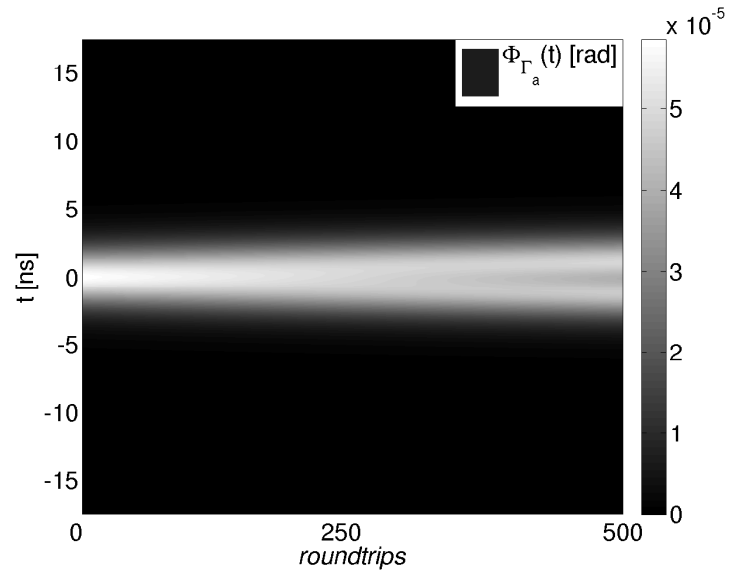

(b)

Figura 4.18: Característica temporal del coeficiente de reflexión $\Gamma_{a}(t)$, correspondiente al subsistema formado por la cavidad auxiliar. (a) Reflectividad del mismo en función del número de roundtrips. (b) Respuesta de fase del mismo.

reflectividad de $93 \%$, mientras que las fluctuaciones pequeñas de $\left|\Gamma_{a}\right|^{2}$ se producen para valores de tiempo $t \approx 0$. Estas fluctuaciones están asociadas al incremento de la distorsión en las colas del pulso propagado, debida a la fase no-lineal. En la Figs. 4.18 (b) se observa la fase del coeficiente de reflexión, la cual presenta cambios pequeños del orden de la centésima de miliradian (dentro de la cota de error establecida para el cálculo de la propagación mediante el SSM).

Por otra parte, cuando el valor de la reflectividad de las FBGs que componen el láser disminuye, el subsistema mencionado se comporta de manera ligeramente diferente. En las Figs. 4.19 (a) y (b) se muestra la reflectividad y la fase del coeficiente de reflexión del subsistema para un par de FBGs de $75 \%$ de reflectividad. Mediante la colección de distintos resultados se pudo observar que el valor de la reflectividad media del filtro disminuye a $\left|\Gamma_{a}\right|^{2} \approx 0,65$, presentando nuevamente pequeñas variaciones para $t \approx 0$. El comportamiento del filtro es levemente diferente al observado en la Fig. 4.18, debido a que la respuesta es prácticamente proporcional al perfil del pulso obtenido a la salida del láser. Esto se debe a la disminución parcial del proceso no-lineal del sistema. La misma conclusión resulta de 


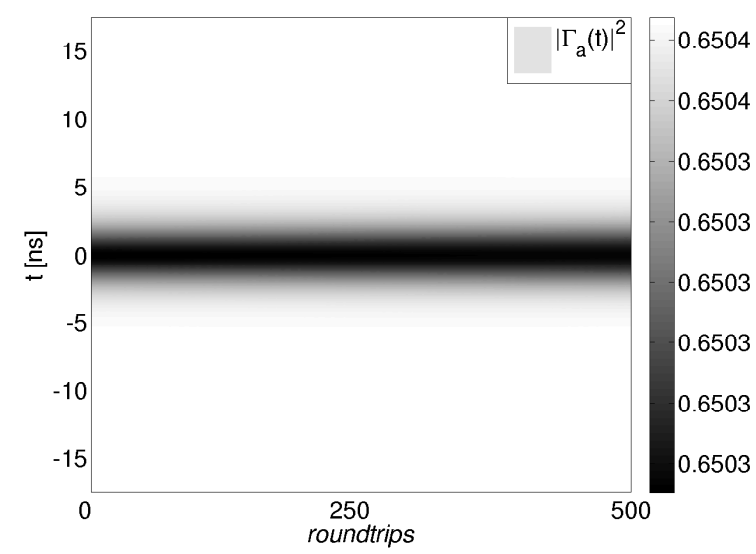

(a)

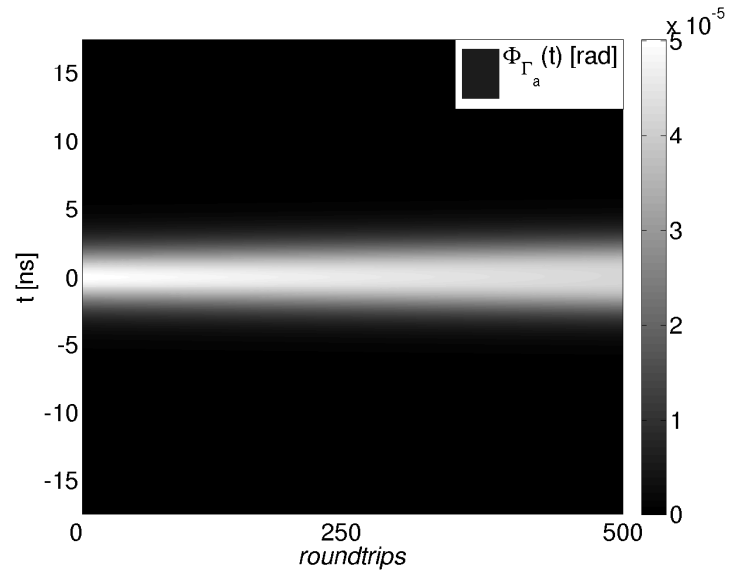

(b)

Figura 4.19: Característica temporal del coeficiente de reflexión $\Gamma_{a}(t)$. La reflectividad de las FBGs es del 75\%. (a) Módulo al cuadrado del coeficiente de reflexión del subsistema. (b) Respuesta de fase del mismo.

observar las respuestas de la fase del coeficiente de reflexión del subsistema en las Figs. 4.18 (b) y 4.19 (b). Suponiendo que los valores de las reflectividades de las FBGs son orden de $70 \%$ o menores se produce un incremento de la intensidad del proceso de acoplamiento de los campos eléctricos provenientes de ambas cavidades, aunque la potencia de dichos campos disminuye. De todas formas modificaciones en las características espectrales de los dispositivos empleados pueden introducir inestabilidades indeseadas en el sistema, como veremos a continuación.

\section{Cambios en la reflectividad del semiespejo de la cavidad auxiliar del làser}

En general, para los parámetros del sistema definidos en la Tabla 4.1, los cambios de la característica definida por la Ec. 4.35 no son muy significativos. Este comportamiento se debe a que el valor del coeficiente de reflexión del semiespejo, formado en el extremo de la FO estándar de la cavidad auxiliar pequeño, del orden de $4 \%$.

Un incremento en la reflectividad del semiespejo induce un aumento de la potencia de los campos eléctricos realimentados, incrementando la influencia del proceso no-lineal de la 


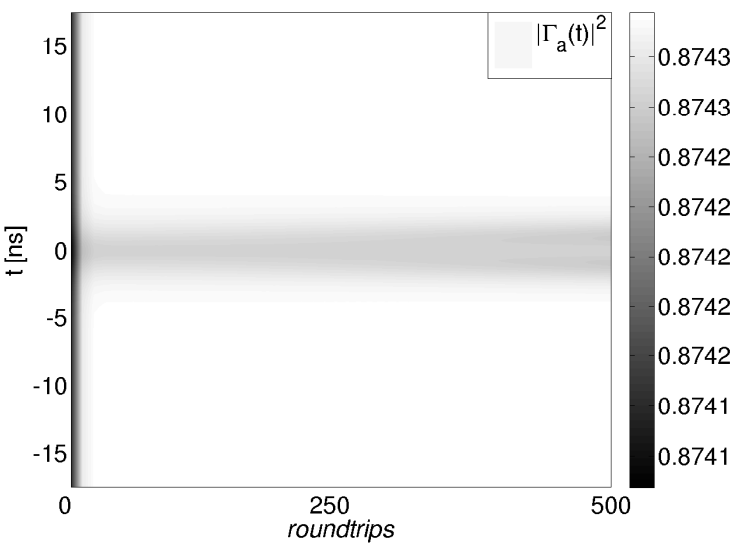

(a)

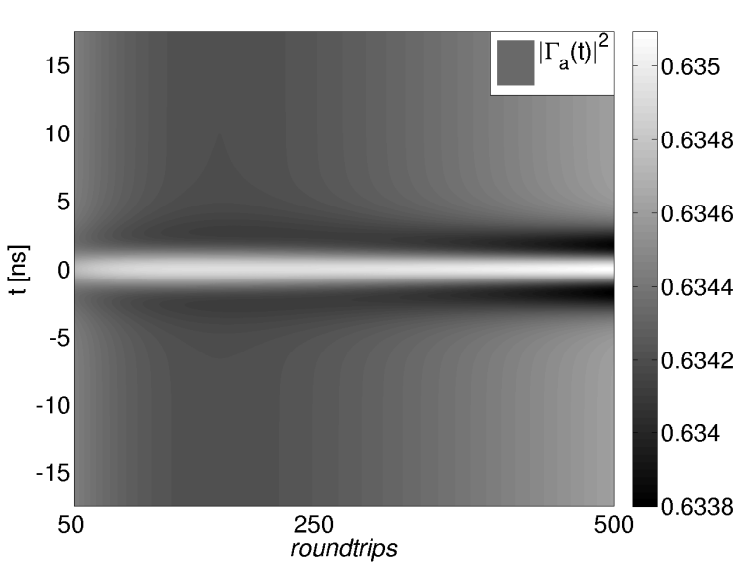

(c)

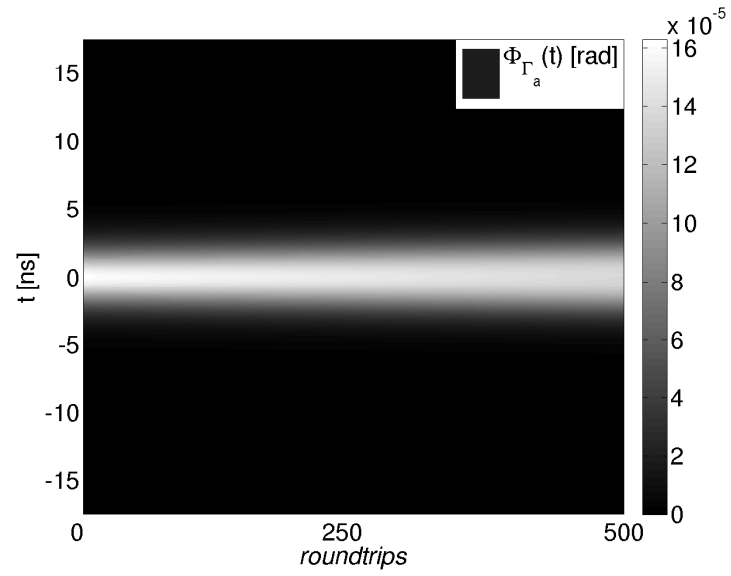

(b)

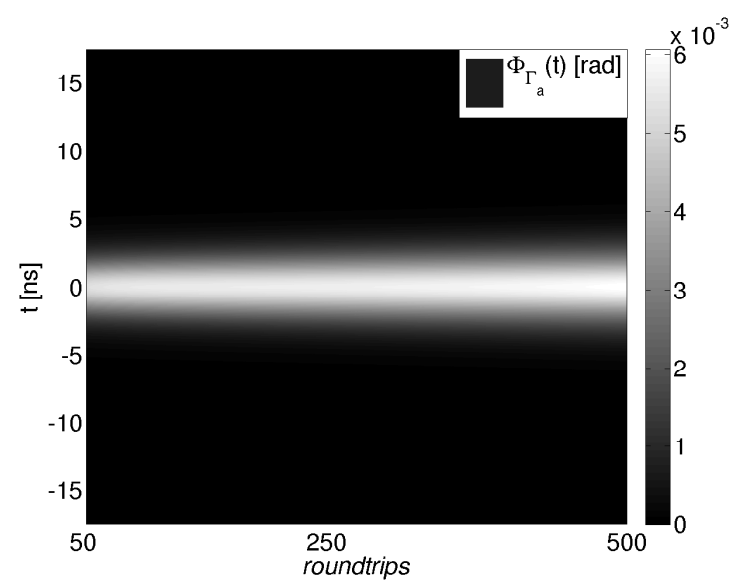

(d)

Figura 4.20: (a) y (c) Reflectividad de la cavidad auxiliar para $\left|\rho_{\text {mirror }}\right|^{2}=0,16$ y 0,6 , respectivamente. (b) y (d) Fase del coeficiente de reflexión de la cavidad auxiliar para $\left|\rho_{\text {mirror }}\right|^{2}=0,16$ y 0,60 , respectivamente.

SPM en la cavidad auxiliar del láser (aún para FBGs con valores de reflectividad elevadas). En la Figs. 4.20 (a) y (c) se muestra una disminución de $\left|\Gamma_{a}\right|^{2} \approx 0,87$ y 0,63 para $\left|\rho_{\text {mirror }}\right|^{2}=$ 0,16 y 0,6, respectivamente, en función del número de roundtrips. Las Figs. 4.20 (b) y (d) expresan el incremento de los valores de la fase de $1,5 \times 10^{-6} \mathrm{rad}$ a $6 \times 10^{-3} \mathrm{rad}$ aproximadamente, en forma proporcional al aumento de $\left|\rho_{\text {mirror }}\right|^{2}=0,16$ y 0,6 , respectivamente. Dicho incremento es de alrededor de 100 veces mayor a la cota de error máxima establecida para 
el cálculo de la propagación mediante el SSM. Un aumento significativo del valor de la fase es importante para establecer un criterio de estabilidad. Como podremos observar en la próxima sección, un valor de reflectividad del semiespejo $\left|\rho_{\text {mirror }}\right|^{2}>0,60$ correspondiente a un valor de fase mayor a $6 \times 10^{-3}$ rad puede alterar la condición de ajuste de la fase establecida para el régimen de operación mode locking.

\subsubsection{Inestabilidades del sistema}

Un análisis de estabilidad en láseres APM es importante para definir las condiciones donde se establece el regimen mode locking, como han estudiado de forma minuciosa en sus trabajos Haus [31,32] y Nielsen [23], entre otros. Las condiciones de estabilidad del sistema generalmente se basan en un análisis de la dispersión, del chirp inducido por la no-linealidad, de las distorsiones introducidas por el sistema de realimentación de la emisión generada y los distintos parámetros del sistema propuesto. Particularmente, en [31] se discuten los cambios en el ancho temporal y espectral de los pulsos en función de la dispersión total de un sistema con dos cavidades acopladas mediante el uso de un semiespejo. En este sentido, podemos realizar dos análisis sencillos mediante el modelo numérico propuesto: i) modificando la reflectividad de las FBGs (conservando el ancho de banda), como hemos observado en la sección anterior; ii) incrementando la potencia de los campos eléctricos propagados mediante un aumento de $\left|\rho_{\text {mirror }}\right|^{2}$ (reinyectando una señal de mayor potencia al sistema).

Suponiendo que un incremento sustancial de los valores de la fase puede generar algún tipo de inestabilidad de la salida, al menos en el sentido definido en la Sección 4.3 (salida estacionaria de potencia uniforme), es posible realizar un análisis preliminar aumentando la potencia de las señales (mediante un aumento de $\left|\rho_{\text {mirror }}\right|^{2}$ ). Volviendo al resultado expresado en la Fig. 4.20, habíamos mencionamos que la reflectividad promedio de la cavidad auxiliar disminuía de forma inversamente proporcional al aumento de $\left|\rho_{\text {mirror }}\right|^{2}$, mientras que para valores mayores al $60 \%$ se generan respuestas oscilatorias en función del tiempo de propagación, por lo que la salida es modulada en potencia y fase. Este 


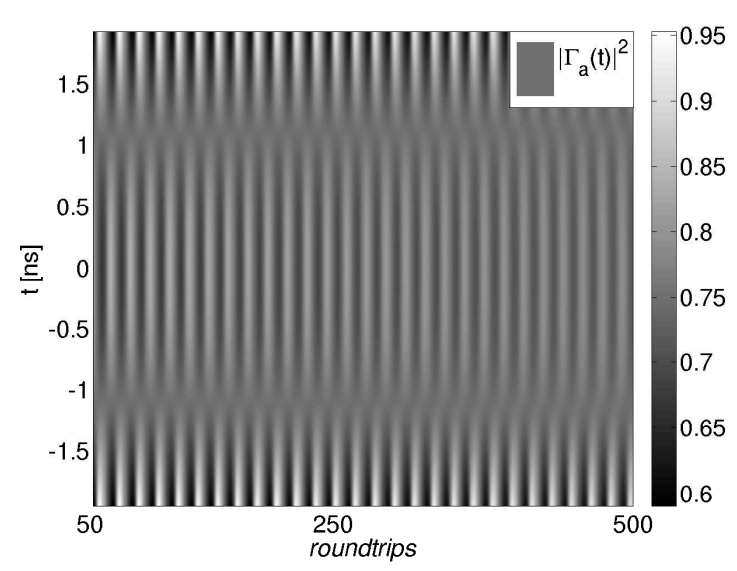

(a)

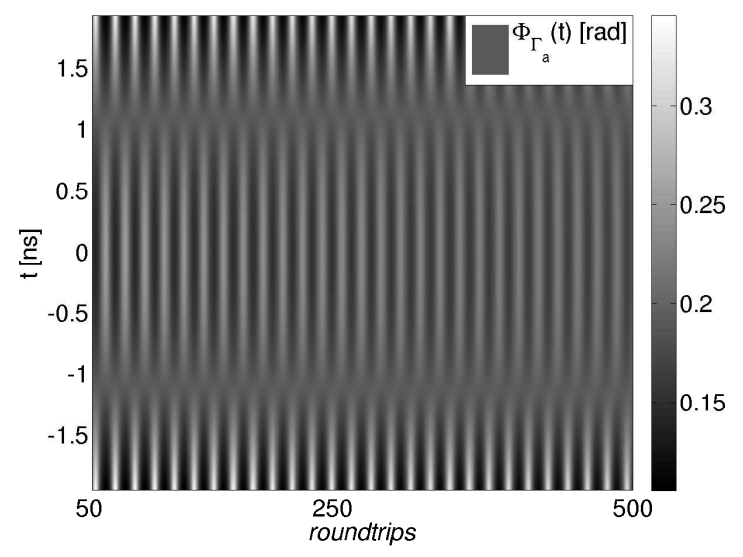

(b)

Figura 4.21: Modulación del coeficiente de reflexión definido para la cavidad auxiliar del sistema en función del tiempo de propagación para $\left|\rho_{\text {mirror }}\right|^{2}=0,75$. (a) Reflectividad. (b) Fase del coeficiente de reflexión de la cavidad auxiliar.

caso particular se puede observar en la Fig. 4.21 para un valor $\left|\rho_{\text {mirror }}\right|^{2}=0,75$, donde dicha modulación de la reflectividad del subsistema compuesto por la cavidad auxiliar (acoplada a la cavidad principal) es generalmente indeseada. Esta condición se expresa como inestable en sistemas láser que funcionan bajo el régimen additive-pulse mode locking ya que la potencia y el ancho de pulso de la emisión de salida resultante se encuentran modulados en el tiempo. Esta inestabilidad es producida por un desajuste de la fase debida a un incremento en la no-linealidad del sistema, y por lo tanto, se altera la condición de ajuste de la fase dada por la Ec. 4.32. De esta manera se puede establecer un límite o cota del valor de reflectividad del semiespejo, como ya hemos establecido anteriormente $\left(\left|\rho_{\text {mirror }}\right|^{2}<0,6\right.$, o bien, $\left.\Delta \Phi<6 \times 10^{-3} \mathrm{rad}\right)$, para la cual se puede asegurar que el sistema presenta variaciones suaves de la envolvente del campo eléctrico. De todas formas, debemos recordar que dicho límite puede cambiar en función de los parámetros establecidos en la Tabla 4.1.

Para el caso mostrado en la Fig. 4.21 podemos extender el análisis a un tiempo de propagación mayor para asegurar la solución. De esta manera se puede comprobar que la inestabilidad persiste en el tiempo, donde la potencia máxima y el ancho del pulso son 


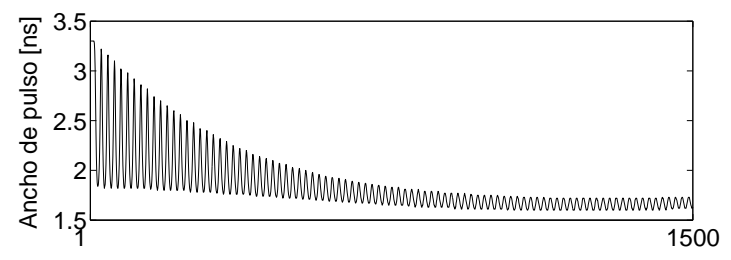

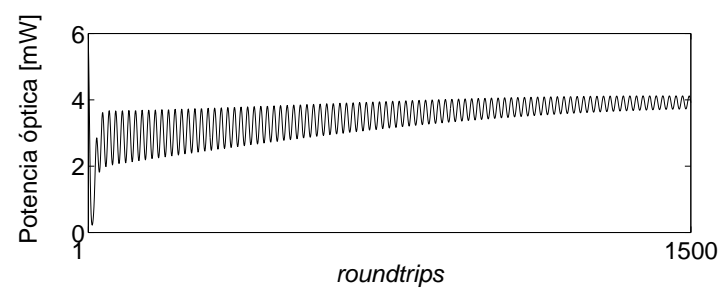

(a)

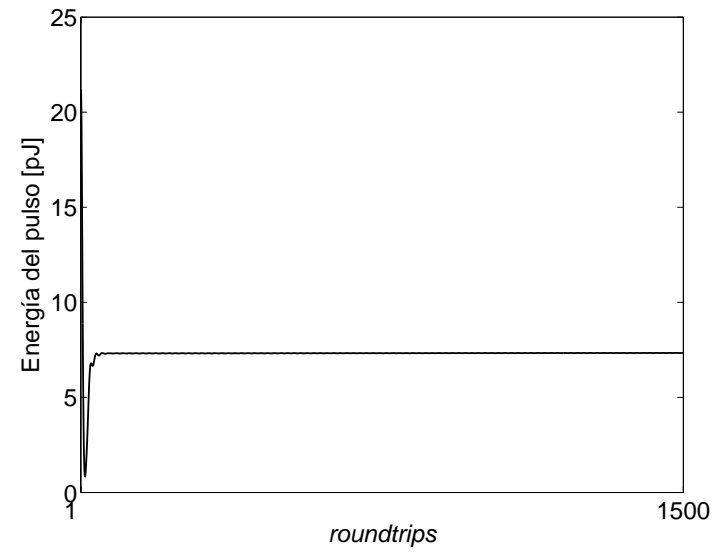

(b)

Figura 4.22: Inestabilidad producida por el aumento en la reflexión del semiespejo formado en el extremo de la SMF28 de la cavidad auxiliar. (a) Ancho temporal del pulso y potencia máxima del pulso en función del tiempo de propagación para $\left|\rho_{\text {mirror }}\right|^{2}=0,75$. (b) Evolución de la energía del pulso en función del tiempo de propagación, la cual permanece constante luego de un breve transitorio.

modulados en función del tiempo de propagación, como se observa en la Fig.4.22 (a). Una manera de verificar este resultado es calcular la energía del pulso en función del número de roundtrips, la cual resulta constante, como se puede observar en la Fig.4.22 (b). Además, de estos resultados se observa que la inestabilidad evoluciona con el tiempo de propagación hacia valores más pequeños, aunque aún inestables. Esto último puede ser visualizado también mediante un plano que expresa la potencia máxima del pulso en función de la energía, como se muestra en la Fig. 4.23 (a), la cual incluye el transitorio inicial. En la Fig. 4.23 (b) se muestra un aumento de la Fig. 4.23 (a) en la zona de energía próxima a 7,32 pJ, donde la inestabilidad se mantiene aproximadamente acotada para un tiempo de propagación largo (aproximadamente 20 ps). Este resultado es comparable a un "ciclo limite" (bucle prácticamente cerrado donde se establecen las soluciones del sistema) obtenido de un plano de fase de un oscilador basado en la generación de armónicas mediante un proceso no-lineal, como los estudiados en dinámica de sistemas no-lineales [130,132]. Respecto a la 


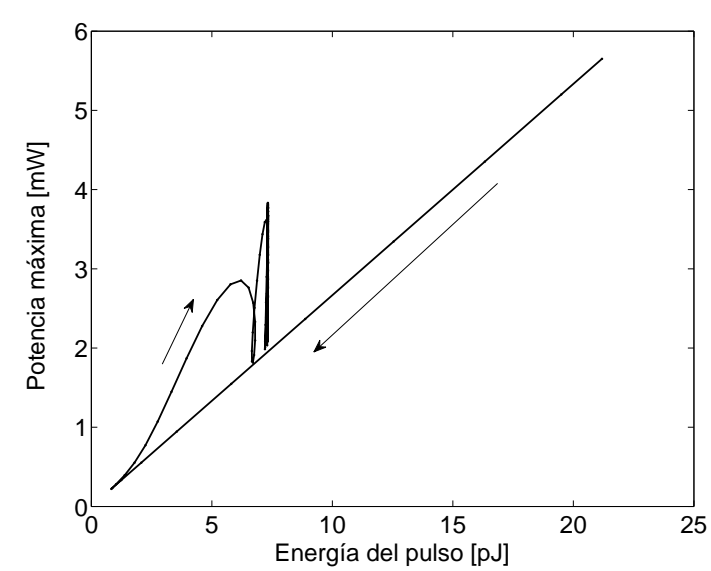

(a)

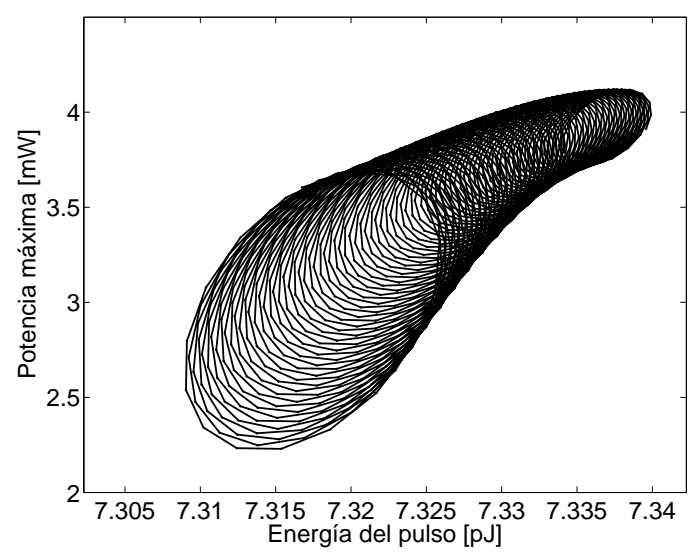

(b)

Figura 4.23: Evolución de la solución del sistema para $\left|\rho_{\text {mirror }}\right|^{2}=0,75$. La inestabilidad manifiesta en los cambios del ancho de pulso y la potencia de los mismos queda expresada en el plano potencia en función de la energía. (a) Evolución de la solución desde el inicio de la propagación. (b) Oscilación generada luego del transitorio inicial (aumento de la zona más densa de (a)).

verificación experimental, de forma preliminar hemos encontrado algunos resultados que comprueban la existencia de dichas inestabilidades, empleando un colimador de FO para 1,55 $\mathrm{mm}$ (en reemplazo del semiespejo) y un espejo de alta reflectividad. Sin embargo, este estudio se propone como una de las líneas de investigación futuras.

En resumen, empleando el modelo numérico propuesto, se puede encontrar una relación entre el valor de la reflectividad del semiespejo y la frecuencia de la inestabilidad. En la Fig. 4.24 se presenta dicha tendencia para diferentes valores de $\left|\rho_{\text {mirror }}\right|^{2}>60 \%$ y se observa que para valores de reflectividad del semiespejo mayores al $87 \%$ no se puede asegurar una convergencia para una cota de error de la fase de $1 \times 10^{-4}$ rad, fijada para el cálculo de la propagación de los campos eléctricos con el SSM. Esta característica, en principio, no se corresponde con ningún otro parámetro físico del sistema láser propuesto y sólo se debe a la fuerte interacción de los procesos lineales y no-lineales producidos en ambas cavidades del láser. La comprobación experimental de dicha característica se deja para trabajos futuros. 


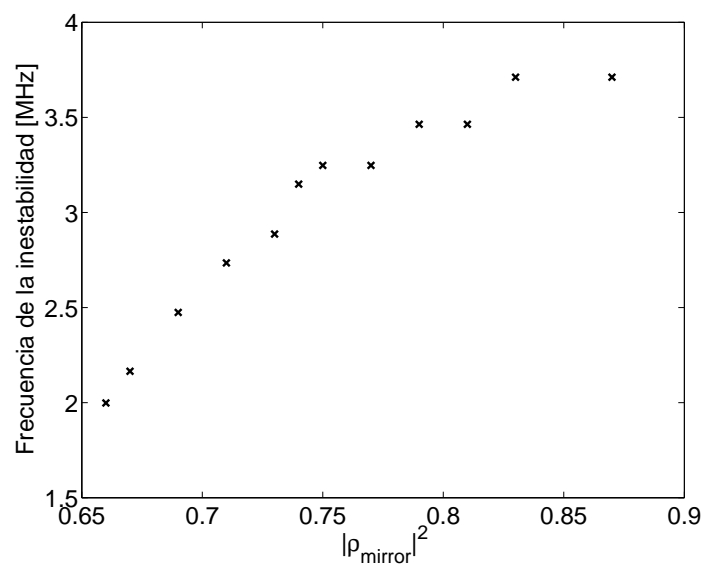

Figura 4.24: Frecuencia de la inestabilidad en función de la reflectividad del semiespejo. Para valores de $\left|\rho_{\text {mirror }}\right|^{2}$ levemente mayores al $60 \%$ las inestabilidades son oscilatorias, aunque generalmente se desarrollan de forma monótona, algunas hasta alcanzar otra condición estable. Para valores mayores, las inestabilidades de mantienen en el tiempo como se puede observar de la Fig. 4.23. Valores de $\left|\rho_{\text {mirror }}\right|^{2}>0,9$ complican el cálculo de la propagación mediante el SSM, debido al incremento del proceso no-lineal de la SPM.

\subsection{Discusión de los resultados numéricos}

El modelo numérico propuesto, basado en el SSM y el acoplamiento de los campos mediante la matriz de scattering propuesta, permite calcular la salida de un láser de FO que trabaja en régimen APM. La ventaja de estos métodos, aplicados en nuestro modelo, es una gran flexibilidad y fácil implementación de los algoritmos para todos los casos analizados. Otras configuraciones láser, por ejemplo, con cavidades en forma de anillo, pueden ser analizadas fácilmente alterando levemente el algoritmo empleado. El modelo numérico presentado admite las características espectrales completas de las FBGs, calculadas mediante el TMM, y el costo computacional es reducido en la mayoría de los casos, permitiendo obtener resultados precisos en tiempos cortos. Por otra parte, el modelo numérico implementado permite estimar la salida del láser para relaciones entre las longitudes de las cavidades no enteras, como la observada en [1], aunque dicho algoritmo debe ser modificado para cada caso de análisis en particular. Con respecto al potencial del SSM con paso variable, se espera una 
mejora en el modelado de sistemas láser de pulsos ultracortos que funcionan bajo el régimen APM, incluyendo procesos tan complejos como el FWM y la XPM. La incorporación de este tipo de procesos en el calculo de la propagación de los campos eléctricos en ambas cavidades implica que se deba tener en cuenta la variación de los distintos parámetros con la longitud de onda. En este sentido se debe calcular un número de ecuaciones acopladas no-lineales igual al número de componentes espectrales evaluadas, como menciona Agrawal en su libro de telecomunicaciones [5], incrementando el tiempo de cómputo y la complejidad de los algoritmos.

La resultados numéricos obtenidos son principalmente dependientes de la condición de ajuste de la fase, de las longitudes de ambas cavidades y principalmente de los parámetros físicos de las FOs empleadas y las FBGs. Además, se encontró que el acoplamiento de los campos eléctricos propagados en ambas cavidades se vuelve más intenso cuando el valor de reflectividad $\left|\rho_{3}\right|^{2}>>0,04$, para cuyos valores se produce una fuerte realimentación de la emisión generada en la cavidad principal del láser, llegando a perder la estabilidad de la salida del sistema, como hemos podido observar en las últimas secciones. Nuestro modelo también admite una solución estable para un valor de reflectividad nulo $\left|\rho_{3}\right|^{2}=$ 0, el cual corresponde al caso en el cual la cavidad auxiliar se desacopla de la cavidad activa. Este comportamiento será discutido en el Capítulo 5. La influencia de cada uno de los dispositivos constitutivos del sistema láser en la emisión del mismo, es de interés en el diseño y optimización para su aplicación en sistemas de comunicación y metrología óptica. 


\section{Capítulo 5}

\section{Implementación del láser APM de cavidades acopladas}

En este capítulo se muestran los resultados experimentales obtenidos mediante la implementación del sistema láser de cavidades acopladas presentado en el Capítulo 4. En la Sección 5.1 se describen los distintos dispositivos que fueron empleados en la implementación del láser y se realiza una caracterización espectral de las FBGs. En la Sección 5.2.1 se muestran las mediciones de la salida del láser para relaciones enteras entre ambas longitudes de cavidad ( $K=L_{M} / L_{A}$ enteros, con $L_{M}=1,976 \mathrm{~m}$ ). Se realiza una caracterización de la potencia de salida obtenida en función de la potencia de excitación aplicada verificando un umbral pequeño para la obtención de la emisión láser. Además, se implementan dos láseres nuevos cuyas longitudes de cavidad principal son $2 L_{M}$ y $3 L_{M}$, respectivamente, con el fin de evaluar el peso de algunos procesos no-lineales como XPM y FWM (los cuales son comunes en régimen APM). En general, estos procesos producen distorsiones en las características tiempo-frecuencia de las señales generadas (los pulsos cambian sus forma en el tiempo y surgen nuevas componentes espectrales). Despreciando estos efectos, como veremos a continuación, podremos resumir todos los resultados obtenidos en una característica que describe el ancho temporal de los pulsos en función de la frecuencia de la emisión de salida del sistema láser. Además, se lleva a cabo una discusión contrastando las mediciones 
obtenidas en [1] y las realizadas en nuestro trabajo [2]. En la Sección 5.2.2 se presentan algunas mediciones realizadas para relaciones de longitudes no-enteras $(K=M / N$ racionales, con $M$ y $N$ números primos) las cuales son de interés en la descripción del funcionamiento del régimen APM (influencia de las longitudes de FO de ambas cavidades, como se expresó en el Capítulo 4). Estos resultados son analizados mediante la PSD de las señales medidas en el dominio del tiempo, lo que permite determinar aproximadamente su composición espectral y la condición de ajuste del sistema. Se establece un esquema de valores de frecuencias de salida posibles en función de la relación entre longitudes $K$, para valores todos los valores posibles, y se realiza una pequeña comparación con las medidas obtenidas en cada caso $\left(L_{M}, 2 L_{M}\right.$ y $\left.3 L_{M}\right)$. Finalmente, en la Sección 5.3 se realiza una comparación teórico-experimental con los datos obtenidos para $2 L_{M}$ y $3 L_{M}$. Se comparan también los resultados numéricos y experimentales de todos los láseres implementados, encontrando una concordancia alta entre ellos. En esta comparación también se utiliza la eficiencia de conversión del sistema y se realiza una estimación de la densidad espectral de potencia de la emisión de salida, la cual depende principalmente de la respuesta en frecuencia de las FBGs.

\subsection{Descripción de la configuración experimental}

La configuración experimental del láser APM se basa en el esquema mostrado en la Fig. 4.4, el cual incluye un acoplador tipo WDM de FO monomodo 980/1550 nm que se emplea para acoplar el haz de bombeo (rama de $980 \mathrm{~nm}$ ) y a su vez extraer la emisión pulsada generada mediante la rama de $1550 \mathrm{~nm}$. De esta manera, la rama común del acoplador WDM es conectada a la $\mathrm{FBG}_{1}$ de la Fig. 4.4. Un láser de semiconductor (SDL Pump Module modelo SDLO-2500-125) se conecta adecuadamente para permitir el acoplamiento con una FO estándar y se usa para bombear el medio activo del sistema. Este láser de semiconductor emite en una longitud de onda próxima a 975,12 nm, con una potencia de salida máxima de $125 \mathrm{~mW}$ y presenta un ancho de línea de 0,5 nm. La cavidad principal es construida con un tramo de FO altamente dopada con Er $^{3+}$ (LIEKKI Er30-4/125) y 
se completa en su longitud con un tramo de FO fotosensible (donde las FBGs fueron grabadas), obteniendo una longitud total igual a $L_{M}=1,976 \mathrm{~m}$ (medida desde el centro de las FBGs, como mencionamos en el Capítulo 3). La FO dopada presenta un pico de absorción igual a $30 \mathrm{~dB} / \mathrm{m}$ para una longitud de onda de $1530 \mathrm{~nm}$, un diámetro de campo modal (bajo la condición monomodo, medido a $1 / e^{2}$ del máximo de intensidad) igual de 6,5 um a $1550 \mathrm{~nm}$, una apertura numérica de 0,2 y una longitud de onda de corte en el rango de longitudes de onda comprendido entre 800-980 nm (según especifica el fabricante). La cavidad auxiliar es implementada con un tramo de FO estándar del tipo SMF28, la cual es empalmada a la FO fotosensible de la $\mathrm{FBG}_{2}$. El otro extremo libre de FO estándar es cortado de forma perpendicular al eje longitudinal de la misma con el fin de generar un semiespejo cuya reflectividad es del orden de $4 \%$ (reflexión de Fresnel). El corte de la FO estándar que genera dicho dispositivo es realizado mediante un cortador de precisión, el cual es empleado usualmente en el proceso de empalme de distinto tipo de FOs. La calidad del corte puede ser verificada fácilmente mediante una imagen obtenida de una fusionadora de FO marca Ericsson, aunque generalmente se obvia este paso debido a que el cortador garantiza un calidad más que aceptable para esta aplicación. Las longitudes de ambas cavidades fueron medidas y ajustadas con un error del orden de $\pm 1 \mathrm{~mm}$. Como ya hemos mencionado en el Capítulo 4, cuando estas longitudes no son exactamente ajustadas (mediante la relación de longitudes establecida por la Ec. 4.8), la longitud de onda de la emisión de salida del sistema se ajusta de forma automática dentro del límite impuesto por la longitud de penetración de las FBGs (relacionado al perfil de la dispersión de las mismas), como describe en su trabajo Huang et al. [22].

\subsection{Mediciones experimentales}

Las características espectrales de las FBGs uniformes (marca O-ELAND) empleadas en esta configuración experimental fueron medidas por reflexión mediante un analizador de FBGs marca MicronOptics modelo SM125-500 el cual tiene un láser basado en una celda de acetileno que le brinda una resolución espectral del orden de 1 pm con una potencia 


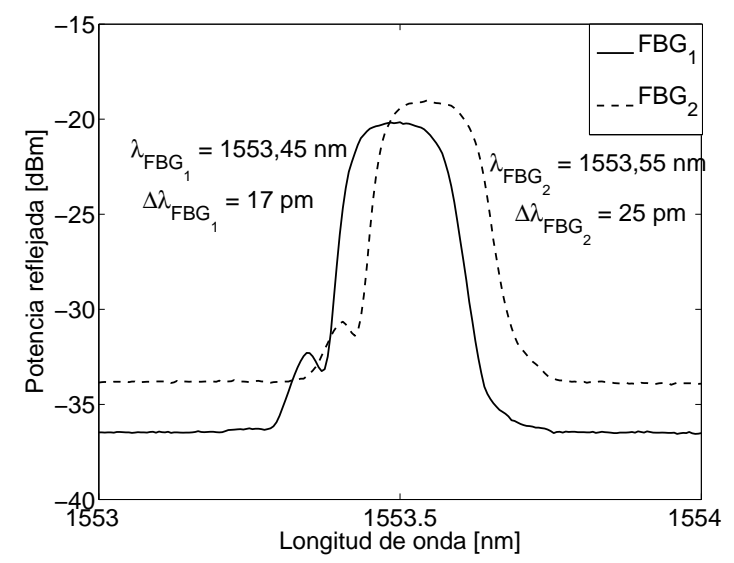

Figura 5.1: Caracterización espectral de las FBGs empleadas en la implementación del láser APM. La medida de la reflectividad de cada FBG se estima restando la potencia de la fuente al máximo valor de potencia reflejada.

aproximada de -7 dBm, y cuyos resultados se pueden observar en la Fig. 5.1. Como se puede observar, ambas FBGs presentan una longitud de onda próxima a $\lambda_{F B G} \approx 1553,5 \mathrm{~nm}$, el ancho de banda medido a mitad de la característica de reflexión es $\Delta \lambda_{3 d B} \approx 0,2 \mathrm{~nm}$ y los valores de reflectividad son próximos al $93 \%$ en ambos casos (se estima restando la potencia de la fuente). Estos resultados son similares a los obtenidos mediante un método de medida por transmisión empleando un analizador del espectro óptico OSA (Yokogawa AQ6370B) y una fuente de luz LED de banda ancha (de aproximadamente $40 \mathrm{~nm}$ ) y cuya longitud de onda está centrada en $1550 \mathrm{~nm}$, como se menciona en nuestro trabajo [2]. Los distintos métodos de caracterización espectral de las FBGs han sido discutidos e implementados en mi trabajo de tesis de grado [40].

En la Fig. 5.2 se puede observar la característica de transferencia del láser APM implementado. Esta se encuentra midiendo la potencia máxima de los pulsos para cada una de las distintas potencias de bombeo aplicadas, obteniendo una respuesta cuasi-lineal para un rango amplio de potencias de excitación. La característica mostrada en la Fig. 5.2 fue obtenida con el osciloscopio que mencionaremos a continuación, para una longitud de la cavidad auxiliar $L_{A}=0,395 \mathrm{~m}$, correspondiente a una relación de longitudes $K=5$, por lo 


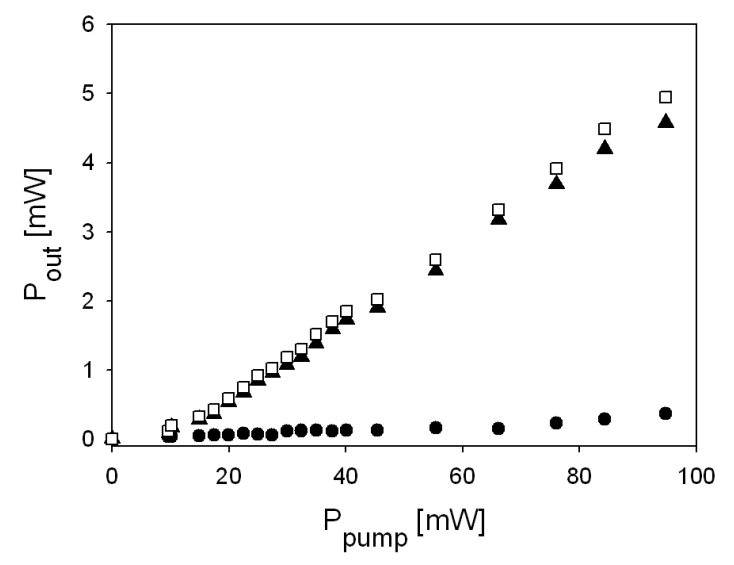

Figura 5.2: Salida del láser APM en función de la potencia de bombeo: • es la componente CW observada a la salida del láser, $\boldsymbol{\Delta}$ está relacionado al modo de operación pulsado y representa la potencia de salida del láser $(\mathrm{CW}+$ pulsado). La potencia del modo $\mathrm{CW}$ se obtiene calculando la diferencia entre el nivel de background y el nivel de fondo, obtenido cuando el fotodetector se encuentra apagado.

que la frecuencia de la emisión pulsada de potencia uniforme fue igual a $260 \mathrm{MHz}$ con un ancho temporal de los pulsos de 1,2 ns aproximadamente. Sin embargo, la característica de transferencia puede ser relevada mediante otras condiciones de ajuste del láser. Obviamente, esta caracterización se obtiene siempre para una condición de ajuste del láser, o sea, cuando la potencia de los pulsos generados es uniforme (no modulada). Como se puede observar en la Fig. 5.2, la emisión pulsada es obtenida cuando se supera un umbral de potencia pequeño (para potencias de bombeo mayores a $10 \mathrm{~mW}$ ). En general, la emisión de salida presenta un pequeño nivel de fondo o background sumado a la misma que está relacionado a la presencia del modo continuo CW en el sistema. Si la condición de ajuste no es satisfecha totalmente, dicho nivel puede ser incrementado, en detrimento de la ganancia del modo pulsado. Es importante notar que se obtuvieron trenes de pulsos estables y de potencia uniforme para un amplio rango de potencias de bombeo y aún empleando otros láseres de bombeo de mayor potencia (menores a $325 \mathrm{~mW}$ debido a limitaciones técnicas). Como ya hemos mencionado anteriormente en el Capítulo 4, un incremento sustancial de la potencia de las señales propagadas induce un incremento de los procesos no-lineales en las 
FOs y de los distintos dispositivos que conforman un sistema fotónico, induciendo cambios en la emisión pulsada de salida, tal como los observados en [28].

\subsubsection{Relaciones enteras de longitud}

La distribución temporal de los pulsos obtenidos a la salida del láser fue medida con un osciloscopio de muestreo LeCroy WaveExpert 100H que emplea un módulo óptico de $10 \mathrm{GHz}$ de ancho de banda. Con el fin de obtener una emisión pulsada de salida con potencia uniforme para diferentes frecuencias de repetición se utilizaron distintas longitudes de cavidad auxiliar $L_{A}$, manteniendo un valor de longitud de cavidad principal fija $\left(L_{M}=\right.$ 1,976 m). De esta forma, bajo la condición expresada en la Ec. 4.8, se obtiene una salida de potencia uniforme y estable mediante un régimen de operación APM. La frecuencia de la emisión láser a la salida puede ser expresada como un múltiplo de la frecuencia correspondiente a la cavidad activa, o sea $f_{\text {main }}=2 n_{\text {eff }} c / L_{M} \approx 52 \mathrm{MHz}$ para $L_{M}=$ 1,976 m. La Fig. 5.3 muestra las medidas de potencia instantánea obtenidas para distintos valores de $L_{A}$, expresados como: (a) 1,976 m, (b) 0,988 m, (c) 0,659 m y (d) 0,494 m (o bien $K=1,2,3$ y 4, respectivamente). Las frecuencias de repetición correspondientes fueron: $52 \mathrm{MHz}, 104 \mathrm{MHz}, 156 \mathrm{MHz}$ y $208 \mathrm{MHz}$, mientras que los anchos de pulso medidos en cada caso resultaron ser igual a: $T_{K=1}=3,5 \mathrm{~ns}, T_{K=2}=2,3 \mathrm{~ns}, T_{K=3}=1,6 \mathrm{~ns}$ y $T_{K=4}=$ 1,3 ns, respectivamente. En relación a los resultados obtenidos en [1], cada valor de $K$, expresado mediante un número entero, define una frecuencia de repetición que puede ser calculada mediante la ecuación $f_{\text {laser }}=K f_{\text {main }}$. En otros casos para los cuales el sistema no se encuentra adecuadamente ajustado, el régimen de operación APM no es admitido totalmente y prevalece el efecto de batido entre las señales ópticas que provienen de ambas cavidades del láser, lo que resulta en una salida cuya potencia no es uniforme o bien modulada y/o por momentos inestable (variable en el tiempo).

Un análisis complementario del modo pulsado, establecido para las características temporales observadas en la Fig. 5.3, puede ser desarrollado mediante la estimación de la PSD de dichas señales. En la Fig.5.4 se muestra la estimación de la PSD mediante la aplicación del 


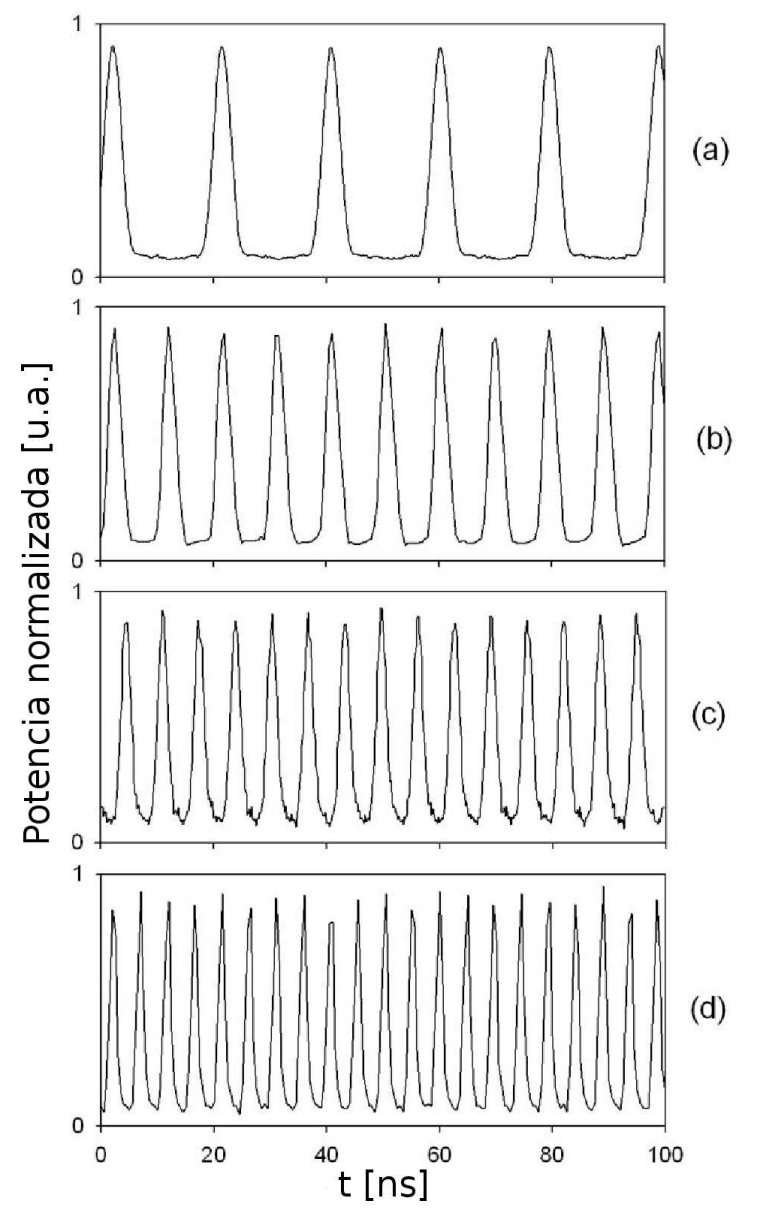

Figura 5.3: Emisión pulsada y estable del láser con potencia de salida uniforme, obtenida para diferentes valores de la longitud de la cavidad auxiliar $L_{A}$. (a) 1,976 m, (b) 0,988 m, (c) $0,659 \mathrm{~m} \mathrm{y}$ (d) $0,494 \mathrm{~m}$.

periodograma de Welch de cada una de las salidas temporales fotodetectadas. En la Fig.5.4 (a), para $K=1$, se observa que es complicado establecer un ajuste adecuado de las diversas componentes espectrales del láser, mientras que para las Fig.5.4 (b), (c) y (d), donde $K=$ 2, 3 y 4, respectivamente, las componentes espectrales resultan bien definidas a intervalos regulares de la frecuencia de repetición fundamental $\left(f_{\text {laser }}=m K f_{\text {main }}\right.$, donde $m$ es un entero). Esto se observa mediante el marcador "०", el cual fue empleado para representar los primero 50 armónicos. La condición $K=1$, presenta una componente intensa para 52 $\mathrm{MHz}$, mientras que algunas frecuencias, múltiplos de la fundamental, cumplen con la con- 


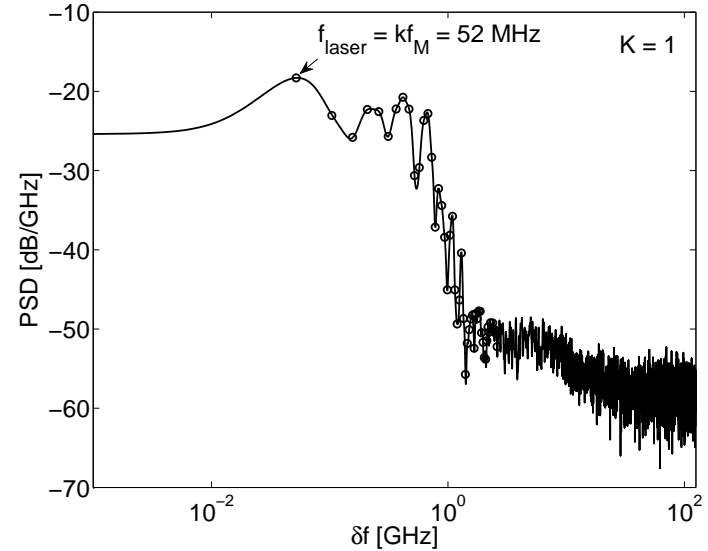

(a)

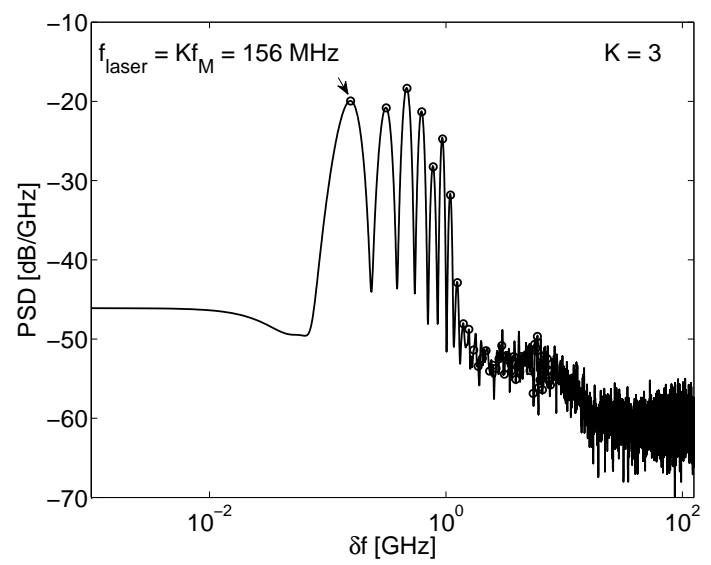

(c)

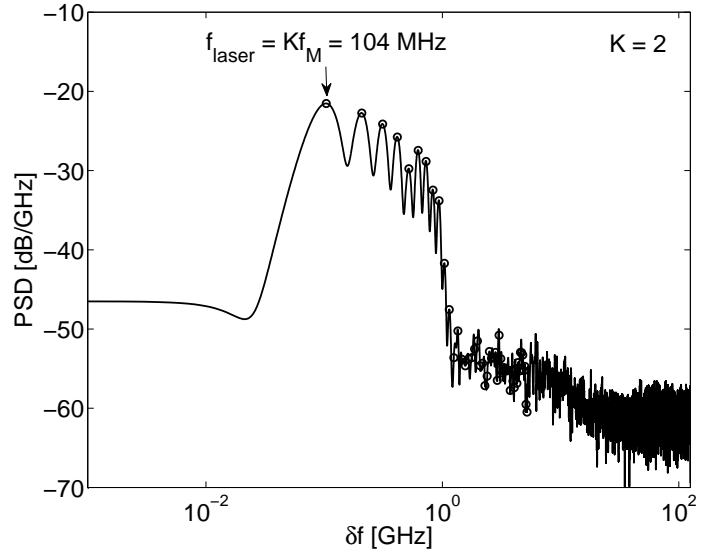

(b)

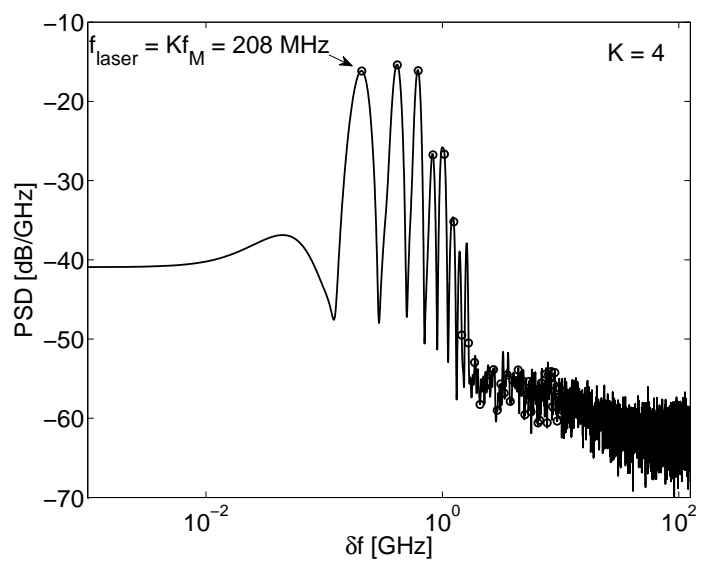

(d)

Figura 5.4: PSD para valores de $K$ enteros. Con "o" se marcan las frecuencias múltiplos de la frecuencia de salida que deberían aparecer en cada caso bajo un régimen de operación APM (presenta los primeros 50 armónicos). (a) $K=1$, (b) $K=2$, (c) $K=3$, (d) $K=4$.

dición mode locking y, otras no (batido de frecuencias). Dicha relación resulta siempre más compleja de hallar experimentalmente y de forma estable, como se podrá observar en la Sección 5.2.2, donde las frecuencias de salidas posibles se incrementan para valores $0<K<$ 1 (condición de ajuste más dificultosa de obtener en la práctica). En general, se observa que el número de componentes espectrales involucradas en todos los casos es menor a 20 (de distinta potencia), mientras que las componentes de alta frecuencia caen a un nivel de 


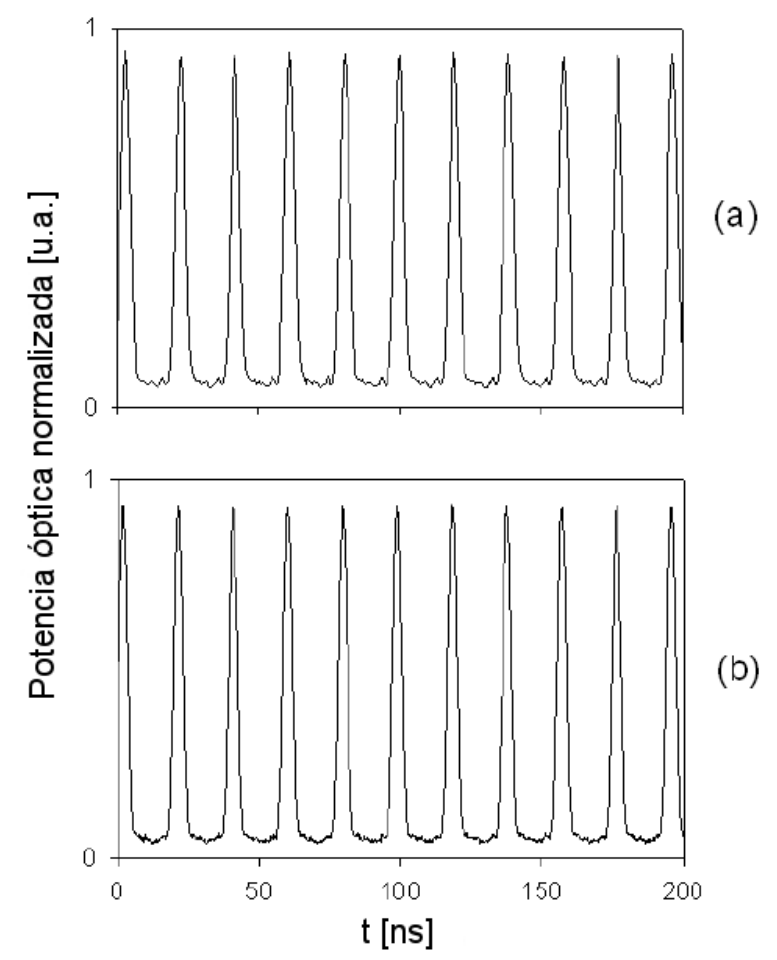

Figura 5.5: Emisión pulsada de salida para dos láseres APM con frecuencias de salida igual a $52 \mathrm{MHz}$. (a) $L_{M}=5,928 \mathrm{~m}, L_{A}=1,976 \mathrm{~m}(K=3)$. (b) $L_{M}=3,952 \mathrm{~m}, L_{A}=1,976 \mathrm{~m}$ $(K=2)$.

potencia semejante al ruido. Sin embargo, como podemos observar de las Figs. 5.4 (b), (c) y (d), el número de frecuencias que definen el ancho de banda medido a $3 \mathrm{~dB}$ del máximo no es mayor a 3 o 4 componentes. Esto verifica de alguna manera que el proceso de FWM es despreciable para $L_{A}<L_{M}$ con $K$ enteros, como veremos a continuación. Por otra parte, debemos notar que, para todos los casos aquí mostrados, y en los siguientes, hemos prescindido del valor medio de la señal, relacionado principalmente al modo CW. El filtrado de la componente de frecuencia $\delta f=0$, mediante el procesamiento digital de las señales fotodetectadas, se realiza para mejorar la estimación de las componentes espectrales de más baja frecuencia de la emisión láser generada.

Extendiendo la caracterización del láser implementado, construimos otro láser de similares características y empleando las mismas FBGs. El nuevo láser presenta una longitud 
de cavidad principal igual al doble de la longitud original, o sea $2 L_{M}=3,952 \mathrm{~m}$. Para implementar este nuevo sistema, que también funciona bajo el régimen APM, se agregó un tramo de FO dopada con $\mathrm{Er}^{3+}$ adicional, con las mismas características que las descritas anteriormente (LIEKKI Er30-4/125). De esta manera, la nueva frecuencia relacionada a la cavidad principal del sistema fue igual a $f_{\text {main }}=26 \mathrm{MHz}$, y las frecuencias de salida obtenidas por medio de un cambio en la longitud de la cavidad auxiliar verificaron la ecuación $f_{\text {laser }}=K f_{\text {main }}$ (donde $K=L_{M} / L_{A}$ es un número entero). Como resultado, se obtuvieron valores de frecuencia de salida iguales a $26 \mathrm{MHz}(K=1), 52 \mathrm{MHz}(K=2), 78 \mathrm{MHz}$ $(K=3), 104 \mathrm{MHz}(K=4), 130 \mathrm{MHz}(K=5)$, etc., observando que los anchos de pulso medidos son coincidentes a los obtenidos para el primer láser y para los mismos valores de frecuencia. En el mismo sentido se implementó un tercer láser que opera en régimen APM, cuya longitud de cavidad principal fue igual a $3 L_{M}=5,928 \mathrm{~m}$. Para ese caso, la frecuencia fundamental fue igual a $f_{\text {main }}=17,33 \mathrm{MHz}$ y se obtuvo una salida pulsada con frecuencias de: $17,33 \mathrm{MHz}(K=1), 34,66 \mathrm{MHz}(K=3), 52 \mathrm{MHz}(K=3)$ y $69,33 \mathrm{MHz}(K=4)$, nuevamente disminuyendo la longitud de la cavidad auxiliar $L_{A}$. Este procedimiento experimental fue empleado para determinar de forma aproximada el peso de otros procesos no-lineales en el sistema láser implementado. Uno de los procesos que cobra importancia cuando las longitudes de FO son incrementadas es el FWM, el cual en sí es un mecanismo de generación de nuevas componentes espectrales que cumplen una dada condición de fase. En general, el efecto de este proceso es importante en láseres de pulsos ultracortos, donde el proceso de XPM también debe ser tenido en cuenta, debido principalmente al gran ancho de banda que poseen dichos sistemas (como ya hemos mencionado en el Capítulo 4). El modo de operación APM es sensible al FWM, debido a que las componentes espectrales cumplen una condición de ajuste de la fase. Sin embargo, como se pudo observar de las medidas realizadas y el análisis mediante la PSD, el número de componentes espectrales involucradas es pequeño (generalmente menor a 3 componentes espectrales definidas por el ancho de banda de $3 \mathrm{~dB}$ ) por lo que, para las potencias de bombeo aplicadas es posible despreciar los efectos del FWM y la XPM. Una comprobación simple de esta afirmación se hace mediante la siguiente medida experimental: cuando $L_{A}=1,976 \mathrm{~m}$, se obtuvo una 
salida pulsada a $52 \mathrm{MHz}$ con un ancho de pulso igual a 3,5 ns para todos los láseres implementados (para distintos valores de $K$ en cada caso). Estos resultados se muestran en la Fig. 5.5, donde se observa que el ancho temporal de los pulsos depende únicamente de la longitud $L_{A}$ (para un mismo par de FBGs), la cual debe satisfacer la relación definida por la Ec. 4.8. De esta forma, para el rango de potencias de bombeo aplicadas, los láseres implementados mantienen sus propiedades tiempo-frecuencia invariantes.

\section{Discusión de resultados experimentales}

En general, mediante los resultados experimentales mostrados anteriormente y los que se podrán observar en la Sección 5.2.2, se puede verificar que una disminución en la longitud de la cavidad auxiliar $L_{A}$ (para $L_{M}$ fija) induce una disminución en los anchos de pulso observados, mientras que de una forma inversamente proporcional, la frecuencia de salida se ve incrementada. Aunque la dispersión de segundo orden introducida por la FO dopada con $\mathrm{Er}^{3+}$ es mayor a la observada en FO estándar, se puede suponer que la distorsión introducida en el dominio del tiempo es pequeña (para las longitudes empleadas y los anchos de pulso obtenidos). Esto se debe principalmente a que el ancho temporal promedio de los pulsos obtenidos es de algunos nanosegundos. Sin embargo, bajo otras condiciones del mismo láser se pueden obtener pulsos del orden de las centenas de picosegundos [1], o menores, del orden de los 10 ps, empleando una cavida auxiliar de aire [133]. En este sentido, un tópico de gran interés en el diseño y optimización de este tipo de sistemas está relacionado a los cambios de la dispersión del medio activo, en módulo y signo, en función de la longitud de onda de la emisión generada (definida en nuestro caso por las FBGs) [14]. Un cambio de la longitud de onda de la emisión generada dentro de la zona espectral comprendida entre 1530-1550 nm implica un cambio en el módulo y signo de la dispersión cromática, como ya hemos mencionado en el Capítulo 2, lo que puede significar una alteración notable de la respuesta temporal de los pulsos generados. En la Fig. 5.6 (a) se muestra la relación entre el ancho temporal de los pulsos y la frecuencia de repetición de los mismos. La línea de trazos indica un ajuste realizado a los valores experimentales mediante 


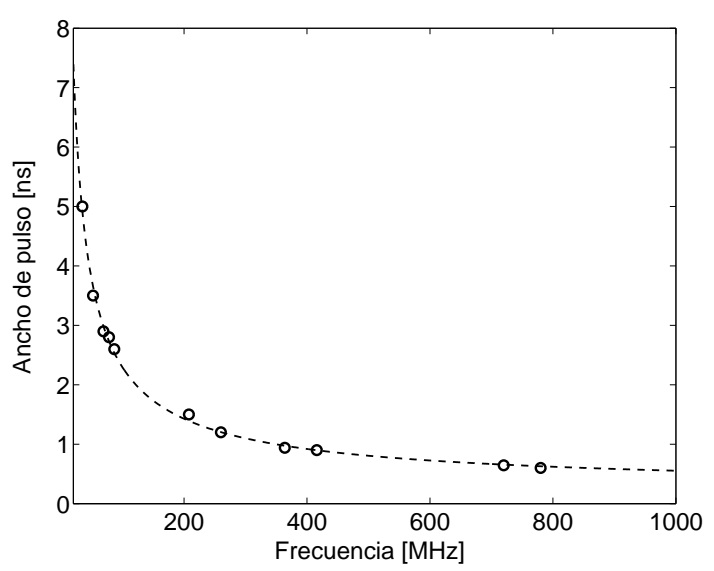

(a)

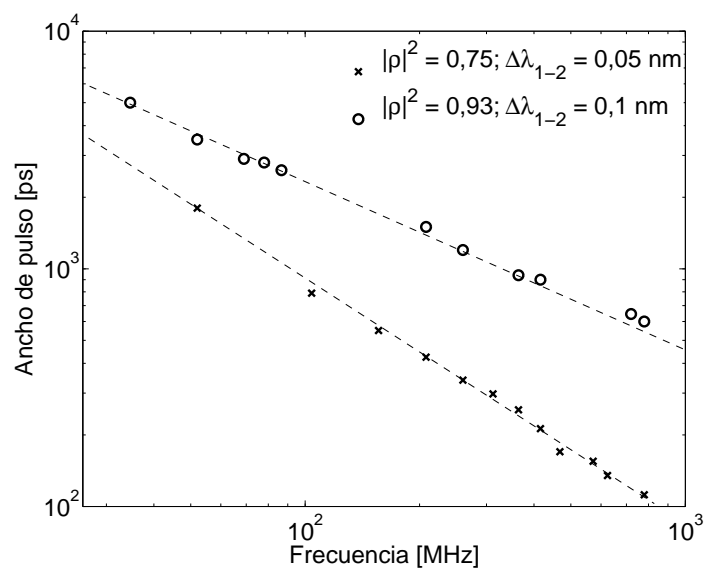

(b)

Figura 5.6: (a) Medición del ancho de los pulsos de salida en función de la frecuencia de repetición del láser APM. (b) Comparación entre los ajustes realizados en [1] (con $\times$, empleando FBGs provistas por la Universidad de Valencia, España) y los evaluados en nuestro trabajo [2] (con o, utilizando FBGs comerciales marca O-ELAND).

una aproximación del tipo $T_{\text {pulse }}=1,337 \times 10^{-3} f^{-0,7226}+1,045 \times 10^{-10}$ para un valor de reflectividad de las FBGs igual a $93 \%$, donde $T_{\text {pulse }}$ expresa el ancho temporal de los pulsos en unidades de segundos y $f$ representa a la frecuencia de salida en Hertz. Mediante el ajuste mencionado se podría inferir que el valor de $T_{\text {pulse }}$, para el láser implementado, está limitado a 105 ps aproximadamente.

Siguiendo la tendencia especificada en el trabajo de Russo et al. [1], el mismo sistema láser que funciona bajo un régimen APM, aunque construido con FBGs que presentan distintas características espectrales, puede alcanzar anchos de pulso menores (del orden de las centenas de picosegundos). Fundamentalmente, el efecto que producen las FBGs sobre la característica de la frecuencia de salida en función del ancho temporal de los pulsos puede ser observado en la Fig. 5.6 (b), donde las FBGs empleadas en [1] presentan dos modificaciones: por un lado, la reflectividad de las FBGs es menor (75\%), y por otra parte, la diferencia entre las longitudes de onda de Bragg entre la $\mathrm{FBG}_{1}$ y $\mathrm{FBG}_{2}$ es aproximadamente la mitad (aproximadamente $50 \mathrm{pm}$ ) del valor de la separación espectral 
obtenida en nuestro caso $\left(\Delta \lambda_{1-2}=\lambda_{F B G_{2}}-\lambda_{F B G_{1}} \approx 0,1 \mathrm{~nm}\right)$. Recordando el análisis estipulado para el estudio de la dispersión de la cavidad activa del láser APM, descrita por medio de la Fig. 4.17 (b), es posible inducir eficazmente un cambio en los valores de la dispersión de la cavidad principal de forma significativa (dentro del mismo orden de valores o bien mayor de la dispersión cromática, expresado anteriormente para el medio activo) alterando el ancho de los pulsos generados. Esta afirmación deberá ser discutida en trabajos futuros, aunque se apoya fuertemente en que las características temporales de las señales obtenidas dependen de la ubicación espectral relativa entre las FBGs (las cuales pueden ser modificadas mediante un cambio de la temperatura o una deformación local), como se pudo verificar empíricamente, entre otras posibilidades. Por otra parte, el incremento de los valores de reflectividad de las FBGs produce un incremento de la energía dentro de la cavidad activa, lo que se traduce en un posible aumento de la fase no-lineal y en una disminución de la intensidad de acoplamiento de los campos eléctricos provenientes de ambas cavidades del láser. En este sentido, un acoplamiento débil (campos con potencias menores al caso anterior) presenta una ventaja respecto a la disminución del ancho temporal de los pulsos.

Retomando la discusión de la implementación realizada en nuestro trabajo [2], debemos recordar que en la Fig. 5.6 (a) se incluyen los resultados experimentales de todos los láseres APM descritos anteriormente, con longitudes de cavidad principal resultaron igual a $L_{M}=$ $1,976 \mathrm{~m}, 2 L_{M}=3,952 \mathrm{~m}$ y $3 L_{M}=5,928 \mathrm{~m}$. En todos los casos observados, cuando la reflexión del semiespejo es aplacada (por ejemplo, mediante micro-curvaturas generadas en la FO de la cavidad auxiliar, o bien empleando un gel que adapta la diferencia de índices de la interfaz FO-aire), se observa que la emisión láser presenta una frecuencia de repetición la cual está únicamente relacionada a la frecuencia fundamental, definida por las propiedades de la cavidad principal del sistema. Esta comprobación práctica es de gran interés para conocer el estado de funcionamiento del láser APM implementado. 


\subsubsection{Generalización para distintas relaciones de longitud}

Un caso particular se puede encontrar para algunos valores de $K$ expresados como un número racional $\left(K=M / N>1\right.$, con $M$ y $N$ enteros y coprimos, donde $L_{M}>L_{A}$ ) [1]. En estos casos, la frecuencia de la emisión medida a la salida del láser se puede aproximar mediante $f_{\text {laser }}=M f_{\text {main }}$. De esta manera, para una $L_{M}=1,976 \mathrm{~m}$ y empleando FBGs como las utilizadas en [1], se pueden obtener emisión para $K=4 / 3,7 / 4$ y 12/7, como se observa en la Fig. 5.7. Sobre la izquierda se muestran las tres salidas en el dominio del tiempo y sobre la derecha sus respectivas PSDs, para las cuales el láser ha sido adecuadamente ajustado. En general, se puede observar que para un mismo valor de $K$ el sistema se encarga de elegir una emisión de salida uniforme, estable y de la menor frecuencia posible. Sin embargo, es posible obtener mayores frecuencias de repetición, aunque la señal fotodetectada presenta algún tipo de modulación. Este comportamiento es atribuido principalmente a que el número de frecuencias de repetición posibles, cerca de la condición $K=1$, se ve incrementado, como veremos a continuación.

Particularmente, hemos podido determinar otras emisiones pulsadas para valores de $0<$ $K<1$ (expresadas mediante la relación de números primos). En la Fig. 5.8 se observan diferentes salidas del láser para distintas longitudes de cavidad auxiliar empleadas: (a) $L_{A}=2,273 \mathrm{~m}$, (b) $L_{A}=2,189 \mathrm{~m}, \mathrm{y}$ (c) $L_{A}=2,073 \mathrm{~m}$. En estos casos, se puede observar una fuerte presencia de la componente fundamental del láser, establecida por la longitud de la cavidad principal $\left(f_{M}=52 \mathrm{MHz}\right)$ como una frecuencia moduladora, mientras que también se encuentran presentes otras estructuras de pulsos de menor duración temporal (con anchos de pulso promedio entre 500-600 ps). Es notable, en estos casos, la influencia de las diversas componentes espectrales en la determinación de las características temporales de la señal moduladora, la cual incrementa su efecto a medida que nos acercamos a la condición $K \approx 1$. Estos resultados también se ajustan a la característica del ancho temporal de los pulsos en función de la frecuencia de repetición anteriormente mostrada en la Fig. 5.6. En la Fig. 5.8 (a) se muestra la PSD de dicha señal, estableciendo las frecuencias: $52 \mathrm{MHz}$, $264 \mathrm{MHz}, 571 \mathrm{MHz}, 877 \mathrm{MHz}, 1.139 \mathrm{MHz}$ y una de potencia pequeña a 

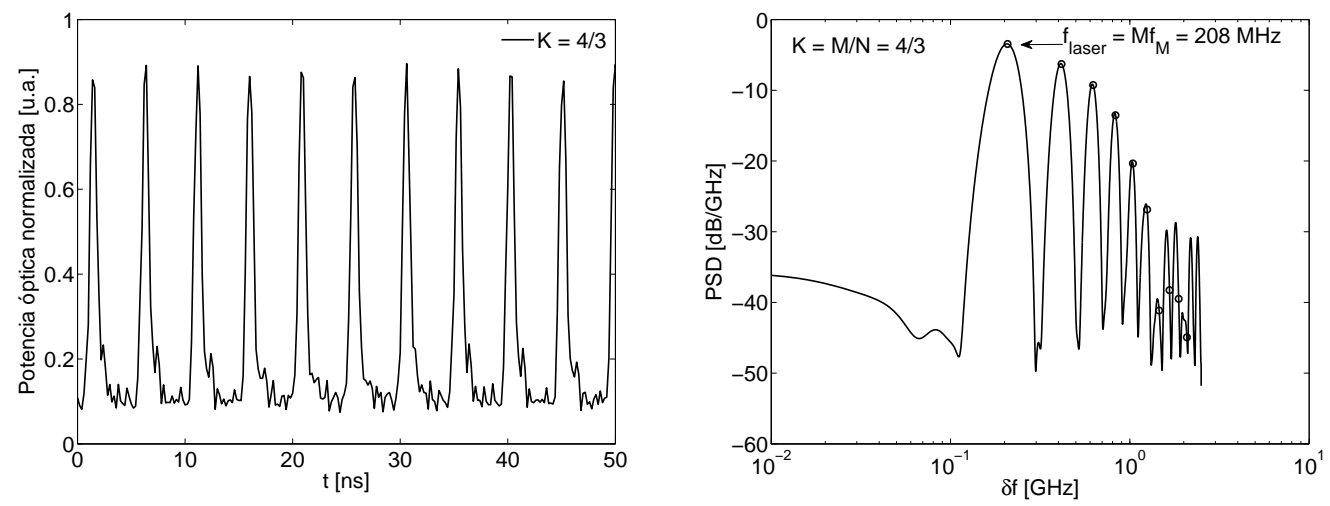

(a)
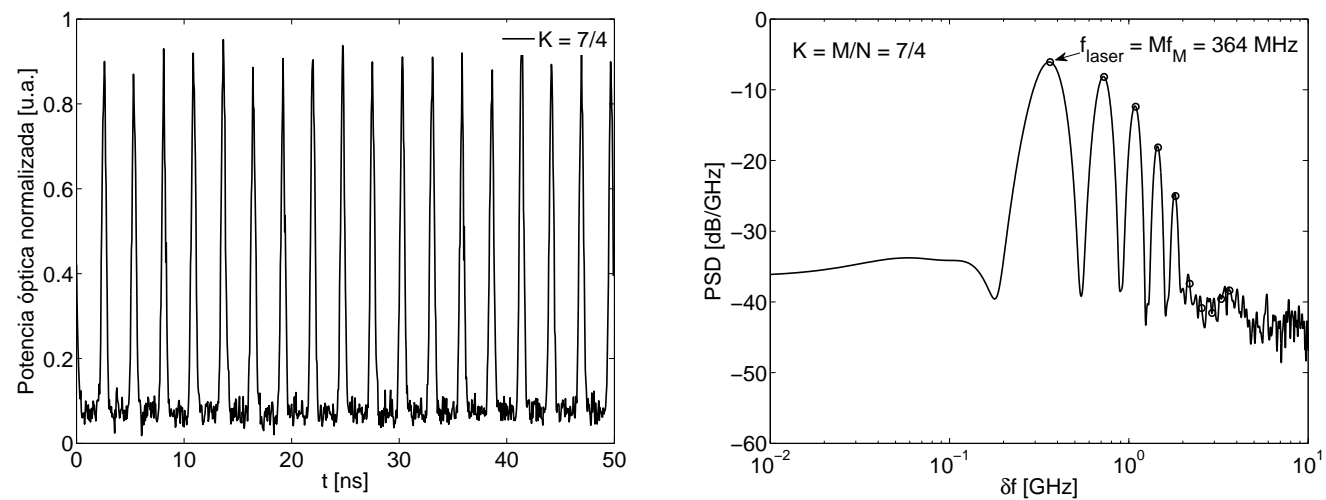

(b)
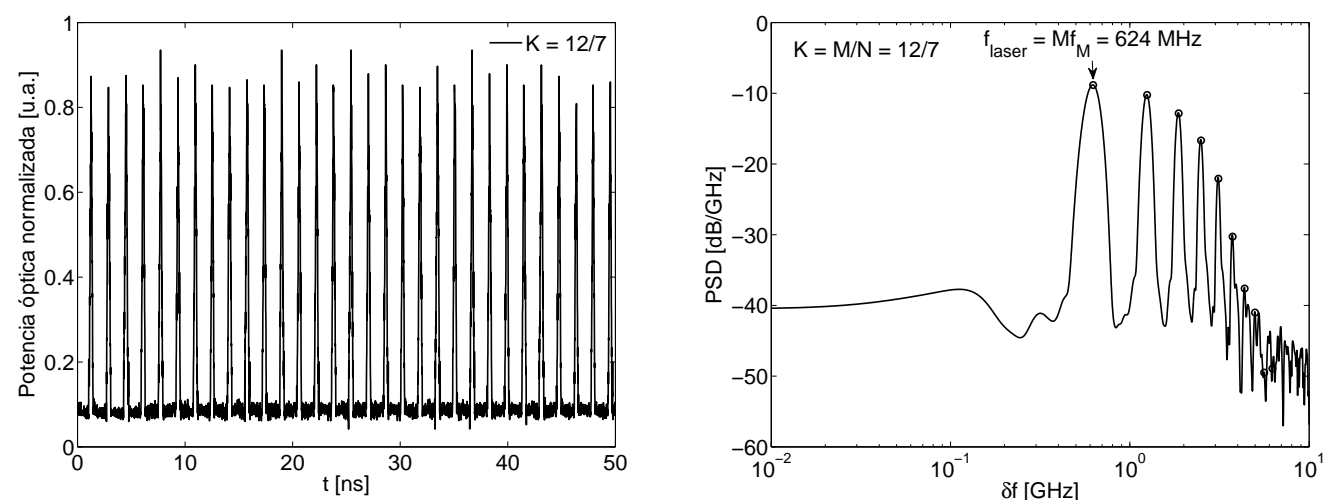

(c)

Figura 5.7: Salidas del láser para $1<K<2$ (relación de números coprimos). Sobre la izquierda se observan las mediciones temporales y del lado derecho la estimación de la PSD. (a) $K=4 / 3\left(f_{\text {laser }}=208 \mathrm{MHz}\right)$. (b) $K=7 / 4\left(f_{\text {laser }}=364 \mathrm{MHz}\right)$. (c) $K=12 / 7$ $\left(f_{\text {laser }}=624 \mathrm{MHz}\right)$. 
1.709 MHz. Suponiendo que la frecuencia fundamental es $f_{M}=52 \mathrm{MHz}$, las componentes espectrales restantes deberían aproximarse a un múltiplo entero de dicha frecuencia, como mencionamos anteriormente. De esta manera, la componente de $264 \mathrm{MHz}$ resulta en un factor 5 respecto de la fundamental y la componente de $571 \mathrm{MHz}$ en otro factor igual a 11 aproximadamente. Las demás componentes de frecuencia distan levemente de guardar una relación de número entero con la frecuencia fundamental. La relación de longitudes medida para este caso fue igual a $K=0,8693$, por lo que podría aproximarse a la relación 5/6 (que puede ser asociado a la componente de $264 \mathrm{MHz}$ ). Sin embargo, un valor igual a 11/13 (relacionado a $571 \mathrm{MHz}$ ) se aproxima mejor. Las demás componentes deberían comportarse de forma análoga, aunque la condición de ajuste para este caso no es óptima y los efectos del batido de las señales que provienen de ambas cavidades resultan ser más notorios. En la Fig. 5.8 (b) se puede observar las siguientes frecuencias: $52 \mathrm{MHz}, 466 \mathrm{MHz}, 983 \mathrm{MHz}$, $1.501 \mathrm{MHz}$ y una pequeña componente de $1.966 \mathrm{MHz}$. Nuevamente, la componente de 466 MHz se aproxima a un valor entero igual a 9, $983 \mathrm{MHz}$ resulta en un factor igual a 19 y las componentes restantes no guardan una relación bien definida con la frecuencia fundamental (aunque su efecto se puede despreciar debido a que son de potencia pequeña). La relación de longitudes medida para este caso fue igual a $K=0,903$, por lo que un valor racional probable igual a 9/10 (466 MHz) coincide muy bien con el obtenido de forma experimental. De todas formas, una relación 19/21 (983 MHz) también se ajusta a dicho valor de $K$, por lo que se supone que el láser funciona bajo una condición de funcionamiento probable, como veremos a continuación. La PSD observada en la Fig. 5.8 (c) muestra las siguientes frecuencias principales: $52 \mathrm{MHz}$ y $1.089 \mathrm{MHz}$, mientras que las demás componentes son despreciables en potencia. La componente de alta frecuencia permite obtener un valor racional igual a $21 / 22$, el cual se aproxima muy bien al valor real $K=0,953$, para una condición de ajuste cercana a $K=1$. Como veremos luego, los valores de frecuencia de salida posibles se extienden mayormente para $0<K<1$, por lo que una condición estable es más difícil de alcanzar (cambios muy pequeños de la longitud alteran significativamente la frecuencia de salida del láser).

Finalmente, podemos plantear un diagrama de frecuencias posibles establecidas mediante 

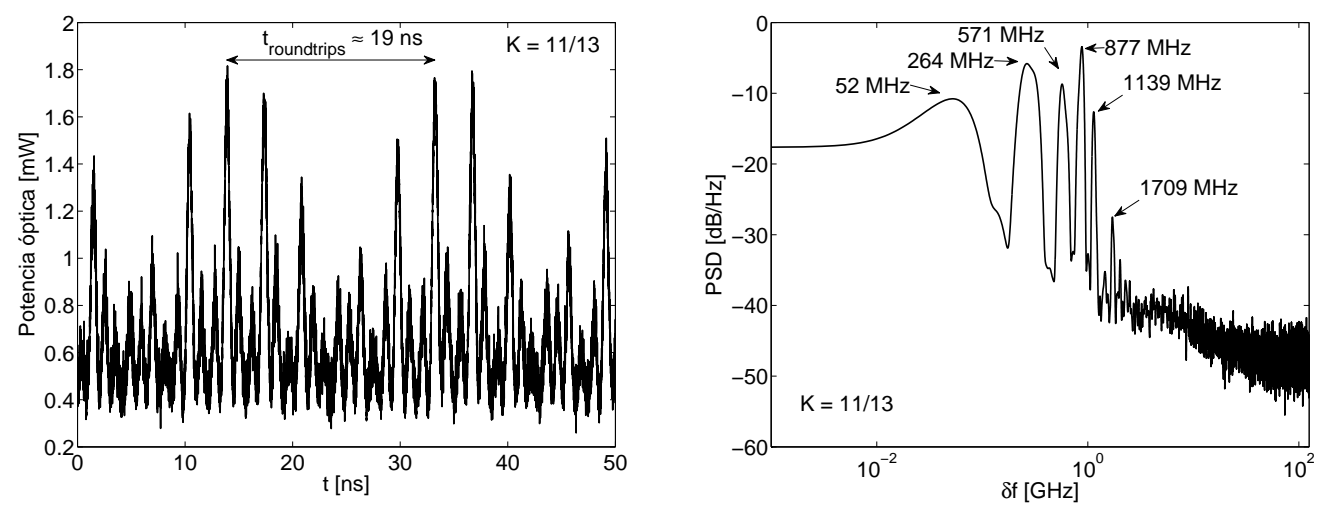

(a)
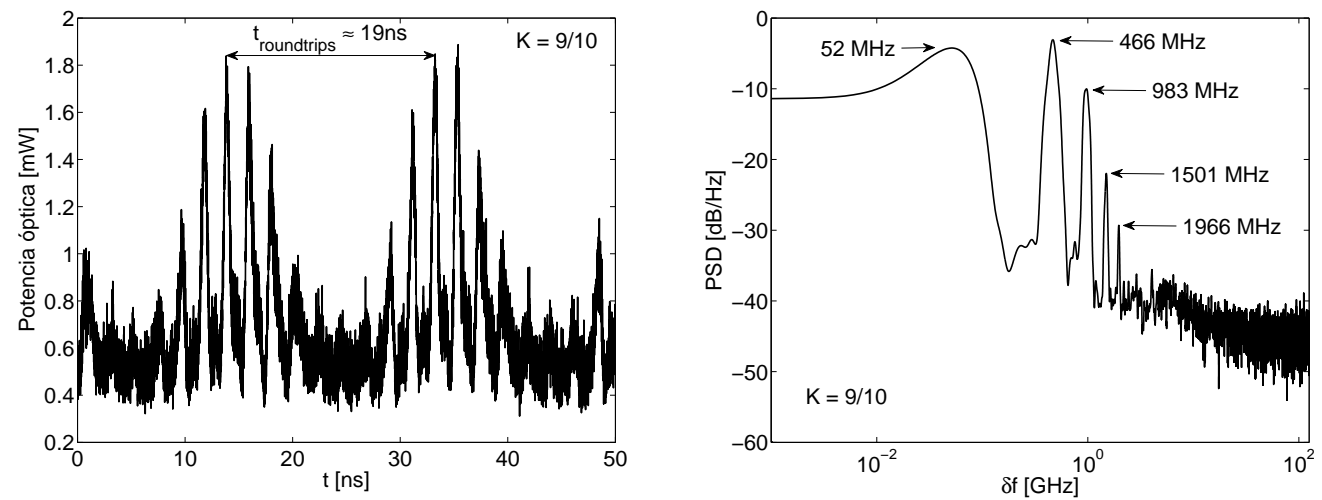

(b)
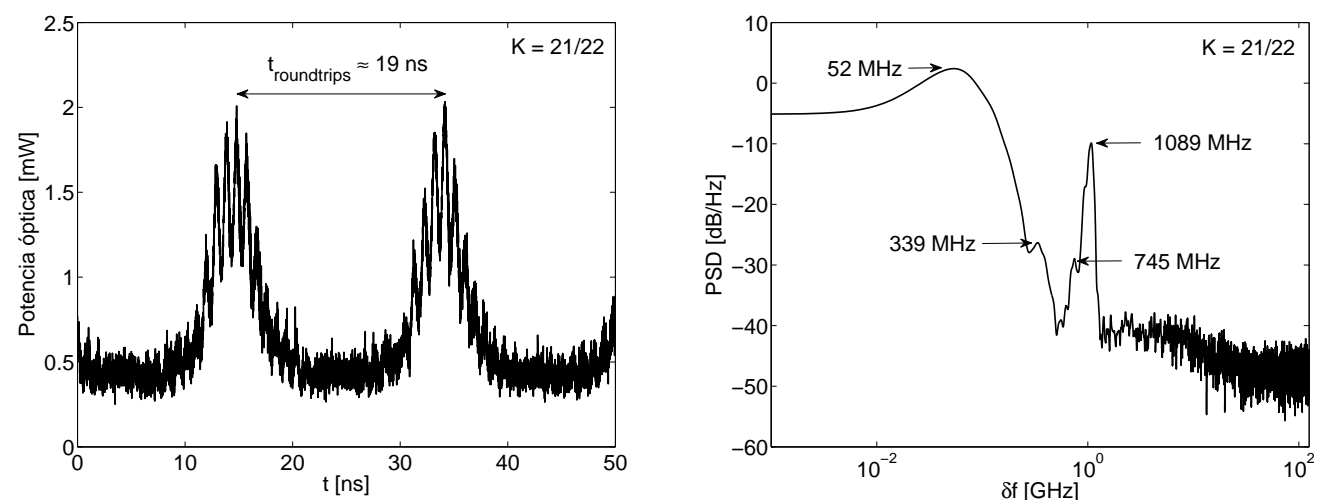

(c)

Figura 5.8: Potencias de salida del láser para $0<K<1$ (relación de números coprimos). Sobre la izquierda se observan las mediciones temporales. Del lado derecho, se muestra la PSD con las diversas componentes espectrales halladas en cada caso. (a) $K=11 / 13$ $\left(\bar{T}_{\text {pulse }}=477 \mathrm{ps}\right)$. (b) $K=9 / 10\left(\bar{T}_{\text {pulse }}=588 \mathrm{ps}\right)$. (c) $K=21 / 22\left(\bar{T}_{\text {pulse }}=500 \mathrm{ps}\right)$. 


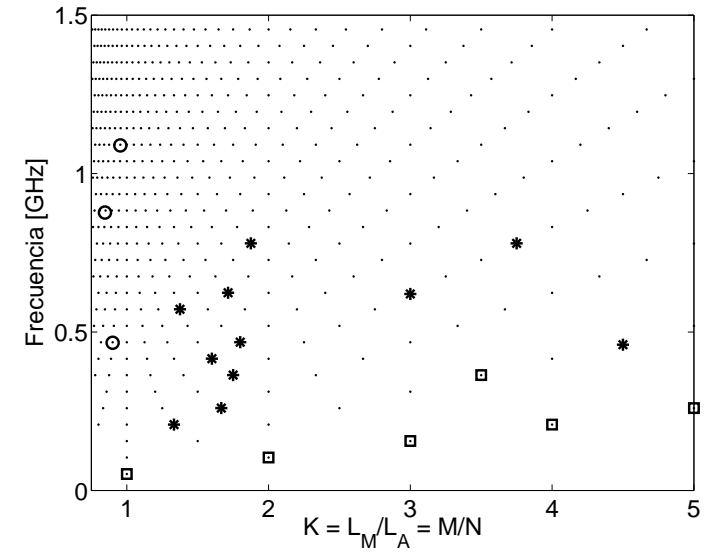

(a)

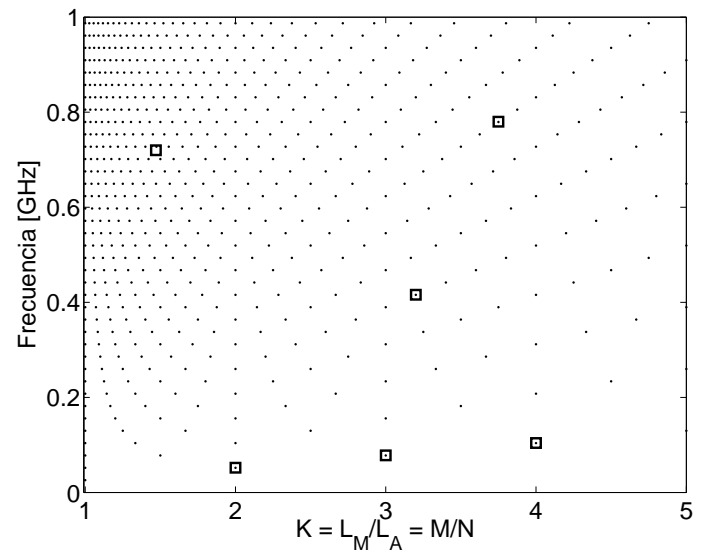

(b)

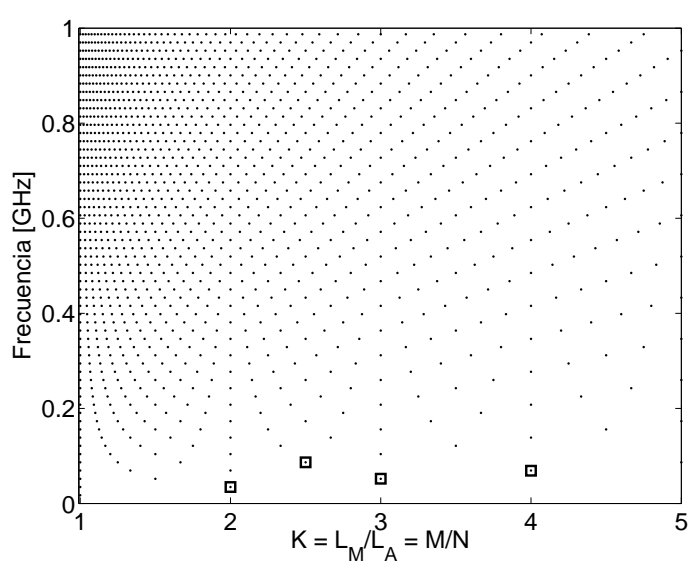

(c)

Figura 5.9: Frecuencias posibles y frecuencias medidas en función de $K$. (a) $L_{M}=1,976 \mathrm{~m}$ ( $\square$ salidas estable, $*$ salidas estables observadas en [1], o salidas moduladas en potencia). (b) $2 L_{M}=3,952 \mathrm{~m}$. (c) $3 L_{M}=5,928 \mathrm{~m}$. En general, el número de frecuencias posibles de estos diagramas se incrementa con la longitud $L_{M}$. Para valores enteros pares de dicha longitud $\left(2 L_{M}, 4 L_{M}\right.$, etc.) las frecuencias posibles para $2 L_{M}$ están incluidas en las frecuencias posibles halladas para $4 L_{M}, 6 L_{M}$, etc. Ídem para valores impares $\left(L_{M}, 3 L_{M}\right.$, etc.).

la Ec. 4.1 y el uso de la Ec. 4.8 que permite definir dichas frecuencias en función de la relación de longitudes $K$. De esta forma, en las Figs. 5.9 (a), (b) y (c) se expresan los distintos valores de frecuencias posibles para los tres láseres implementados (con longitudes 
de cavidad activa igual a $L_{M}=1,976 \mathrm{~m}, 2 L_{M}=3,952 \mathrm{~m}$ y $\left.3 L_{M}=5,928 \mathrm{~m}\right)$. En general, la grilla de frecuencias posibles en función de $K$ depende de la longitud de la cavidad activa, incrementando el número de frecuencias para longitudes mayores. A simple vista podemos observar que la grilla se hace más densa para $0<K<1$, lo que justifica el hecho de que el ajuste para esos valores de relación de longitudes sea mucho más complicado, como habíamos advertido. En particular, la grilla de frecuencias posibles para múltiplos pares, o sea $2 L_{M}, 4 L_{M}, 6 L_{M}$, etc., es coincidente (la correspondiente a $6 L_{M}$ contiene los puntos de $4 L_{M}$ y $2 L_{M}$, pero no se cumple la recíproca). De forma similar sucede con las longitudes expresadas como múltiplos impares.

\subsection{Comparación teórico-experimental de los resul- tados obtenidos}

Una forma de realizar una comparación entre los resultados numéricos y experimentales consiste en medir el ancho temporal de los pulsos a la salida del láser, como podemos observar en las Fig. 4.13 y Fig. 5.3. Los resultados numéricos obtenidos se ajustan muy bien a los experimentales correspondientes, mientras que la desviación máxima es de aproximadamente del $10 \%$. Esta conclusión podría ser demasiado optimista, debido a que los pulsos simulados en la Fig. 4.13 son libres de ruido. Sin embargo, y como ya hemos mostrado en el Capítulo 4 para el cálculo del régimen pulsado mediante un proceso de ruido, el promedio de varias realizaciones permite estimar dentro de la misma cota del error mencionada el ancho temporal de los pulsos. Debemos notar también que la ganancia del modo pulsado y modo CW son diferentes, por lo que el sistema de alguna manera filtra el ruido para que el modo pulsado prevalezca frente al modo CW (siempre y cuando se presente la condición de ajuste de la fase en el régimen mode locking). Esta afirmación pudo ser confirmada mediante las PSDs de las señales fotodetectadas, las cuales presentan en general una característica de baja frecuencia o pasabajos (para un ancho de banda mucho menor al establecido por las FBGs). 


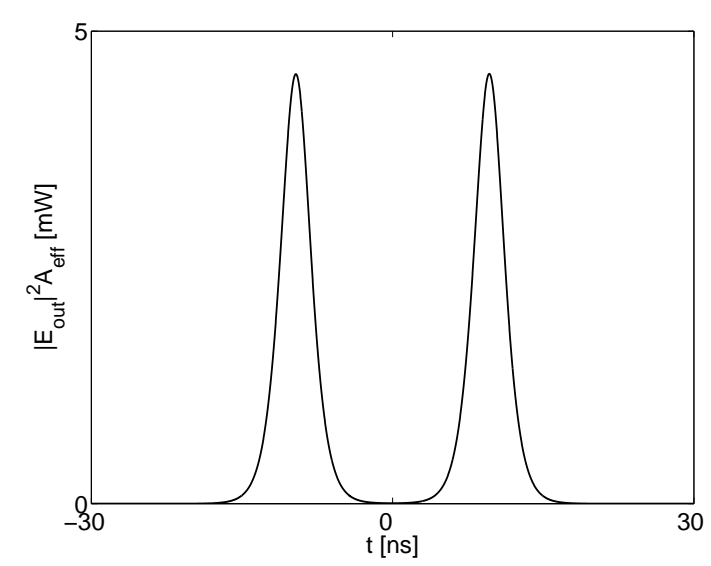

(a)

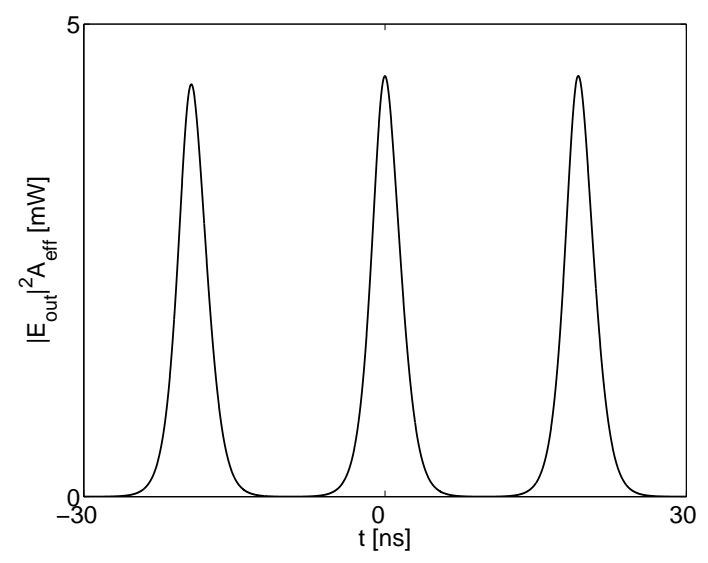

(b)

Figura 5.10: Simulación de la emisión pulsada del láser a una frecuencia igual a $52 \mathrm{MHz}$. (a) $2 L_{M}$ y $K=2$ (con ancho de pulso igual a $3,46 \mathrm{~ns}$ ); (b) $3 L_{M}$ y $K=3$ (con ancho de pulso igual a $3,48 \mathrm{~ns})$.

Extendiendo la longitud de la cavidad principal (formando dos láseres nuevos), como ya hemos mencionado a $2 L_{M}$ y $3 L_{M}$, se obtuvieron resultados teórico-experimentales similares. En ambos láseres hemos empleado una longitud de la cavidad auxiliar apropiada, a fin de obtener la misma frecuencia de salida, mostrando un comportamiento similar en cada caso. En las Figs. 5.10 (a) y (b) se muestran los resultados numéricos encontrados para la misma frecuencia de la señal de salida $(52 \mathrm{MHz})$, obtenidas con diferentes relaciones de longitudes $K$ y para los dos láseres mencionados (construidos con $2 L_{M}$ y $3 L_{M}$ ). Comparando estos resultados numéricos con los obtenidos de forma experimental, como muestra la Fig. 5.5, se establece que la diferencia entre dichos resultados es del orden del $1 \%$ para los valores de los anchos de pulso medidos. El error absoluto en las medidas observadas en las Figs. 5.3, 5.6 y 5.5 es menor que 50 ps, donde los errores de simulación para la mayoría de los casos verifican dicha cota. Como podemos observar, no se han obtenido cambios significativos en los anchos de los pulsos medidos a la salida del láser para la misma frecuencia de repetición. En este caso suponemos que, debido a que la magnitud de los anchos de pulso observados son del orden de los nanosegundos, la dispersión de la FO en el sistema juega un rol 


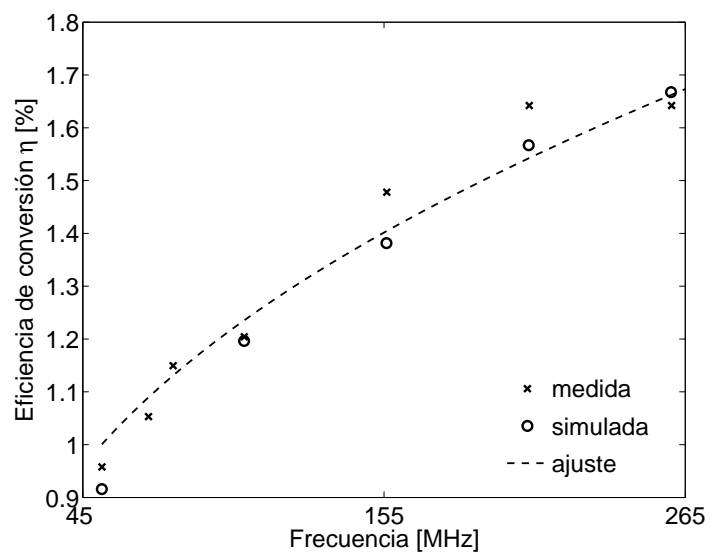

Figura 5.11: Eficiencia de conversión del láser en función de la frecuencia de salida, para los casos numéricos y experimentales analizados.

secundario. Sin embargo, como ya hemos mencionado, la dispersión puede incrementar su efecto en láseres de pulsos ultracortos.

La Fig. 5.11 se obtiene por medio de los valores de ancho de pulso en función de la frecuencia de salida medidos en la Fig. 5.6 (a), donde la eficiencia de conversión del láser se calcula como

$$
\eta=T_{\text {pulse }} f_{\text {laser }} \frac{P_{\text {pulse }}}{P_{\text {pump }}}
$$

donde $P_{\text {pulse }}$ y $P_{\text {pump }}$ son las potencias de pulso y excitación, respectivamente. Los valores obtenidos no son muy elevados, aunque pueden ser incrementados notablemente mediante la incorporación de algún mecanismo de modulación de las pérdidas en láseres APM, como ya hemos mencionado en los Capítulos 3 y 4 . Comparando con los resultados obtenidos de forma numérica, se puede realizar una aproximación de la eficiencia de conversión por medio de $\eta=1,421 \times 10^{-6} f^{0,4677}+4,352 \times 10^{-3}$, obteniendo una pequeña diferencia del orden del $3 \%$ entre las pendientes simulada y experimental. De esta forma, se puede inferir que el modelo numérico aplicado se ajusta de forma adecuada a los resultados medidos.

Finalmente, la densidad espectral de potencia (PSD) medida en cada uno de los láseres implementados, mediante el OSA anteriormente mencionado, se puede observar en la Fig. 5.12 


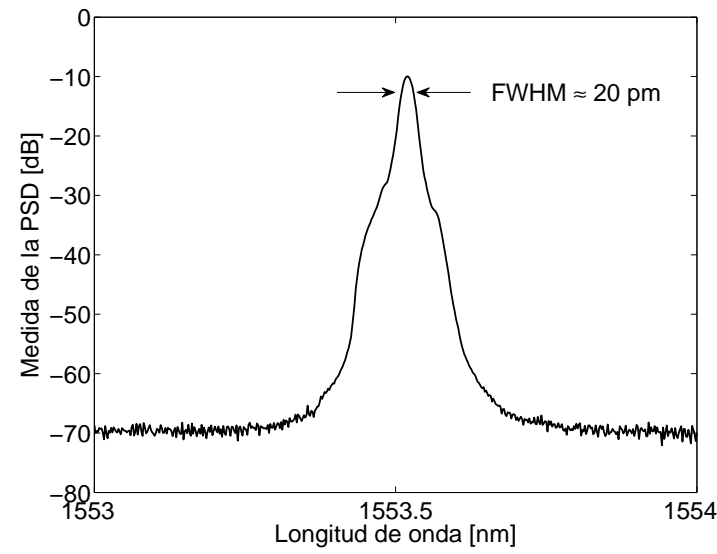

(a)

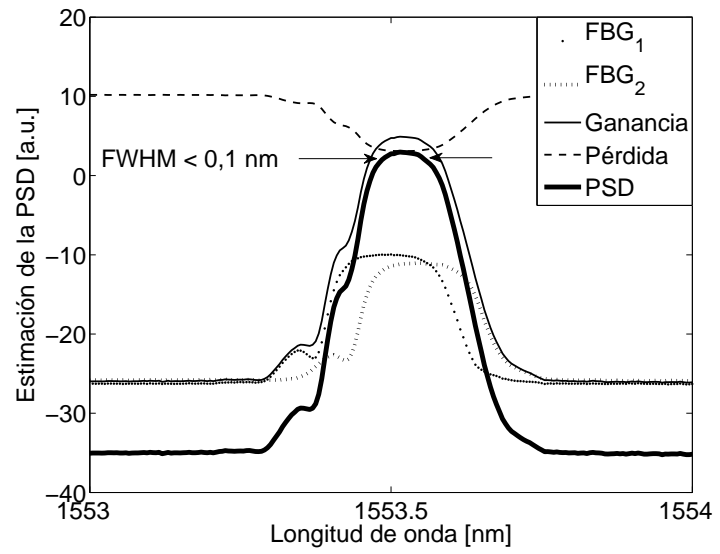

(b)

Figura 5.12: (a) Densidad espectral de potencia medida a la salida del láser APM. El ancho de banda de la emisión generada por el sistema láser implementado es FWHM $\approx 20 \mathrm{pm}$. (b) Estimación de la PSD mediante el uso de las características de reflexión de las FBGs mostradas en la Fig. 5.1. Se consideran los mecanismos de pérdida y ganancia de una cavidad Fabry-Perot para un nivel de excitación pequeño (resultando en la curva de ASE del medio activo filtrado por las FBGs).

(a). El ancho de banda de la emisión láser generada es de $20 \mathrm{pm}$ (alrededor de 2,5 GHz para una longitud de onda de trabajo cercana a los 1,55 $\mu \mathrm{m})$. Para todas las medidas realizadas anteriormente (diversas longitudes de cavidad y relaciones de longitudes $K$ ) no se ha podido establecer un cambio significativo de la medición de la PSD. Un ensanchamiento espectral (relacionado al efecto Kerr), obtenido mediante la aplicación del modelo numérico, no pudo ser verificado de forma experimental para los niveles de potencia de excitación establecidos. Sin embargo, y como hemos podido estimar de los datos experimentales observados, existe una modificación de las componentes espectrales de la PSD, dentro de un ancho de banda estimado en unidades de frecuencia menores a algunos gigahertz, como pudimos observar en la Sección 5.2.2. Por lo tanto, la razón de no tener resultados experimentales en este sentido se debe al límite impuesto por la resolución espectral del OSA. Aún obteniendo una resolución espectral diez veces menor a la especificada (la cual puede 
ser alcanzada por el OSA bajo ciertas condiciones), debemos notar que el solo cambio de una única muestra en la medición de esta nueva PSD, significaría el incremento del ancho de banda que hemos observado de forma teórica (mediante el modelo numérico). Por otra parte, se observa que el ancho de banda de la señal generada (medido a $3 \mathrm{~dB}$ del máximo de la PSD) es aproximadamente un $10 \%$ del ancho de banda establecido por las FBGs. En este sentido, la generación de una señal que presente un ancho de banda del especificado para estos dispositivos, suponiendo que las componentes espectrales verifican la condición de ajuste de la fase impuesta para el régimen de operación mode locking, permitiría obtener una emisión de salida en forma de trenes de pulsos ultracortos. Para que esto suceda, las diversas componentes espectrales de la señal generada deberían presentar un nivel de potencia relativamente uniforme (dentro de un ancho de banda definido por las FBGs). Una forma de modificar la característica espectral del sistema es mediante una adecuada elección de la longitud física de la cavidad auxiliar, como ya hemos visto en las simulaciones del Capítulo 4 y en las mediciones realizadas de forma experimental. En general, se observa que las señales generadas poseen una característica espectral pasabajos. Sin embargo, el incremento de la frecuencia de salida debida al aumento del valor de $K$ no modifica de forma significativa dicha característica. Una forma de ecualizar esta respuesta, con el fin de generar una especie de "peine" de frecuencias óptico, podría ser generando nuevas componentes mediante un proceso no-lineal como el FWM. Esto se logra, en principio, aumentando el número de componentes que verifican la condición de ajuste de la fase, por ejemplo, agregando un medio con un parámetro no-lineal elevado (incrementando el efecto Kerr). De otra forma es posible, disminuir la longitud de la cavidad activa con el fin de incrementar la frecuencia de repetición de los pulsos. Sin embargo, la longitud de la FO que forma la cavidad activa no puede ser arbitrariamente pequeña, debido a un límite práctico establecido por la máxima concentración del dopante (en nuestro caso el $\mathrm{Er}^{3+}$ ), el cual permite incrementar la energía de los pulsos mediante un aumento de la potencia de excitación de dicho medio (mecanismo de la ganancia).

En la Fig. 5.12 (b) se puede observar la estimación de la PSD correspondiente a la ASE filtrada por el sistema de realimentación (solapamiento espectral de ambas FBGs del láser). 
Como es de esperarse, al menos de forma intuitiva, esta característica es similar a la parte más ancha de la PSD observada en la Fig. 5.12 (a). Finalmente, para un nivel de excitación que supera al umbral del láser mostrado en la Fig. 5.2, la emisión estimulada obtenida presenta un ancho de banda más estrecho, correspondiente a la región espectral para la cual se supera el umbral láser donde FWHM $<0,1 \mathrm{~nm}$. 


\section{Capítulo 6}

\section{Conclusiones}

En este trabajo de tesis se llevó a cabo un estudio de los principales tópicos relacionados a la propagación de señales ópticas mediante un medio de transmisión no-lineal como la FO. El cálculo de dicha propagación mediante la ecuación no-lineal de Schrödinger, la cual es limitada a unos pocos términos, permite describir de una manera simple la propagación de pulsos gaussianos y de tipo secante hiperbólica bajo un régimen solitónico (régimen anómalo de la dispersión). El empleo de términos adicionales de la NLSE se basa principalmente en la posibilidad de realizar una descripción de sistemas de generación de pulsos de luz ultracortos, para los cuales es necesario emplear una mejor representación de los perfiles de la dispersión de cada uno de los elementos (dada por procesos tales como PMD y dispersión de alto orden) y de los procesos no-lineales relacionados al ajuste de fase coherente, como el FWM y la XPM, además del SRS y el self-steepening. Estos dos últimos deben ser introducidos en el cálculo de la propagación de pulsos con anchos de pulso menores a 1 ps. Sin embargo, en el ámbito de las temáticas tratadas en los Capítulos 4 y 5 de este trabajo de tesis, la descripción de la NLSE mediante los términos de atenuación y/o ganancia, dispersión de segundo orden y la automodulación de la fase, resultan ser suficientes en el modelado de pulsos cortos, como hemos podido comprobar a través de una comparación de resultados teórico-experimentales, la cual es desarrollada en el Capítulo 5. El modelado de diversos dispositivos fotónicos mediante el método de la matriz de trans- 
ferencia o TMM ha sido mostrado a través del diseño y caracterización de distintos tipos de redes de Bragg grabadas en FO. Además, el TMM ha mostrado una gran flexibilidad y potencial en la descripción de diversos sistemas y dispositivos, como los vistos en el Capítulo 3. En las aplicaciones relacionadas a la generación de luz coherente en distinto tipo de láseres, que operan en regímenes de Q-switch, como el desarrollado en la Sección 3.2.1, y mode locking en el Capítulo 4, como parte principal de este trabajo de tesis, el TMM ha mostrado ser una herramienta de análisis y diseño muy importante. Particularmente, el sistema láser que trabaja en régimen $Q$-switch permite obtener una señal óptica pulsada de gran potencia, del orden de los cientos de miliwatts y a una frecuencia de repetición de las decenas de Kilohertz o mayores, definida por un sistema de modulación [40]. En el mismo sentido, el diseño de sistemas interferométricos mediante el TMM, empleando acopladores de FO y FBGs, permitió estudiar los principios de interferencia y coherencia de las señales ópticas, los cuales son potencialmente aplicados a la modulación de las pérdidas en sistemas láser de pulsos cortos y ultracortos. Estos sistemas de modulación de la intensidad, producida mediante la modificación de una diferencia de camino óptico entre las ramas de un MI o MZI, son empleados en láseres que operan en régimen mode locking, con el fin de incrementar la intensidad y la frecuencia de repetición de los pulsos de salida, disminuyendo además los niveles de jitter presente en la fase de los mismos. Dichas cualidades son muy deseables en sistemas de sincronización electrónica aplicados al procesamiento digital de señales y en comunicaciones de alta capacidad [29,41], entre otras aplicaciones. El diseño de los parámetros físicos de las FBGs de período uniforme (longitud física y perfil del índice de refracción) y tramos de FO estándar, por medio del TMM, permitió analizar diversos dispositivos fotónicos, tales como estructuras DFB y cavidades Fabry-Perot, las cuales son aplicadas al diseño de láseres y filtros de canales WDM [39,40]. Estos dispositivos son compactos, pueden presentar diversas características espectrales, y al ser integrados en FO permiten eliminar los problemas de alineamiento producidos en sistemas análogos construidos con elementos de óptica tradicional. El procesamiento de señales ópticas mediante líneas de retardo puras o TTD, implementadas con FO estándar y FBGs como elementos de reflexión, es aplicado a un sistema de formación de haces de 
radiofrecuencias o beamforming, el cual es conectado a un PAA. Mediante un análisis de los principales parámetros de este sistema fotónico, tales como: i) la longitud física de los tramos de FO que definen el retardo introducido por las TTD, ii) longitud de onda de las FBGs y iii) longitud de onda del láser de semiconductor, se realiza un análisis estadístico de dichas variables, encontrando que el sistema presenta un estrabismo del haz de RF generado para pequeños cambios en la longitud de onda de las FBGs [43]. Otro factor que impacta fuertemente en el procesamiento óptico realizado con este tipo de sistemas son las no-linealidades introducidas por los distintos dispositivos de FO utilizados y por otros procesos como: la interferencia de segundo y tercer orden y el crosstalk producido por la interferencia de los lóbulos secundarios de las FBGs. El estudio de la propagación de los campos eléctricos en las FBGs de período no-uniforme, en particular de una LCFBG, fue aplicado en el diseño de un nuevo sistema de caracterización de señales ópticas de forma completa (intensidad y fase), con un error pequeño. El funcionamiento del mismo está basado en la implementación de una de las propiedades de la función de distribución de Wigner o WDF y en el empleo de una aproximación tiempo-frecuencia de los pulsos, relacionada a la pendiente del retardo de grupo de la LCFBG. El sistema resulta compacto mediante una configuración simple de dispositivos discretos de FO y emplea un par de fotodetectores de semiconductor para estimar las propiedades de la señal. Además, el sistema admite una amplia variedad de señales ópticas, y en particular, permite estimar trenes de pulsos cortos, del orden de 100 ps, para un valor de la pendiente del retardo de grupo igual a $\Phi_{20}^{r}=0,166 \mathrm{ps} / \mathrm{GHz}$, y trenes de pulsos ultracortos, para valores del coeficiente de modulación menores al especificado [44]. Sin embargo, la principal limitación de este sistema se presenta en el ancho de banda acotado de los fotodetectores, los cuales son generalmente menores a $40 \mathrm{GHz}$ para la banda $\mathrm{C}$ del espectro óptico, restringiendo el rango de utilización del sistema para trenes de pulsos con anchos temporales menores a 25 ps. El sistema basado en una LCFBG presenta dos limitaciones importantes: i) en general, el valor $\Phi_{20}^{r}$ depende en forma inversamente proporcional a la longitud física de la LCFBG, por lo que está limitado a longitudes pequeñas (menores a $1 \mathrm{~cm}$ ), ii) por otra parte, la distorsión o ripple en la característica del retardo de grupo de dicho dispositivo 
introduce un pequeño error sistemático en la estimación de la intensidad y la fase de las señales ópticas. Sin embargo, se han obtenido muy buenos resultados empleando un tren de pulsos del tipo secante hiperbólica y chirp lineal a la entrada del sistema, los cuales son similares a los generados con un láser de FO de cavidades acopladas, como el mostrado en la Sección 4.3. En este sentido, la estimación de la fase de los pulsos generados por estos sistemas láser, que funcionan bajo el régimen de operación mode locking, es un motivo para la implementación real del sistema de caracterización, lo que permitiría contrastar los resultados numéricos del perfil de la fase de los pulsos con sus análogos fotodetectados. Luego, una solución a las limitaciones mencionadas (valores pequeños de $\Phi_{20}^{r}$ y ripple del retardo de grupo), puede ser parcialmente abordada empleando FOs compensadoras de la dispersión o DCF.

En el marco de los mecanismos de generación de emisión estimulada coherente en forma de pulsos de luz, mediante láseres de FO, el estudio de sistemas que operan en régimen APM ha permitido describir el comportamiento en un láser de dos cavidades acopladas, el cual emplea como elementos constitutivos del sistema de realimentación un par de FBGs y un semi-espejo. En este caso, y como hemos mencionado en reiteradas oportunidades en los Capítulos 4 y 5, el régimen de operación mode locking admite diversas componentes de frecuencia, definidas por las longitudes de ambas cavidades, las cuales son sumadas a través de un mecanismo de pulsos que se propagan por el sistema y se acoplan de forma coherente sobre una FBG, común a ambas cavidades del láser. Se debe notar, que bajo una relación de longitudes físicas $K=L_{M} / L_{A}$ no entera o racional, la condición de ajuste de la fase no es verificada y la salida resulta modulada en intensidad, comportándose de forma análoga a un proceso de batido de las señales ópticas generadas. El modelo analítico propuesto para resolver el campo eléctrico de salida fue implementado mediante el uso de un operador no-lineal, basado en la NLSE, que tiene en cuenta los siguientes factores: pérdida o ganancia saturada dependiendo del tipo de FO evaluada (estándar o dopada, respectivamente), dispersión de segundo orden de cada una de las FO y los parámetros no-lineales. La justificación del empleo de dichos términos se basa en que la salida de este tipo de láseres presenta generalmente una solución solitónica [53,54], donde la dispersión de segundo orden 
y la no-linealidad trabajan de forma conjunta, como pudimos mostrar en las Secciones 2.2.2 y 4.3. Por otra parte, los procesos de pérdida de la FO estándar (cavidad auxiliar) y de la ganancia saturada de la FO dopadas con $\mathrm{Er}^{3+}$ (cavidad activa del láser), deben ser tenidos en cuenta en el modelo para una mejor representación del mismo. En particular, el mecanismo de la ganancia saturada definida en [14,32], aplicada en nuestro modelo analítico y numérico, se basa en el supuesto de que el medio activo no logra despoblarse por completo en tiempos cortos, como los observados a lo largo de este trabajo, y de forma completamente contraria a lo que sucede con el sistema láser implementado en la Sección 3.2.1. El estudio de los términos de la ecuación del sistema mediante dichos factores, una aproximación de los elementos de realimentación y el empleo de una herramienta de representación simbólica permiten obtener el campo eléctrico a la salida del láser, al menos de forma aproximada para valores pequeños de dispersión de las FBGs y para un conjunto de parámetros físicos relacionados a los distintos componentes del sistema. En rigor, dichos parámetros deberían ser estimados mediante diversas caracterizaciones experimentales, como por ejemplo, el valor de la dispersión cromática de la FO dopada con $\mathrm{Er}^{3+}$ y de las FBGs (cuyo perfil espectral de reflexión ha sido caracterizado adecuadamente, como se muestra en la Sección 5.2). Estas caracterizaciones podrían ser realizadas de forma relativamente simple mediante métodos del tipo low coherence interferometry (LCI), empleando fuentes de luz de emisores súperluminiscentes y un MI de FO [134], de una forma similar a los sistemas diseñados en la Sección 3.2.2. De todas formas, el conjunto de los parámetros propuestos, determinados a partir de distintas fuentes bibliográficas, ha resultado más que adecuado para la obtención de los resultados numéricos mediante un algoritmo que resuelve el sistema de ecuaciones definidas de forma analítica. Este algoritmo introduce el perfil completo de las FBGs, en módulo y fase, y permite calcular la propagación de los campos eléctricos mediante el SSM de paso variable con un error de fase menor a $1 \times 10^{-4} \mathrm{rad}$, mientras que el acoplamiento de los campos en la $\mathrm{FBG}_{2}$ (Fig. 4.4), común a ambas cavidades, se realiza a través del TMM. Con este modelo numérico se estudió el proceso de self-starting del láser mediante un proceso de ruido gaussiano, sumado a la semilla (pulso del tipo secante hiperbólica), presentando un pequeño valor de relación señal ruido a la entrada $\left(\mathrm{SNR}_{\text {in }}=\right.$ 
$0,1 \mathrm{~dB})$. Se pudo observar que el sistema se comporta de dos maneras diferentes, mediante dos valores de ganancia diferentes relacionados con cada uno de los modos de operación (pulsado y CW), alcanzando una relación señal ruido $\mathrm{SNR}_{\text {out }}=10 \mathrm{~dB}$. Mejores valores de $\mathrm{SNR}_{\text {out }}$ se pueden obtener incrementando la potencia de excitación del medio activo, mientras que el nivel de background de la señal resulta inalterado (puede ser modificado disminuyendo el umbral del láser). Mediante este nuevo modelo numérico se obtuvieron resultados que permiten describir la salida del láser para distintos valores del parámetro $K$, expresado como un número entero positivo mayor o igual a la unidad. Además, por medio de un análisis realizado mediante la estimación de la PSD de las señales generadas de forma numérica, se pudo determinar el número de componentes espectrales que intervienen en el proceso mode locking, el cual es generalmente un número pequeño $<3$, para un ancho de banda medido a $3 \mathrm{~dB}$ del máximo de potencia. El estudio de la dispersión en la cavidad activa del láser permite determinar la influencia de las características espectrales de las FBGs en las pérdidas de la cavidad (reflectividad de las FBGs) y en los cambios en el perfil de dispersión. Estos últimos resultan ser prácticamente despreciables empleando una FO dopada con $\mathrm{Er}^{3+}$ en una longitud de onda de trabajo $\lambda_{0}=1553,55 \mathrm{~nm}$, mientras que se producen cambios significativos en la dispersión cromática de dicha cavidad cuando el corrimiento espectral relativo entre las FBGs, definido por $\left(f_{\text {Bragg }_{2}}-f_{\text {Bragg }_{1}}\right) / f_{\text {Bragg }_{1}}$ (Fig. 4.17(b)), esta próximo a la mitad del ancho de banda de dichas FBGs. El coeficiente de reflexión definido para el subsistema de la cavidad auxiliar del láser, compuesta por la $\mathrm{FBG}_{2}$, la FO estándar y el semiespejo, permite estudiar el incremento de la fase no-lineal cuando el valor de $\left|\rho_{\text {mirror }}\right|^{2}$ es incrementado. En este sentido, respecto a las posibles inestabilidades del sistema, el modelo numérico presentado permite estimar un valor de cota máximo de la fase $\Delta \Phi<6 \times 10^{-3} \mathrm{rad}$, para la cual el láser mantiene una salida estable o de intensidad uniforme, para valores de $\left|\rho_{\text {mirror }}\right|^{2}<0,6$. Un comportamiento similar se ha podido observar de forma experimental, empalmando la FO estándar de la cavidad auxiliar a un colimador y empleando un espejo de alta reflectividad, obteniendo señales moduladas en potencia y ancho de pulso (condición inestable). Mediante los resultados experimentales obtenidos para salidas estables del láser de FO de dos cavidades acopladas, cuya implemen- 
tación y caracterización se mostraron en el Capítulo 5, hemos verificado la característica del ancho temporal de los pulsos de salida en función de la frecuencia de repetición de los mismos, para distintos valores de $K$ (enteros y racionales). Se realizó una comparación de los resultados experimentales observados en [1] y [2], y se planteó una hipótesis relacionada con el cambio de la pendiente de dicha característica, la cual se debe principalmente al empleo de FBGs con diferentes perfiles espectrales de reflexión (reflectividad y corrimiento espectral de las mismas, lo que induce, como mencionamos, una alteración de la respuesta de la dispersión). Esta hipótesis pudo ser verificada de forma empírica, mediante la aplicación de una pequeña presión sobre la $\mathrm{FBG}_{2}$, aunque una caracterización completa de estos cambios se deja para estudios futuros. Por otra parte, se realizó un análisis de las señales fotodetectadas mediante el uso del periodograma de Welch [131], que permite estimar la PSD de dichas señales, encontrando una muy buena correlación entre los resultados teóricos y experimentales. Además, se implementaron dos nuevos láseres con longitudes de cavidad principal igual a $2 L_{M}$ y $3 L_{M}$, con el fin de comprobar el peso de otros procesos no-lineales como el FWM. Encontramos que éste último no presenta mayor importancia para potencias de bombeo menores a $P_{\text {pump }}<325 \mathrm{~mW}$ y que el número de componentes espectrales involucradas en el proceso mode locking es pequeño (menor a 3 en un ancho de banda medido a $3 \mathrm{~dB}$, verificando los resultados numéricos mostrados en la Sección 4.3.2). De esta manera, los datos medidos de la frecuencia de repetición y el ancho temporal de los pulsos en estos nuevos láseres se corresponde muy bien con la característica obtenida anteriormente para una longitud de cavidad principal igual a $L_{M}=1,976 \mathrm{~m}$. De esta manera, todas las medidas realizadas pueden ser resumidas en un diagrama de frecuencias posibles en función de la relación de longitudes expresada por el parámetro $K$, mientras que se han observado bajos niveles de ruido en los pulsos fotodetectados (verificando la hipótesis planteada en la Sección 4.3.1). Además, se realizó una comparación teórico-experimental para valores de $K$ enteros, mediante la eficiencia de conversión del láser, encontrando que dichos resultados se ajustan con un error pequeño, menor al 10\%. En todos los casos analizados, la medición de la PSD resultó sin cambios significativos debido a una limitación práctica impuesta por el OSA. Sin embargo, una estimación de la misma mediante el perfil espectral 
de las FBGs resulta conveniente para una potencia de excitación próxima al umbral del láser $\left(P_{\text {pump }}=10 \mathrm{~mW}\right)$, donde la característica queda definida aproximadamente por la emisión espontánea amplificada o ASE, la cual es filtrada por el sistema de realimentación. Finalmente, y como resumen de las líneas de investigación que se desean seguir en un futuro cercano, se encuentran: i) mejoras en las características del sistema láser propuesto, relacionadas al incremento de la frecuencia de repetición (disminución del ancho temporal) de los pulsos (mediante el empleo de sistemas de modulación intracavidad y/o de forma externa); ii) desarrollo de nuevos algoritmos basados en el SSM y el TMM, orientados a describir la propagación en láseres de pulsos ultracortos que funcionan bajo el régimen de operación mode locking, los cuales deberían admitir el modelado de otros procesos nolineales tales como el FWM, XPM y algunos efectos de scattering inelásticos, dependiendo del caso; iii) implementación real del sistema de caracterización de pulsos propuesto, con el fin de estimar la fase de los sistemas láser anteriormente mencionados y en general para otro tipo de señales ópticas; iv) estudio y diseño de aplicaciones de los láseres APM en sistemas de comunicación ópticos, metrología y maquinado de materiales. 


\section{Apéndices}

\section{Apéndice A: Aplicación del método SSM al calculo de la NLSE}

La propagación expresada a través de la Ec. 2.5 es una ecuación diferencial no-lineal a derivadas parciales, la cual no resulta generalmente en una solución analítica, excepto para algunos casos específicos en los cuales el método de scattering inverso puede ser aplicado [135]. Por lo tanto, una aproximación numérica es necesaria para entender los efectos no-lineales en las FOs. Existe una amplia gama de métodos que son empleados para dicho propósito, los cuales pueden ser clasificados en dos grandes grupos: los métodos de diferencias finitas y los métodos pseudo-espectrales. Estos últimos son generalmente hasta un orden de magnitud más veloces para conseguir la misma precisión. Un método que ha sido empleado de forma extensa para resolver el problema de la propagación de pulsos en un medio dispersivo es el SSM [136]. La velocidad relativa de este método, comparada con la mayoría de los métodos de diferencias finitas puede ser atribuida en parte al empleo del algoritmo de la transformada rápida de Fourier (FFT) [131].

La filosofía detrás del SSM se basa en reescribir la NLSE, discriminando de alguna manera la parte lineal de la no-lineal, como se muestra a continuación

$$
\frac{\partial E}{\partial z}=\left(\hat{O}_{D}+\hat{O}_{N L}\right) E
$$

donde $\hat{O}_{D}$ es un operador diferencial de la dispersión y la absorción de un medio lineal, mientras que $\hat{O}_{N L}$ es un operador no-lineal. Estos operadores quedan definidos por medio 
de la NLSE anteriormente definida como

$$
\begin{gathered}
\hat{O}_{D}=-\frac{\alpha}{2}-\frac{i}{2} \beta_{2} \frac{\partial^{2}}{\partial t^{2}}+\frac{1}{6} \beta_{3} \frac{\partial^{3}}{\partial t^{3}} \\
\hat{O}_{N L}=i \gamma\left[|E|^{2}+\frac{2 i}{\omega_{0} E} \frac{\partial}{\partial t}\left(|E|^{2} E\right)-\tau_{R} \frac{\partial|E|^{2}}{\partial t}\right]
\end{gathered}
$$

En general, como ya hemos visto en el Capítulo 2 la no-linealidad y la dispersión actúan de forma conjunta. El SSM permite obtener una solución aproximada asumiendo que en la propagación de un campo eléctrico sobre una distancia $h$, los efectos de la dispersión y la nolinealidad se pueden calcular de forma independiente. Más específicamente, la propagación desde cualquier longitud $z$ hasta otra $z+h$ se realiza en dos pasos. En el primer paso, la no-linealidad actúa sola, mientras que $\hat{O}_{D}=0$. En el segundo paso, la dispersión es calculada suponiendo que $\hat{O}_{N L}=0$.

Para estimar el error del SSM, debemos expresar el campo eléctrico propagado una distancia $h$ y asumir que $\hat{O}_{N L}$ es independiente de $z$. De esta manera, el campo eléctrico propagado resulta

$$
E(z+h, t)=e^{h\left(\hat{O}_{D}+\hat{O}_{N L}\right)} E(z, t)
$$

Sin embargo, el cálculo de la propagación por SSM realiza la siguiente aproximación $e^{h\left(\hat{O}_{D}+\hat{O}_{N L}\right)} \approx e^{h \hat{O}_{D}} e^{h \hat{O}_{N L}}$, ignorando la naturaleza no conmutativa de los operadores lineal y no-lineal [16]. Por lo tanto, empleando la ecuación de Baker-Hausdorff [137] se puede encontrar que el término del error dominante se obtiene mediante el conmutador simple $h^{2}\left[\hat{O}_{D}, \hat{O}_{N L}\right] / 2=h^{2}\left(\hat{O}_{D} \hat{O}_{N L}-\hat{O}_{N L} \hat{O}_{D}\right) / 2$, por lo que el método logra una precisión de segundo orden en un paso de tamaño $h$. Este error puede ser mejorado adoptando un procedimiento diferente, el cual calcula el efecto de la no-linealidad de forma concentrada en la mitad del paso de tamaño $h$, como se muestra en la Fig. 6.1.

La implementación del SSM es relativamente sencilla de la forma descrita en la Fig. 6.1. La FO es dividida en un número grande de segmentos que pueden no ser igualmente espaciados, 


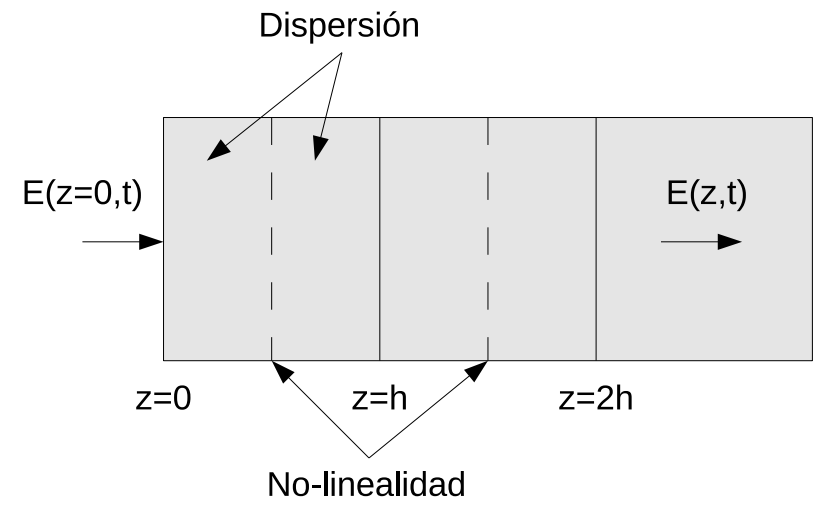

Figura 6.1: Esquema de calculo por SSM. La longitud de FO es dividida en un numero grande de segmentos de ancho $h$. Dentro de estos segmentos, el efecto de la no-linealidad es incluida en la mitad del plano, como se muestra con la linea de trazos.

por ejemplo, siguiendo alguno de los criterios formados en [52]. Un pulso óptico de entrada es propagado de un segmento a otro empleando la siguiente descripción aproximada

$$
E(z+h, t) \approx \exp \left(\frac{h}{2} \hat{O}_{D}\right)\left[\int_{z}^{z+h} \hat{O}_{N L}(\zeta) d \zeta\right] \exp \left(\frac{h}{2} \hat{O}_{D}\right) E(z, t)
$$

donde una aproximación trapezoidal de dicha integral permite estimar el error cometido. Más específicamente, el campo eléctrico $E(z, t)$ es propagado una distancia $h / 2$ sólo aplicando la dispersión mediante el algoritmo de la FFT. En la mitad del segmento, ubicada en $z+h / 2$ el campo el multiplicado por el término no-lineal, el cual representa el efecto de $\hat{O}_{N L}$ sobre todo el segmento de tamaño $h$. Finalmente, el campo es propagado sobre la una longitud restante de tamaño $h / 2$ para obtener $E(z+h, t)$.

\section{Apéndice B: Método de la matriz de transferencia TMM}

El TMM se basa en los principios de la teoría de modos acoplados y permite modelar diversos dispositivos fotónicos [56]. Los campos eléctricos de entrada y salida de una capa de material, cuyas propiedades son invariantes sobre una longitud especifica, es representada de forma unívoca por una matriz cuadrada. Los elementos de dicha matriz están directa- 
mente relacionados a las propiedades ópticas de cada capa de material. La respuesta total de un dispositivo, formado de múltiples capas, puede ser evaluada fácilmente a través del producto de un número finito de matrices acopladas entre sí.

Las características de los dispositivos fotónicos de dos puertos pueden ser modeladas mediante una matriz cuadrada $U$, donde $E_{i k}$ y $E_{o k}$ son los campos eléctricos de entrada y salida, respectivamente, y donde $k=1,2$. representa cada uno de los puertos del dispositivo. Los elementos de la matriz cuadrada son denominados $T_{m n}$ con $m$ y $n$ enteros, de tal forma que

$$
\left[\begin{array}{l}
E_{i 1} \\
E_{o 1}
\end{array}\right]=\left[\begin{array}{ll}
T_{11} & T_{12} \\
T_{21} & T_{22}
\end{array}\right]\left[\begin{array}{l}
E_{i 2} \\
E_{o 2}
\end{array}\right]
$$

Los elementos $T_{m n}$ dependen de los parámetros constructivos del dispositivo analizado. Por otra parte, la matriz $T$ puede ser transformada fácilmente en una matriz $S$, la cual permite expresar los elementos en función de los parámetros de scattering [56]. Esto es muy útil en la determinación de las pérdidas de inserción y las pérdidas de retorno de diversos dispositivos, definidos como una función de la longitud de onda, o bien de la frecuencia. Este método es robusto y flexible en el sentido que permite caracterizar el comportamiento de una amplia gama de dispositivos fotónicos, tales como: FBGs de distinto tipo, estructuras del tipo Fabry-Perot, DFBs y espejos, entre muchos otros [10,56]. Para modelar correctamente dichos dispositivos por medio del TMM se emplea una técnica que divide al dispositivo de FO de largo $L$ en $N$ secciones o tramos de tamaño $\delta l=L / N$. El campo de salida de una sección es el campo de entrada de la sección siguiente. Si una sección de longitud $\delta l_{1}$ tiene asociada a una matriz $T^{(j=1)}$, la salida de dicha matriz se usará como entrada de una segunda matriz $T^{(j=2)}$ que representa una sección contigua de largo $\delta l_{2}$. El procedimiento se extiende hasta completar el cálculo para un dispositivo de largo $L$, obteniendo una matriz de transferencia total expresada como

$$
\left[\begin{array}{c}
E_{i 1} \\
E_{o 1}
\end{array}\right]=\left[T^{(1)}\right]\left[T^{(2)}\right]\left[T^{(3)}\right] \ldots\left[T^{(N)}\right]\left[\begin{array}{l}
E_{i 2} \\
E_{o 2}
\end{array}\right]
$$


La longitud de cada sección puede diferir una de otra, como también podrá ser distinto el espaciado $\Lambda$ de la modulación del índice de refracción y su máxima variación $\Delta n$. Es importante elegir adecuadamente la longitud de cada sección, de tal forma que sus propiedades permanezcan invariantes (período, variación del índice de refracción, etc.). En caso contrario, una matriz asociada a una sección cuyos parámetros cambian, no representaría correctamente la transferencia del dispositivo. Con esta idea se asocian matrices de transferencia por cada sección suponiendo que su longitud abarca al menos un período de grabado, o sea $\delta l_{j} \gg N \Lambda_{j}$ (donde $\Lambda_{j}$ es el período de una sección $j=1 \ldots N$ ). Por otra parte, debemos notar que el modelado por medio de la matriz de scattering no puede ser aplicado como describe la Ec. 6.7 debido a que el producto de las matrices correspondientes a cada sección no resulta en la matriz final $S$.

\section{TMM aplicado al calculo de FBGs}

Como ya hemos mencionado, el TMM es fácilmente implementado mediante el calculo de las matrices de transferencia de cada una de las capas de material, definidas en termino de secciones con propiedades invariantes. Una FBG uniforme es definida mediante la teoría de modos acoplados como se describe en [10]. Los elementos de cada una de las matrices asociadas al TMM son expresadas como

$$
\begin{gathered}
T_{11}^{(j)}=\cosh \left(q \delta l_{j}\right)-\frac{i \delta \sinh \left(q \delta l_{j}\right)}{q} \\
T_{22}^{(j)}=\cosh \left(q \delta l_{j}\right)+\frac{i \delta \sinh \left(q \delta l_{j}\right)}{q} \\
T_{12}^{(j)}=-\frac{i \kappa \sinh \left(q \delta l_{j}\right)}{q} \\
T_{21}^{(j)}=\frac{i \kappa \sinh \left(q \delta l_{j}\right)}{q}
\end{gathered}
$$

donde $q=\sqrt{\delta^{2}-\kappa^{2}}, \kappa=\pi n_{\text {eff }} v / \lambda$ es la constante de acoplamiento de los modos contrapropagados dentro de la FBG $(v$ es el coeficiente de modulación del índice de refracción, también denominada visibilidad) y $\delta=2 \pi n_{e f f}\left(\lambda^{-1}-\lambda_{\text {Bragg }}^{-1}\right)$ representa el desajuste de 
fase entre los modos, en función de la diferencia entre la longitud de onda de análisis $\lambda$ y la longitud de onda de Bragg $\lambda_{\text {Bragg }}$.

Los coeficientes de reflexión $\rho$ y/o transmisión $\tau$ que se definen para una matriz $T$ deben ser calculados empleando la Ec. 6.6, anulando uno de los campos. Para calcular el coeficiente de transmisión se debe cumplir que $E_{i 1}=1$ y $E_{o 2}=0$ en dicha ecuación, resultando que se puede definir como $\tau=E_{i 2} / E_{i 1}=1 / T_{11}$. De forma análoga se puede calcular el coeficiente de reflexión como $\rho=E_{o 1} / E_{i 1}=T_{21} / T_{11}$.

El TMM requiere que se cumplan ciertas condiciones para funcionar correctamente. Primero, como los parámetros de la FBG son una función de la longitud de propagación z, la mínima longitud de una sección debe ser $\delta l_{j} \geq \Lambda_{j} K$, donde $\mathrm{K}$ es un número entero. Este valor depende de la variación del espaciado $\Lambda$ relacionado a una FBGs con chirp y el mismo determina el límite superior del número de secciones adecuado para no introducir errores sistemáticos en el cálculo. Segundo, se debe asegurar que cada sección $j$ tenga un número entero de períodos para tener transiciones suaves entre las secciones. Un cambio abrupto en la modulación del índice de refracción de la FBG equivale a tener un defecto, o sea, una discontinuidad de la fase del coeficiente de reflexión y/o transmisión que genera un pico (muchas veces indeseado) en el espectro de reflexión y/o absorción. Tercero, si hay diferencias entre las constantes de acoplamiento $\left(\kappa_{j}\right)$ entre secciones adyacentes se forman super-estructuras, formadas por otros picos en las características espectrales de una FBG. De esta forma, existe un número mínimo de secciones que permite reducir la distorsión en el cálculo de dichos dispositivos. Finalmente, al simular FBGs de período largo (LPFBG) y con mucho chirp es necesario definir una buena resolución espectral para calcular adecuadamente el retardo de grupo. 


\section{Referencias}

[1] N. A. Russo and R. Duchowicz, "High frequency pulse trains from a self-starting additive pulse mode-locked all-fiber laser," Optics Communications, vol. 283, pp. 113$117,2010$.

[2] E. Paulucci, N. A. Russo, E. Sicre, and R. Duchowicz, "Numerical and experimental comparison of an all-fiber APM laser with two-coupled linear cavities," Optics and Laser Technology, vol. 48, pp. 495-502, 2013.

[3] J. Chesnoy, Undersea fiber communication systems. Academic Press, 2002.

[4] G. Agrawal, Fiber-optic communication systems. 605 Third Avenue. New York. NY 10158-0012.: Wiley-Interscience, 2002.

[5] G. Agrawal, Lightwave technology telecommunication systems. 605 Third Avenue. New York. NY 10158-0012.: Wiley-Interscience, 2005.

[6] G. Keiser, Optical fiber communications. 1221 Ave. of the Americas. New York. NY 10020-1095.: McGraw-Hill, 2000.

[7] J. H. Winters, R. D. Gitlin, and S. Kasturia, "Reducing the effects of transmission impairments in digital fiber optic systems," IEEE Communication Magazine, vol. 6, pp. 68-76, 1993.

[8] O. E. Agazzi, M. R. Hueda, H. S. Carrer, and D. E. Crivelli, "Maximum-likelihood sequence estimation in dispersive optical channels," Journal of Lightwave Technology, vol. 23, no. 2, pp. 749-763, 2005. 
[9] M. R. Hueda, D. E. Crivelli, H. S. Carrer, and O. E. Agazzi, "Parametric estimation of IM/DD optical channels using new closed-form approximations of the signal PDF," Journal Of Lightwave Technology, vol. 25, pp. 957-975, 2007.

[10] R. Kashyap, Fiber Bragg gratings. 525 B Street, Suite 1900, San Diego, CA 921014495, USA.: Academic Press, 1999.

[11] M. Saruwatari, "All-optical signal processing for terabit/second optical transmission," Selected Topics in Quantum Electronics, vol. 6, pp. 1363-1374, 2000.

[12] J. Capmany, B. Ortega, D. Pastor, and S. Sales, "Discrete-time optical processing of microwave signals," Journal of Lightwave Technology, vol. 23, no. 2, pp. 702-723, 2005.

[13] Y. Dai, X. Chen, J. Sun, Y. Yao, and S. Xie, "Dispersion compensation based on sampled fiber Bragg gratings fabricated with reconstruction equivalent-chirp method," IEEE Photonics Technology Letters, vol. 18, no. 8, pp. 941-943, 2006.

[14] E. Desurvire, Erbium-doped fiber amplifiers. 605 Third Avenue, New York, NY 101580012: John Wiley \& Sons, 1994.

[15] M. Digonnet, Rare-earth-doped fiber lasers and amplifiers. New York. Basel: Marcel Dekker, 1993.

[16] G. Agrawal, Nonlinear fiber optics. 525 B Street, Suite 1900, San Diego, California 92101-4495: Academic Press, 1995.

[17] E. Yoshida and M. Nakazawa, "80-200 Ghz erbium doped fiber laser using a rotational harmonic mode-locking technique," Electronics Letters, vol. 32, pp. 1370-1372, 1996.

[18] M. Jiang, K. Ahn, X. Cao, P. Dasika, Y. Liang, M. Islam, A. Evans, R. Hawk, D. Nolan, and D. Weidman, "Synchronization of passively mode-locked erbium-doped fiber lasers and its application to optical communication networks," Journal of Lightwave Technology, vol. 15, pp. 2020-2028, 1997. 
[19] Y. Je and S. T. Cundiff, Femtosecond optical frequency comb: principle, operation and applications. Springer Science Business Media, Inc. Boston: Springer, 2005.

[20] M. Ablowitz, T. Horikis, and S. Nixon, "Soliton strings and interactions in modelocked lasers," Optics Communications, vol. 282, pp. 4127-4135, 2009.

[21] P. Laporta, S. Longhi, M. Marchesi, S. Taccheo, and . Svelto, "2.5 ghz and 5 ghz harmonic mode-locking of a diode-pumped bulk erbium-ytterbium glass laser at 1.5 microns," Photonics Technology Letters, vol. 7, pp. 155-157, 1995.

[22] D. W. Huang, G. C. Lin, and C. C. Yang, "Fiber Bragg based self matched additive pulse mode locked fiber lasers," Quantum Electronics, vol. 35, pp. 138-146, 1999.

[23] C. K. Nielsen, T. V. Andersen, and S. R. Keiding, "Stability analisys of an all fiber coupled cavity Fabry Perot additive pulse mode locked laser," Journal of Quantum Electronics, vol. 41, pp. 198-204, 2005.

[24] J. Williams, K. Sugden, L. Zhang, I. Bennion, and N. Doran, "In-fiber grating systems for pulse compression and complete dispersion compensation," in Optical Fibre Gratings and Their Applications, IEE Colloquium on, pp. 9/1-9/6, Jan 1995.

[25] T. Inoue, H. Tobioka, K. Igarashi, and S. Namiki, "Optical pulse compression based on stationary rescaled pulse propagation in a comblike profiled fiber," Journal of Lightwave Technology, vol. 24, no. 7, pp. 2510-2522, 2006.

[26] J. W. Nicholson, A. D. Yablon, M. F. Yan, P. Wisk, J. Fleming, E. Monberg, F. Dimarcello, R. Bise, D. J. Trevor, J. Alonzo, and T. Stockert, "The impact of nonlinearity during femtosecond pulse compression in fibers on continuum coherence," in Lasers and Electro-Optics, 2008 and 2008 Conference on Quantum Electronics and Laser Science. CLEO/QELS 2008. Conference on, pp. 1-2, 2008.

[27] N. Nishizawa and K. Takahashi, "Time-domain near-infrared spectroscopy using a wavelength-tunable narrow-linewidth source by spectral compression of ultrashort soliton pulses," Optics Letters, vol. 36, no. 19, pp. 3780-3782, 2011. 
[28] A. Hideur, T. Chartier, M. Brunel, M. Salhi, C. Ozkul, and F. Sanchez, "Mode-lock, Q-switch and CW operation of an Yb-doped double-clad fiber ring laser," Optics Communications, vol. 198, pp. 141-146, 2001.

[29] M. Nakazawa and E. Yoshida, “A 40 Ghz 850 fs regeneratively FM mode-locked polarization-maintaining erbium fiber ring laser," Photonics Technology Letters, IEEE, vol. 12, no. 12, pp. 1613-1615, 2000.

[30] M. Engelbrecht, F. Haxsen, A. Ruehl, D. Wandt, and D. Kracht, "320 fs thuliumdoped fiber-ring-laser with a pulse energy of $3.5 \mathrm{nj}$," in Conference on Lasers and Electro-Optics/Quantum Electronics and Laser Science Conference and Photonic Applications Systems Technologies, p. CFD4, OSA, 2008.

[31] H. Haus, J. Fujimoto, and E. Ippen, "Structures for additive pulse mode locking," Optic Communications, vol. 8, pp. 2068-2076, 1991.

[32] H. Haus, "Mode locking of lasers," Selected Topics in Quantum Electronics, vol. 6, pp. 1173-1185, 2000.

[33] Y.-G. Han, T. V. A. Tran, and S. B. Lee, "Wavelength-spacing tunable multiwavelength erbium-doped fiber laser based on four-wave mixing of dispersion-shifted fiber," Optics Letters, vol. 31, no. 6, pp. 697-699, 2006.

[34] J. Schröder, S. Coen, F. Vanholsbeeck, and T. Sylvestre, "Passively mode-locked Raman fiber laser with 100 Ghz repetition rate," Optics Letters, vol. 31, no. 23, pp. 3489-3491, 2006.

[35] A. B. Matsko, A. A. Savchenkov, and L. Maleki, "Normal group-velocity dispersion kerr frequency comb," Optics Letters, vol. 37, no. 1, pp. 43-45, 2012.

[36] E. D. Farnum, L. Butson, and J. N. Kutz, "Theory and simulation of dual-frequency mode-locked lasers," JOSA B, vol. 23, pp. 257-264, 2006. 
[37] K. Hagiuda, T. Hirooka, M. Nakazawa, S. Arahira, and Y. Ogawa, "40 Ghz, 100 fs stimulated-Brillouin-scattering-free pulse generation by combining a mode-locked laser diode and a dispersion-decreasing fiber," Optics Letters, vol. 30, pp. 670-672, 2005.

[38] M. Yoshida, T. Hirayama, M. Nakazawa, K. Hagimoto, and T. Ikegami, "Regeneratively mode-locked fiber laser with a repetition rate stability of $4.9 \times 10-15$ using a hydrogen maser phase-locked loop," Optics Letters, vol. 32, pp. 1827-1829, 2007.

[39] E. Paulucci, N. Russo, E. Sicre, and R. Duchowicz, "Multiplexación/demultiplexación de señales WDM mediante filtros de realimentación distribuida," in XIII Reunión de Trabajo en Procesamiento de la Información y Control, pp. 329-334, RPIC, 2009.

[40] E. Paulucci, Redes de Bragg grabadas y sus aplicaciones. Trabajo de tesis de grado. ingeniero en electrónica, Departamento de Electrotecnia. Facultad de Ingeniería. Universidad Nacional de La Plata., 2008.

[41] L. Schares, R. Paschotta, L. Occhi, and G. Guekos, "40 Ghz mode-locked fiberring laser using a Mach-Zehnder interferometer with integrated SOAs," Journal of Lightwave Technology, vol. 22, no. 3, pp. 859-873, 2004.

[42] G. Villanueva, M. Ferri, and P. Perez-Millan, "Active and passive mode-locked fiber lasers for high-speed high-resolution photonic analog-to-digital conversion," IEEE Journal of Quantum Electronics, vol. 48, no. 11, pp. 1443-1452, 2012.

[43] P. Costanzo, S. Rabal, E. Paulucci, A. Giordana, and L. B. Rossini, "Practical impairments in FBG-based true time delays," in Latin America Optics and Photonics Conference, p. LM2A.21, OSA, 2012.

[44] L. A. B. Rossini, E. Paulucci, R. Duchowicz, and E. E. Sicre, "Novel method for optical pulse characterization," Optical Engineering (enviado Mayo de 2013). 
[45] E. Paulucci, C. Lambert, A. Mantesa, and R. Duchowicz, "Análisis y caracterización de la emisión generada por procesos de "up-conversion" en fibras dopadas con yterbio," in Congreso; 92 Reunión Nacional de Física; 2007, pp. P-4, 2007.

[46] E. Paulucci, N. Russo, E. Sicre, and R. Duchowicz, "Theory and simulation of a two coupled-cavities fiber laser," in 22nd Congress of the International Commission for Optics. Light for the Development of the World, SPIE, 2011.

[47] J. Herrmann, D. Griebner, N. Zhavoronkov, A. Husakou, D. Nickel, G. Korn, J. Knight, W. Wadsworth, and P. Russel, "Experimental evidence for supercontinuum generation by fission of higher-order solitons in photonic crystal fibers," in Quantum Electronics and Laser Science Conference, 2002. QELS '02. Technical Digest. Summaries of Papers Presented at the, pp. 165-166, 2002.

[48] N. Rosanov, Y. Rozhdestvensky, V. Smirnov, and S. Federov, "Applications of BoseEinstein condensate soliton states to quantum metrology and nanotechnology," in Quantum Electronics Conference, 2003. EQEC '03. European, p. 307, 2003.

[49] D. Rickett and D. Ham, "A chip-scale electrical soliton modelocked oscillator," in Solid-State Circuits Conference, 2006. ISSCC 2006. Digest of Technical Papers. IEEE International, pp. 1716-1725, 2006.

[50] K. Koizumi, M. Yoshida, T. Hirooka, and M. Nakazawa, "10 Ghz 1.1 ps pulse generation from a harmonically and regeneratively mode-locked Yb fiber laser at 1.1 $\mu \mathrm{m}, "$ in Microopics Conference (MOC) 2011 17th, pp. 1-2, 2011.

[51] A. Agrawal and E. Holzman, "Beamformer architectures for active phased-array radar antennas," IEEE Transactions on Antennas and Propagation, vol. 47, no. 3, pp. 432442, 1999.

[52] O. V. Sinkin, R. Holzlöhner, J. Zweck, and C. R. Menyuk, "Optimization of the splitstep Fourier method in modeling optical-fiber communications systems," Journal of Lightwave Technology, vol. 21, no. 1, p. 61, 2003. 
[53] O. E. Martínez, R. L. Fork, and J. P. Gordon, "Theory of passively mode-locked lasers for the case of a nonlinear complex-propagation coefficient," JOSA B, vol. 2, pp. $753-760,1984$.

[54] O. E. Martínez, R. L. Fork, and J. P. Gordon, "Theory of passively mode-locked lasers including self-phase modulation and group-velocity dispersion," Optics Letters, vol. 9, pp. 156-158, 1984.

[55] G. Agrawal, Lightwave Technology Components and Devices. 525 B Street, Suite 1900, San Diego, California 92101-4495: Academic Press, 1994.

[56] B. E. A. Saleh and M. C. Teich, Fundamentals of Photonics. Wiley-Interscience, 2007.

[57] G. Kant, P. Patel, S. Wijnholds, M. Ruiter, and E. van der Wal, "EMBRACE: a multi-beam 20,000-element radio astronomical phased array antenna demonstrator," IEEE Transactions on Antennas and Propagation, vol. 59, no. 6, pp. 1990-2003, 2011.

[58] R. Feger, C. Pfeffer, W. Scheiblhofer, C. Schmid, M. Lang, and S. Stelzer, "A 77 Ghz cooperative radar system based on multi-channel FMCW stations for local positioning applications," IEEE Transactions on Microwave Theory and Techniques, vol. 61, no. 1, pp. 676-684, 2013.

[59] Y. Fujii, A. Gonzalez, M. Kroug, K. Kaneko, A. Miyachi, T. Yokoshima, K. Kuroiwa, H. Ogawa, K. Makise, Z. Wang, and Y. Uzawa, "The first six ALMA band 10 receivers," IEEE Transactions on Terahertz Science and Technology, vol. 3, no. 1, pp. 39-49, 2013.

[60] T. Erdogan, "Fiber grating spectra," Journal of Lightwave Technology, vol. 15, no. 8, pp. 1277-1294, 1997.

[61] K. Ennser, M. N. Zervas, and R. I. Laming, "Optimization of apodized linearly chirped fiber gratings for optical communications," Quantum Electronics, IEEE Journal of, vol. 34, no. 5, pp. 770-778, 1998. 
[62] H. J. R. Dutton, Understanding optical communications. The ITSO Networking Series, Upper Saddle River, NJ: Prentice Hall PTR, 1998.

[63] Komukai, Inui, and Nakazawa, "The design of dispersion equalizers using chirped fiber Bragg gratings," IEEE Journal of Quantum Electronics, vol. 36, no. 4, pp. 409417, 2000.

[64] S. Doucet, S. LaRochelle, and M. Morin, "Reconfigurable dispersion equalizer based on phase-apodized fiber Bragg gratings," Journal of Lightwave Technology, vol. 26, no. 16, pp. 2899-2908, 2008.

[65] G. Agrawal, Applications of nonlinear fiber optics. 525 B Street, Suite 1900, San Diego, California 92101-4495: Academic Press, 2001.

[66] K. C. Byron, K. Sugden, T. Bricheno, and I. Bennion, "Fabrication of chirped bragg gratings in photosensitive fibre," Electronics Letters, vol. 29, no. 18, pp. 1659-1660, 1993.

[67] B. Eggleton, P. A. Krug, and L. Poladian, "Dispersion compensation by using Bragggrating filters with self-induced chirp," in Conference on Optical Fiber Communication, p. ThK3, Optical Society of America, 1994.

[68] K. O. Hill, F. Bilodeau, B. Malo, T. Kitagawa, S. Theriault, D. C. Johnson, and J. Albert, "Aperiodic in-fiber Bragg gratings for optical fiber dispersion compensation," in Conference on Optical Fiber Communication, p. PD2, Optical Society of America, 1994.

[69] G. P. Agrawal and S. Radic, "Phase-shifted fiber bragg gratings and their application for wavelenght demultiplexing," IEEE Photonic Technology Letters, vol. 6, p. 8, 1994.

[70] H. Olesen, J. Salzman, B. Jonsson, and B. Tromborg, "Single-mode stability of DFB lasers with longitudinal bragg detuning," Photonics Technology Letters, IEEE, vol. 7, no. 5, pp. 461-463, 1995. 
[71] C. Theis, P. Jongwoo, P. Kiely, G. Tohmon, W. Ping, U. Chakrabarti, and J. Osenbach, "Wavelength stability of DFB lasers for non-hermetic applications," in Electronic Components and Technology Conference, 2001. Proceedings., 51st, pp. 632-636, 2001.

[72] H. Nasu, I. Mukaihara, T. Takagi, M. Oike, T. Nomura, and A. Kasukawa, "25 Ghz spacing wavelength-monitor integrated DFB laser module for DWDM applications," IEEE Photonics Technology Letters, vol. 15, no. 2, pp. 293-295, 2003.

[73] S. Legoubin, M. Douay, P. Bernage, P. Niay, S. Boj, and E. Delevaque, "Free spectral range variations of grating-based Fabry-Perot filters photowritten in optical fibers," JOSA A, vol. 12, p. 8, 1994.

[74] J. Dakin and B. Culshaw, Optical fiber sensors: principles and components. Artech House: University of Michigan, 1996.

[75] M. Born and E. Wolf, Principles of optics: electromagnetic theory of propagation, interference and diffraction of light. Cambridge University Press, 1999.

[76] R. Pfleegor and L. Mandel, "Interference of independent photon beams," Physical Review, vol. 159, pp. 1084-1088, 1967.

[77] P. Costanzo, L. B. Rossini, C. Lambert, E. Paulucci, R. Duchowicz, and E. Sicre, "Diseño y simulación de una red óptica pasiva para servicios de datos, voz y video," in XIII Reunión de Trabajo en Procesamiento de la Información y Control, pp. 907-912, RPIC, 2012.

[78] Hibino, Kitagawa, Hill, Bilodeau, Malo, Albert, and Johnson, "Wavelength division multiplexer with photoinduced Bragg gratings fabricated in a planar-lightwavecircuit-type asymmetric Mach-Zehnder interferometer on Si," IEEE Photonics Technology Letters, vol. 8, no. 1, pp. 84-86, 1996. 
[79] G. Nykolak, M. R. X. de Barros, T. N. Nielsen, and L. Eskildsen, "All-fiber active adddrop wavelength router," IEEE Photonics Technology Letters, vol. 9, no. 5, pp. 605606, 1997.

[80] R. Kop and R. Sprik, "Phase-sensitive interferometry with ultrashort optical pulses," Review of Scientific Instruments, vol. 66, no. 12, pp. 5459-5463, 1995.

[81] T. Okada, K. Komori, K. Goshima, S. Yamauchi, I. Morohashi, T. Sugaya, M. Ogura, and N. Tsurumachi, "Development of high resolution Michelson interferometer for stable phase-locked ultrashort pulse pair generation," Review of Scientific Instruments, vol. 79, no. 10, pp. 103101.1-7, 2008.

[82] K. Hattori, M. Abe, J. Albert, A. Bilodeau, K. Hill, Y. Hibino, T. Kitagawa, and K. Oguchi, "Coherent crosstalk of an optical add/drop filter with Bragg gratings in a PLC Mach-Zehnder interferometer for optical LAN," IEEE Photonics Technology Letters, vol. 11, no. 2, pp. 272-274, 1999.

[83] T. Hashimot, Y. Nasu, Y. Sakamaki, K. Hattori, Y. Inoue, H. Takahashi, H. Kawakami, and E. Yoshida, "Compact dqpsk demodulator with interwoven double MachZehnder interferometer using planar lightwave circuit," in 34th European Conference on Optical Communication, 2008. ECOC 2008., pp. 1-2, 2008.

[84] E. Greer, Y. Kimura, K. Suzuki, E. Yoshida, and M. Nakazawa, "Generation of 1.2 ps, 10 ghz pulse train from all-optically modelocked, erbium fibre ring laser with active nonlinear polarisation rotation," IEEE Electronics Letters, vol. 30, no. 21, pp. 1764-1765, 1994.

[85] Y. Kim, A. Simard, P. Chretien, and S. LaRochelle, "Millimeter-wave synthesizer based on spectral filtering of a phase-modulated laser using a chirped FBG with tunable distributed phase-shifts," Lightwave Technology, Journal of, vol. 27, no. 22, pp. 5183-5191, 2009. 
[86] M. Li, C. Wang, W. Li, and J. Yao, "An unbalanced temporal pulse-shaping system for chirped microwave waveform generation," IEEE Transactions on Microwave Theory and Techniques, vol. 58, no. 11, pp. 2968-2975, 2010.

[87] T. Pfeiffer, "Mode-locked fiber ring laser," 1996. United States Patent. Patent Number 5546414 .

[88] J. Vasseur, M. Hanna, J. Dudley, J. P. Goedgebuer, J. Yu, G. K. Chang, and J. R. Barry, "Alternate multiwavelength picosecond pulse generation by use of an unbalanced mach-zehnder interferometer in a mode-locked fiber ring laser," IEEE Journal of Quantum Electronics, vol. 43, no. 1, pp. 85-96, 2007.

[89] D. Mechin, P. Yvernault, L. Brilland, and D. Pureur, "Influence of Bragg gratings phase mismatch in a Mach-Zehnder-based add-drop multiplexer," Journal of Lightwave Technology, vol. 21, no. 5, p. 1411, 2003.

[90] J. Kim, G. Li, and K. Winick, "Design and fabrication of a glass waveguide optical add-drop multiplexer by use of an amorphous-silicon overlay distributed Bragg reflector," Applied Optics, vol. 43, no. 3, pp. 671-677, 2004.

[91] J. Segarra, V. Sales, and J. Prat, "Agile reconfigurable and traffic adapted all-optical access-metro networks," in 11th International Conference on Transparent Optical Networks ICTON 2009, pp. 1-6, 2009.

[92] P. Iovanna, F. Testa, R. Sabella, A. Bianchi, M. Puleri, M. R. Casanova, and A. Germoni, "Packet-optical integration nodes for next generation transport networks," IEEE/OSA Journal of Optical Communications and Networking, vol. 4, no. 10, pp. 821-835, 2012.

[93] R. Mailloux, Phase array antenna handbook. 685 Canton Street, Norwood, MA 02062: Artech House Inc., 2005.

[94] R. Mailloux, "Phased array theory and technology," Proceedings of the IEEE, vol. 70, no. 3, pp. 246-291, 1982. 
[95] K. Warnick, B. Jeffs, J. Landon, J. Waldron, D. Jones, J. Fisher, and R. Norrod, "Phased array antenna design and characterization for next-generation radio telescopes," in IEEE International Workshop on Antenna Technology, 2009. iWAT 2009., pp. 1-4, 2009.

[96] E. Lier and R. Melcher, "A modular and lightweight multibeam active phased receiving array for satellite applications: design and ground testing," IEEE Antennas and Propagation Magazine, vol. 51, no. 1, pp. 80-90, 2009.

[97] M. Fakharzadeh, Optical and microwave beamforming for phased array antennas. Phd in electrical and computer engineering, Intelligent Integrated Photonics and Radio Laboratory. University of Waterloo. Ontario. Canada., 2008.

[98] M. Fakharzadeh, P. Mousavi, S. Safavi-Naeini, and S. Jamali, "The effects of imbalanced phase shifters loss on phased array gain," IEEE Antennas and Wireless Propagation Letters, vol. 7, pp. 192-196, 2008.

[99] I. Frigyes and A. Seeds, "Optically generated true-time delay in phased-array antennas," IEEE Transactions on Microwave Theory and Techniques, vol. 43, no. 9, pp. 2378-2386, 1995.

[100] H. Rideout, J. Seregelyi, and J. Yao, "A true time delay beamforming system incorporating a wavelength tunable optical phase-lock loop," IEEE Journal of Lightwave Technology, vol. 25, no. 7, pp. 1761-1770, 2007.

[101] B.-M. Jung and J. Yao, "A two-dimensional optical true time-delay beamformer consisting of a fiber bragg grating prism and switch-based fiber-optic delay lines," IEEE Photonics Technology Letters, vol. 21, no. 10, pp. 627-629, 2009.

[102] R. Ziemer and W. Tranter, Principles of communications. 605 Third Avenue, New York, NY 10158-0012: John Wiley \& Sons, 2008.

[103] D. P. Abellán, J. C. Francoy, and F. R. Pascual, Sistema de comunicaciones ópticas. Universidad Politécnica de Valencia, 2007. 
[104] L. A. Bulus-Rossini, P. A. Costanzo-Caso, R. Duchowicz, and E. E. Sicre, "RadonWigner transform processing for optical communication signals," SPIE Proceeding Conference. Optics and Photonics for Information Proceesing IV, 77971D., pp. 77971D-77971D-10, 2010.

[105] C. Dorrer, "Characterization of nonlinear phase shifts by use of the temporal transport-of-intensity equation," Optics Letters, vol. 30, no. 23, pp. 3237-3239, 2005.

[106] L. A. Bulus-Rossini, P. A. Costanzo-Caso, R. Duchowicz, and E. E. Sicre, "Pulse characterization system for optical communication signals," Optical Engineering (enviado Mayo de 2013).

[107] F. Lewis, "Frequency and time standards," Proceedings of the IRE, vol. 43, no. 9, pp. 1046-1068, 1955.

[108] J. Helmer, "Maser oscillators," Journal of Applied Physics, vol. 28, no. 2, pp. 212-215, 1957.

[109] W. Smith, "Microwave amplification by MASER techniques," IBM Journal of Research and Development, vol. 1, no. 3, pp. 232-238, 1957.

[110] G. Dacey, "Optical MASERs," in IEEE International Solid-State Circuits Conference 1961. Digest of Technical Papers., vol. IV, pp. 96-97, 1961.

[111] E. Snitzer, "Proposed fiber cavities for optical MASERs," Journal of Applied Physics, vol. 32, no. 1, pp. 36-39, 1961.

[112] W. E. Lamb, "Theory of an optical MASER," Phys. Rev., vol. 134, pp. A1429-A1450, 1964.

[113] A. Siegman, Lasers. Mill Valley, CA: University Science Books, 1986.

[114] N. Hodgson and H. Weber, Laser resonators and beam propagation. Fundamentals, advanced concepts and applications. Springer. Springer Series in Optical Sciences, 2005. 
[115] R. Kashyap, J. Armitage, R. Wyatt, S. Davey, and D. Williams, "All-fibre narrowband reflection gratings at 1500 nm," Electronics Letters, vol. 26, pp. 730-732, may 1990.

[116] G. Ball, W. Morey, and W. Glenn, "Standing-wave monomode erbium fiber laser," Photonics Technology Letters, IEEE, vol. 3, no. 7, pp. 613-615, 1991.

[117] G. Ball, W. Glenn, W. Morey, and P. Cheo, "Modeling of short, single-frequency, fiber lasers in high-gain fiber," Photonics Technology Letters, IEEE, vol. 5, no. 6, pp. 649-651, 1993.

[118] G. Ball and W. Glenn, "Design of a single-mode linear-cavity erbium fiber laser utilizing bragg reflectors," Lightwave Technology, Journal of, vol. 10, no. 10, pp. 13381343, 1992.

[119] Y. T. Chieng and R. Minasian, "Tunable erbium-doped fiber laser with a reflection Mach-Zehnder interferometer," IEEE Photonics Technology Letters, vol. 6, no. 2, pp. 153-156, 1994.

[120] S. Namiki, C. Yu, and H. Haus, "Observation of nearly quantum-limited timing jitter in an all-fiber ring laser," JOSA B, vol. 13, pp. 2817-2823, 1996.

[121] C. Giles, C. Burrus, D. DiGiovanni, N. Dutta, and G. Raybon, "Characterization of erbium-doped fibers and application to modeling $980 \mathrm{~nm}$ and $1480 \mathrm{~nm}$ pumped amplifiers," Photonics Technology Letters, IEEE, vol. 3, no. 4, pp. 363-365, 1991.

[122] C. Ye, M. Hempstead, D. Hewak, and D. Payne, "Proposal for an $\operatorname{Er}^{3+} /$-doped chalcogenide glass fiber upconversion laser operating at $980 \mathrm{~nm}$ and pumped at 1480 nm," Photonics Technology Letters, IEEE, vol. 9, no. 8, pp. 1104-1106, 1997.

[123] V. Zotov, M. Likhachev, A. Tomashuk, A. Kosolapov, M. Bubnov, M. Yashkov, A. Guryanov, and E. Dianov, "Radiation resistant Er-doped fibers: optimization of pump wavelength," IEEE Photonics Technology Letters, vol. 20, no. 17, pp. 1476$1478,2008$. 
[124] M. Dinand and C. Schutte, "Theoretical modeling of relaxation oscillations in erdoped waveguide lasers," Journal of Lightwave Technology, vol. 13, no. 1, pp. 14-23, 1995.

[125] R. Rangel-Rojo and M. Mohebi, "Study of the onset of self-pulsing behaviour in an Er-doped fibre laser," Optics Communications, vol. 137, no. 1?3, pp. 98-102, 1997.

[126] D. D. Hudson, K. W. Holman, R. J. Jones, S. T. Cundiff, J. Ye, and D. J. Jones, "Mode-locked fiber laser frequency-controlled with an intracavity electro-optic modulator," Optics Letters, vol. 30, no. 21, pp. 2948-2950, 2005.

[127] D. D. Hudson, Mode-locked fiber lasers: development and application. Ph.d. dissertation, Department of Physics, University of Colorado, 2009.

[128] N. Russo and R. Duchowicz, "High frequency fiber laser emission generated by pump spiking," Optics Communications, vol. 281, no. 13, pp. 3532-3537, 2008.

[129] L. Ljung, System identification: theory for the user. Prentice Hall, 1993.

[130] M. Vidyasagar, Nonlinear systems analysis. Prentice Hall, 2002.

[131] B. Porat, A course in digital signal processing. 605 Third Avenue. New York. NY 10158-0012.: John Wiley \& Sons, Inc., 1997.

[132] S. H. Strogatz, Nonlinear dynamics and chaos: with applications to physics, biology, chemistry, and engineering. Perseus Books, 1994.

[133] P. Glas, M. Naumann, I. Reng, L. Dong, and J. Caplen, "High repetition rate fiber laser coupled to a linear cavity generating picosecond pulses," Fiber and Integrated Optics, vol. 17, pp. 207-212, 1997.

[134] J. Lee and D. Kim, "Spectrum-sliced Fourier-domain low-coherence interferometry for measuring the chromatic dispersion of an optical fiber," Applied Optics, vol. 46, no. 29, pp. 7289-7296, 2007. 
[135] F. Cakoni and D. Colton, Qualitative methods in inverse scattering theory. Springer, 2006.

[136] R. A. Fisher and W. Bischel, "The role of linear dispersion in plane-wave self-phase modulation," Applied Physics Letters, vol. 23, pp. 661-663, 1973.

[137] R. Scharf, "The Campbell-Baker-Hausdorff expansion for classical and quantum kicked dynamics," Journal of Physics A: Mathematical and General, vol. 21, no. 9, p. 2007, 1988 . 